\title{
DESIGN OF A LINEAR LC DIGITALLY CONTROLLED OSCILLATOR USING TOPOGRAPHICAL FIELD MAPS
}

by

SHAKEEB ABDULLAH

A thesis submitted to the Faculty of Graduate and Postdoctoral Affairs in partial fulfillment of the requirements for the degree of

\section{Master of Applied Science}

in

\section{Electrical Engineering}

Ottawa-Carleton Institute for Electrical and Computer Engineering Department of Electrical and Computer Engineering

Carleton University

Ottawa, Ontario

(C) Copyright

Shakeeb Abdullah, 2018 
Copyright (C) 2018. No parts of this document or the material within it may be re-produced, transcribed, copied, reverse engineered, duplicated or be used by any means or method without the prior permission of the author and those who holds the rights to them except for that which has been prescribed under the clause regarding thesis reproduction embedded inside (and that which is in conjunction with) the acts and laws of Carleton University Institution and of Canada regarding the distribution of graduate work. 


\begin{abstract}
The purpose of this thesis was to present a new perspective on how to design extremely linear digitally controlled oscillators. This was achieved by introducing a new concept known as linearity field maps. The linearity response of the measured DCO had an $\mathrm{R}^{2}$ value of 0.996; which was extremely linear. The DCO employed equal bank steps for all bank sets to achieve this linearity. The measured frequency response of the DCO was from $6.66 \mathrm{GHz}$ to $5.45 \mathrm{GHz}$. The DCO had fine tuning steps of $20.5 \mathrm{kHz}$ resolution and a tuning range of $20 \%$. The measured FoM was between $-175 \mathrm{dBc} / \mathrm{Hz}$ and $-181 \mathrm{~dB} / \mathrm{Hz}$ (throughout the entire frequency span of the DCO and for various power levels). The thesis experiment was a success in showing how to design a linear DCO, the DCO in the thesis worked even though the frequency was shifted from simulated results, the point of the thesis was made. The theorem and perspective introduced in this thesis held true.
\end{abstract}




\section{EXECUTIVE SUMMARY}

The reason for this report was to satisfy the requirements for MASc graduate degree at Carleton University and the purpose of this report was to present a novel outlook on how to design an extremely linear digitally controlled oscillator. This was achieved by introducing a new concept known as linearity field maps. The simulated and theoretical frequency response of the $\mathrm{DCO}$ was from $7.1 \mathrm{GHz}$ to $5.7 \mathrm{GHz}$ whereas the measured frequency response of the DCO was from 6.6 Ghz to $5.5 \mathrm{GHz}$. This caused all the linear coefficients of the measured DCO to be mismatched with the initial theoretical values. However when a new theoretical DCO that ranges with the same frequency span as the measured DCO was compared, the coefficient errors were less than $1 \%$, proving that the design technique presented in this thesis was valid. The $\mathrm{R}^{2}$ value of the measured DCO was 0.996 which was extremely linear based on the requirements of the author (less than $0.5 \%$ error from perfect linearity).

The theoretical phase noise and simulated phase noise concurred with each other $(-125 \mathrm{dBc} / \mathrm{Hz}$ at carrier frequency $6.5 \mathrm{GHz}$ and $1 \mathrm{MHz}$ offset) however the measured phase noise was almost $10 \mathrm{~dB}$ worse. About $2-3 \mathrm{~dB}$ loss could have been attributed due to poor CML performance shown through simulations. Some digital noise bleeding could have also played a role in reducing the oscillators performance as was experimentally observed, nevertheless FoM was still reasonable and respectable with FoM better than or equal to $-175 \mathrm{dBc} / \mathrm{Hz}$ throughout the entire span of the DCO (at it's highest frequencies when all banks are off and at its lowest frequency when all banks are on).

The measured Bank set 1 had a frequency step size of $66.4 \mathrm{MHz}$. The measured Bank set 2 upper limit frequency step size was $9.91 \mathrm{MHz}$ and lower limit frequency step size was $6.20 \mathrm{MHz}$. The measured Bank set 3 had upper limit frequency step size of $2.09 \mathrm{MHz}$ and lower limit frequency step size $1.29 \mathrm{MHz}$. The measured Bank set 4 had upper limit frequency step size of $469 \mathrm{kHz}$ and lower limit frequency step size of $267 \mathrm{kHz}$. Bank set 5 to 11 did not behave as intended. Further investigation needs to be done as to why these bank sets did not function properly; however it should be noted that the DCO was purposely designed with successively smaller and smaller bank sets until the frequency steps would fail - to determine the minimum practical resolution of the DCO due to technological and parasitic limitations. The frequency settling time of the DCO also took too long; however it is believed that it can be easily rectified by using bigger buffer drivers between the digital control signals and the banks of the DCO. Nevertheless, the overall thesis experiment was 
a success in showing how to design a linear digitally controlled oscillator, the DCO in the thesis worked even though the measured frequency was shifted, and the point of the thesis was made. 


\section{ACKNOWLEDGEMENTS}

I would like to start of this section with a quote in Islam that was given by the Islamic Prophet Muhammad (صلى اله عليه وسلم) from Allah (سبحانه و تعالى) or God as it is so commonly referred to in Western literature; of that which has been recorded in the Islamic Hadeeth Text "Saheeh Al-Jaami As-Sagheer \#3014" that says: "Whoever is not thankful for the small things, will not be thankful for the greater things. And whoever does not thank the people, does not thank Allah". Henceforth, as a believer in the Islamic Faith, I would like to start off by thanking all the people in my life that has shaped and helped me in producing this thesis; the ones whom I recall and ones whom I do not; from my co-workers, supervisors, team members, managers, and family members, to my peers, technicians, colleagues, faculty members, and advisers; they all played a crucial role whether it be small or big.

First and foremost, I would like to thank my very own Supervisor John W. M. Rogers for being extremely patient with me throughout the most difficult of times, and encouraging me to finish my thesis despite down trodden moments of lackadaisical ambition, interest, and despair. I could not have completed this work without his aid, support, and help. If it were not for John, I would not have the funding, skill, technical prowess, confidence, or capability to go through such a project like that which is provided throughout this thesis. He was the most crucial person from the academic perspective.

Like John, I would like to thank my Manager at Microsemi/Microchip Corporation Krste Mitric, who played the same role as John did but from the company or corporate perspective. Everything that has been applicable to John can be said about Krste, and for the sake of redundancy can be left at that. I would like to thank Kobe Situ, William Roberts, and Kristopher Kshonze for their advice during the review of the thesis project. I would like to thank Brendon Manning, Peter Leung, Faizal Warsalee, and Yasuhide Goto for aiding with layout and chip-tape out and Dale Matthie and Crystal Leung for their help in designing/soldering the PCB. I would also like to thank everyone else at Microsemi (Microchip) Corporation that allowed this project to happen.

I would like to thank Nagui Mikhail for his assistance in providing components for PCB and his fabrication advice. I would like to thank my team members Tony (Tuoxin) Wang and Juan Heredia for their support and advice, and I would like to thank Tahseen Haque for his encouragement. And lastly but most importantly I would like to thank my family members, especially my parents, for I could not have done this thesis without their support. 


\section{TABLE OF CONTENTS}

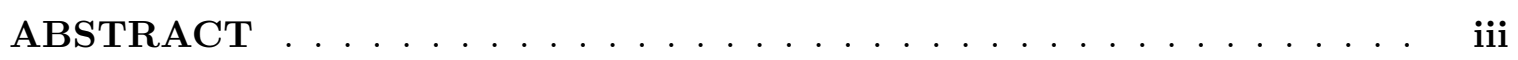

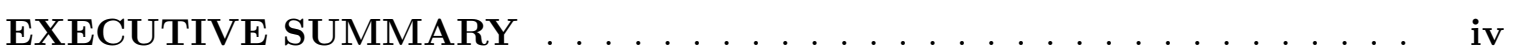

ACKNOWLEDGEMENTS . . . . . . . . . . . . . . . . vi

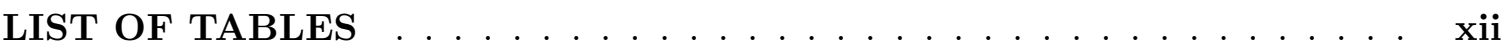

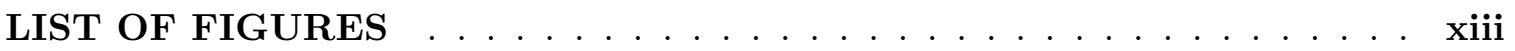

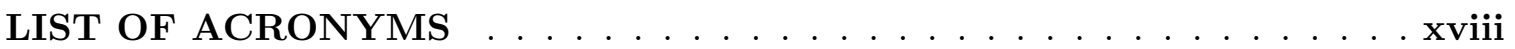

CHAPTER 1: INTRODUCTION ................ 1

1.1 Oscillators and Phase-Locked Loops . . . . . . . . . . . . . . . . . . . 1

1.1.1 Analog Phase-Locked Loops . . . . . . . . . . . . . . . . 1

1.1.2 All-Digital Phased-Locked Loop . . . . . . . . . . . . . . . . . . . 2

1.2 Motivation for Thesis Research . . . . . . . . . . . . . . . . . 3

1.3 Thesis Scope and Research Contribution . . . . . . . . . . . . . . 4

1.4 Thesis Organization and Outline . . . . . . . . . . . . . . 4

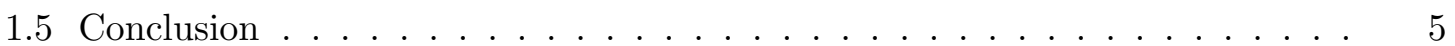

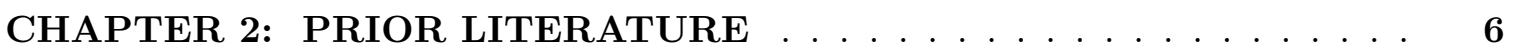

2.1 Least Mean Square Calibration Method . . . . . . . . . . . . . . . . . 6

2.2 High Linearity VCO Using Modified ICO . . . . . . . . . . . . . . 8

2.3 Highly Linear DCO in $65-n m$ CMOS . . . . . . . . . . . . . . . . 10

2.4 Other Literature in Linearizing Frequency Response of Oscillators . . . . . . 13

2.5 Conclusion . . . . . . . . . . . . . . . . . . . . . . . 14

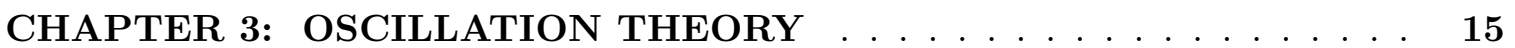

3.1 Oscillators and Barkhausen Criterion . . . . . . . . . . . . . . 15

3.2 Parallel LC oscillators . . . . . . . . . . . . . . . . . . . . . 15

3.2.1 Implementation of negative resistance . . . . . . . . . . . . . . 17

3.2 .2 Analysis of $-g_{m}$ oscillator $\ldots \ldots \ldots \ldots \ldots$

3.2 .3 Biasing of $-g_{m}$ oscillator $\ldots \ldots \ldots \ldots \ldots$

3.3 Phase noise performance of $-g_{m}$ oscillator $\ldots \ldots \ldots$. . . . . . . . 20

3.4 Figure of Merit $(\mathrm{FoM}) \ldots \ldots \ldots \ldots \ldots 22$ 
3.5 Conclusion $\ldots \ldots \ldots \ldots \ldots \ldots \ldots \ldots \ldots \ldots \ldots \ldots \ldots \ldots \ldots$

CHAPTER 4: LINEAR THEOREM \& DESIGN . . . . . . . . . . . 23

4.1 Inherent Nature of LC Oscillators . . . . . . . . . . . . . . . 23

4.2 Statistical Analysis of Linear Approximation . . . . . . . . . . . . . 24

4.3 Using $\mathrm{R}^{2}$ to Choose Region of Linearity . . . . . . . . . . . . . . . 27

4.3 .1 Topographical linearity field-maps . . . . . . . . . . . . . 27

4.4 Example of Using $\mathrm{R}^{2}$ Field Maps . . . . . . . . . . . . . . . . . 29

4.5 Implementing a Physical DCO Using $\mathrm{R}^{2}$ Linearization Technique . . . . . . 31

4.5.1 LC oscillator core . . . . . . . . . . . . . . . . . 31

4.5.2 Capacitor banks for equal frequency step sizes . . . . . . . . . . 32

4.5.3 Banks on source side of negative gm transistors . . . . . . . . . . . 34

4.6 Tuning Range . . . . . . . . . . . . . . . . . . . . . . . 35

4.6.1 Relationship between tuning range and linearity . . . . . . . . . . 35

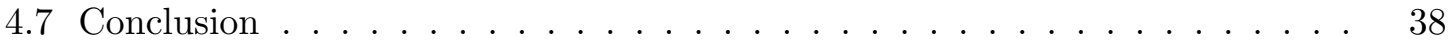

CHAPTER 5: SCHEMATIC DESIGN . . . . . . . . . . . . . 39

5.1 Schematic Design of DCO Core and DCO Core Buffers . . . . . . . . . . 39

5.2 Schematic Implementation of Digital Banks . . . . . . . . . . . . . 41

5.3 Schematic Implementation of CML Dividers . . . . . . . . . . . . . . 44

5.4 Voltage Regulator . . . . . . . . . . . . . . . . . . . 45

5.5 Digital Circuit to Control DCO . . . . . . . . . . . . . . 45

5.5.1 Binary to thermometer decoders . . . . . . . . . . . . 46

5.5.2 Serial to parallel interface $(\mathrm{S} 2 \mathrm{PI}) \ldots \ldots \ldots 4 . \ldots \ldots$

5.5 .3 Muxes . . . . . . . . . . . . . . . . . . . . . . 48

5.5 .4 Ring Oscillator . . . . . . . . . . . . . . . . 48

5.6 Conclusion . . . . . . . . . . . . . . . . . . . . . . . 49

CHAPTER 6: LAYOUT DESIGN . . . . . . . . . . . . . . 50

6.1 DCO Tank, Digital Banks, and Core Buffer Layout . . . . . . . . . . . 50

6.1 .1 Layout of Digital Banks . . . . . . . . . . . . . . . 52

6.2 CML Divider Layout $\ldots \ldots \ldots$

6.3 Layout of Buffers Connecting to the Bonding Pads . . . . . . . . . . . 55

6.4 Layout of Binary to Thermometer Decoder . . . . . . . . . . . . . . 55

6.5 Layout of 57 Bit Serial to Parallel Converter . . . . . . . . . . . . . . . 57 
6.5 .1 Layout of Shift-Register . . . . . . . . . . . . . . 58

6.6 Layout of Digital Clock and Dividers . . . . . . . . . . . . . . 58

6.7 Layout of the DCO With Digital Circuitry . . . . . . . . . . . . . 59

6.8 Layout of Second DCO . . . . . . . . . . . . . . . . . . 61

6.9 Full Chip Layout for Tape-out . . . . . . . . . . . . . . . . . . . . . 62

6.10 Conclusion . . . . . . . . . . . . . . . . . . 64

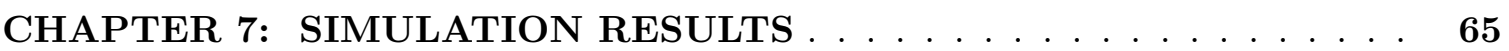

7.1 Transient Simulations of DCO . . . . . . . . . . . . . . . 65

7.2 Frequency Simulations of DCO . . . . . . . . . . . . . . 66

7.2.1 Frequency simulations of Bank set $1 \ldots \ldots 66$

7.2.2 Frequency simulations of Bank 2 . . . . . . . . . . . . . . 67

7.2.3 Frequency simulations of Bank Set $3 \ldots \ldots$. . . . . . . . . . . 67

7.2.4 Frequency simulations of Bank Set $4 \ldots \ldots$. . . . . . . 68

7.2.5 Frequency simulations of Bank Set $5 \ldots \ldots 68$

7.2.6 Frequency simulations of Bank Set $6 \ldots \ldots$. . . . . . . . 68

7.2.7 Frequency simulations of Bank set 7 to Bank set $11 \ldots$. . . . . . 69

7.2.8 Summary of frequencies \& step sizes of the Bank sets . . . . . . . . . 69

7.3 Phase Noise Simulations of DCO . . . . . . . . . . . . . . . . . . 70

7.4 Power vs Phase Noise Simulations . . . . . . . . . . . . . . . . . . . . . 72

7.4.1 Effects on PN due to CML dividers . . . . . . . . . . . . . . 73

7.4 .2 FoM . . . . . . . . . . . . . . . . . . . . 74

7.5 CML Transient Simulations _ . . . . . . . . . . . . . . . . 75

7.6 Tunning Range of Oscillator . . . . . . . . . . . . . . . . . . . . . 75

7.7 Digital Clock Simulation of Ring Oscillator . . . . . . . . . . . . 76

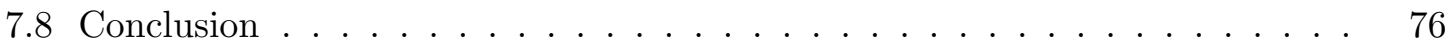

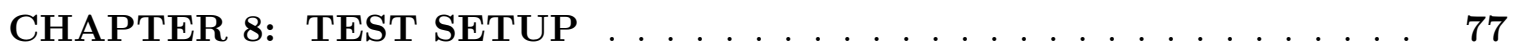

8.1 The DCO Test Chip Die . . . . . . . . . . . . . . . 77

8.2 Bonding and Packaging of Die . . . . . . . . . . . . . . . . 79

8.3 Main Test PCB to Test the DCO . . . . . . . . . . . . . . . . . . 80

8.4 Digital Test Circuit PCB . . . . . . . . . . . . . . . . . . . . 83

8.4 .1 Serial communication . . . . . . . . . . . . . . . 84

8.4.2 PCB of DCO digital interface . . . . . . . . . . . . . 85

8.5 Software to Automate Testing and Control the DCO . . . . . . . . . . 86 
8.6 Additional Test Circuit PCB . . . . . . . . . . . . . . . . . 87

8.7 Equipment Used to Test DCO . . . . . . . . . . . . . . . 88

8.8 Conclusion . . . . . . . . . . . . . . . . . . . . . 90

CHAPTER 9: EXPERIMENTAL RESULTS . . . . . . . . . . . . . . 91

9.1 Transient Results . . . . . . . . . . . . . . . . . . . . . . . . 91

9.1.1 Transient results of oscillator . . . . . . . . . . . . . . 91

9.1.2 Transient results of CML divide by $2 \ldots \ldots \ldots$

9.1.3 Transient simulation of internal ring oscillator (digital clock) . . . . 92

9.2 FFT Results . . . . . . . . . . . . . . . . . . . . . 93

9.3 Frequency Measurements . . . . . . . . . . . . . . . . . 96

9.3.1 Frequency measurements of Bank set $1 \ldots \ldots 96$

9.3.2 Frequency measurements of Bank set $2 \ldots \ldots$. . . . . . . . . . 96

9.3.3 Frequency measurements of Bank set $3 \ldots \ldots$. . . . . . . . . . . 97

9.3.4 Frequency measurements of Bank set $4 \ldots \ldots 9$

9.3.5 Frequency measurements of Bank set $5 \ldots \ldots$. . . . . . . . . . 99

9.3.6 Frequency measurements of Bank set 6 to Bank set 11 . . . . . . . 100

9.3.7 Summarized Frequency Step Measurements . . . . . . . . . . . . . . . 100

9.4 Frequency Settling Time Measurements . . . . . . . . . . . . . . . . . . 100

9.4.1 Multiple bank switch - cycling between Bank 1 and Bank 2 . . . . . . 102

9.4.2 Multiple bank switch - cycling between Bank sets 1, 2, and 3 . . . . 103

9.4.3 Multiple bank switch - cycling between Bank sets 2, 3 . . . . . . . . . 104

9.4.4 Multiple bank switch - cycling between Bank sets 2, 3, 4 . . . . . . 105

9.5 Phase Noise Performance . . . . . . . . . . . . . . . . . . . . . 106

9.5.1 Phase Noise at upper and lower frequencies . . . . . . . . . . . . . 107

9.5.2 Phase noise over the frequency spectrum of oscillator . . . . . . . . . 108

9.5.3 Phase noise performance vs current consumption . . . . . . . . . . . . 109

9.6 Figure of Merit . . . . . . . . . . . . . . . . . . . . . 110

9.7 Measured Tuning Range of Oscillator . . . . . . . . . . . . . . . . . 110

9.8 Conclusion . . . . . . . . . . . . . . . . . . . . . . . 111

CHAPTER 10: ANALYSIS \& DISCUSSION . . . . . . . . . . . 112

10.1 Analysis of Transient Response . . . . . . . . . . . . . . . . . . . . 112

10.2 Analysis of Frequency Response . . . . . . . . . . . . . . . . . . . . . . 114

10.2.1 Frequency Response of Bank 5 to Bank 11 . . . . . . . . . . . . . . 119 
10.3 Linearity Analysis . . . . . . . . . . . . . . . . . . . . 119

10.3.1 Linearity analysis and transformation of multiple bank sets . . . . 120

10.4 Phase Noise Comparison _ . . . . . . . . . . . . . . . . . . . . . 121

10.5 FoM Comparison . . . . . . . . . . . . . . . . . . . 123

10.6 Conclusion . . . . . . . . . . . . . . . . . . . . 123

CHAPTER 11: EXTRA WORK . . . . . . . . . . . . . . 124

11.1 Frequency Divider to Route DCO Signal to DLF . . . . . . . . . . . . . . 124

11.2 Second Digital Controlled Oscillator to Verify Simulator Integrity . . . . . 127

11.3 DCO in 65nm Kit to Verify Theoretical Results . . . . . . . . . . . . . . 128

11.3.1 Tuning range of $65 \mathrm{~nm}$ oscillator . . . . . . . . . . . . . . . 130

11.4 Low Frequency Oscillator to Verify Theoretical Results . . . . . . . . . . . 130

11.4.1 Tuning range of the low frequency DCO . . . . . . . . . . . 131

11.5 Conclusion . . . . . . . . . . . . . . . . . . . 132

CHAPTER 12: FUTURE WORK . . . . . . . . . . . . . . . 133

12.1 Conclusion . . . . . . . . . . . . . . . . . 135

CHAPTER 13: THESIS SUMMARY . . . . . . . . . . . . . . 136

13.1 Conclusion . . . . . . . . . . . . . . . . . 137

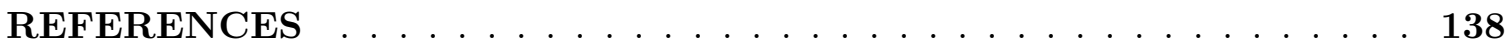

APPENDIX A: SCHEMATIC DIAGRAMS . . . . . . . . . . . . 142

APPENDIX B: BONDING AND PACKAGING . . . . . . . . . 146

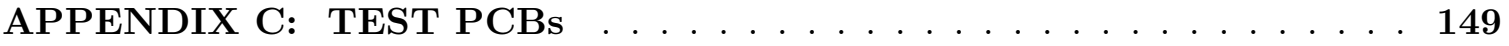

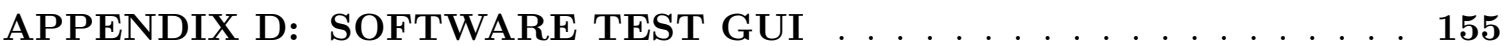




\section{LIST OF TABLES}

1 Tuning Range vs Linearity for Four Different Test Cases. . . . . . . . . . . . . 36

2 Number of Bank Sets, Banks, and Average Frequency Step Size. . . . . . . . . 43

3 Simulated Average Frequency Step Size for Each Bank Set. . . . . . . . . . . 70

4 Measured Frequency Step Sizes for the Bank Sets of the DCO. . . . . . . . . 100

5 Frequency Response Comparison . . . . . . . . . . . . . . . . . . 119

6 Linearity Comparison Between Theoretical, Simulated, and Measured Results. 119

7 Comparison Between New Theoretical DCO. . . . . . . . . . . . . . . 120

8 PN Comparison. . . . . . . . . . . . . . . . . . . . . 122

9 Comparison of Theoretical vs Simulated Results of 65nm Oscillator . . . . . 129

10 Comparison of Theoretical vs Simulated Results of Low Frequency Oscillator. 131 


\section{LIST OF FIGURES}

1 Model of PLL . . . . . . . . . . . . . . . . . . . 2

2 Model of ADPLL . . . . . . . . . . . . . . . . 3

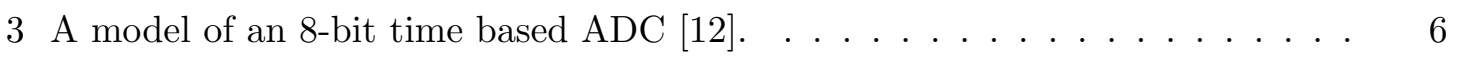

4 LMS calibration for VCO based ADC $[12] \ldots \ldots \ldots \ldots$

5 Normalized VCO transfer curve using LMS method [12] . . . . . . . . . . 8

6 VCO tuning curve using LMS method $[12] \ldots \ldots \ldots$. . . . . . . 8

7 Modified ICO . . . . . . . . . . . . . . . . . . . . . . . 9

8 Proposed VCO by Le et al. [14]. . . . . . . . . . . . . . . . . . . 10

9 VCO tuning curve of the VCO proposed by Le et al. [14] . . . . . . . . . . 10

10 Negative feedback in a) amplifier and b) VCO [5]. . . . . . . . . . . . . 11

11 Feedback loop model of DCO $[5] \ldots \ldots \ldots \ldots$

12 Feedback linearization in closed loop [5] . . . . . . . . . . . . . . . . 12

13 Switch capacitor $\mathrm{R}$ equivalence $[5] \ldots \ldots \ldots \ldots \ldots \ldots$

14 Results of DCO in Gorji et al. [5]. . . . . . . . . . . . . . . . . . . 13

15 Weighted varactors to offset effects on non-linearity [16] . . . . . . . 13

16 Second-order control system loop defining the function of an oscillator [35]. . 15

17 Parallel LC Resonator . . . . . . . . . . . . . . . . . . . . . . 16

18 LC oscillator dampens and decays after an impulse signal is applied to it [35]. 16

19 Negative resistance. . . . . . . . . . . . . . . . . . . 17

20 Popular way of implementing negative resistance. . . . . . . . . . . . 17

21 Implementation of negative gm oscillator using two transistors [35]. . . . . . 18

22 Small signal model of the circuit in Figure $21[35] \ldots \ldots$. . . . . . . . 18

23 Negative gm circuit. . . . . . . . . . . . . . . . . 20

24 Typical LC curve shape of L $=25 \mathrm{pH}, 100 \mathrm{pH}$, and $400 \mathrm{pH}$. . . . . . . . . 24

$25 \mathrm{R}^{2}$ values. . . . . . . . . . . . . . . . . . 28

26 Topographical map of $\mathrm{R}^{2} \ldots \ldots \ldots \ldots \ldots \ldots \ldots$

27 Linearity topographical 3D field map of frequency span of $1 \mathrm{GHz}$. . . . . . . 29

28 Field map of $\mathrm{R}^{2}$ for a frequency span of $1 \mathrm{GHz}(\mathrm{L}=470 \mathrm{pH}) . \quad \ldots \ldots . . .30$

29 Frequency vs bits of the DCO designed from Fig. 28. . . . . . . . . . . 32

30 A PMOS negative gm pair topology. . . . . . . . . . . . . . . . 32

31 Capacitance vs voltage curve of a typical varactor. . . . . . . . . . . . 33

32 All capacitors have fixed capacitance for equal frequency steps. . . . . . . . . 33

33 Relationship between linearity and tuning range. . . . . . . . . . . . . 37 
34 Relationship between linearity and tuning range from 3D perspective. . . . . 37

35 Schematic implementation of the LC DCO core. . . . . . . . . . . . . 39

36 Schematic implementation of DCO core buffers. . . . . . . . . . . . . . . 41

37 Example schematic of how an oscillator bank was implemented. . . . . . . . . 42

38 Alternative bank schematic. . . . . . . . . . . . . . . . . . . 42

39 Modelling bank capacitors and switch transistor using series RC. . . . . . . . 43

40 CML Schematic . . . . . . . . . . . . . . . . . . 45

41 Two stage method to reduce DCO digital control bits from 228 bits to 1 bit. 46

42 A 2-bit to 3 bit binary to thermometer decoder. . . . . . . . . . . 46

43 Schematic implementation of serial to parallel interface. . . . . . . . . . . 47

44 Schematic of a flip-flop used as a shift register. . . . . . . . . . . . . . 47

45 A 2 input mux using pass transistors. . . . . . . . . . . . . . . . . 48

46 Internal clock. . . . . . . . . . . . . . . . . . . . . . . . 49

47 DCO Core layout . . . . . . . . . . . . . . . . . . . 50

48 Design of an inductor intended to be used for the DCO . . . . . . . . . 51

49 An example layout of a digital bank. . . . . . . . . . . . . . . . 52

50 An example layout of a digital bank using multiple capacitors in parallel. . . 52

51 A layout of a CML used to divide the DCO frequency by half. . . . . . . . . 53

52 Layout of four CML dividers in series. . . . . . . . . . . . . . . . 54

53 Layout of large common drain buffer. . . . . . . . . . . . . . . 55

54 Layout of binary to thermometer decoder. . . . . . . . . . . . 56

55 Layout of 57 bit serial to parallel converter. . . . . . . . . . . . . 57

56 Layout of a single flip flop used in the serial to parallel converter. . . . . . . 58

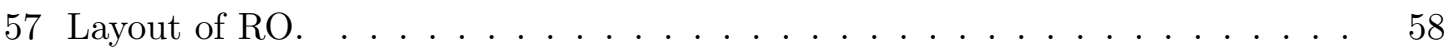

58 Layout of the DCO with digital compartments labelled. . . . . . . . . . 59

59 High resolution layout image of the DCO in Figure 58. . . . . . . . . . . 60

60 Layout of the second DCO. The size of the layout was $958 \mu m$ x $871 \mu m$. . 61

61 ADPLL full chip layout . . . . . . . . . . . . . . . . . . 62

62 Final layout. . . . . . . . . . . . . . . . . . . . . 63

63 Post layout transient simulations of DCO with all banks off. . . . . . . . . 65

64 Post layout transient simulations of DCO with all banks on. . . . . . . . . 66

65 Frequency simulations of stepping through Bank set 1. . . . . . . . . . 66

66 Frequency simulations of stepping through Bank set 2. . . . . . . . . . . 67

67 Frequency simulations of stepping through Bank set 3. . . . . . . . . . 67 
68 Frequency simulations of stepping through Bank set $4 \ldots \ldots$. . . . . . 68

69 Frequency simulations of stepping through Bank set $5 \ldots \ldots$. . . . . . 68

70 Frequency simulations of stepping through Bank set $6 \ldots \ldots$. . . . . . 69

71 Frequency simulations of stepping through bank set $7 \ldots \ldots$. . . . . . . 69

72 The simulated phase noise of the oscillator when all banks are turned off. . . 71

73 The simulated phase noise of the oscillator when all banks are turned on. . . 71

74 Simulated PN over frequency span of DCO . . . . . . . . . . . . . . 72

75 Simulated PN for various current draw. . . . . . . . . . . . . . . . . . 72

76 The phase noise performance at the output of four CML dividers in series. $\quad$. 73

77 Figure of merit over DCO tuning range. . . . . . . . . . . . . . . . 74

78 FoM vs current. . . . . . . . . . . . . . . . . . . . . 74

79 Transient simulation of CML divisions. . . . . . . . . . . . . . . 75

80 Transient simulation of the ring oscillator frequency divided by $50 \ldots \ldots$

81 Images of the ADPLL chips containing the DCO that was fabricated. . . . . 77

82 Bottom view of die. . . . . . . . . . . . . . . . . . . 78

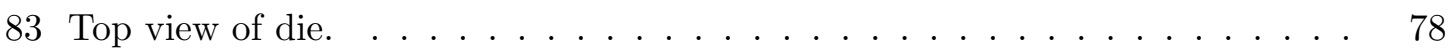

84 Right side view of die. . . . . . . . . . . . . . . . . . . . 79

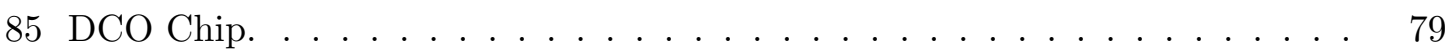

86 Die bonding diagram. . . . . . . . . . . . . . . . . . 80

87 The PCB design of the main test board for the DCO. . . . . . . . . . . . 81

88 Main test PCB with all the components soldered on (top view). . . . . . . . 82

89 The main test PCB with all components soldered onto it (side view). . . . . 83

90 The digital testing protocol/interface of the DCO . . . . . . . . . . 83

91 The digital testing protocol of the DCO . . . . . . . . . . . . . 84

92 A 4-bit DCO test PCB digital interface. . . . . . . . . . . . . . . 84

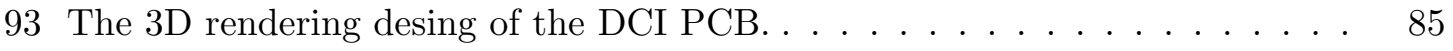

94 The DCI-DCO controller PCB with all components soldered. . . . . . . . . 85

95 Slightly modified (version 2) DCI PCB with all components soldered. . . . . 86

96 Graphical User Interface (GUI) of the software created to auto test the DCO. 87

97 Test PCBs for debugging the DCO. PCBs designed by the author of this thesis. 88

98 Equipment test setup for evaluating the fabricated DCO. . . . . . . . . . 89

99 PCB test setup for measuring the performance of the DCO . . . . . . . . . . 90

100 Measured transient signal of DCO when all banks off. . . . . . . . . . . . . . 91

101 Measured transient signal of DCO when all banks on. . . . . . . . . . . . . 92 
102 Signals of the CML being divided by $2 \ldots \ldots \ldots$. . . . . . . . . . 92

103 Divided ring oscillator output at maximum frequency. . . . . . . . . . . 93

104 Divided ring oscillator output at minimum frequency. . . . . . . . . . . . . 93

105 FFT signal (divide by 8) when all banks of the DCO is off. . . . . . . . . . 94

106 FFT signal (divide by 8) when all banks of the DCO is on. . . . . . . . . . 94

107 FFT plot of all the bank steps of Bank set 1 overlaid. . . . . . . . . . . . . 95

108 FFT plot of all the bank steps of Bank set 2 overlaid. . . . . . . . . . . . . 95

109 The measured frequency response of Bank 1 steps. . . . . . . . . . . . 96

110 The measured frequency response of Bank set 2 at the upper frequency limit. 97

111 The measured frequency response of Bank set 2 at a lower frequency limit. . $\quad 97$

112 The measured frequency response of Bank set 3 at the upper frequency limit. 97

113 The measured frequency response of Bank set 3 at a lower frequency limit. . 98

114 The measured frequency response of Bank set 4 at the upper frequency limit. 98

115 The measured frequency response of Bank set 4 at a lower frequency limit. . 99

116 The measured frequency response of Bank set 5 at the upper frequency limit. 99

117 The measured frequency response of Bank set 5 at a lower frequency limit. . 99

118 An example of frequency settling time required by banks in Bank Set 1. . . 101

119 An example of frequency settling time required by banks in Bank Set 2. . . 101

120 An example of frequency settling time required by banks in Bank Set 3. . . 102

121 An example of frequency settling time required by banks in Bank Set 4. . . 102

122 The frequency response of stepping through of Bank sets 1 and 2 . . . . . 103

123 Banks 1 and 2 with duplicate combinations removed. . . . . . . . . . . 103

124 The frequency response of stepping through Banks sets 1, 2, and 3. . . . . 104

125 Banks 1, 2, and 3 with duplicate combinations removed. . . . . . . . . . . 104

126 The frequency response of stepping through Bank sets 2 and 3. . . . . . . 105

127 Banks 2 and 3 with duplicate combinations removed. . . . . . . . . . . . 105

128 The frequency response of stepping through Bank sets 2, 3, and 4. . . . 106

129 Banks 2, 3, and 4 with duplicate combinations removed. . . . . . . . . 106

130 Measured PN when all banks off. . . . . . . . . . . . . . . . . . 107

131 Measured PN when all banks on. . . . . . . . . . . . . . . . . 107

132 Measured PN over frequency range of DCO . . . . . . . . . . . . . . 108

$133 \mathrm{PN}$ at oscillator frequency. . . . . . . . . . . . . . . . . . 108

134 Measured PN vs current consumption; all banks off. . . . . . . . . . . . . . 109

135 Measured PN vs current consumption; all banks on. . . . . . . . . . . . . . 109 
136 FoM of DCO vs various current consumption when all banks are off. . . . . 110

137 FoM of DCO vs various current consumption when all banks are on. . . . . 110

138 Simplified parasitic model between CML output and oscilloscope. . . . . . . 112

139 Test simulation of the parasitic model. . . . . . . . . . . . . . . . . 113

140 Test bench for simulating the parasitic model. . . . . . . . . . . . . . . . 113

141 Simulation of Bank 1 with (orange) and without RC extraction (purple). . . 114

142 Metal fills around core. . . . . . . . . . . . . . . . . . . 116

143 Parasitic due to metal fill. . . . . . . . . . . . . . . . . 116

144 LC core with parasitic included. . . . . . . . . . . . . . . . 117

145 LC Curve showing frequency span. . . . . . . . . . . . . . . . . 118

146 A theoretical DCO from $6.66 \mathrm{GHz}$ to $5.59 \mathrm{GHz} \ldots \ldots \ldots$. . . . . . . 120

147 Simulated Q of DCO Core. . . . . . . . . . . . . . . . . 121

148 Signal propagating from CML to $(\div 2-39)$ dividers. . . . . . . . . . . . . . 124

149 Layout of the divider block. . . . . . . . . . . . . . . . . 125

150 Divider control signals and expected output frequencies. . . . . . . . . . 125

151 Division by $19 . \ldots \ldots \ldots \ldots \ldots \ldots \ldots \ldots$

152 Division by $18 \ldots \ldots \ldots \ldots \ldots \ldots \ldots \ldots \ldots$

153 Division by $17 \ldots \ldots \ldots \ldots \ldots \ldots \ldots \ldots \ldots$

154 Division by $16 \ldots \ldots \ldots \ldots \ldots \ldots \ldots \ldots \ldots \ldots$

155 Frequency response of DCO 2 when all banks are off. . . . . . . . . . . 128

156 Layout of DCO in $65 \mathrm{~nm}$ (TSMC kit). . . . . . . . . . . . . . 129

157 Frequency simulation results of $65 \mathrm{~nm}$ DCO . . . . . . . . . . . . 129

158 Schematic design of low frequency DCO . . . . . . . . . . . . . 130

159 Frequency simulation of a low frequency oscillator. . . . . . . . . . . . . 131

160 Transient response of the low frequency DCO . . . . . . . . . . . . 131

161 Linearization calibration scheme. . . . . . . . . . . . . . . . . . 134

162 Sample model of the linearization algorithm. . . . . . . . . . . . . . . 134

163 Example simulation of the linearization algorithm. . . . . . . . . . . 135 


\section{LIST OF ACRONYMS}

ADC analog-to-digital converter

ADPLL all-digital phase-locked loop

BJT bipolar junction transistor

BW bandwidth

CCO current-controlled oscillator

CML current mode logic

CMOS complementary metal oxide semiconductor

DC direct current

DCI digital-controller-interface

DCO digitally controlled oscillator

DLF digital loop filter

DOE Department of Electronics

DSP digital signal processing

EMX electromagnetic simulator software

FFT fast fourier transform

FM frequency modulation

FoM figure of merit

GND ground

GUI graphical user interface

HB harmonic balance

IC integrated circuit

ICO current-controlled oscillator

LC inductor-capacitor

LMS least mean square

MASc Masters of Applied Science

MOSFET metal-oxide-semiconductor field-effect-transistor

MS Microsoft

NMOS n-type metal-oxide-semiconductor 


\begin{tabular}{ll} 
PC & personal computer \\
PCB & printed circuit board \\
PMOS & p-type metal-oxide-semiconductor \\
PN & phase noise \\
PSS & periodic steady state \\
QFN & quad-flat no leads \\
QFP & quad-flat package \\
RC & resistor-capacitor \\
RLC & resistor-inductor-capacitor \\
S2PI & serial to parallel interface \\
SoC & system on a chip \\
SPI & serial to peripheral interface \\
SR & set-reset \\
TDC & time to digital converter \\
TR & tunning range \\
TSMC & Taiwan Semiconductor Manufacturing Company \\
TT & typical-typical \\
VCO & voltage-controlled oscillator \\
VDD & supply voltage \\
\hline
\end{tabular}


This page has been left blank intentionally. 


\section{CHAPTER 1: INTRODUCTION}

The reason for this report is to satisfy the requirements for the MASc graduate degree at the Department of Electronics (DOE) from Carleton University, and the purpose of this report is to methodologically observe and design an extremely linear digitally controlled oscillator (DCO) for use in all-digital phase-locked loop (ADPLL). This report includes an introduction that discusses the overview of this report and the motivation for the research topic, a section on research theory and technique, a section on simulated results, a section on the observation, findings, and results of this research, a section on the analysis of the results, a conclusion, and references.

\subsection{Oscillators and Phase-Locked Loops}

An oscillator is a device, method, means, or an instigator that periodically produces something, and sustains that periodicity over the duration of its usefulness. An electronic oscillator is a device or circuit that generates electrical signals that are periodic such as square waves, sine waves, saw-tooth waves, or triangle waves [1]. Electronic oscillators in one form another are used in almost every electronic devices today, whether it be in power sources such as buck-boost converters, personal computers (PCs), radios and consumer goods, or whether it be used in modern day warfare equipment such as radars and sonars. In fact modern technology, and henceforth modern civilization (due to its contemporary social standards, affluence, and being), arguably could not exist without the electronic oscillator. One could even argue that it is this invention "the electronic oscillator" that gave rise to mankind's welfare and the unprecedented global technological "marvelation" and achievement that exists today. The backbone of all modern communications and computational prowess is the ticking clock or synthesizer. Clocks are quite aimless to electrical communication unless they can be synchronized for their intended purpose(s). The clocks can be synchronized through the use of some means such as locked loops. Usually, at the heart of these locked loops such as a phase-locked loop (PLL) is the oscillator. It is this oscillator for use in PLL that will be discussed in the following thesis.

\subsubsection{Analog Phase-Locked Loops}

An example of a generic analog phase-locked loop is shown in Figure 1. The main parts of the PLL consists of: a phase detector, a loop filter, and an oscillator. The phase detector detects the phase difference between the reference frequency and any drift from the oscillator 
frequency, and sends the difference to the loop filter. The loop filter removes the high frequency components and leaves a clean direct current (DC) signal that can be further used to control the oscillator frequency drift in a regulated manner [2]. The phase-locked loop is a negative feedback system that keeps the oscillator frequency in check from digressing from its intended operational output. For most of history the analog PLLs have been the more dominant architecture used in electronics industry. Recently with the advent of system on a chip (SoC) technology, and the shrinking of nanometer $(\mathrm{nm})$ process, all-digital phase-locked loops have become more recognizable and ever more popular. The ADPLL is the digital counterpart to the more traditional analog phase-locked loop and is gaining traction in the engineering community.

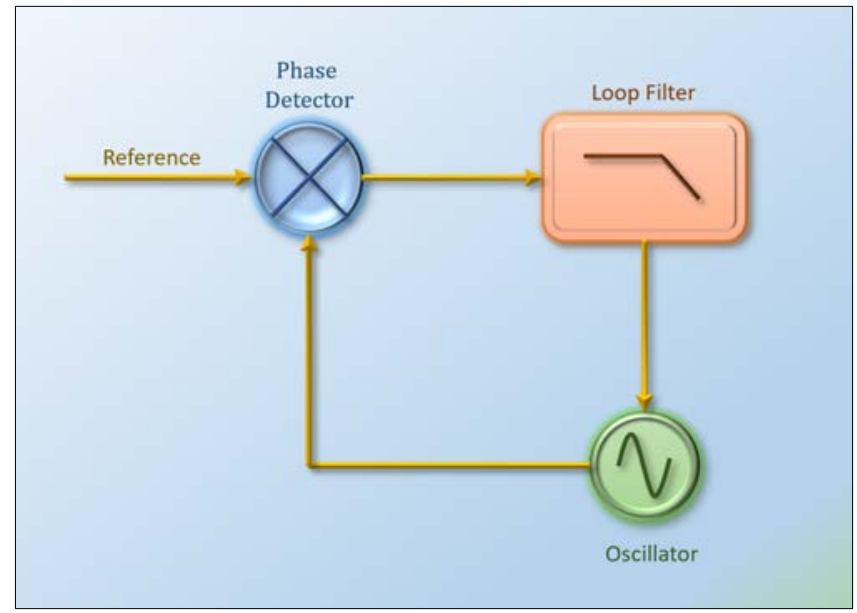

Figure 1: Generic model of an analog PLL consisting of a phase detector, a loop filter, and an oscillator.

\subsubsection{All-Digital Phased-Locked Loop}

An example of the all-digital phase-locked loop is shown in Figure 2. An all-digital PLL loop may consist of an integer counter, a time to digital converter (TDC), a digital loop filter (DLF), and a digitally controlled oscillator. The all digital loop may also contain dividers in the oscillator pathway to lower the operational computational cycle for the TDC and counter (the analog PLL may also contain dividers in the frequency pathway in Figure 1 ; although it was omitted for the sake of simplicity). The integer counter and the time to digital converter converts the time domain information (and accordingly frequency) into digital code, which is then past onto the digital loop filter. The digital loop filter operates on the digital information and then sends a digital signal to the digitally controlled oscillator. 


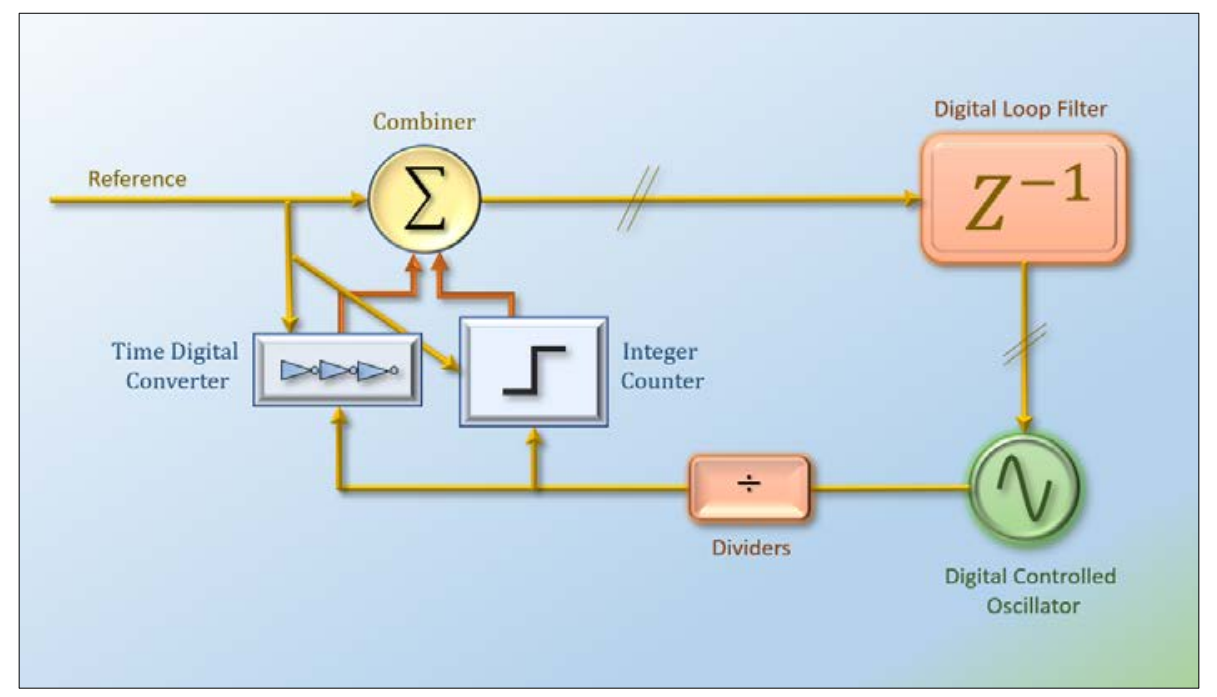

Figure 2: Model of an ADPLL consisting of an integer counter, a time to digital converter, a digital loop filter, and a digitally controlled oscillator.

The oscillator frequency is again then re-sampled by the TDC closing the loop of the system. The ADPLL reiterates these steps over and over again keeping the oscillator from drifting from its intended operational frequency.

\subsection{Motivation for Thesis Research}

There are many aspects of the oscillator that are crucial for the proper functioning of the locked loop. For example the bandwidth (BW) of a second order PLL will be heavily dependent on the loop gain and will vary drastically if the oscillator is non-linear, making system parameters very difficult to predict [3]. For smooth locking to occur in an all-digital phase-locked loop, the output frequency must be linear and monotonic [4]. Linearity is often overlooked when designing oscillators (whether for voltage-controlled oscillators (VCOs) or digitally controlled oscillators) due to the complexity of overcoming such a challenge [5]. Unfortunately nonlinear oscillators (depending on the application it is used for) can have large impacts in predicting system behavioural parameters. Oscillators in their inherent nature are nonlinear with respect to frequency. LC (inductor-capacitor) oscillators are the product of the solution to an LC network [6], ring oscillators are product of the delay between each stage and the total number of units [5], and RC (resisotr-capacitor) oscillators are a product of the $\mathrm{RC}$ charge/discharge time constant [10], which are all fundamentally governed by non-linear physics. Linearizing oscillators is still a niche topic during this modern era, for this reason the topic will be analysed furthermore in this thesis. 


\subsection{Thesis Scope and Research Contribution}

This thesis report focuses on designing a linearized digitally controlled oscillator (where the output frequency is linearized with respect to the input control bits) for its use in an all-digital phase-locked loop. More specifically, the research looks at designing LC digitally controlled oscillators with high linearity using a different outlook and new perspective that has not been previously explored (to best knowledge of the author). The paper analyzes LC behaviour using statistics and a new concept known as linearity field maps. Furthermore the technique proposed in this paper for an LC oscillator is robust and applicable for all LC topologies, and over any technology. It can potentially be used for RC and ring oscillators as well if the same methodology described in this paper (derived from first principles) is used for their respective topologies. As with all architectures, there are limitations to the technique proposed (keeping exceptional linearity over a vast and wide tuning range) however when applicable, the method is very powerful, it is conceptually easy to understand and implement, and produces extreme linearity. The thesis contributions are:

- Introducing a new theoretical concept known as 'topographical' linearity field maps using regression $\left(R^{2}\right)$ as a tool.

- Using $R^{2}$ as a Figure of Merit for linearity.

- Theoretical design of linear DCO using the linearity field maps.

- Implementing theoretical linear DCO using a cascading pyramid scheme of equal banks within equal banks to achieve the linearity.

\subsection{Thesis Organization and Outline}

The thesis includes an introduction that discusses the motivation of the thesis in Chapter 1. Chapter 2 discusses any prior literature regarding and related to the current work in this thesis. Chapter 3 discourses on the theory of oscillation, more specifically on negative gm oscillators. Chapter 4 introduces to the theorem used for the work of this thesis. The theorem and its analysis is part of the contribution of this thesis. Chapter 5 deals with the schematic design of the oscillator. Chapter 6 illustrates the drawn layouts of the oscillator and its peripheral circuitry. Chapter 7 presents the simulated results of the oscillator. Chapter 8 dilates on the test set-up used to assess the performance of the oscillator built for this thesis. Chapter 9 presents the obtained measurements of the oscillator. Chapter 10 analyses and discusses the obtained results from Chapter 9. Chapter 11 talks about 
any extra work that has been done during the course of this thesis. Chapter 12 addresses any future work that is still left to do regarding the oscillator and topics related to it, and Chapter 13 summarizes and concludes the thesis.

\subsection{Conclusion}

The introductory chapter discussed the reason and purpose of this thesis. The chapter gave some background on oscillators and phase-locked loops, and then introduced what an all-digital phase-locked loop was. The chapter also discussed the motivation for the thesis, the thesis scope, research contribution, and briefly laid out the structure of the thesis. 


\section{CHAPTER 2: PRIOR LITERATURE}

As stated previously in Chapter 1.2, linearizing oscillators, and more specifically linearizing digitally controlled oscillators, is still a small métier in the electronics industry. It should be noted that when talking about linearity, for a VCO it is generally referring to linearizing the input voltage vs the output frequency of the oscillator while for a DCO it is referring to linearizing the input digital control bits vs the output frequency. Finding recent research on linearizing DCOs, or atleast recent publications that are accessible and undisclosed is quite challenging; nevertheless there have been plenty of papers in the fast few decades that have touched on the subject on how to linearize oscillators.

There have been solutions that proposed to linearize oscillators using closed loop methods (akin to feedback loops of PLLs) [5], solutions that proposed to linearize oscillators using open loop methods that directly interfere with and modify the core characteristic of the oscillator [19], and solutions that use advanced digital signal processing (DSP) and analog-to-digital converters (ADCs) to linearize the analog core characteristic of the oscillator [11].

There have also been a few statistical approaches proposed to optimize linearity such as [7] and [4] using least mean square (LMS) and standard deviation $(\sigma)$ as the figure of merit. The next few sections will look at a few papers on how they tried to linearize the frequency response in an oscillator.

\subsection{Least Mean Square Calibration Method}

H. Venkatram et al. (2009) [12] proposes to linearize VCO non-linearity using an LMS (least mean square) algorithm. The paper presents an 8-bit $10 \mathrm{MHz}$ Nyquist Rate VCO based ADC (analog to digital converter) that uses a calibration technique. An example of an 8-bit time based ADC is shown in Figure 3.

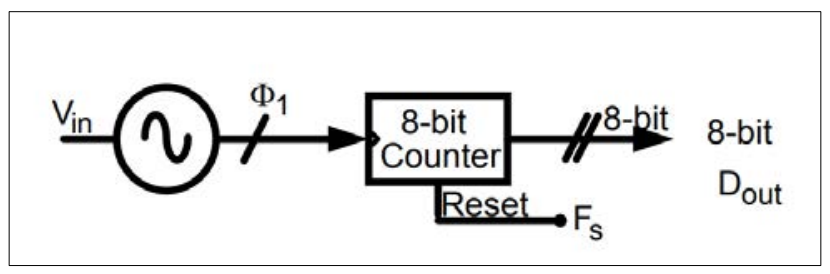

Figure 3: A model of an 8-bit time based ADC [12]. 
The ADC uses an 8-bit coarse binary counter which is clocked by the VCO and is reset by the sampling clock. It takes the tuning voltage to phase equation:

$$
\Phi_{V C O}(n)=2 \pi \int_{n T_{s}}^{\left(n T_{s}+T_{s}\right)}\left(K_{V C O} v_{i n} \sin \left(\omega_{i n} t\right)+f_{r e f}\right) d t
$$

and tries to model it by the equation:

$$
\Phi_{V C O}(n)=\alpha v_{i n}+\beta v_{i n}^{2}+\gamma v_{i n}^{3}+\phi_{e}(n)
$$

where $\alpha, \beta$ and $\gamma$ are constraints that models the non-linearity of the $\mathrm{VCO}$ and $\phi_{e}(n)$ models the quantization error from the VCO based ADC. For a given set of points $\left(x_{1}, y_{1}\right),\left(x_{2}, y_{2}\right)$ $\ldots\left(x_{n}, y_{n}\right)$, the best fit line using LMS can be derived from:

$$
\left[\begin{array}{c}
y_{1} \\
y_{2} \\
\vdots \\
y_{n}
\end{array}\right]=\left[\begin{array}{c}
x_{1} \\
x_{2} \\
\vdots \\
x_{n}
\end{array}\right] \cdot[m]
$$

where $m$ is evaluated by:

$$
m_{L M S}=\sum_{i=i}^{N} x_{i} y_{i} / \sum_{i=1}^{N} x_{i}^{2}
$$

The LMS equation is applied using ADCs [13] where the radixes of ADCs are the parameters of interest. A slow and accurate $\mathrm{ADC}$ quantizes $v_{\text {in }}$ which is then compared with the output from another ADC under calibration shown in Figure 4.

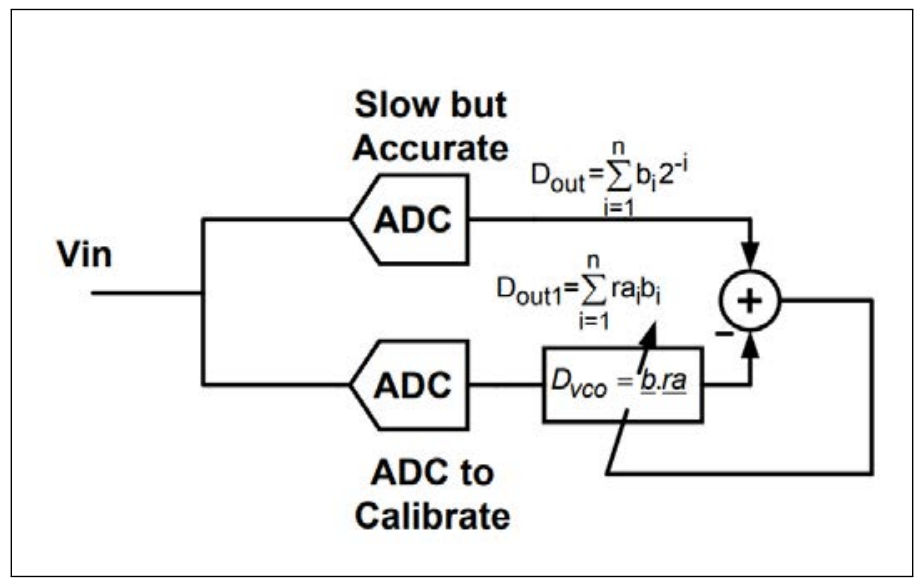

Figure 4: LMS calibration for VCO based ADC [12].

The values $v_{\text {in }}$ and $v_{\text {in }}{ }^{2}$ is approximated by $D_{\text {out }}$ and $D_{\text {out }}{ }^{2}$ from the slow ADC while 
$\Phi_{V C O}(n)+\phi_{e}$ is obtained from the VCO ADC output code. The VCO architecture used in the literature was an 8-stage differential ring. Since the VCO architecture already had a sinc filtering nature, the higher order terms were ignored. The resulting normalized calibrated VCO transfer curve (using LMS )is shown in Figure 5 and the simulated frequency response of the VCO is shown in Figure 6.

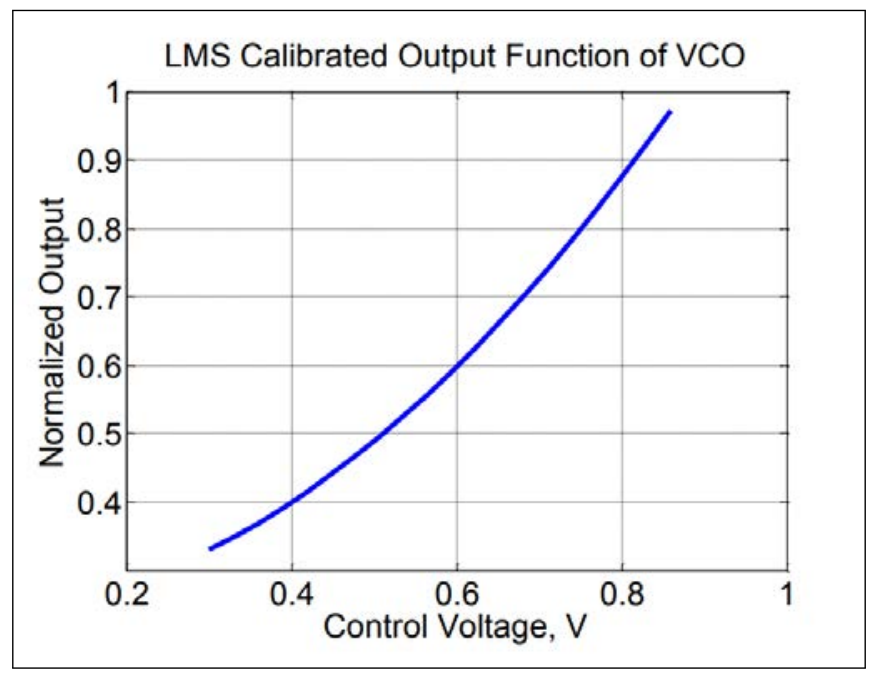

Figure 5: Normalized VCO transfer curve using LMS method [12].

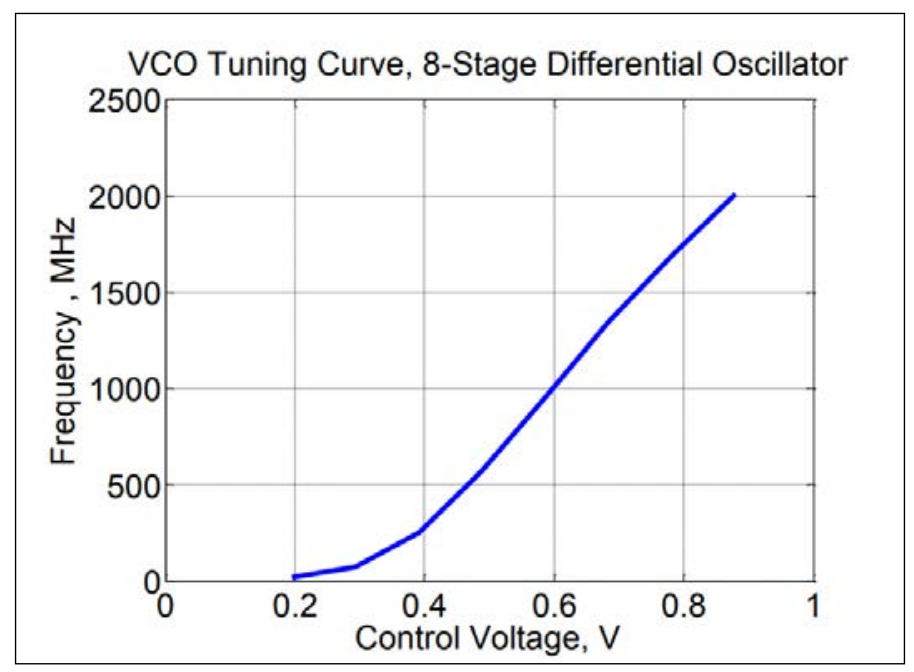

Figure 6: VCO tuning curve using LMS method [12].

\subsection{High Linearity VCO Using Modified ICO}

N. P. T. Le et al. (2009) [14] uses a modified current-controlled oscillator (ICO) to linearize the input voltage that controls the output frequency; it uses a voltage controlled current source to control the VCO. An example of a modified ICO is shown in Figure 7. 


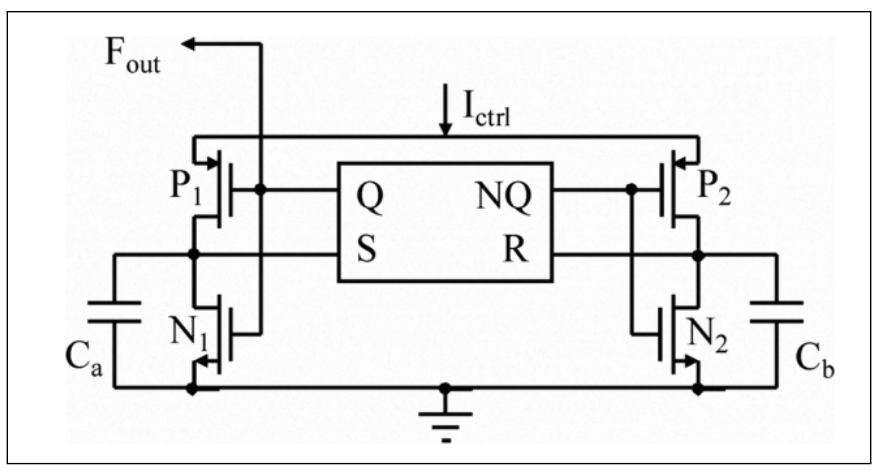

Figure 7: Modified ICO using SR (set-reset) latch consuming less power than traditional ICOs [14].

Le et al. points out that the period of the output signal is mainly due to the capacitor charge times $\left(t_{c}\right)$ and latch delay times $\left(t_{d}\right)$; where the frequency of the oscillator can be written as:

$$
f=\frac{1}{T}=\frac{1}{2\left(t_{c}+t_{d}\right)}=\frac{I_{c t r l}}{2\left(C \cdot V_{t h}+t_{d} \cdot I_{c t r l}\right)}
$$

The value 2 in the denominator of the equation above comes from charging both $C_{a}$ and $C_{b}$ alternatively, and $V_{t h}$ is the threshold voltage of the transistors in Figure 7 . It can be seen that the output frequency is non-linear due to the $t_{d} \cdot I_{c t r l}$ term. Le et al. proposes a modified feedback loop (by using switched-capacitor based frequency-to-voltage converters shown in Figure 8), where the transfer curve then becomes:

$$
V_{\text {in }}=V_{\text {feedback }}=R_{1} \cdot C_{1} \cdot V_{\text {ref }} \cdot f
$$

If $V_{\text {ref }}$ is chosen to be $-1 \mathrm{~V}$, then the above equation becomes:

$$
V_{\text {in }}=V_{\text {feedback }}=R_{1} \cdot C_{1} \cdot f
$$

The input control voltage $\left(V_{\text {in }}\right)$ now has been linearized with $f$. 


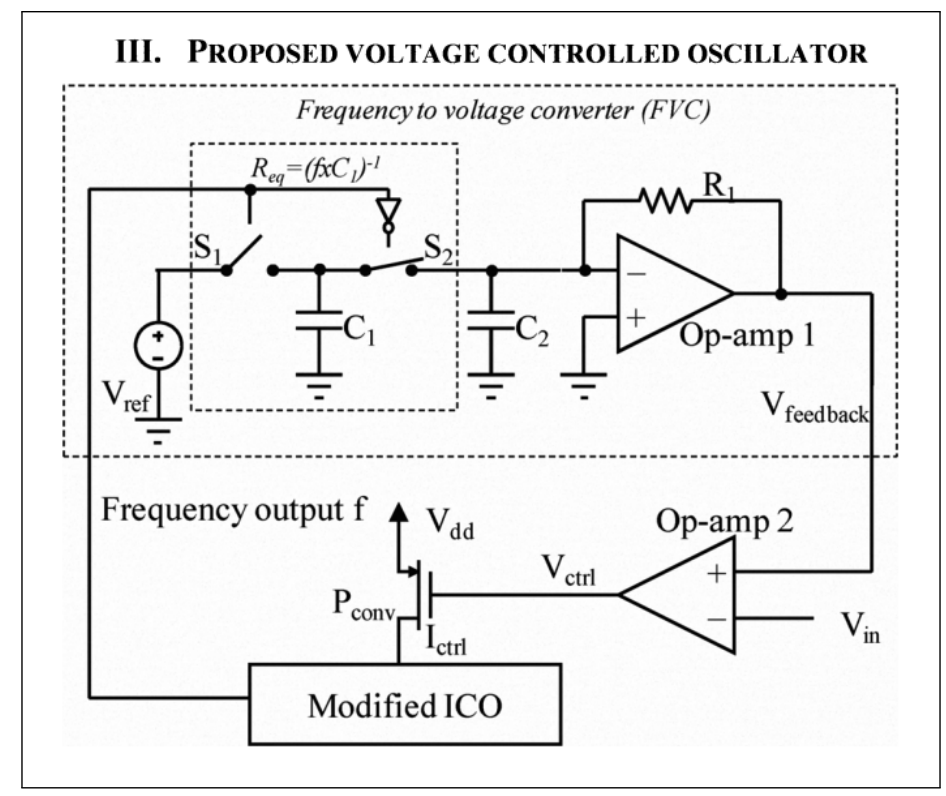

Figure 8: Proposed VCO by Le et al. [14].

The results of the proposed oscillator with the feedback loop by Le et al. is shown in Figure 9.

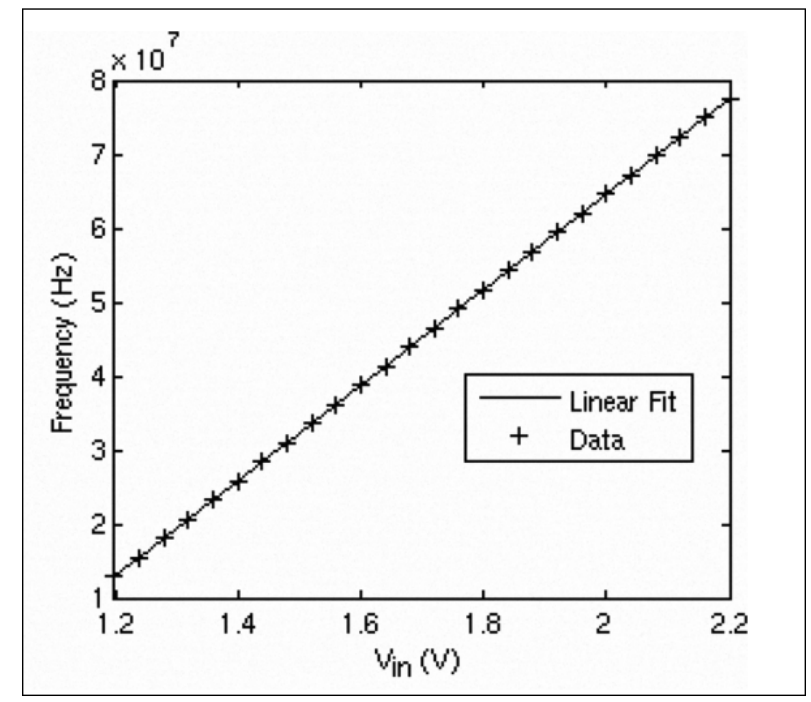

Figure 9: VCO tuning curve of the VCO proposed by Le et al. [14]

\subsection{Highly Linear DCO in 65-nm CMOS}

J. Gorji et al. (2017) [5] uses a current feedback system to linearize a differential ring oscillator. Figure 10 shows a negative feedback loop in a) an amplifier and b) a VCO. 


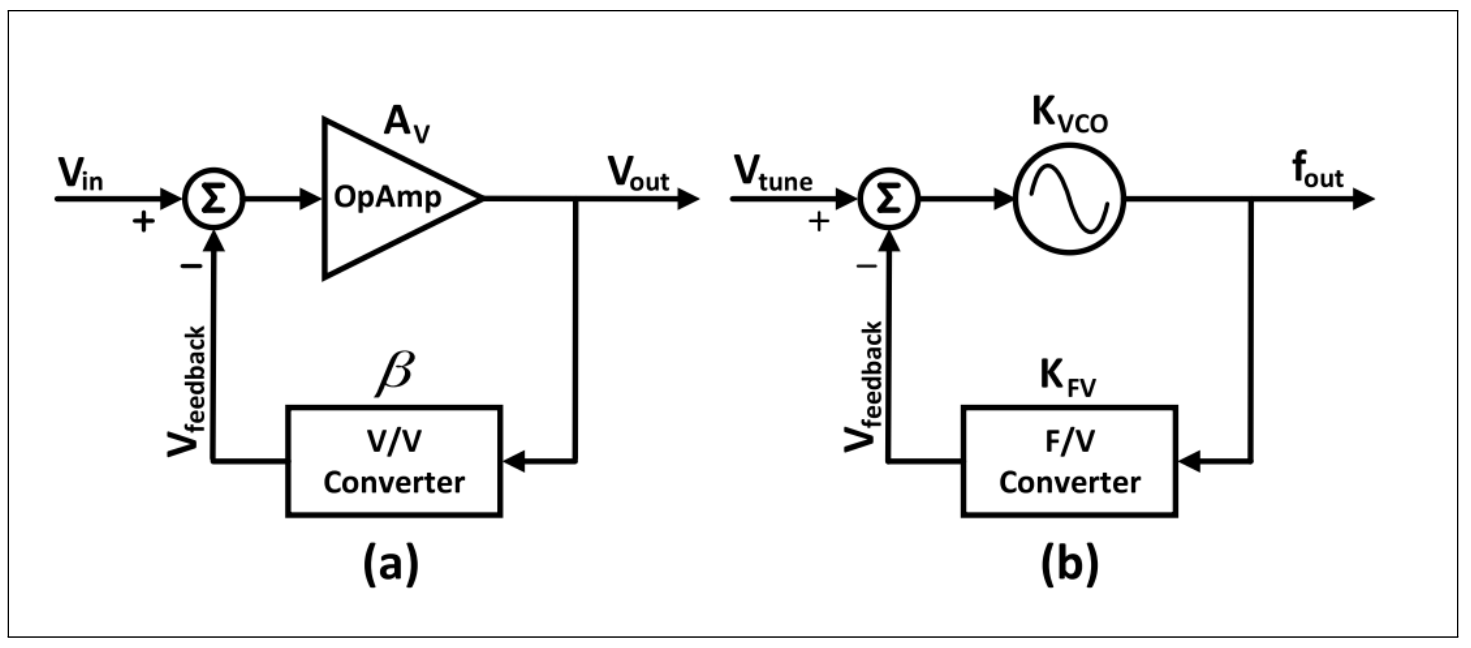

Figure 10: Negative feedback in a) amplifier and b) VCO [5].

Gorji uses the realization that if the loop gain $A \beta$ is large, then the total gain of the feedback system is independent of the amplifier transfer function (A) and is dependent on the inverse transfer function $(1 / \beta)$ :

$$
\left.A\right|_{\text {feedback }}=\frac{V_{\text {out }}}{V_{\text {in }}}=\frac{A}{1+A \beta} \approx \frac{1}{\beta}
$$

Gorji then extends the above idea in [5] (regarding the loop of the amplifier) to a current controlled oscillator feedback loop (shown in Figure 11) where:

$$
\left.K_{C C O}\right|_{\text {feedback }}=\frac{f_{\text {out }}}{I_{\text {tune }}}=\frac{K_{C C O}}{1+K_{C C O} \cdot K_{F I}} \approx \frac{1}{K_{F I}}
$$

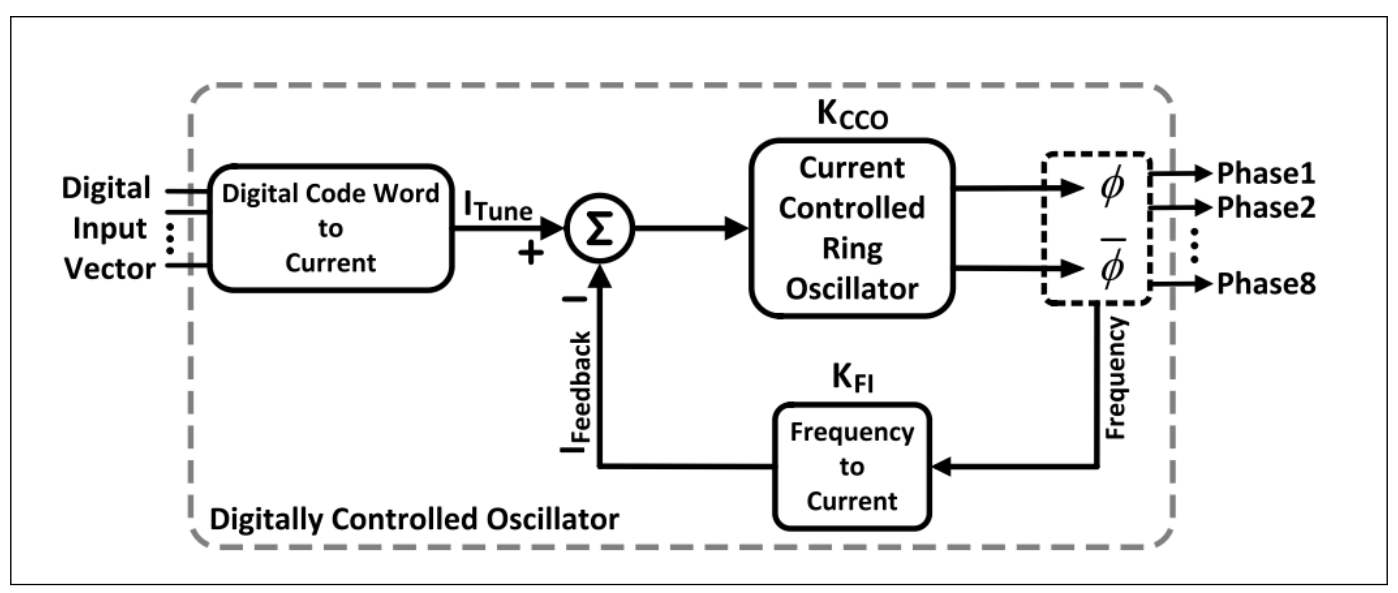

Figure 11: Feedback loop model of DCO [5]. 
Figure 12 shows a conceptual model of the feedback-assisted DCO.

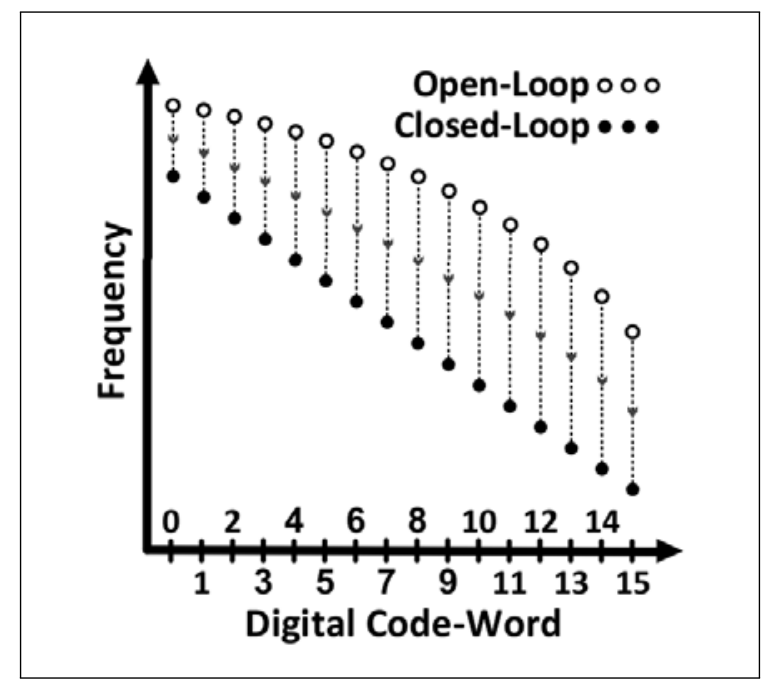

Figure 12: Feedback linearization in closed loop [5].

Gorji et al. claims that because the feedback loop is contingent on the oscillator output ( $I_{\text {tune }} / I_{\text {feedback }}$ ratio is more pronounced in higher frequencies than in lower frequencies), the frequency will be more reduced for lower frequencies in a closed-loop system. As a result the DCO characteristic curve will be linearized (see Figure 12).

Gorji et al. uses switch capacitance to convert frequency into current (see Figure 13) where:

$$
\begin{gathered}
I_{O U T} \approx \frac{V_{R E F}}{R_{e q u}}=V_{R E F} \cdot C_{s} \cdot f_{O S C} \\
K_{F I}=\frac{I_{O U T}}{f_{O S C}}=V_{R E F} \cdot C_{S}
\end{gathered}
$$

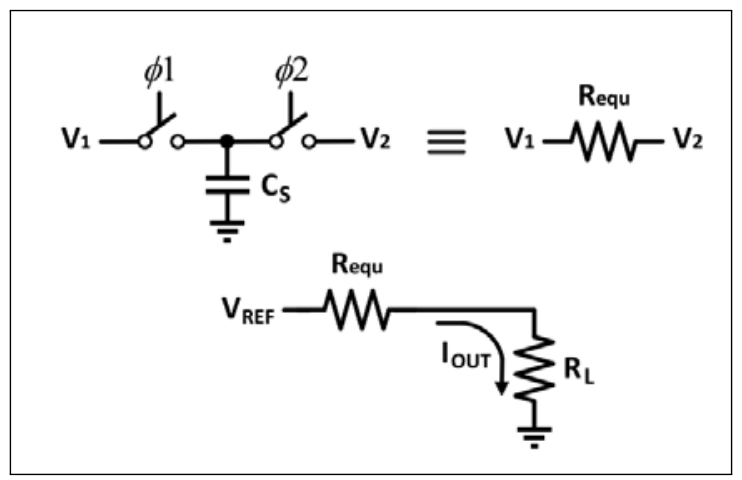

Figure 13: Switch capacitor R equivalence [5]. 
It can be seen that substituting $K_{F I}$ into the feedback expression results in:

$$
\left.K_{C C O}\right|_{\text {feedback }} \approx \frac{f_{O S C}}{I_{O U T}}
$$

The output frequency is now proportional to the oscillator gain. Therefore the oscillator frequency has been linearized. The results of Gorji et al. DCO is shown in Figure 14.

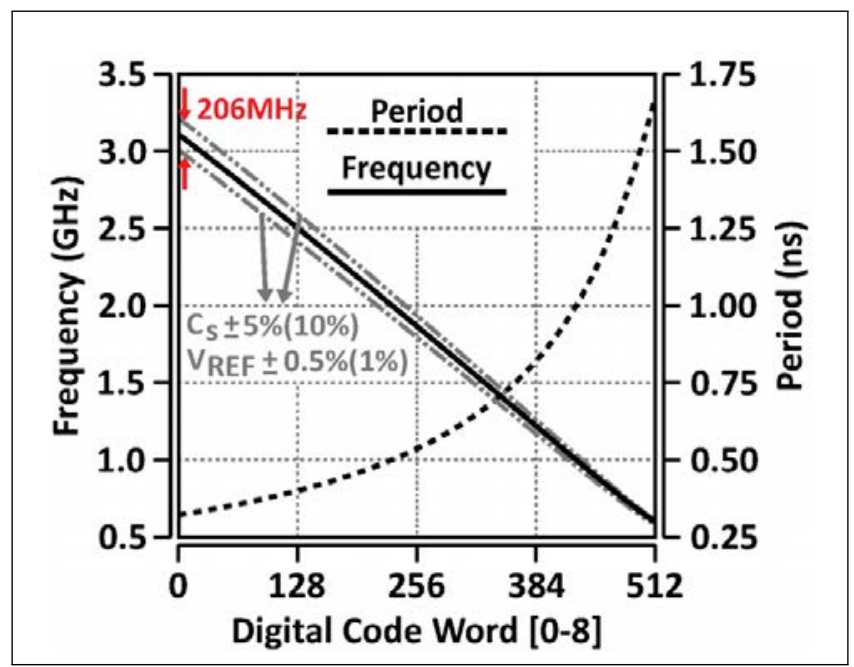

Figure 14: Results of DCO in Gorji et al. [5].

\subsection{Other Literature in Linearizing Frequency Response of Oscillators}

There have been other techniques proposed in literature to linearize the frequency response of oscillators; such as using weighted banks from [15] and [16] (shown in Figure 15), or controlling the potential of the IC (integrated circuit) substrate to correct linearity from [17].

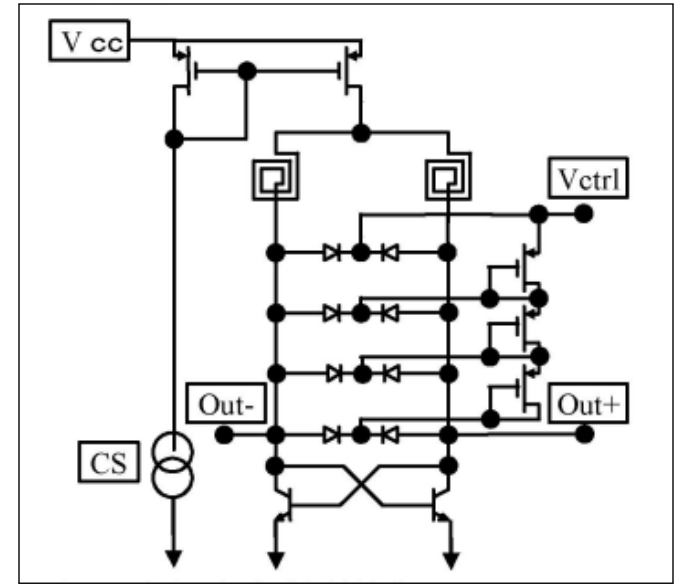

Figure 15: Weighted varactors to offset effects on non-linearity [16]. 
Reference [7] used iterative LMS to optimize tuning bandwidth and linearity. See references [18] - [32] for more literature on oscillator frequency linearity.

\subsection{Conclusion}

This chapter discussed prior literatures on how to linearize the frequency response of an oscillator; including papers from H. Venkatram et al. which used an LMS calibration method. N. P. T. Le et al. used a feedback loop method on a modified current-controlled oscillator, and J. Gorji et al. used a feedback loop method on a differential ring oscillator architecture to linearize the frequency response. 


\section{CHAPTER 3: OSCILLATION THEORY}

This chapter will look at the theory behind the oscillation of an oscillator.

\subsection{Oscillators and Barkhausen Criterion}

An oscillator can be described by the second order control system model shown in Figure 16, where:

$$
\frac{V_{\text {out }}(s)}{V_{\text {in }}(s)}=\frac{H_{1}(s)}{1-H_{1}(s) H_{2}(s)}
$$

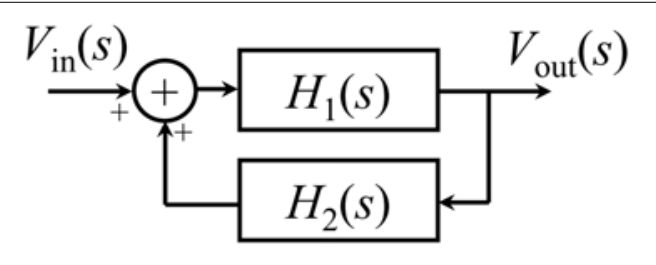

Figure 16: Second-order control system loop defining the function of an oscillator [35].

If the magnitude of $H_{1}(j \omega) H_{2}(j \omega)$ is unity (where $s=j \omega$ ), and the phase changes by a radian of $2 n \pi$, then the oscillator has met the conditions for oscillation and will oscillate. These properties are collectively known as the Barkhausen criterion where:

$$
\begin{aligned}
& \left|H_{1}(j \omega)\right|\left|H_{2}(j \omega)\right|=1 \\
& \angle H_{1}(j \omega) H_{2}(j \omega)=2 n \pi
\end{aligned}
$$

If the magnitude is less than unity $\left(H_{1}(j \omega) H_{2}(j \omega)<1\right)$, then oscillator will dampen and die out, and if the magnitude is greater than $\left(H_{1}(j \omega) H_{2}(j \omega)>1\right)$, then the oscillator magnitude will increase until it is either limited by the technology and power supply, or it breaks the system. This criterion is valid for any oscillator whether it be ring oscillators, $\mathrm{RC}$ oscillators, or LC oscillators.

\subsection{Parallel LC oscillators}

LC oscillators are oscillators that have a resonator of an inductor and capacitor forming the core of the oscillating unit. A parallel LC resonator consists of an inductor and capacitor in parallel with each other. In reality, an LC network is never perfect and has loss in it due to the resistance in the traces connecting the parts together, and the inherent resistance 
existing in components themselves due to the material they are made from. An example of an $\mathrm{LC}$ resonator is shown in Figure 17.

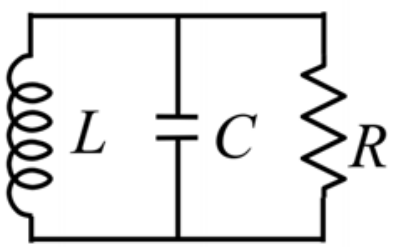

Figure 17: A parallel LC resonator consisting of a capacitor, inductor, and a resistor in parallel. The resistor represents the resistive loss in the network [35].

If an impulse is applied to the circuit in Figure 17, it will start oscillating and decay out as shown in Figure 18.

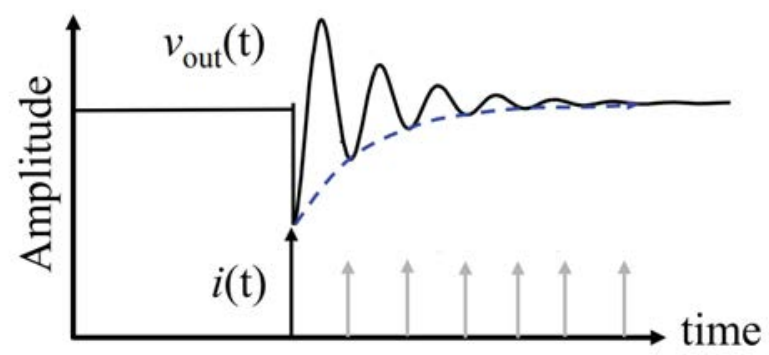

Figure 18: LC oscillator dampens and decays after an impulse signal is applied to it [35].

The response in Figure 18 is governed by:

$$
v_{\text {out }}(t)=\frac{I_{\text {pulse }} e^{-\frac{t}{2 R C}}}{C} \cos \sqrt{\frac{1}{L C}-\frac{1}{4 R^{2} C^{2}}}
$$

Observe from Eq. 2 that the system is periodic and sinusoidal due to the cosine term however it is also decaying due to the negative exponential term. The oscillation frequency can be given by:

$$
\omega_{o s c}=\sqrt{\frac{1}{L C}-\frac{1}{4 R^{2} C^{2}}}
$$

As $|R|$ is decreased in Eq. 3, the frequency of oscillation also decreases. If however $|R|$ is high and $|R|>>\sqrt{L C}$, then the frequency of oscillation can be given by:

$$
\omega_{o s c}=\sqrt{\frac{1}{L C}}
$$

A feedback loop that adds energy to the system is required to sustain the oscillation for an 
indefinite period of time, this can be achieved by adding negative resistance to the resonator core that can offset the loss in the LC tank, as shown in Figure 19.

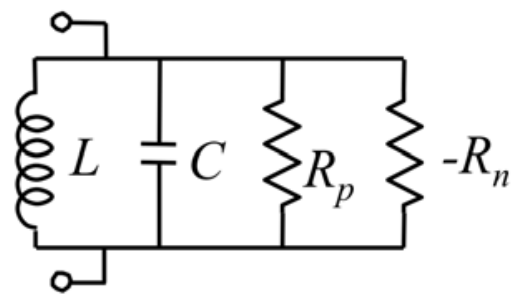

Figure 19: Negative resistance $-R_{n}$ added to the LC tank to offset the energy loss due to the tank resistance $R_{p}[35]$.

\subsubsection{Implementation of negative resistance}

There are several ways to add negative resistance to the oscillator tank via feedback loop. The three most common ways to achieve the feedback is shown in Figure 20.

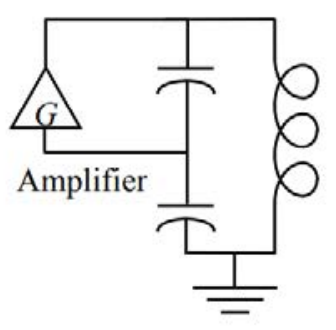

Colpitts Oscillator

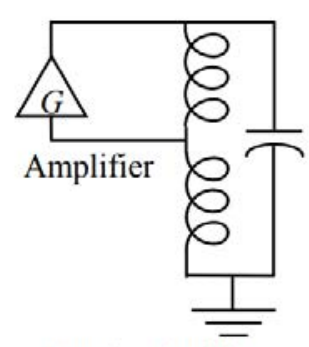

Hartley Oscillator

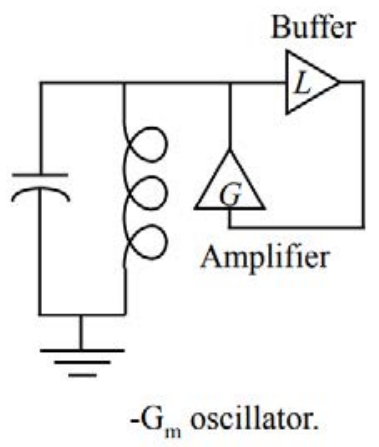

Figure 20: Three popular ways in implementing negative resistance: a) Colpitts oscillator, b) Hartley oscillator, and c) $-g_{m}$ oscillator [35].

The Colpitt oscillator uses a tapped capacitor and an amplifier to form the feedback loop, the Hartley oscillator uses a tapped inductor and an amplifier to form the feedback loop, and the negative gm $(-g m)$ oscillator uses two amplifiers to form the positive feedback loop [35]. Since negative gm oscillators are excellent when it comes to phase noise (PN) performance and are easy to implement in complementary metal oxide semiconductor (CMOS) technology, the negative gm oscillators will be discussed further.

\subsubsection{Analysis of $-g_{m}$ oscillator}

A negative gm oscillator can be implemented using two transistors as shown in Figure 21. The bipolar junction transistors (BJTs) in Figure 21 can be replaced by 
metal-oxide-semiconductor field-effect-transistors (MOSFETs) for use in CMOS technology.

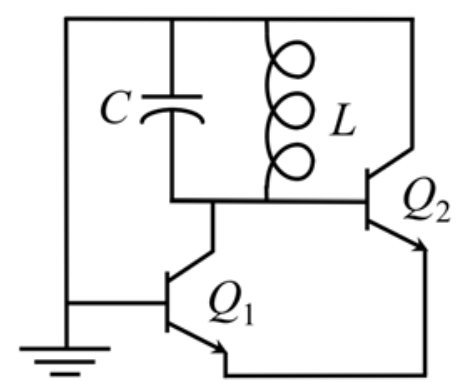

Figure 21: Implementation of negative gm oscillator using two transistors [35].

The small signal equivalent model of the circuit in Figure 21 is shown in Figure 22; where $g_{m}$ is the transconductance of the transistors, $r_{e}$ is the emitter (or source) resistance of the transistor, and $v_{\pi}$ is the base-emitter (or gate-source) voltage of the transistors.

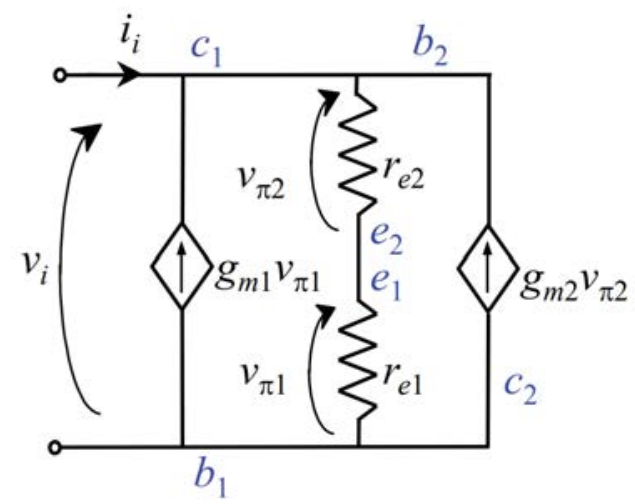

Figure 22: Small signal model of the circuit in Figure 21 [35].

Current flow analysis of the model in Figure 21 shows that:

$$
i_{i}=\frac{v_{i}}{r_{e 1}+r_{e 2}}-g_{m 1} v_{\pi 1}-g_{m 2} v_{\pi 2}
$$

If both transistors in Figure 22 are identical, then $g_{m 1}=g_{m 2}=g_{m}, v_{\pi 1}=v_{\pi 2}=v_{\pi}$, and $r_{e 1}=r_{e 2}=r_{e}$; then Eq. 5 reduces to:

$$
i_{i}=\frac{v_{i}}{2 r_{e}}-2 g_{m} v_{\pi}
$$

If the assumption is made that $g_{m} \approx \frac{1}{r_{e}}$, then Eq. 6 reduces to:

$$
i_{i}=\frac{v_{i} g_{m}}{2}-2 g_{m} v_{\pi}
$$




$$
i_{i}=\frac{v_{i} g_{m}}{2}-g_{m} v_{i}=v_{i}\left(\frac{g_{m}}{2}-g_{m}\right)=v_{i}\left(\frac{-g_{m}}{2}\right)
$$

Solving for the impedance $\left(Z_{i}\right)$ looking in where $Z_{i}=v_{i} / i_{i}$, then:

$$
Z_{i}=-R_{n}=\frac{-2}{g_{m}}
$$

For oscillation to occur, the total parallel resistance of the tank in Figure 19 must be negative (or less than zero):

$$
\left(\frac{1}{R_{p}}+\frac{1}{-R_{n}}\right)^{-1}=\left(\frac{1}{R_{p}}+\frac{-g_{m}}{2}\right)^{-1}<0
$$

Then solving for $g_{m}$ with respect to $R_{p}$ :

$$
\begin{gathered}
\left(\frac{2-g_{m} R_{p}}{2 R_{p}}\right)^{-1}<0 \Rightarrow\left(\frac{2-g_{m} R_{p}}{2 R_{p}}\right)<0 \Rightarrow\left(\frac{2}{2 R_{p}}-\frac{g_{m} R_{p}}{2 R_{p}}\right)<0 \\
\frac{1}{R_{p}}<\frac{g_{m}}{2} \quad \therefore \\
g_{m}>\frac{2}{R_{p}}
\end{gathered}
$$

Therefore the transconductance $g_{m}$ of the transistors must be greater than $2 / R_{p}$ for oscillation to occur if a negative gm configuration of the like shown in Figure 21 is used.

\subsubsection{Biasing of $-g_{m}$ oscillator}

As stated previously in Chapter 3.2.2, a negative gm oscillator can be administered using two transistors; however for proper function to occur, the circuit requires DC biasing. A few design examples of how the oscillator circuit can be implemented and biased is shown in Figure 23. 


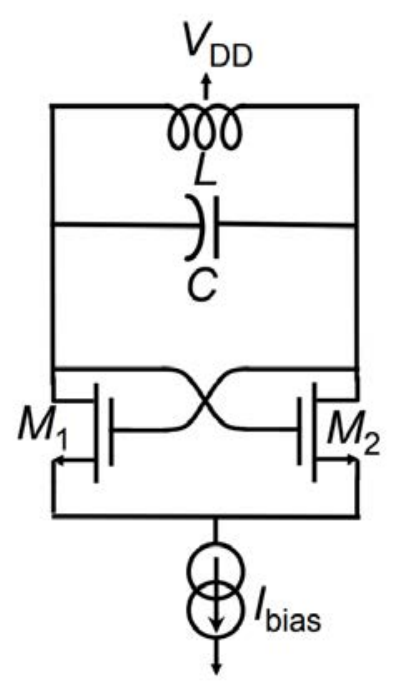

A)

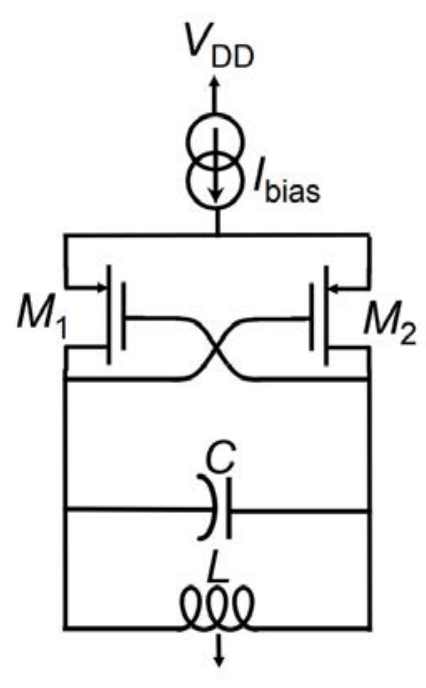

B)

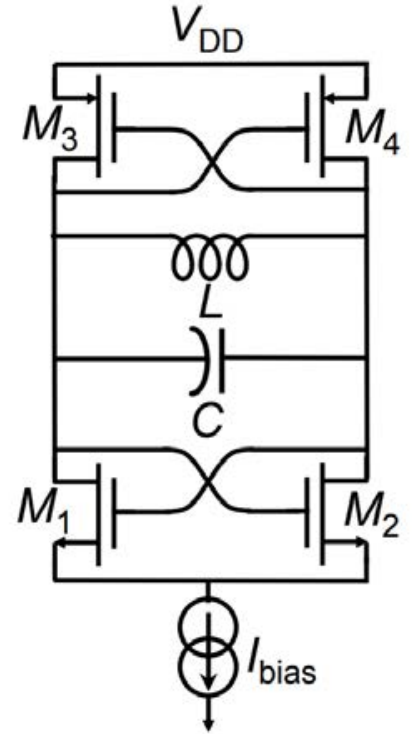

c)

Figure 23: Different ways of implementing a negative gm circuit using two MOSFETs. Figure A) uses two NMOS transistors, Figure B) uses two PMOS transistors, and Figure C) uses both two NMOS and two PMOS transistors [35].

In Figure 23, circuit A) uses two n-type metal-oxide-semiconductor(NMOS) transistors. The advantage of using NMOS transistors is that they have higher frequency response then their counterpart p-type metal-oxide-semiconductor (PMOS) transistors shown in circuit B). PMOS transistors however have a lower flicker noise ( $1 / \mathrm{f}$ or pink noise) leading to better overall phase noise $(\mathrm{PN})$ performance for the oscillator. The drawback of using PMOS transistors is that PMOS transistors require more real estate on chip IC to draw the same amount of current as NMOS transistors; almost 2-3 times as much space. Circuit C) uses both NMOS and PMOS transistors, this allows the circuit to re-use the current for two sets of transistors leading to greater $g_{m}$ for the same power consumption [35]. For this thesis research, because performance is very crucial, the PMOS configuration in circuit B) will be used.

\subsection{Phase noise performance of $-g_{m}$ oscillator}

This section will briefly touch on the phase noise performance of a negative gm oscillator. Phase noise performance can be predicted to some degree using Eq. 11, however an $\Omega_{\text {dig }}$ factor will be needed in Eq. 11 to adjust for the design used in this thesis (as the oscillator designed in the thesis does not have the same architecture as a typical negative gm differential pair voltage-controlled oscillator.) 
According to Leeson's formula [33] - [35], the phase noise of the oscillator with respect to the carrier frequency can be predicted by:

$$
P N=\left(\frac{A \omega_{o}}{2 Q \Delta \omega}\right)^{2}\left(\frac{F k T}{2 P_{s}}\right)
$$

Where $A$ takes into account the non-linear noise due to the transistor's self-cross mixing and can be approximated by $A \approx \sqrt{2} . T$ is the temperature in Kelvin and $k$ is the Boltzmann constant where $k \simeq 1.381 \times 10^{-23} \frac{\mathrm{m}^{2} \mathrm{~kg}}{\mathrm{~s}^{2} \mathrm{~K}}$. $Q$ is the quality factor of the tank where $L$ is the effective inductance of the tank and $C$ is the effective capacitance of the tank and:

$$
Q=\frac{R_{p}}{\omega_{o} L}=R_{p} \omega_{o} C
$$

$\omega_{o}$ is the center frequency of the oscillator where:

$$
\omega_{o}=\sqrt{\frac{1}{L C}}
$$

$\Delta \omega$ is the frequency offset from the center frequency at which the phase noise is desired. $P_{s}$ is the output tank power where:

$$
P_{s}=\frac{v_{\operatorname{tank}}^{2}}{2 R_{p}}
$$

$F$ is a factor due to excess noise injected to the oscillator by other sources. For short channel nm technology, $F$ can be given by:

$$
F=1+\gamma
$$

where $\gamma$ is the long channel approximation and can be approximated by $\gamma \approx 0.67$. For longer channel CMOS tech, $F$ would be:

$$
F=1+4 \gamma g_{m} R_{p}(1-\rho)
$$

where $\rho$ is the amount of time the transistors are completely switched [35]. For a digitally controlled oscillator, there would be a digital noise factor $\Lambda_{d i g}$ that would need to be accounted for such that:

$$
P N_{\mathrm{dig}}[\mathrm{dB}]=10 \cdot \log _{10}\left[\left(\frac{A \omega_{o}}{2 Q \Delta \omega}\right)^{2}\left(\frac{F k T}{2 P_{s}}\right)\right]+\Lambda_{\mathrm{dig}}[\mathrm{dB}]
$$


where:

$$
\Lambda_{\mathrm{dig}}[\mathrm{dB}]=\sum_{i=1}^{N} \Lambda_{i}[\mathrm{~dB}]=\Lambda_{1}[\mathrm{~dB}]+\Lambda_{2}[\mathrm{~dB}]+\Lambda_{3}[\mathrm{~dB}]+\cdots+\Lambda_{N}[\mathrm{~dB}]
$$

$\Lambda_{\text {dig }}$ is a collective noise summation term of all $i^{\text {th }}$ elements. These noise elements can arise due to factors such as the existence of digital quantization noise, noise susceptibility of the technology, digital noise bleeding, and also because $n_{\text {bit }}$ switching cannot be simply approximated using the narrow band frequency modulation (FM) assumption (rather the signals being fed to the core are digital square waves).

\subsection{Figure of Merit (FoM)}

The Figure of Merit (FoM) is a metric that weighs in both the phase noise performance of the oscillator and the power consumed by it and gives a production score based on the formula:

$$
\mathrm{FoM}=\mathrm{PN}\left\{f_{o}\right\}-20 \cdot \log _{10}\left(\frac{f_{o}}{f_{\text {offset }}}\right)+10 \cdot \log _{10}\left(\frac{\mathrm{P}_{D C}}{1 \mathrm{~mW}}\right)
$$

The above formula can be used to compare (and give insight) on the performance of the DCO (that will be introduced later in the thesis); and see how it measures up to old traditional VCOs.

Several offspring variations of the above formula have sprung for various architectures or situations such as $\mathrm{FoM}_{\mathrm{T}}$ and $\mathrm{FoM}_{\mathrm{L}}$; however if caution is not taken when interpreting the results, the FoM merit can be a deceptive caliber for performance.

Now that the theory of oscillation has been established, the next chapter will look at how to linearize a digitally controlled oscillator using a new perspective.

\subsection{Conclusion}

This chapter discussed the theory of oscillators; more specifically negative gm oscillators. It also discussed the oscillation start-up requirements, biasing of negative gm oscillators, Leeson's phase noise formula, and how to calculate the figure of merit for a weighted phase noise and power performance. 


\section{CHAPTER 4: LINEAR THEOREM \& DESIGN}

This section will look at the theory on how to design a linearized digitally controlled oscillator.

\subsection{Inherent Nature of LC Oscillators}

As previously stated in Chapter 3.2, the oscillation frequency of an LC oscillator can be given by:

$$
\omega_{o s c}=\sqrt{\frac{1}{L C}}
$$

More specifically in the time domain, the frequency of oscillation is given by:

$$
f=\frac{1}{2 \pi \sqrt{L C}}
$$

where L includes the combined total inductance (including parasitic) and $\mathrm{C}$ includes the combined total capacitance of the oscillator topology. There are only two first degree variables that can control the output frequency according to Eq. (20), namely the inductance and the capacitance. On-chip inductors are usually difficult to implement on $\mathrm{Si}$ technology. Inductors take up large amount of real estate and they are very lossy with poor

Q [40]; for these reasons on-chip inductors tend to be fixed. The more practical approach is to make the capacitance variable for tuning the frequency. If the inductance is assumed to be approximately the same over a certain frequency span, then Eq. (20) can be reduced to:

$$
f=\frac{1}{2 \pi \sqrt{L C}}=\left(\frac{1}{2 \pi \sqrt{L}}\right)\left(\frac{1}{\sqrt{C}}\right)=\frac{\delta}{\sqrt{C}}
$$

where

$$
\delta=\frac{1}{2 \pi \sqrt{L}} \approx \text { constant }
$$

Before touching on the fact that on chip variable capacitors are usually non-linear [16] (whether they are varactors or CMOS based, or whether they are current controlled, voltage controlled, or controlled by some other means), the inherent nature of the function $f(C)=$ $\delta / \sqrt{C}$ itself is non-linear to begin with. The non-linear behavior of the function $\delta / \sqrt{C}$ must be dealt with first before taking on the issue of non-linear capacitance. Some examples of the shape of a $\delta / \sqrt{C}$ curve is shown in Figure 24 . 


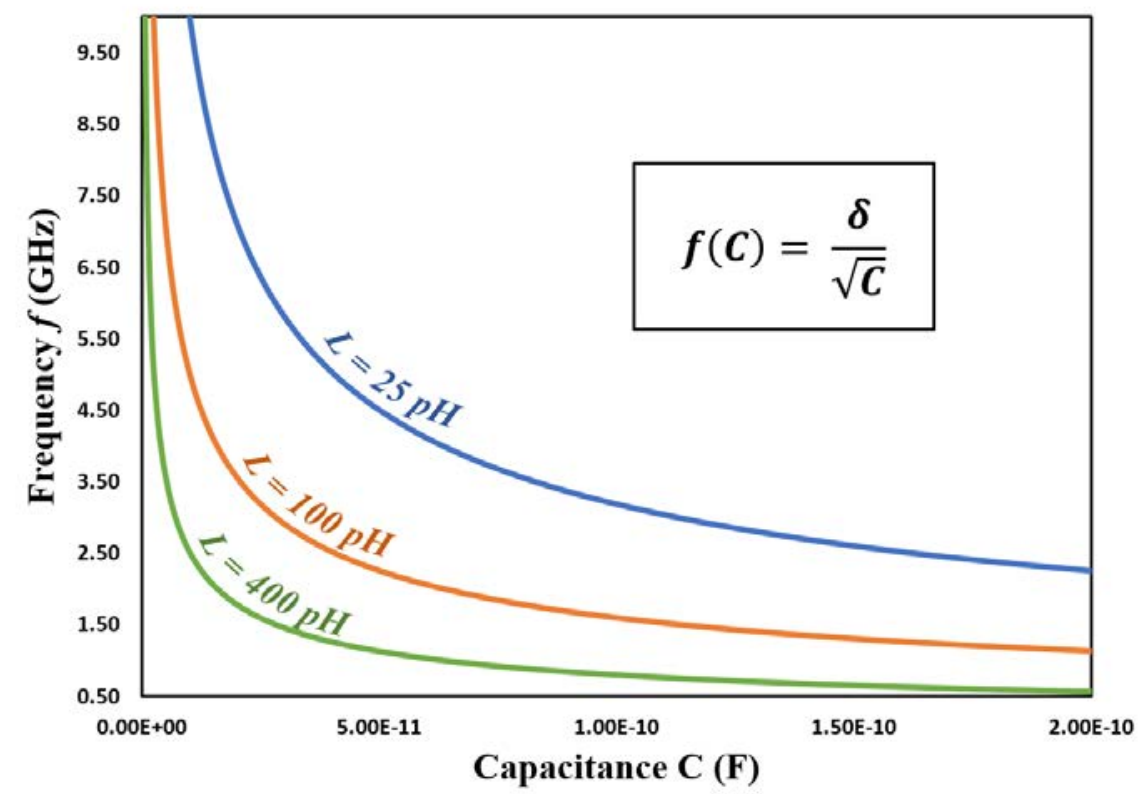

Figure 24: Typical LC curve shape of $\mathrm{L}=25 \mathrm{pH}, 100 \mathrm{pH}$, and $400 \mathrm{pH}$.

As is evident from Figure 24, the curve is nonlinear; however graphically certain portions of the curve look linear to the unaided eye. What if a portion of the curve over a subset $\left[C_{l}, C_{u}\right]$ (where $C_{l}$ is the lower bound and $C_{u}$ is the upper bound) in Figure 24 can be approximated very closely as a linear equation $y=m x+b$ such that:

$$
f=\frac{\delta}{\sqrt{C}} \approx m C+b
$$

A statistical approach to determining if portions of the curve can be treated as linear approximations is deduced in the next section.

\subsection{Statistical Analysis of Linear Approximation}

Regression is the study of dependence, and linear regression is used as a predictor of how the linearity of one response is influenced by another [41]. The coefficient of determination $\left(\mathrm{R}^{2}\right)$ is a scale free summary of the strength between the dependent and independent variable, it is a measure of how close the data is fitted to the regression line [41]. Caution must be taken when using $\mathrm{R}^{2}$ and while $\mathrm{R}^{2}$ is not always used as the best predictor for fit (examples being noisy data, nonlinear data, and outliers), for a small continuous subset of the curve in Figure $24, \mathrm{R}^{2}$ can be used as an excellent figure of merit to define the approximate strength of a linear relationship. The closer $\mathrm{R}^{2}$ is to 1 , the more the two variables can be assumed 
to be linearly correlated with each other in a linear fit. Linear regression for n-points is given by:

$$
y=m x+b
$$

where $x$ is the independent variable and $y$ is the dependent variable. The slope $m$ and intercept $b$ is defined as:

$$
\begin{array}{r}
m=\frac{n\left(\sum_{i=1}^{n} x_{i} y_{i}\right)-\left(\sum_{i=1}^{n} x_{i}\right)\left(\sum_{i=1}^{n} y_{i}\right)}{n\left(\sum_{i=1}^{n} x_{i}^{2}\right)-\left(\sum_{i=1}^{n} x_{i}\right)^{2}} \\
b=\frac{\left(\sum_{i=1}^{n} y_{i}\right)\left(\sum_{i=1}^{n} x_{i}^{2}\right)-\left(\sum_{i=1}^{n} x_{i}\right)\left(\sum_{i=1}^{n} x_{i} y_{i}\right)}{n\left(\sum_{i=1}^{n} x_{i}^{2}\right)-\left(\sum_{i=1}^{n} x_{i}\right)^{2}}
\end{array}
$$

To fit a linear regression to a subset $\left[C_{l}, C_{u}\right]$ of the continuous curve in Figure 24, an integral transformation using first principles must be made to Eq. (25) and Eq. (26) such that:

$$
m=\lim _{n \rightarrow \infty} \frac{n \Delta C\left(\delta \sum_{i=1}^{n} \sqrt{C_{i}} \Delta C\right)-\left(\sum_{i=1}^{n} C_{i} \Delta C\right)\left(\delta \sum_{i=1}^{n} \frac{\Delta C}{\sqrt{C}}\right)}{n \Delta C\left(\sum_{i=1}^{n} C_{i}^{2} \Delta C\right)-\left(\sum_{i=1}^{n} C_{i} \Delta C\right)^{2}}
$$

where:

$$
\begin{aligned}
& C_{\Delta}=C_{u}-C_{l} \\
& \Delta C=\left|\frac{C_{\Delta}}{n-1}\right|
\end{aligned}
$$

Realizing that:

$$
\begin{gathered}
\lim _{n \rightarrow \infty}\left|\frac{C_{\Delta}}{n-1}\right|=\lim _{n \rightarrow \infty}\left|\frac{C_{\Delta}}{n}\right| \\
\text { then } \lim _{n \rightarrow \infty} \sum_{i=1}^{n} \gamma_{i}(n) \Delta C=\int_{C_{l}}^{C_{u}} \gamma(C) d C
\end{gathered}
$$


Then Eq. (27) reduces to:

$$
m=\frac{\delta\left(C_{\Delta}\right)\left(\int_{C_{l}}^{C_{u}} \sqrt{C} d C\right)-\delta\left(\int_{C_{l}}^{C_{u}} C d C\right)\left(\int_{C_{l}}^{C_{u}} \frac{d C}{\sqrt{C}}\right)}{\left(C_{\Delta}\right)\left(\int_{C_{l}}^{C_{u}} C^{2} d C\right)-\left(\int_{C_{l}}^{C_{u}} C d C\right)^{2}}
$$

Solving for Eq. (27) gives:

$$
m=\frac{\frac{1}{3 \pi \sqrt{L}}\left(C_{u}-C_{l}\right)\left(C_{u}^{3 / 2}-C_{l}^{3 / 2}\right)-\frac{1}{2 \pi \sqrt{L}}\left(C_{u}^{2}-C_{l}^{2}\right)\left(\sqrt{C_{u}}-\sqrt{C_{l}}\right)}{\frac{1}{3}\left(C_{u}-C_{l}\right)\left(C_{u}^{3}-C_{l}^{3}\right)-\frac{1}{4}\left(C_{u}^{2}-C_{l}^{2}\right)^{2}}
$$

Likewise, Eq. (26) reduces to:

$$
\begin{gathered}
b=\frac{\delta\left[\left(\int_{C_{l}}^{C_{u}} \frac{d C}{\sqrt{C}}\right)\left(\int_{C_{l}}^{C_{u}} C^{2} d C\right)-\left(\int_{C_{l}}^{C_{u}} C d C\right)\left(\int_{C_{l}}^{C_{u}} \sqrt{C} d C\right)\right]}{\left(C_{\Delta}\right)\left(\int_{C_{l}}^{C_{u}} C^{2} d C\right)-\left(\int_{C_{l}}^{C_{u}} C d C\right)^{2}} \\
b=\frac{\frac{1}{3 \pi \sqrt{L}}\left(\sqrt{C_{u}}-\sqrt{C_{l}}\right)\left(C_{u}^{3}-C_{l}^{3}\right)-\frac{1}{6 \pi \sqrt{L}}\left(C_{u}^{2}-C_{l}^{2}\right)\left(C_{u}^{3 / 2}-C_{l}^{3 / 2}\right)}{\frac{1}{3}\left(C_{u}-C_{l}\right)\left(C_{u}^{3}-C_{l}^{3}\right)-\frac{1}{4}\left(C_{u}^{2}-C_{l}^{2}\right)^{2}}
\end{gathered}
$$

In a similar fashion to regression, the correlation coefficient value is defined by:

$$
r=\frac{n\left(\sum_{i=1}^{n} x_{i} y_{i}\right)-\left(\sum_{i=1}^{n} x_{i}\right)\left(\sum_{i=1}^{n} y_{i}\right)}{\sqrt{\left[n \sum_{i=1}^{n} x^{2}-\left(\sum_{i=1}^{n} x\right)^{2}\right]\left[n \sum_{i=1}^{n} y^{2}-\left(\sum_{i=1}^{n} y\right)^{2}\right]}}
$$


Taking the integral transformation of $r$ and solving for it gives:

$$
r=\frac{\delta\left(\int_{C_{l}}^{C_{u}} \sqrt{C} d C\right) \frac{\delta\left(\int_{C_{l}}^{C_{u}} C d C\right)\left(\int_{C_{l}}^{C_{u}} \frac{d C}{\sqrt{C}}\right)}{C_{\Delta}}}{\left.r\left[\int_{C_{l}}^{C^{C_{u}}} C d C\right)^{2}\right]\left[\delta_{C_{l}}^{2} d C\right)-\frac{\left.\left.\int_{C_{l}}^{C_{u}} \frac{d C}{C}\right)-\frac{\left.\delta^{2}\left(\int_{C_{l}}^{C_{u}} \frac{1}{\sqrt{C}}\right)^{2}\right]}{C_{\Delta}}\right]}{\sqrt{\left[\frac{1}{3}\left(C_{u}^{3}-C_{l}^{3}\right)\left(C_{u}-C_{l}\right)-\frac{1}{4}\left(C_{u}^{2}-C_{l}^{2}\right)^{2}\right] \beta}}}
$$

where:

$$
\beta=\left[\left|\frac{\ln \left(C_{u}\right)-\ln \left(C_{l}\right)}{4 \pi^{2} L}\right|\left(C_{u}-C_{l}\right)-\frac{\left(\sqrt{C_{u}}-\sqrt{C_{l}}\right)^{2}}{\pi^{2} L}\right]
$$

The coefficient of determination is simply then:

$$
\mathrm{R}^{2} \text { (coefficient of determintation) }=r^{2}
$$

\subsection{Using $\mathrm{R}^{2}$ to Choose Region of Linearity}

Using Eq. (36) to Eq. (38), field maps can be generated to determine regions of linearity based on the inductance, capacitance, and frequency requirements.

\subsubsection{Topographical linearity field-maps}

In Eq. (20) the dependent variable is frequency. Since frequency is usually the variable of interest, one can fix the frequency steps and then determine the required capacitance. Then one can use Eq. (36) to Eq. (38) to plot $\mathrm{R}^{2}$ curves using numerical computational software such as excel or MATLAB based on the required frequency span. Such linearity field maps for different center frequencies or different values of inductance for a frequency span of $1 \mathrm{GHz}$ and $4 \mathrm{GHz}$ are shown in Figure 25 to Figure 26 respectively. A 3D rendering of the $1 \mathrm{GHz}$ span field map using MATLAB and excel is shown in Figure 27. 
As expected, from Figure 25 and Figure 26, larger frequency spans will be less linear. The field maps also predict that it will be easier to achieve better linearity for larger frequency spans at higher frequencies. This could explain why DCOs [42], [43], and [44] managed to get relatively good linearity by sheer opportunity at such high frequencies using switch capacitor banks without much explanation as to why.

Field Map of $R^{2}$ vs Frequency

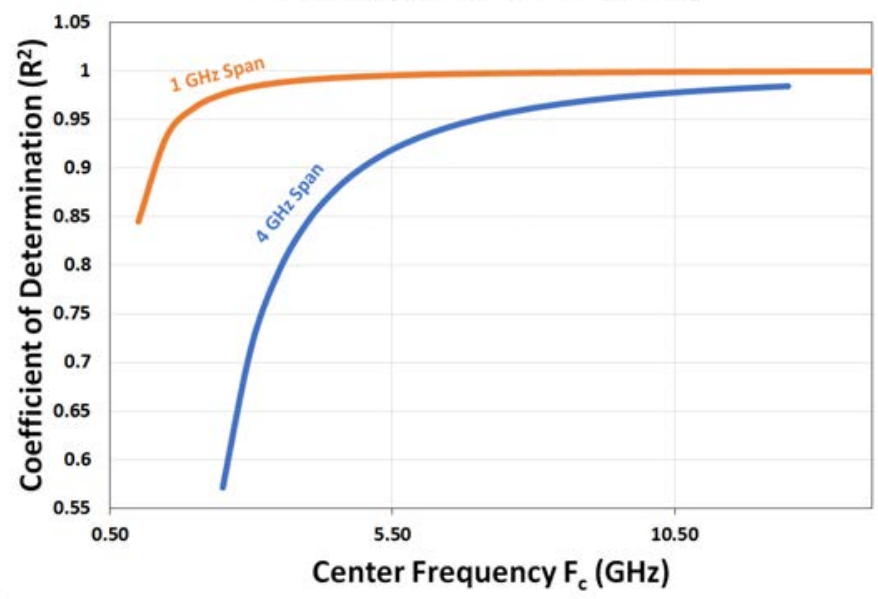

Figure 25: $\mathrm{R}^{2}$ values for different center frequency for a frequency span of $1 \mathrm{GHz}$ and 4 $\mathrm{GHz}$.

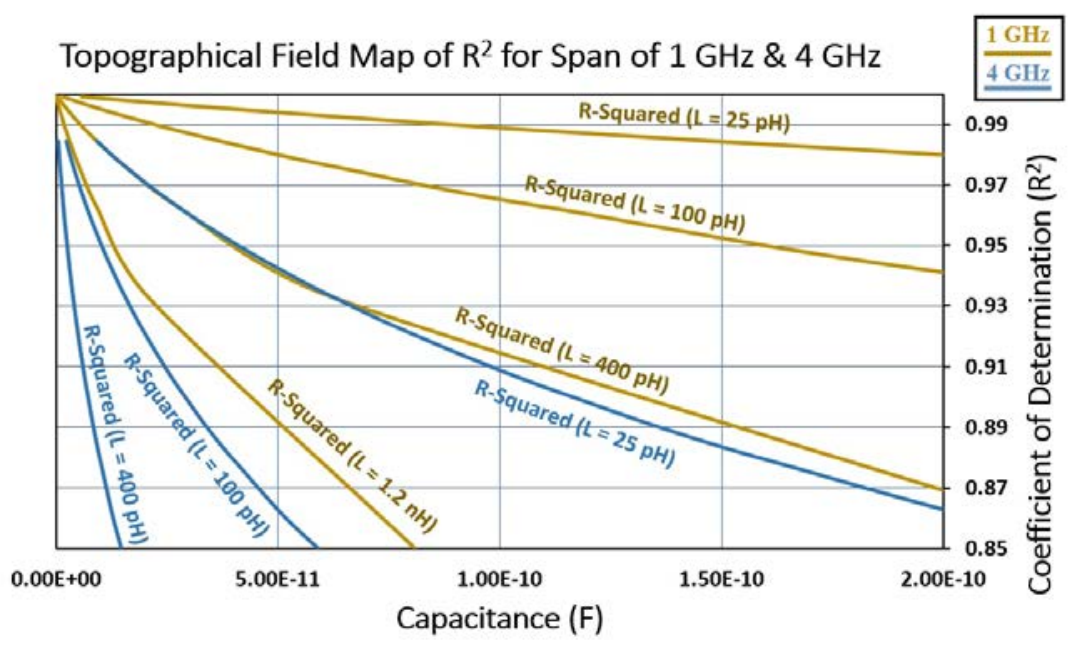

Figure 26: Topographical map of $\mathrm{R}^{2}$ for a frequency span of $1 \mathrm{GHz}$ and $4 \mathrm{GHz}$ for various inductor values. 


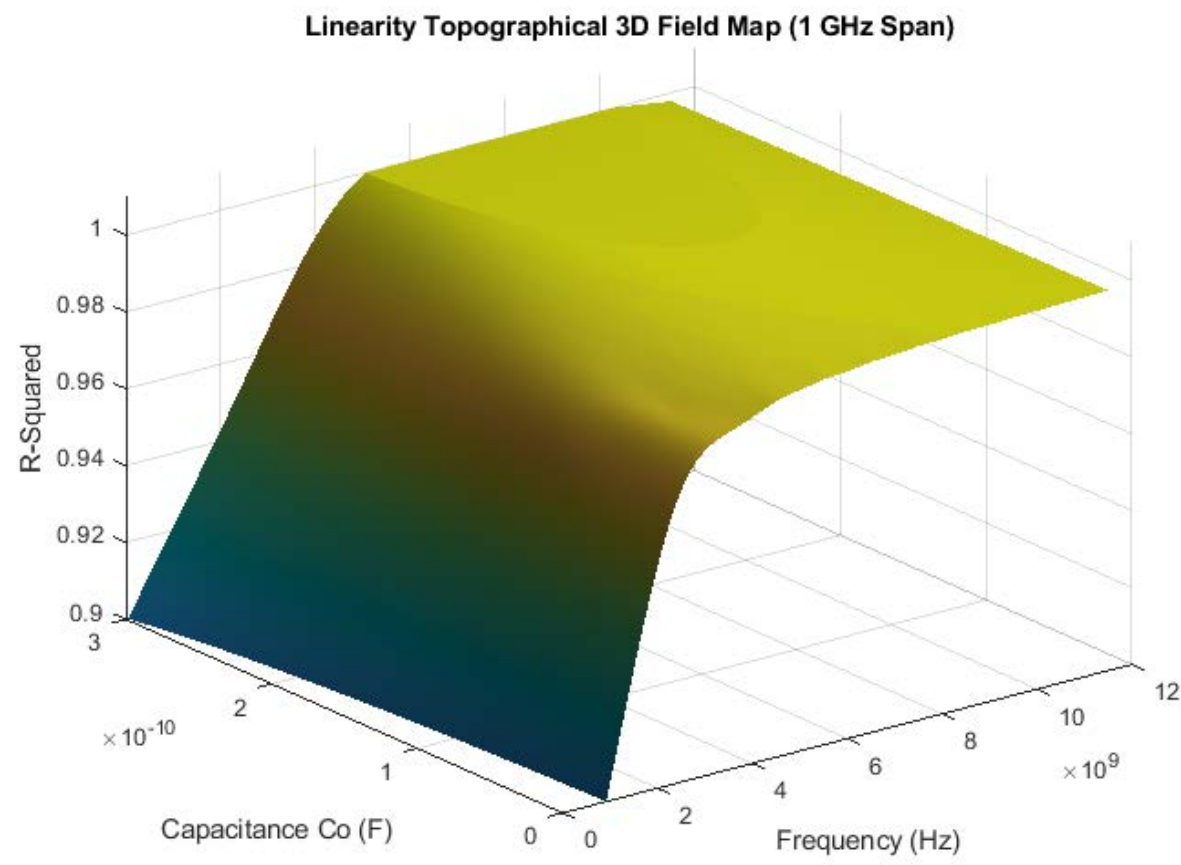

Figure 27: Linearity topographical 3D field map of frequency span of $1 \mathrm{GHz}$.

\subsection{Example of Using $\mathrm{R}^{2}$ Field Maps}

Given an inductor inductance of $\backsim 400 \mathrm{pH}$ (typical of nanometer technology kit inductor) and $70 \mathrm{pH}$ of extracted parasitic, can a $6 \mathrm{GHz}-7 \mathrm{GHz}$ oscillator be designed with good linearity by taking advantage of its natural inherent nature?

The required frequency span of the oscillator is $1 \mathrm{GHz}$ so the first step will be to plot the $\mathrm{R}^{2}$ field map of $1 \mathrm{GHz}$ span for $\mathrm{L}=470 \mathrm{pH}$. The corresponding field maps of Figure 25 and Figure 26 combined is shown in Figure 28. 
Topographical Map of $\mathrm{R}^{2}$ for Frequency Span of $1 \mathrm{GHZ}(\mathrm{L}=470 \mathrm{pH})$

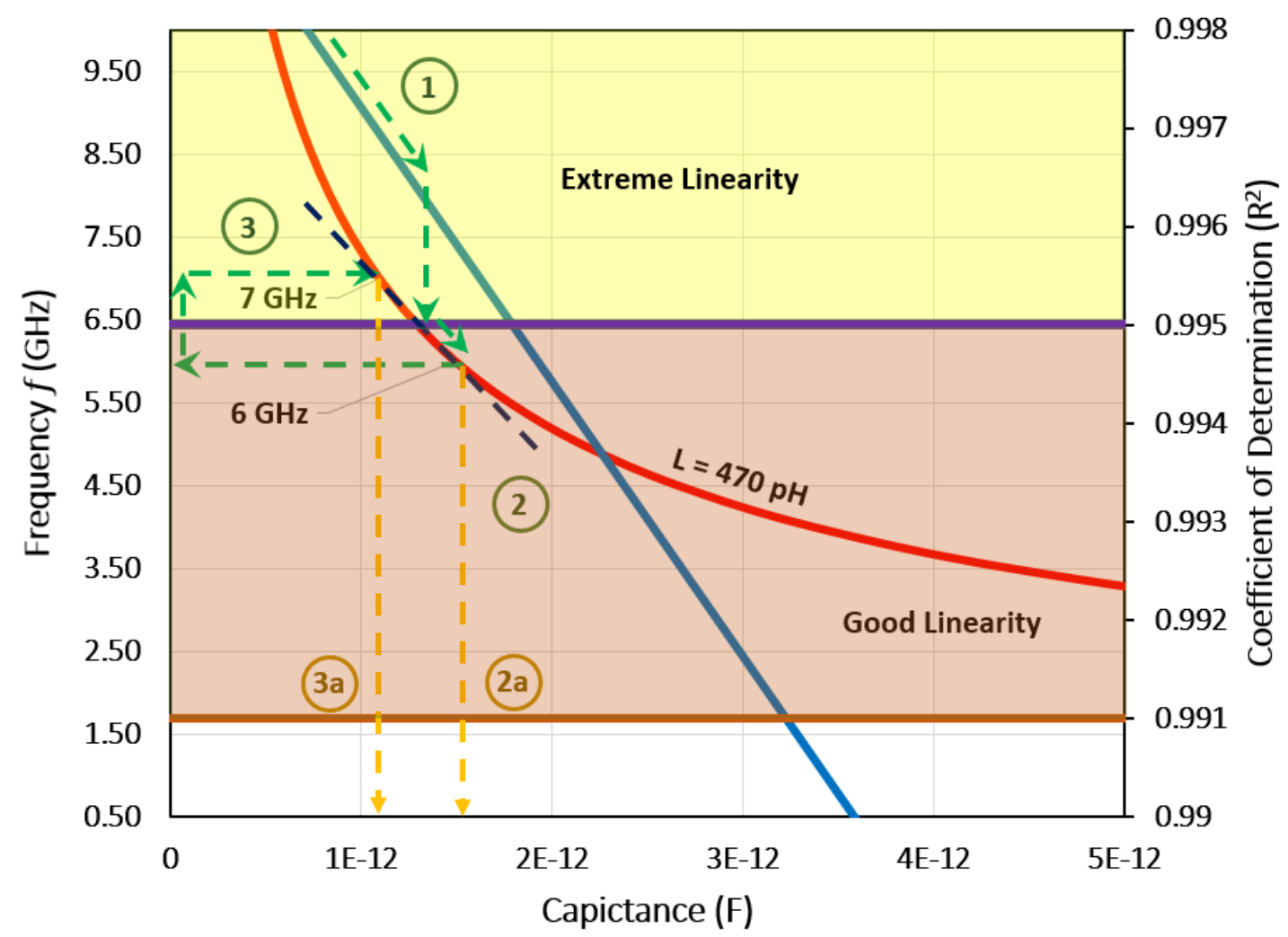

Figure 28: Field map of $\mathrm{R}^{2}$ for a frequency span of $1 \mathrm{GHz}(\mathrm{L}=470 \mathrm{pH})$. Extremely linearity is defined as an error of $0.5 \%$ or less from $100 \%$ linearity by the author. Good linearity is defined as an error of $[0.9 \%-0.5 \%)$ from $100 \%$ linearity by the author.

Looking at the $1 \mathrm{GHz}$ span field map (Figure 28) and tracing the $\mathrm{R}^{2}$ curve to $6.5 \mathrm{GHz}$ (center frequency of 6-7 GHz) by following arrow (1), it can be observed that $6.5 \mathrm{GHz}$ falls under the region of 'Extreme Linearity' (defined by the author as $\leq 0.5 \%$ from $100 \%$ linearity), therefore the map predicts that an oscillator with excellent linearity can be designed from a span of $6 \mathrm{GHz}-7 \mathrm{GHz}$. Arrows (2) and (2a) can be followed to determine the value of the capacitance at $6 \mathrm{GHz}$, and arrows (3) and (3a) can be traced to determine the capacitance required at $7 \mathrm{GHz}$.

Since the frequency between $6 \mathrm{GHz}-7 \mathrm{GHz}$ is shown to be highly linear with $\mathrm{R}^{2} \geq 0.991$, then one can state that the linear approximation is a valid assumption (based on the clause introduced by the author). Therefore, the natural LC response given by Eq. 20 can be reduced to Equation 23. For an oscillator between $6 \mathrm{GHz}$ and $7 \mathrm{GHz}$, the required capacitance is $1.5 \mathrm{pF}$ and $1.1 \mathrm{pF}$ respectively. For a 16-bit DCO, each step size will require 
a capacitance of:

$$
\text { capacitance per bit } \approx \frac{1.5 p F-1.1 p F}{16} \simeq 25 f F
$$

For a DCO whose frequency will vary depending on the bits, the following substitutions will have to be made to Eq. 30 and Eq. 32:

$$
\begin{gathered}
m_{C \rightarrow b i t} \simeq \frac{m C_{\Delta}}{B i t_{\text {max }}-B i t_{\text {min }}} \\
b_{C \rightarrow b i t}=b-\left|m C_{l}\right|+\left|m_{C \rightarrow b i t} B i t_{\text {min }}\right|
\end{gathered}
$$

where $B i t_{\max }$ is the maximum bit number and $B i t_{\min }$ is the minimum bit number. The DCO frequency can then be predicted by the simple linear equation:

$$
f \approx\left[m_{C \rightarrow b i t}\right] B i t+b_{C \rightarrow b i t}
$$

Equation (41), (30), (32), (36), (39), and (40) predicts that over the subset of $[6 \mathrm{GHz}, 7$ $\mathrm{GHz}]$ the frequency of the DCO will be:

$$
\begin{gathered}
f=-2.55 \times 10^{21}\left(\frac{\mathrm{Hz}}{\mathrm{F}}\right) C+9.78 \times 10^{9} \mathrm{~Hz} \\
f=-7.64 \times 10^{7}\left(\frac{\mathrm{Hz}}{\mathrm{Bit}}\right) B i t+7.11 \times 10^{9} \mathrm{~Hz} \\
R^{2}=0.995
\end{gathered}
$$

A plot of the DCO frequency vs DCO bit for 16 bits is shown in Fig. 29. Using excel, the trend line and coefficient of correlation is also given on the plot.

\subsection{Implementing a Physical DCO Using $\mathrm{R}^{2}$ Linearization Technique}

This section gives an example of how to make a linear DCO using the theoretical example from the previous chapter.

\subsubsection{LC oscillator core}

A negative gm pair architecture with PMOS transistors like the one discussed in Chapter 3.2.3 can be used for the oscillator; more specifically a core like the one shown in Figure 30. PMOS negative gm pairs are relatively simple to implement, they have good phase noise 


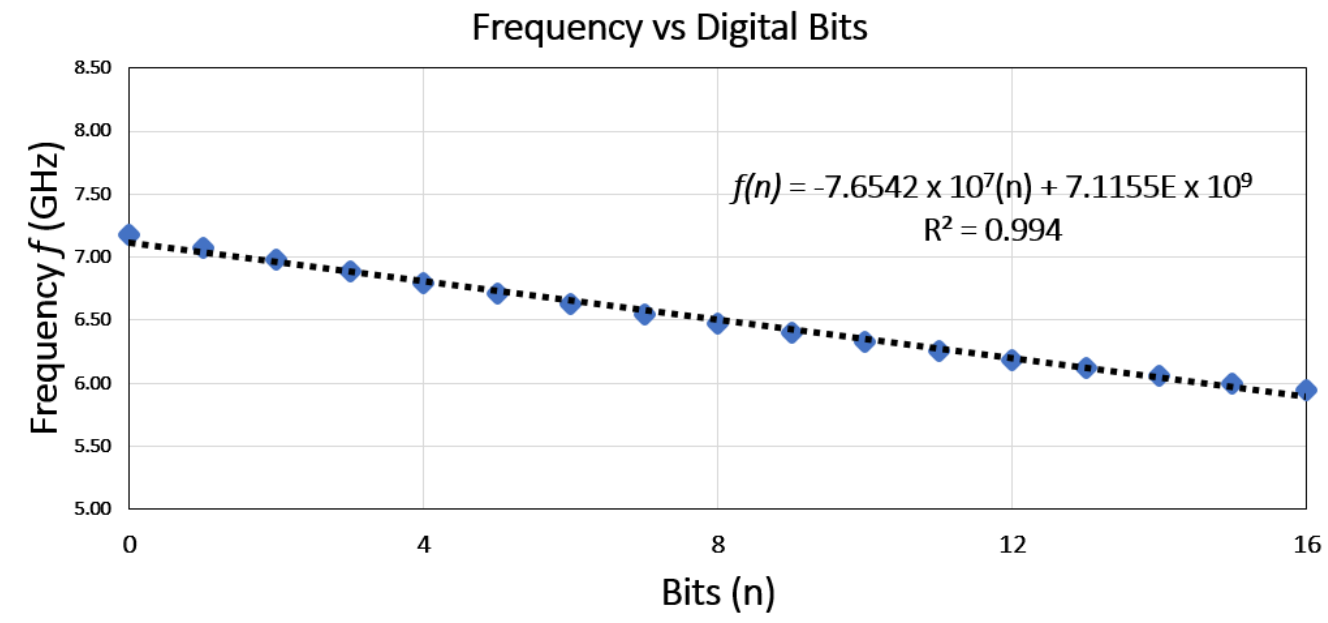

Figure 29: Frequency vs bits of the DCO designed from Fig. 28.

performance, and they require minimal components.

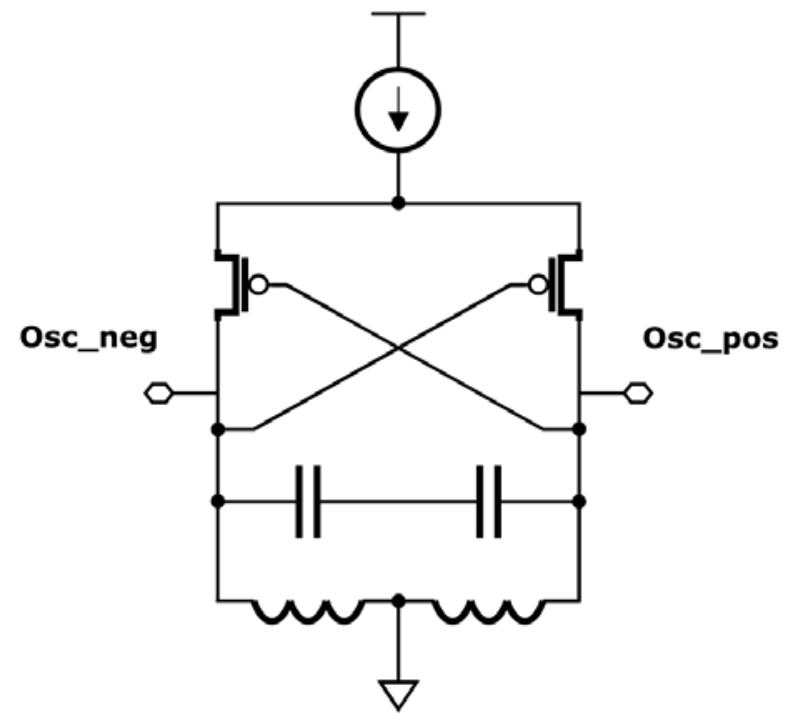

Figure 30: A PMOS negative gm pair topology.

\subsubsection{Capacitor banks for equal frequency step sizes}

Varactors are non-linear and posses the voltage vs capacitance curve shown in Figure 31. For those that want to design a multi-bank VCO using the linearity field map technique, only the linear portion of the voltage vs capacitance curve can be used (Figure 31). For a DCO, equal capacitance bank sets of MIM, MOM, moscaps, or high Q fixed varactors can be used to switch through the frequency steps. An example of a bank network is shown in Figure 32 . 


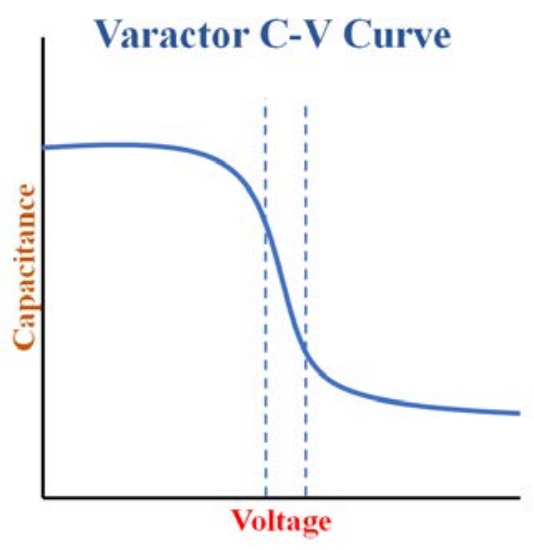

Figure 31: Capacitance vs voltage curve of a typical varactor.

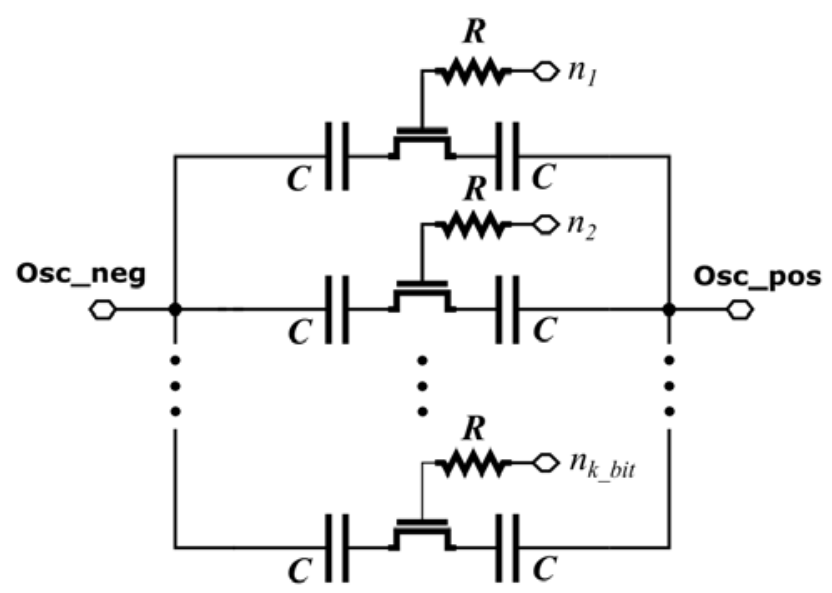

Figure 32: All capacitors have fixed capacitance for equal frequency steps.

The required difference between the capacitance of the bank when it is on $\left(C_{\max }\right)$ and when it is off $\left(C_{\min }\right)$ is given by:

$$
C_{\max }-C_{\min }=\frac{1}{4 \pi^{2} L b_{\text {tno }}}\left(\frac{1}{f_{\min }^{2}}-\frac{1}{f_{\max }^{2}}\right)
$$

where $b_{t n o}$ is the total number of bits, and $C_{T}$ is the total tank capacitance (including parasitic capacitance such as $C_{g d}$ and $C_{g s}$ of the differential pair and buffer). For a $6 \mathrm{GHz}$ - $7 \mathrm{GHz}$ oscillator with a 16-bit step, total tank inductance of $470 \mathrm{pH}$, and total capacitance of $0.7 \mathrm{pF}$ then:

$$
C_{\text {max }}-C_{\text {min }} \simeq 25 f F
$$

The on/off capacitance ratio of the bank can be given by: 


$$
\frac{C_{\max }}{C_{\min }}=\left(\frac{1-C_{T} f_{\min }^{2} 4 \pi^{2} L}{1-C_{T} f_{\max }^{2} 4 \pi^{2} L}\right)\left(\frac{f_{\max }^{2}}{f_{\min }^{2}}\right)
$$

For a total tank capacitance of $0.7 \mathrm{pF}, 470 \mathrm{pH}$ inductance, and $16 \mathrm{bit}$, then the on/off bank ratio for a $6 \mathrm{GHz}-7 \mathrm{GHz}$ oscillator is 2 . Each bit $n_{k \_b i t}$ in Figure 32 is digitally controlled by a digital circuit either pulled up to VDD high (digital bit 1) or pulled down to ground (GND) low (digital bit 0).

The overall design of the bank (including the transistor switch) will be non-linear and the oscillator voltage swing will be large at the two nodes of the bank, however for simplicity the entire bank can be treated as a black box where the time average on/off total capacitance of the bank (including parasitic from the switch transistor) is of importance for the design of the DCO. The same non-linearity and non-linear elements will exist from bank to bank (if the bank designs are identical) giving a linear step-through (from bank to bank switch) of frequency.

\subsubsection{Banks on source side of negative gm transistors}

The smallest capacitor available (and hence the smallest capacitance available) can be limited by the technology used to implement the oscillator. If two current mirrors are used to sink or source the oscillator (one at each source node of each negative gm transistor), then banks (like those in Figure 32) can also be implemented on the source side of the negative gm transistors. The resulting effect would be that the capacitance of the banks on the source side would translate to the drain side of the transistors (and hence adding capacitance to the overall tank capacitance) by a factor given by [36] - [39]:

$$
C_{e q}=-C_{b a n k}\left[\frac{g_{m}^{2}}{g_{m}^{2}+4\left(C_{b a n k}^{2} \omega_{o s c}^{2}\right)}\right]
$$

Looking at Eq. 44, the shrink factor is less than unity $(<1)$. This would allow finer frequency tuning control of the oscillator since the capacitance on the source is effectively shrunk when seen from the drain side.

Note that the transformation of Eq. 44 is that of a small signal transformation, and the DCO operates in large signal, however it can still be useful for designing the DCO (as it can be used for approximation). 


\subsection{Tuning Range}

The tuning range (TR) of an oscillator is a description of how large the frequency span of an oscillator is with respect to its center frequency. The tuning range is given in percentage and is defined by:

$$
\text { Tunning Range (TR) } \%=100 \cdot\left(\frac{f_{\max }-f_{\min }}{f_{c}}\right)=100 \cdot\left(\frac{2 f_{\max }-2 f_{\min }}{f_{\max }+f_{\min }}\right)
$$

The tuning range for an oscillator that has a frequency span of 7-6 GHz would be:

$$
100 \cdot\left(\frac{(2) 7 \mathrm{GHz}-(2) 6 \mathrm{GHz}}{7 \mathrm{GHz}+6 \mathrm{GHz}}\right) \simeq 15.4 \%
$$

\subsubsection{Relationship between tuning range and linearity}

The tuning range is closely related to linearity of the oscillator (if equal frequency step sizes is used to step through the frequency of the oscillator), in fact the tuning range is proportional to the linearity:

Tunning Range $(\mathrm{TR}) \propto R^{2}$

And the induction proof of that (though not exhaustive) is very simple:

\section{Tunning Range $\propto$ frequency}

\section{2. frequency $\propto$ capacitance}

3. capacitance $\propto R^{2}$

$\therefore$ Tuning Range $\propto R^{2}$

Table 1 below shows four different cases of the same tuning range with different factors such as frequency, inductance, and capacitance being changed; however the resulting linearity is always the same (though the proof for such statement has not been given and is out of the scope of the thesis). 
TABLE 1: Tuning Range vs Linearity for Four Different Test Cases.

\begin{tabular}{|c|c|c|c|}
\hline TR & Center Frequency $f_{c}$ & $\mathbf{L}$ & $\mathbf{R}^{\mathbf{2}}$ \\
\hline $15 \%$ & $6.5 \mathrm{GHz}$ & $500 \mathrm{pH}$ & 0.997 \\
\hline $15 \%$ & $6.5 \mathrm{GHz}$ & $300 \mathrm{pH}$ & 0.997 \\
\hline $15 \%$ & $3.5 \mathrm{GHz}$ & $500 \mathrm{pH}$ & 0.997 \\
\hline $15 \%$ & $200 \mathrm{MHz}$ & $1 \mathrm{nH}$ & 0.997 \\
\hline
\end{tabular}

If $f_{u}$ is the upper frequency of the tuning range, $f_{l}$ is the lower frequency, and $f_{c}$ is the center frequency, then:

$$
\begin{aligned}
& f_{u}=f_{c}\left(1+\frac{\mathrm{TR} \%}{200}\right) \\
& f_{l}=f_{c}\left(1-\frac{\mathrm{TR} \%}{200}\right)
\end{aligned}
$$

And:

$$
\begin{aligned}
& C_{u}=\frac{1}{4 \pi^{2} f_{c}^{2}\left(1-\frac{\mathrm{TR} \%}{200}\right)^{2} L} \\
& C_{l}=\frac{1}{4 \pi^{2} f_{c}^{2}\left(1+\frac{\mathrm{TR} \%}{200}\right)^{2} L}
\end{aligned}
$$

The rest then follows by substituting the new expressions for $C_{u}$ and $C_{l}$ in Eq. 36 - Eq. 38 to get the relationship between linearity and tuning range (left as an exercise for the reader).

It is also expected that the higher the tuning range, the lower the linearity will be since the frequency span will be larger. An example of the relationship between linearity and tuning range for a center frequency $f_{c}$ of $6.5 \mathrm{GHz}$ and $\mathrm{L}=500 \mathrm{pH}$ is shown in Figure 33. From the graph it can be seen that a tuning range of approximately $25 \%$ corresponds to a linearity of $\mathrm{R}^{2}=0.99$. A $3 \mathrm{D}$ pictorial relationship between linearity and tuning range is shown in Figure 34 . 


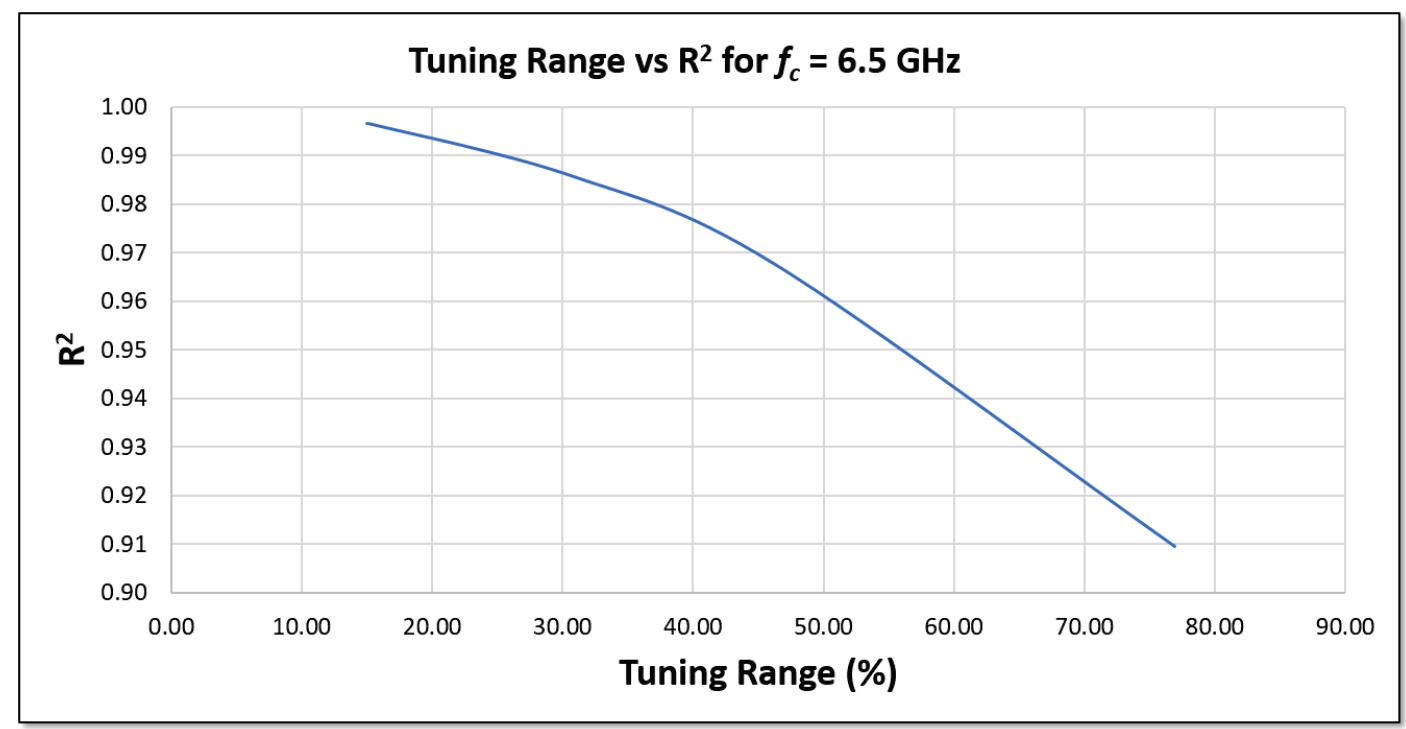

Figure 33: Relationship between linearity and tuning range.

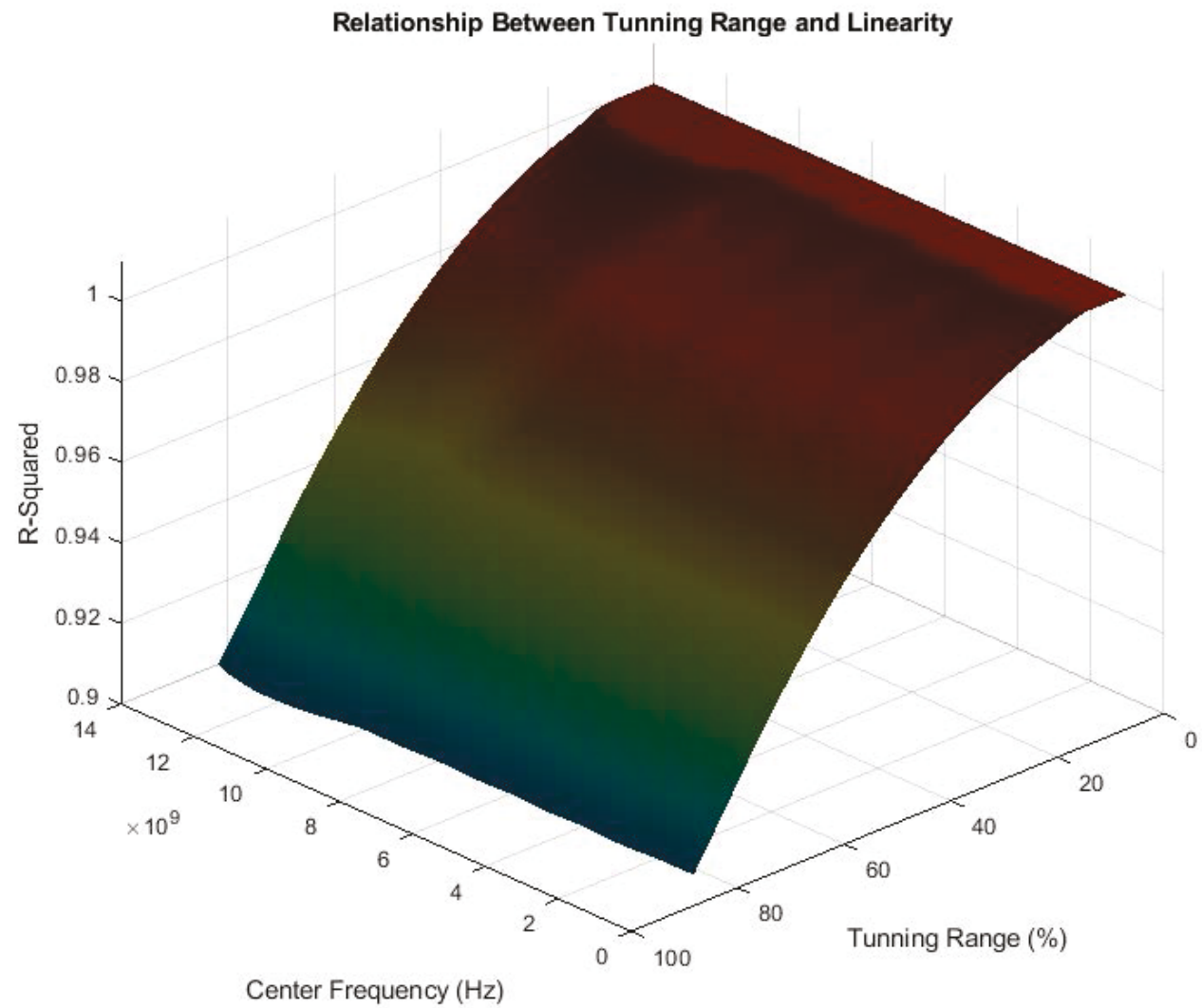

Figure 34: Relationship between linearity and tuning range from 3D perspective.

As expected the linearity is constant for the same tuning range regardless of center frequency. 


\subsection{Conclusion}

This Chapter discussed the theory behind linearizing a digitally controlled oscillator. It discussed the inherent nature of LC oscillators, it provided a statistical method for linear approximation, it introduced a new concept know as topographical linearity field maps, it gave an example on how to use the linearity field maps, and then designed a theoretical DCO using the linearity field maps. The Chapter also discussed about tuning range and the relationship between linearity and tunning range. 


\section{CHAPTER 5: SCHEMATIC DESIGN}

This chapter will look at a design of a $6 \mathrm{GHz}$ to $7 \mathrm{GHz}$ digitally controlled oscillator designed in Cadence Virtuoso. The schematic was implemented using $40 \mathrm{~nm}$ Taiwan Semiconductor Manufacturing Company (TSMC) technology kit that contained $1.1 \mathrm{~V}$ transistors, $2.5 \mathrm{~V}$ transistors, and $3.3 \mathrm{~V}$ transistors.

***Note: All circuits presented in this Chapter were either designed by the author from scratch or ported from a generic schematic and re-designed.

\subsection{Schematic Design of DCO Core and DCO Core Buffers}

The schematic design of the core of the digital controlled oscillator is shown in Figure 35.

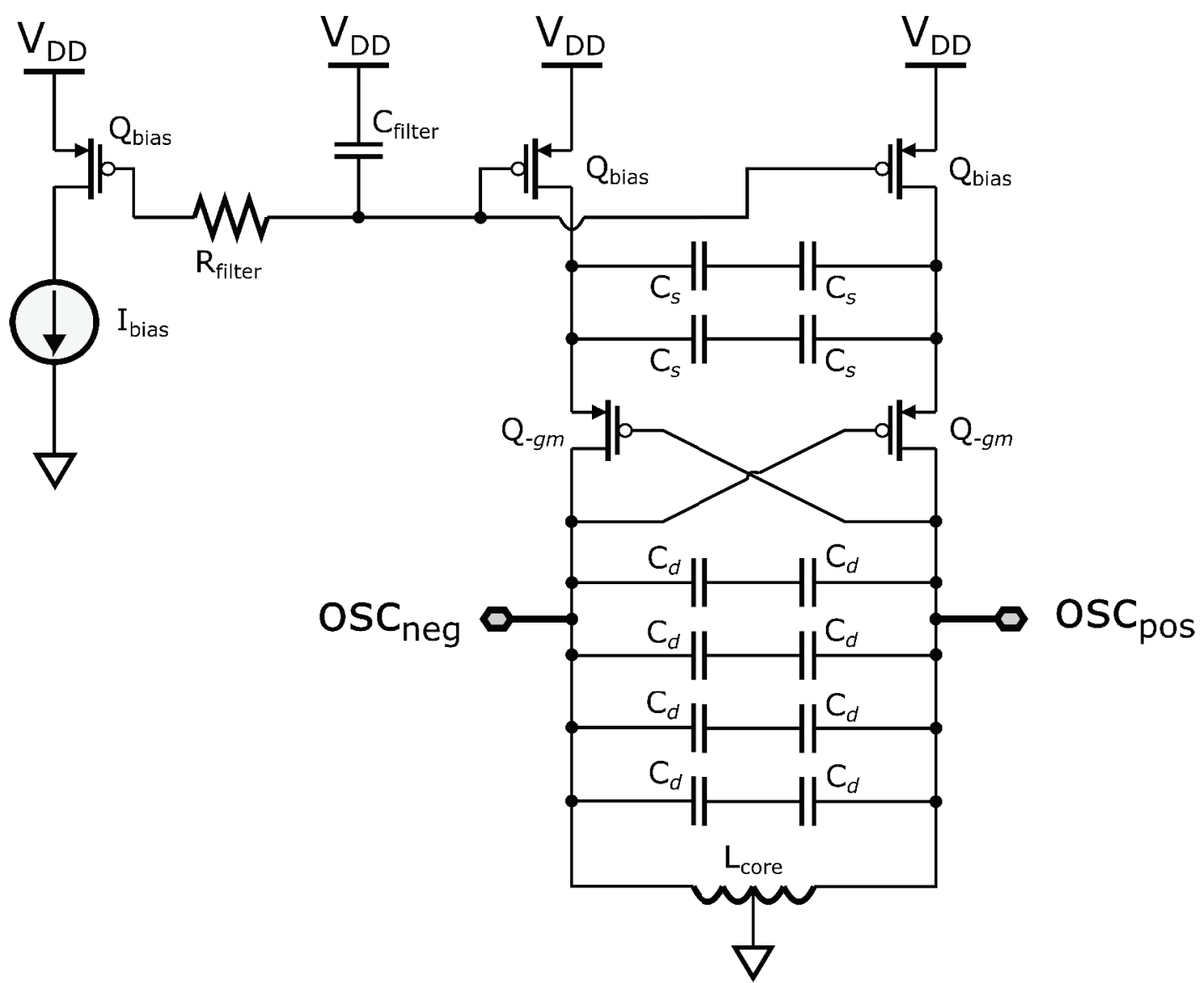

Figure 35: Schematic implementation of the LC DCO core.

An unconventional double current mirror architecture was used (one current mirror on each branch of the oscillator) instead of a single current mirror at the source junction of 
the negative gm transistors (as shown in Figure 23 and Figure 30); this allowed digital banks to be implemented on the source end of the oscillator.

Multiple capacitors were used in parallel on the drain side (instead of one large capacitor) to increase the $\mathrm{Q}$ of the overall tank and reduce the phase noise of the oscillator. Large capacitors with elongated fingers (thus higher resistive fingers) tended to have lower $\mathrm{Q}$ than smaller capacitors with shorter fingers.

Fixed capacitors on the source end of the negative gm transistors were also necessary, for it provided an oscillation pathway for the signal to propagate between the left branch (osc neg) and the right branch (osc pos) of the oscillator core. Multiple capacitors in parallel were also essential on the source end (like the drain side); however unlike the drain side, parallel capacitors on the source end was used not to increase the $\mathrm{Q}$ but to reduce the reactance in the pathway. This was because the largest single capacitor in the kit was not enough.

A low pass RC filter was added at the current mirror junction to reduce noise and spikes/glitches that may arise from outside the system; aiding in current stability and improving phase noise performance.

The core transistors were made as large as possible from available minimum kit size (minimum width and minimum length) to reduce flicker noise (and hence improve the phase noise performance of the oscillator); without being too large so that it can still operate at $7 \mathrm{GHz}$. The transistors were characterized for ft, fmax, and gm using test benches to ensure the conditions of oscillations were met. The width of each core transistor was made large enough to at least draw $15+\mathrm{mA}$ of current; so as to improve the phase noise of the oscillator by increasing the core current if necessary.

The DCO core will be affected (core characteristics such as frequency etc... will change) if a load is applied to the differential output (osc pos and osc neg) of the oscillator. To isolate the core, source followers (common drain or sometimes referred to as unity gain) buffers was added to the outputs of the oscillator. The schematic design of the oscillator buffers in shown in Figure 36. 
The current mirror transistors of the core and the buffer were also made very large to reduce the flicker noise of the transistors and to draw 15+ mA of current (if necessary).

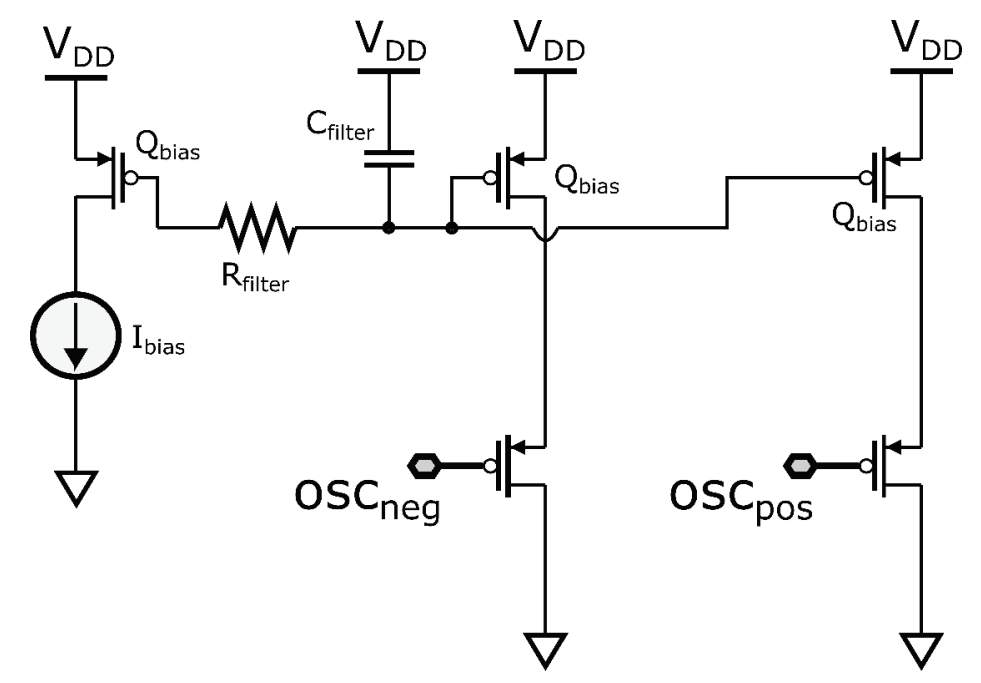

Figure 36: Schematic implementation of DCO core buffers.

The schematic implementation of the DCO Core (Figure 35) and DCO Core Buffers (Figure 36) in the software Cadence Virtuoso Schematic Editor L can be found in Appendix A.

The DCO core by itself produces only one tone (a single frequency), to vary through a wide range of frequencies, digital banks were used.

\subsection{Schematic Implementation of Digital Banks}

Digital banks to switch through the oscillator frequencies, and to increase the frequency range, was implemented using the circuit shown in Figure 37 (Same circuit as the theoretical circuit in Figure 32). 


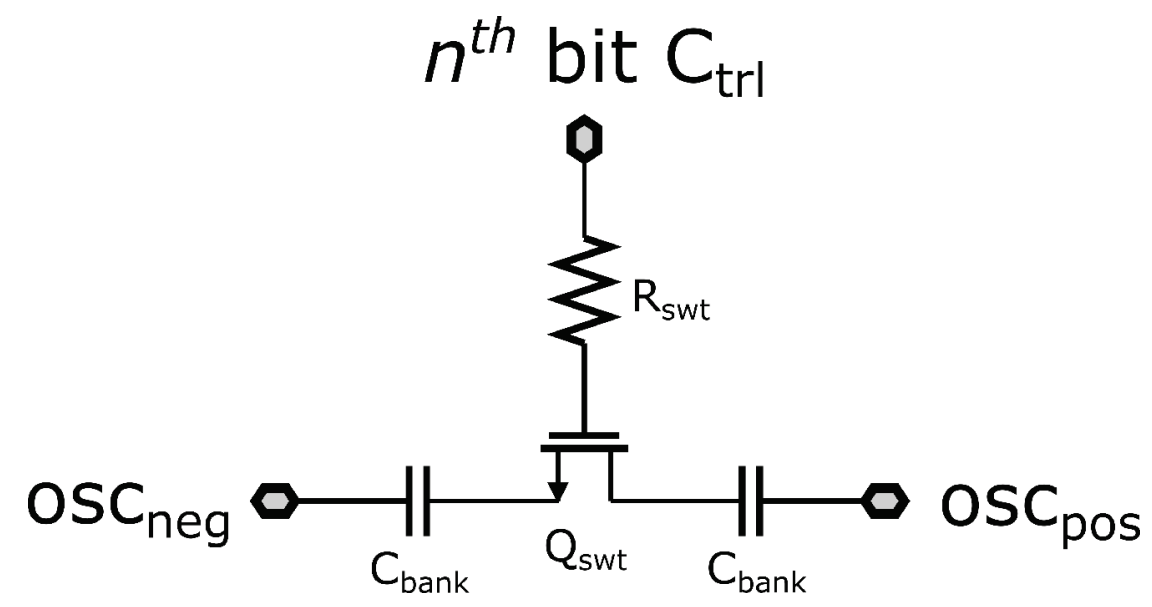

Figure 37: Example schematic of how an oscillator bank was implemented.

The bank circuit in Figure 38 below was initially used; it allowed the node between the two capacitors in the bank to be fully grounded instead of having a floating virtual ground (that can vary), however to reduce components (and hence layout space), and to increase the phase noise performance, the bank architecture was discarded in favour of the bank schematic in Figure 37. See Appendix A for schematic implementation of the banks in Cadence Virtuoso.

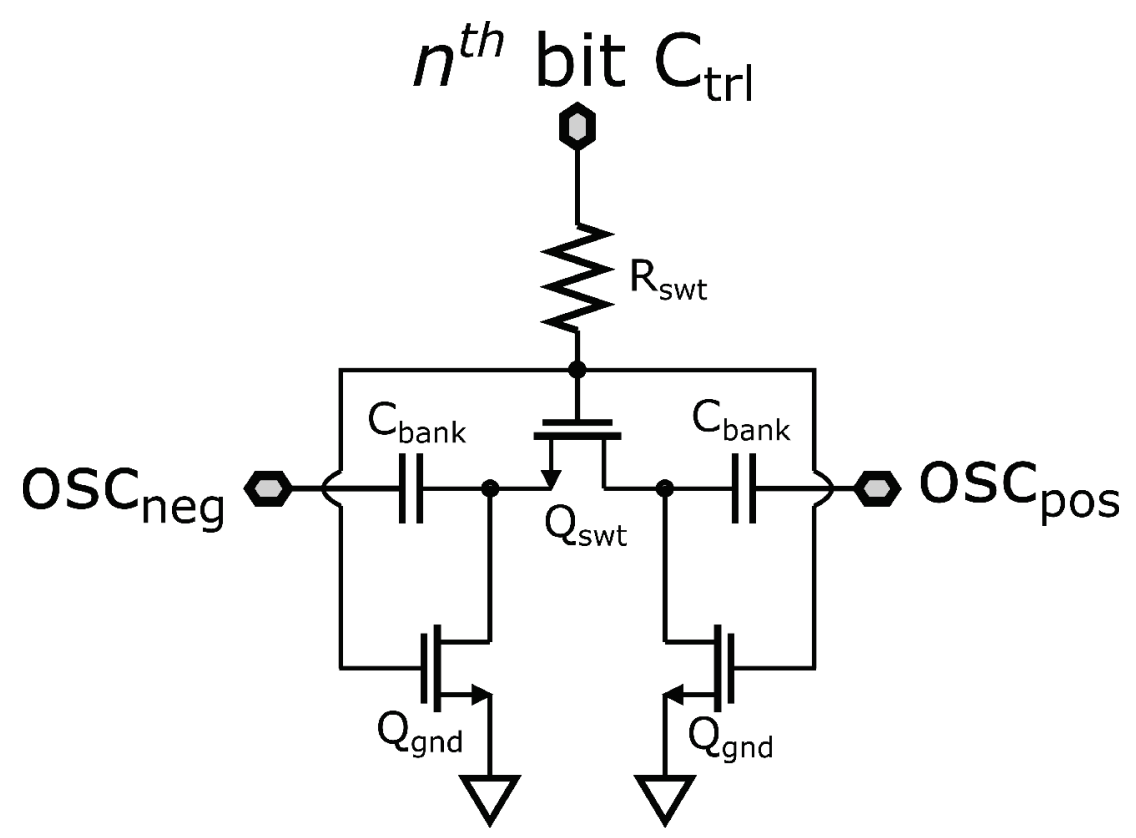

Figure 38: An alternative version of bank configuration that was considered for use in the DCO.

Eleven bank sets were implemented with four sets on the drain side and five sets on the 
source side. Table 2 below describes how many banks were in each set of banks.

TABLE 2: Number of Bank Sets, Banks, and Average Frequency Step Size.

\begin{tabular}{|c|c|c|}
\hline Bank Number & Number of Bank Units & Average Frequency Step Size \\
\hline Bank Set 1 & 16 & $100 \mathrm{MHz}$ \\
\hline Bank Set 2 & 16 & $10 \mathrm{MHz}$ \\
\hline Bank Set 3 & 16 & $2 \mathrm{MHz}$ \\
\hline Bank Set 4 & 16 & $500 \mathrm{kHz}$ \\
\hline Bank Set 5 & 20 & $25 \mathrm{kHz}$ \\
\hline Bank Set 6 & 16 & $10 \mathrm{kHz}$ \\
\hline Bank Set 7 & 16 & $2 \mathrm{kHz}$ \\
\hline Bank Set 8 & 16 & $5 \mathrm{kHz}$ \\
\hline Bank Set 9 & 16 & $1 \mathrm{kHz}$ \\
\hline Bank Set 10 & 16 & $1 \mathrm{kHz}$ \\
\hline Bank Set 11 & 64 & $200 \mathrm{~Hz}$ \\
\hline
\end{tabular}

All banks within each bank sets were identical. The capacitance of the banks with progressive bank sets were smaller and smaller so that finer frequency steps can be achieved. The capacitance of the banks when it is off $\left(\mathrm{C}_{\text {off }}\right)$ is smaller then when it is on $\left(\mathrm{C}_{\mathrm{on}}\right)$. MOM capacitors were used for the banks. Since the capacitance of MOM capacitors are independent of voltage (unlike varactors), the value of $\mathrm{C}_{\text {off }}$ or $\mathrm{C}_{\text {on }}$ should stay relatively the same even though the swings of the oscillator will be large during the duration of oscillation. A bank with a transistor switch can be modelled by the schematic shown in Figure 39.

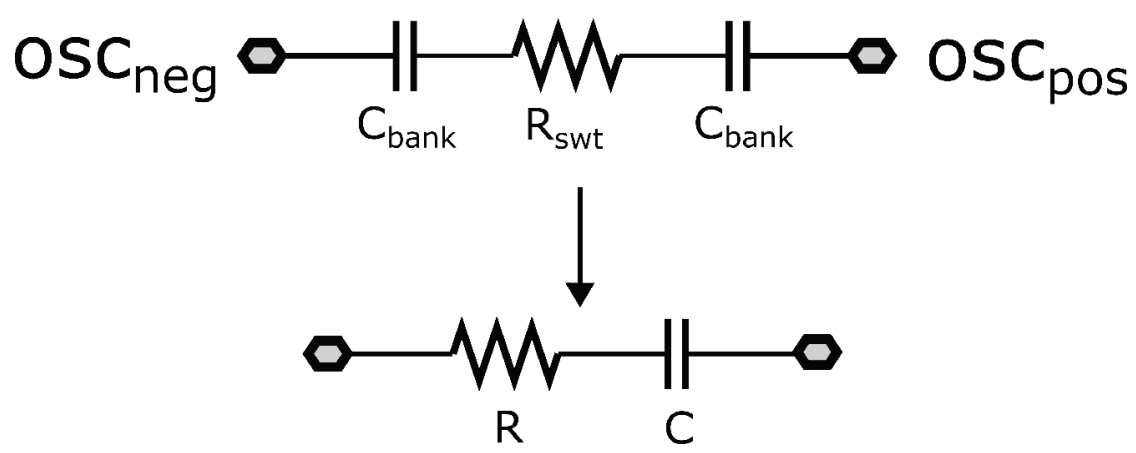

Figure 39: Modelling bank capacitors and switch transistor using series RC.

When the transistor switch is off, $\mathrm{R}_{\mathrm{swt}}$ is very large (and for simple analysis) can be treated as an open circuit. When the transistor is on, $R_{\text {swt }}$ is the channel resistance and will vary depending on the large signal swings between osc $\mathrm{pos}$ and $\mathrm{osc}_{\text {neg. }}$.

The $\mathrm{Q}$ of a series $\mathrm{RC}$ circuit is given by: 


$$
Q=\frac{1}{\omega_{0} R C}
$$

The $\mathrm{Q}$ of the bank will vary depending on frequency of the oscillator and the transistor region of operation. Increasing the $\mathrm{Q}$ of the bank will increase the overall phase performance of the oscillator. It can be observed from Eq. 48 that the Q of the bank can be increased by reducing the series $\mathrm{R}$. This can be achieved by using the minimum transistor length and increasing the width of the transistor to reduce the channel resistance (i.e. increase W/L ratio). It should be noted however that once the transistor is made large enough, the total $\mathrm{Q}$ of the banks is limited by the capacitors and no longer limited by the channel resistance (since the channel resistance approaches a short circuit). It was experimentally observed that the optimal size of the transistor width was slightly larger than the width of the capacitors attached to the switch transistor, any larger and the returns for the total bank Q were diminishing (see Figure 49 and Figure 50 in Chapter 6). The transistor widths were different for banks in one bank set to another since the capacitors sizes were different; however all capacitors and transistors were the same for all banks within a bank set.

At all times the switch of the bank (gate of switch transistor in the bank) was either tied up to high (VDD) for a digital high bit 1 or low (GND) for a digital low bit 0 to ensure the switch was not floating. This also ensured the charge within the gate capacitance of the switch transistor was fully discharged when opened and not leaking when closed.

Even with all banks on, the oscillator frequency was too high for automated test equipment. To reduce the frequency for proper measurements, current mode logic (CML) dividers were used to divide the oscillator frequency.

\subsection{Schematic Implementation of CML Dividers}

CML dividers were used in conjunction with the oscillator since static dividers using flip flops (or even dynamic dividers) were not fast enough to divide the high frequency output of the oscillator. The schematic implementation of the CML divider used is shown in Figure 51. See Appendix A for the schematic implementation of the CML divider in Cadence Virtuoso. 


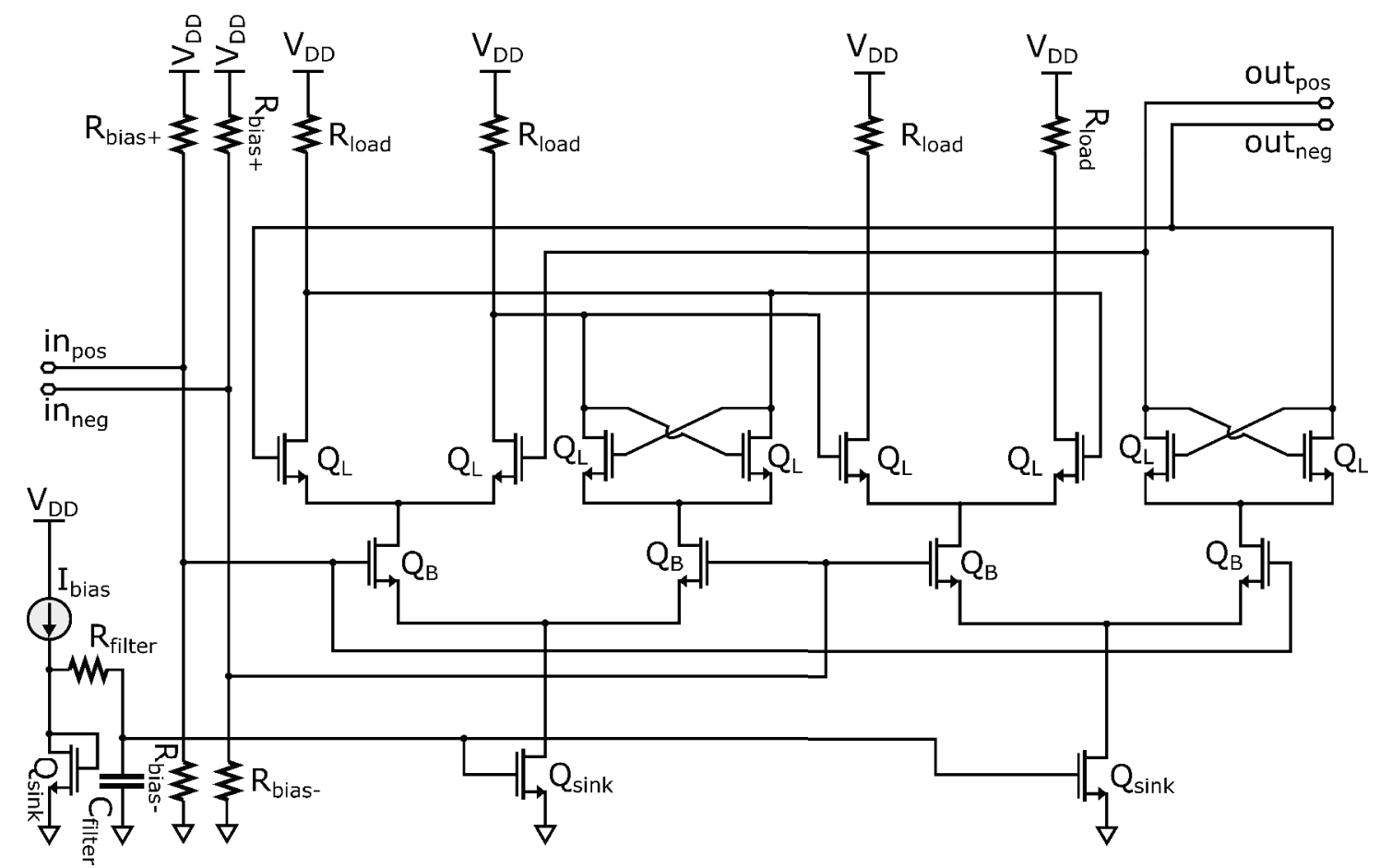

Figure 40: CML divider schematic used to divide the DCO frequency in half. The schematic was ported from a generic CML and re-designed by the author.

Four CML dividers were used in series to divide the oscillation frequency by $2,4,8$, and 16 , and in between each CML, source follower buffers were used the isolate the CMLs. Large source follower buffers were also used leading up to the external pads from the CMLs.

\subsection{Voltage Regulator}

A modified in-house Microsemi (Microchip) Corporation on-chip voltage regulator was used to power the DCO, however due to confidentiality, the schematic of the regulator will not be disclosed in this thesis.

\subsection{Digital Circuit to Control DCO}

The DCO requires too many digital bits to control the digital banks. In reality the number of pads connecting the DCO to the external ecosystem is limited. Not enough pads were available for all the digital bits (required by the DCO) unless a ball grid array packaging was used; and even then it may have not been enough. To resolve the issue, a two method contraction system was used to reduce the number of bond pads required to one. First, a thermometer to binary decoder was used to reduce 228 control bits going into the oscillator to 57 bits, and then the 57-bits were reduced to 1-bit by using a serial to parallel interface 
(termed in this thesis as S2PI). See Figure 41 for a system level diagram of how the reduction of bond pads was achieved.

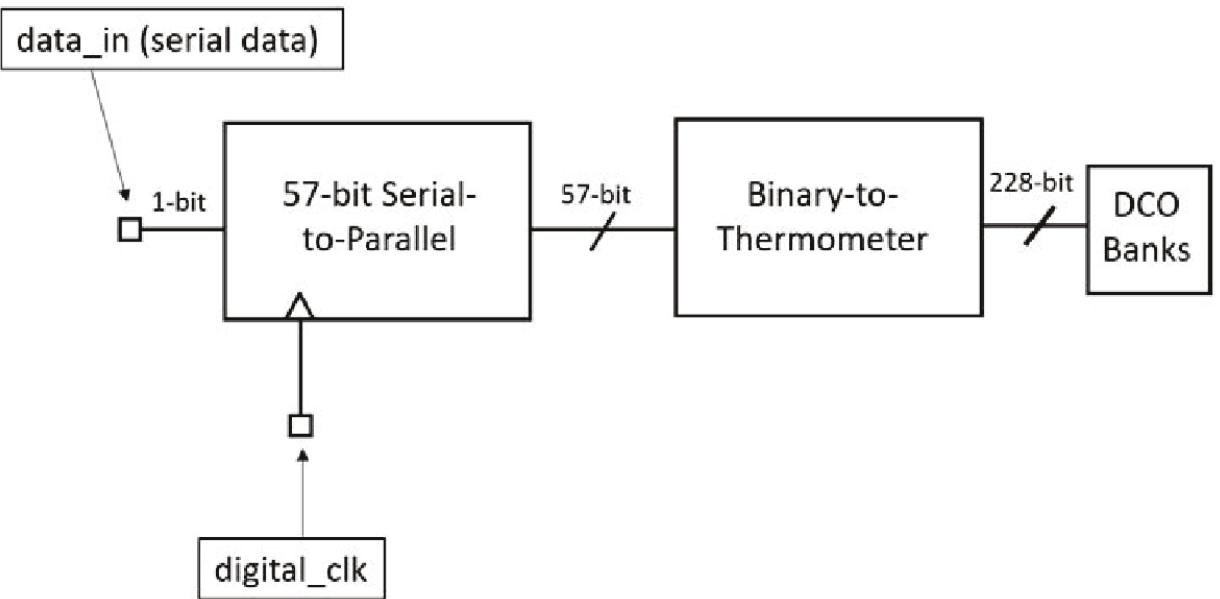

Figure 41: Two stage method to reduce DCO digital control bits from 228 bits to 1 bit.

\subsubsection{Binary to thermometer decoders}

Different bit binary to thermometer converters were used, however they all used the same logic principle. An example of a schematic of a 2 bit to 3 bit binary to thermometer converter is shown in Figure 42. See Appendix A for other binary to thermometer schematics.

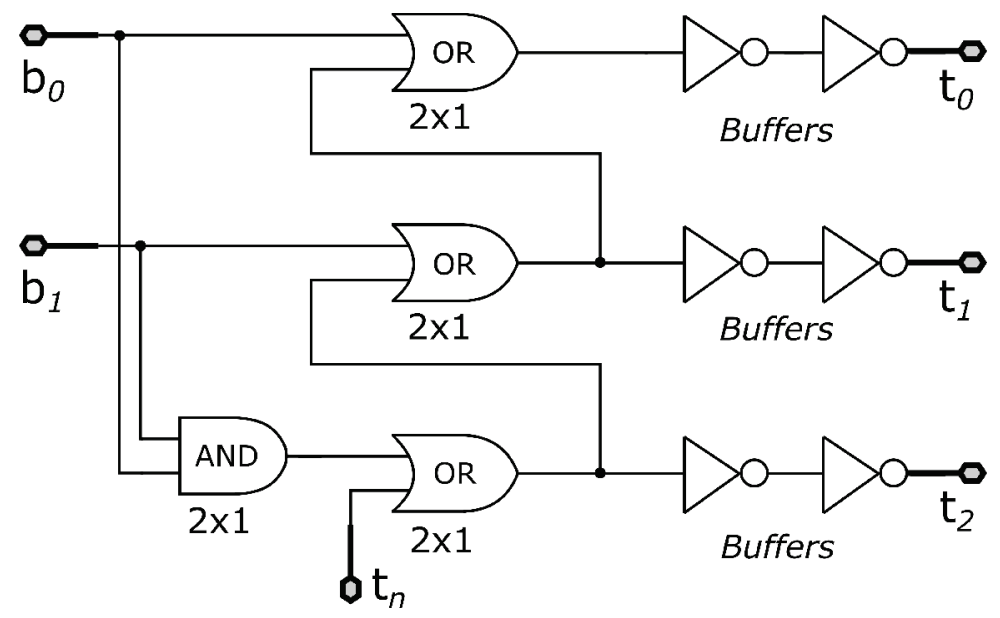

Figure 42: A 2-bit to 3 bit binary to thermometer decoder. Binary inputs are on the left, and thermometer outputs are on the right. Schematic was designed from scratch by the author. 


\subsubsection{Serial to parallel interface $(\mathrm{S} 2 \mathrm{PI})$}

The custom made Serial to Parallel interface (S2PI) was composed of a series of shift registers like the one shown in Figure 43. Each shift register was made up of a regular static D-flip-flop shown in Figure 44. See Appendix A for the schematic implementation in Cadence Virtuoso.

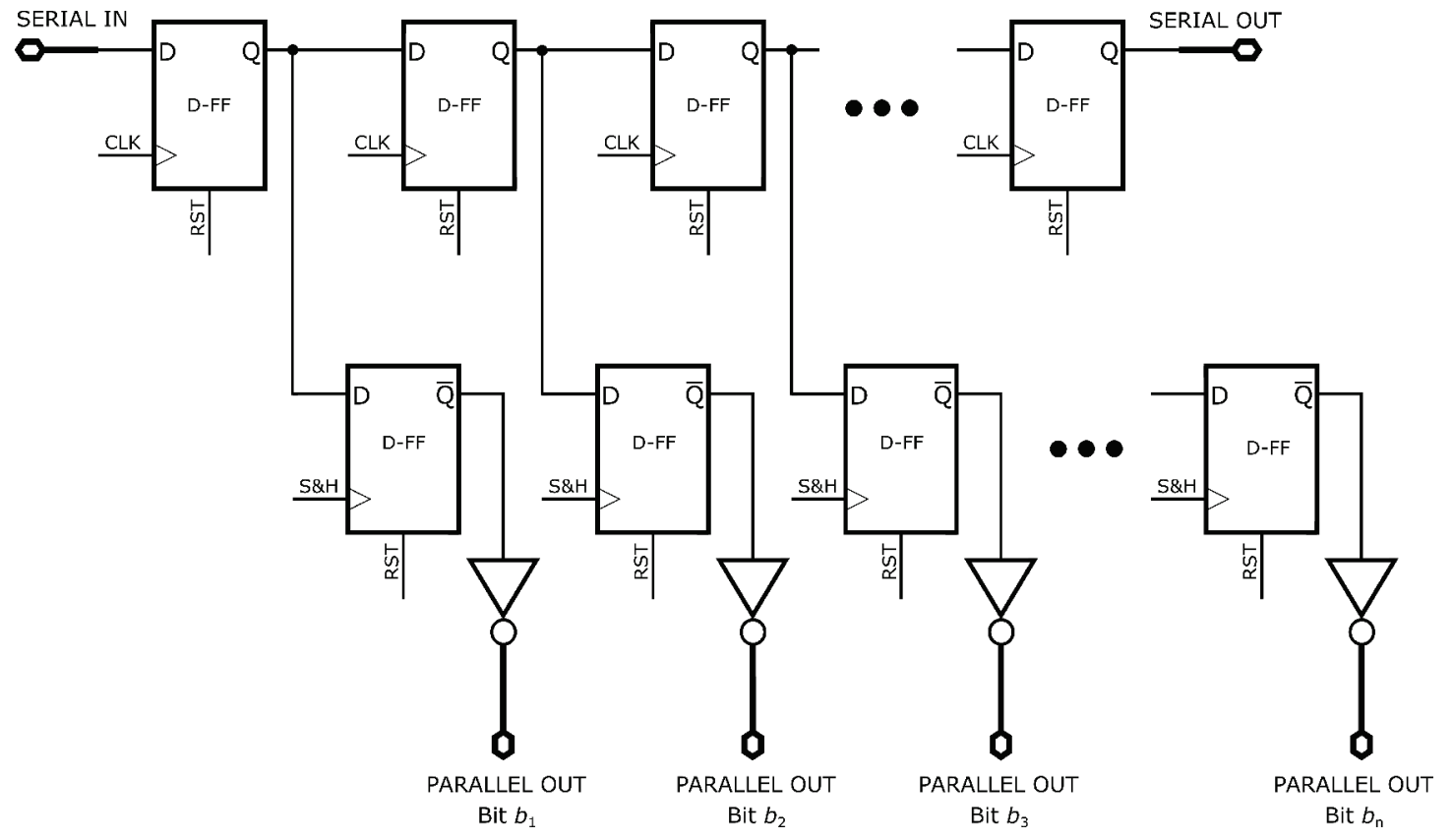

Figure 43: Schematic implementation of serial to parallel interface. Circuit was designed from scratch by the author based on a generic serial to parallel interface schematic.

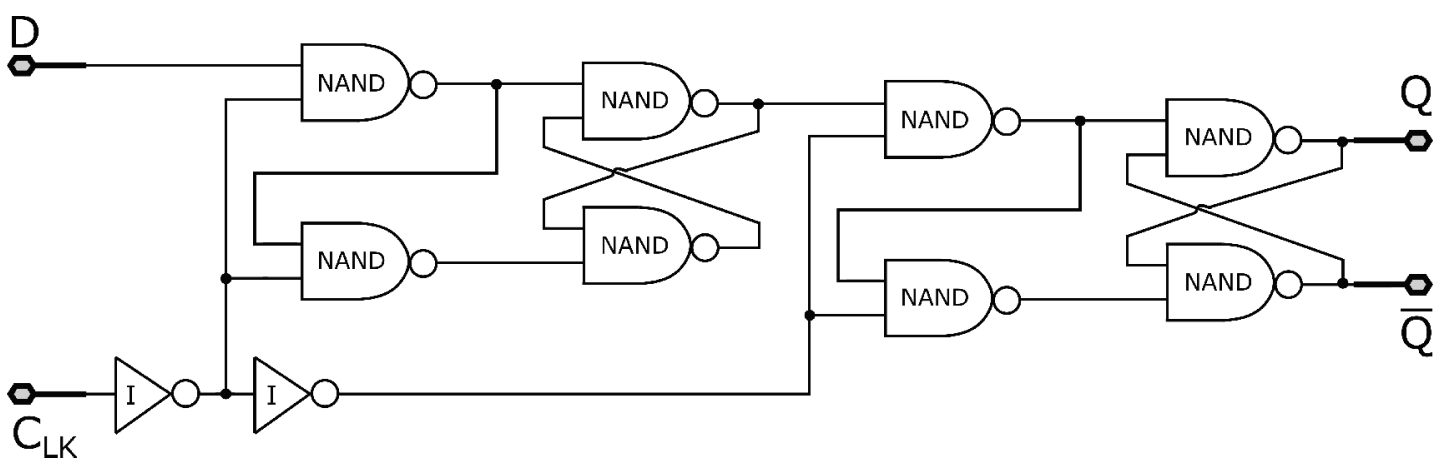

Figure 44: Schematic of a flip-flop used as a shift register. Schematic was ported from a generic D flip-flop. 


\subsubsection{Muxes}

An in-house Microsemi (Microchip) Corporation Serial to Peripheral interface (SPI) that would interact with a digital loop filter in an ADPLL loop was also used to control the DCO. To distinguish the control lines from the in-house SPI and custom made S2PI, muxes were used. Pass gate muxes were chosen for conserving layout footprint and increasing speed performance. An example of a typical mux used is shown in Figure 45 and it's schematic implementation in Virtuoso can be found in Appendix A.

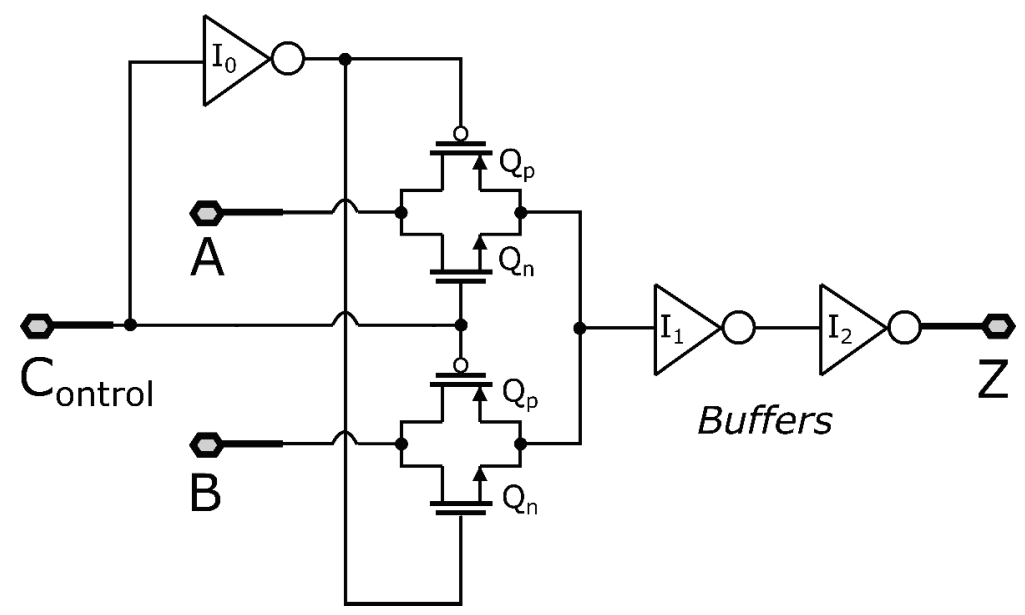

Figure 45: A 2 input mux using pass transistors.

\subsubsection{Ring Oscillator}

A ring oscillator that can be enabled and disabled inside the chip was also designed. Dividers were used to divide the frequency of the ring oscillator to $1 \mathrm{MHz}$ (see Figure 46). Inverter chain buffers were used to drive any digital logic inside and outside the chip. 


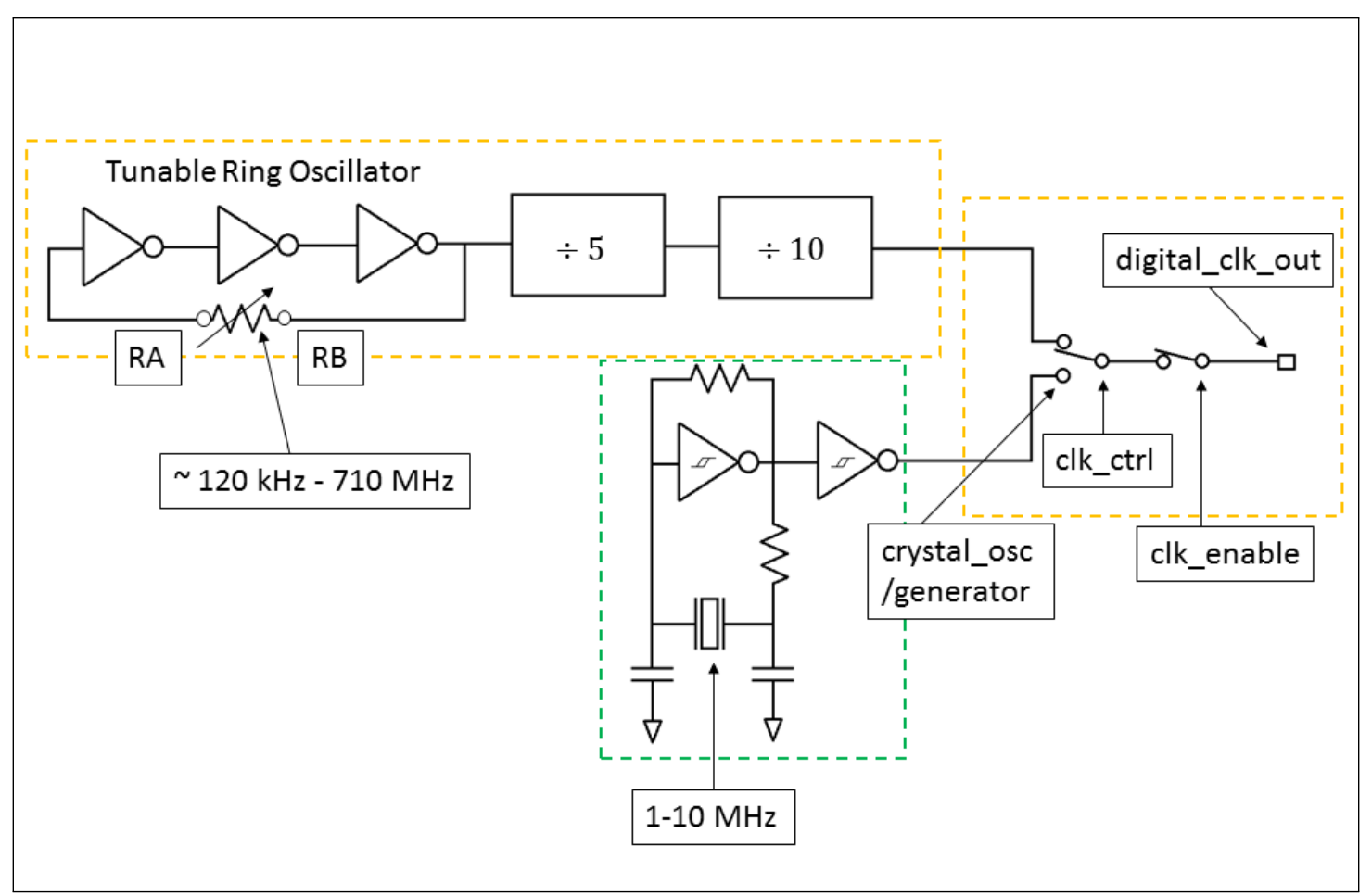

Figure 46: Clock mechanism to time the internal digital circuit

\subsection{Conclusion}

This chapter looked at the schematic design of the DCO (proposed in this thesis) and its peripheral circuitry. The chapter looked at the schematic design of the DCO core, DCO core buffers, and the schematic implementation of the digital banks. The chapter also looked at the schematic design of current mode logic dividers, thermometer to binary decoders, serial to parallel converters, and dynamic muxes. 


\section{CHAPTER 6: LAYOUT DESIGN}

This Chapter will present and highlight the layout designs of the DCO circuit; along with the layouts of all other circuits that were crucial for the functioning of the DCO. The layout designs were all drawn in Cadence Virtuoso Layout (XL) using the $40 \mathrm{~nm}$ TSMC kit. The technology kit had 8 metal layers for routing, 2 poly layers, a top aluminium layer (AP) for bond pads, built-in inductors, MOM capacitors and varactor moscaps.

***Note: All layouts in this Chapter were designed by the author with the exception of an in-house Microsemi (Microchip) voltage regulator that was modified by the author, a DLF which was designed by Krste Mitric, and a TDC which was designed by Tony Wang.

\subsection{DCO Tank, Digital Banks, and Core Buffer Layout}

The LC core layout of the DCO, along with its banks on the drain and source side, and the DCO source follower buffers are shown in Figure 47.

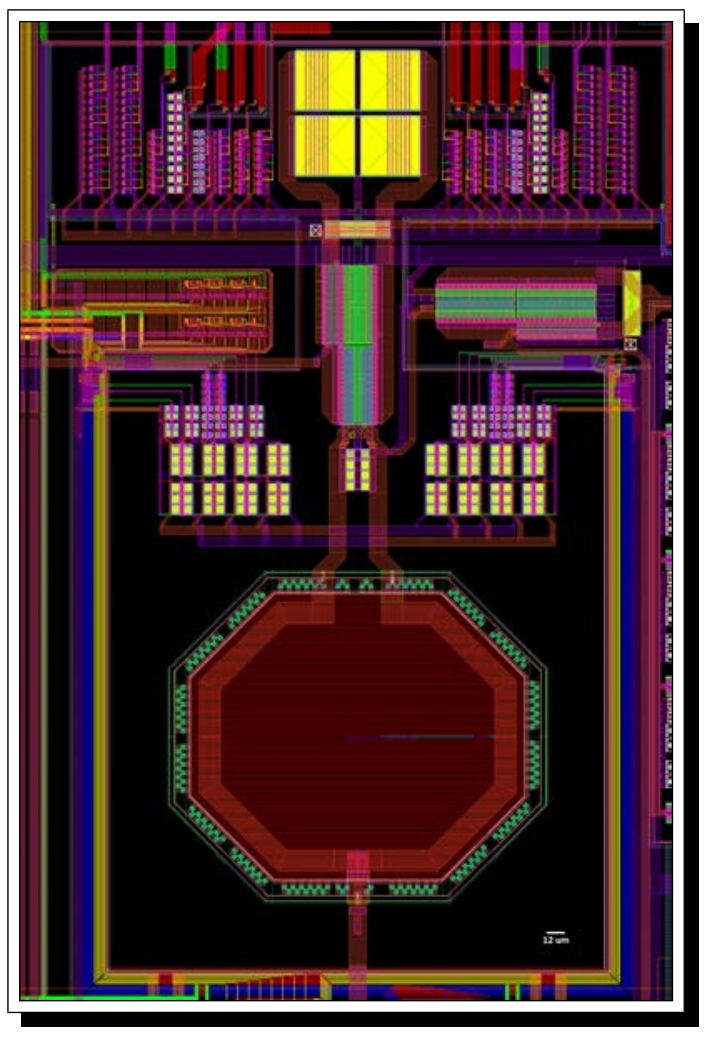

Figure 47: DCO core layout, along with the DCO buffers, drain banks, and source banks. The size of the DCO core was $400 \mu \mathrm{m} \times 600 \mu \mathrm{m}$. ***Note: Metal fills are not shown for clarity. 
Improving the $\mathrm{Q}$ of the inductor was crucial since it was one of the main factors inhibiting the oscillators performance. As can be observed in Figure 47, the inductor takes up almost half the real estate of the DCO core. On chip inductors are generally lossy and reduces the phase noise performance of the oscillator. The thickest metal layer available in the kit was used for the inductor to reduce it's internal resistance.

A custom made inductor (shown in Figure 48) was initially used for the DCO. The custom inductor was created using the aid of EMX (electromagnetic simulator software) [45]. However, an almost identical kit inductor was found and used in its place. The advantage of using the kit inductor was that it had guard rings already built in, improving the noise immunity of the inductor from substrate leak.

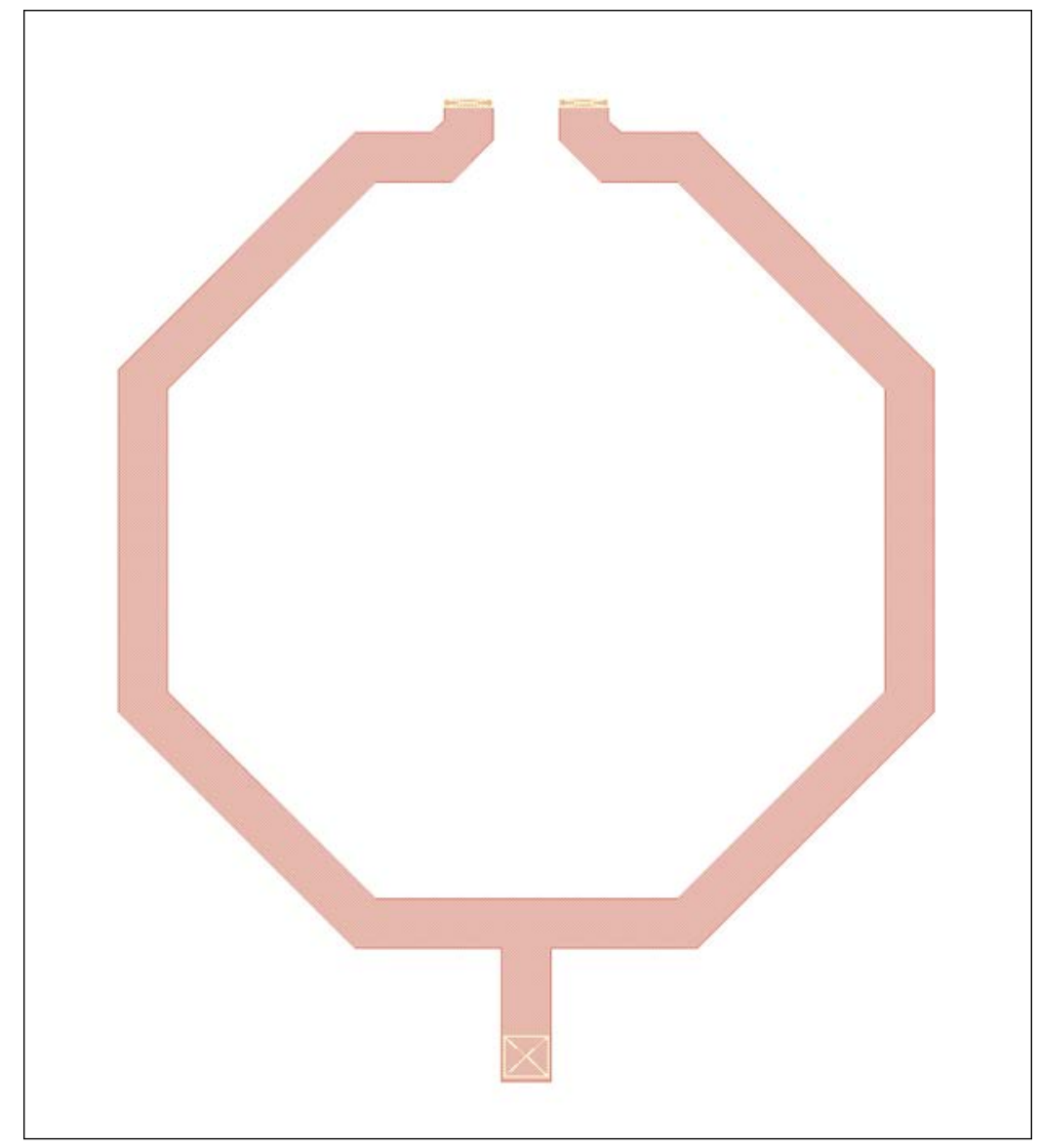

Figure 48: Design of an inductor intended to be used for the DCO. The size of the inductor was $240 \mu m$ by $240 \mu m$. 


\subsubsection{Layout of Digital Banks}

A couple examples of the layout design of digital banks is shown in Figure 49 and Figure 50. Figure 49 shows the layout of a bank from Bank set 3 and Figure 50 shows the layout of a Bank set 2. The two different bank layouts from Figure 49 and Figure 50 will have different quality factors $\mathrm{Q}$ depending on the capacitor values, the $\mathrm{W} / \mathrm{L}$ ratio of the switch transistor, and the RC parasitic of the metal traces connecting the components of the bank together. Note that all the bank layouts are the same for all banks within a bank set but the bank layouts are different from bank set to bank set.

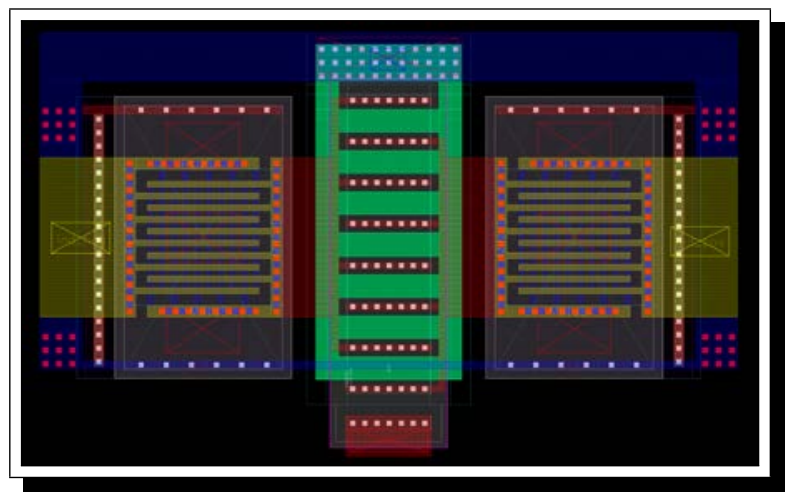

Figure 49: An example layout of a digital bank. The size of the bank was $8.3 \mu \mathrm{m} \times 4.1 \mu \mathrm{m}$.

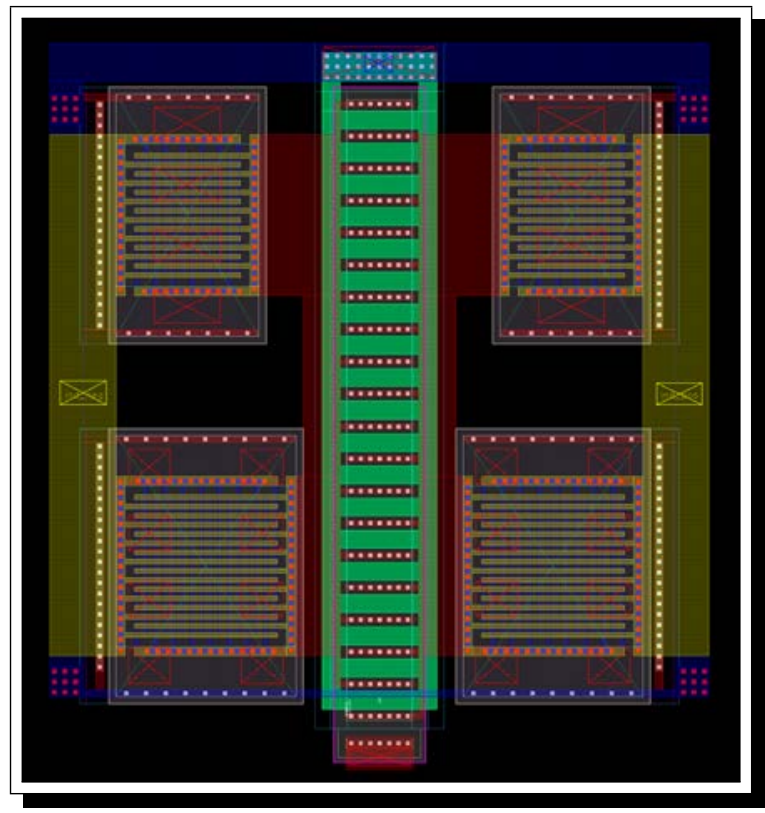

Figure 50: An example layout of a digital bank using multiple capacitors in parallel. Size of the bank was $10 \mu m \times 11 \mu m$. 
Again, multiple capacitors were used in parallel in some of the banks to increase the Qs of the overall bank. Multiple capacitors in parallel/series were also used to achieve precise capacitance requirement (that could not have been achieved using single capacitors governed by parameters that were constrained by the kit). The switch transistor width for the banks in Figure 49 and 50 were slightly larger than the size of the capacitors attached to it (as previously discussed in Chapter 5.2 for attaining optimal Q of the banks).

\subsection{Divider Layout}

The layout of a single CML Divider is shown in Figure 51. The metal layers had to be large enough to draw enough current so as to not reduce the phase noise performance of the oscillator; but at the same time had to be thin enough so as to not add additional parasitic that can slow the performance of the CML. Thin metal traces also caused problems since they acted as DC blocks at higher frequencies (due to higher inductance). This was mostly an issue for the first couple of CMLs as the progressive CMLs in the chain had to deal with lower and lower frequencies.

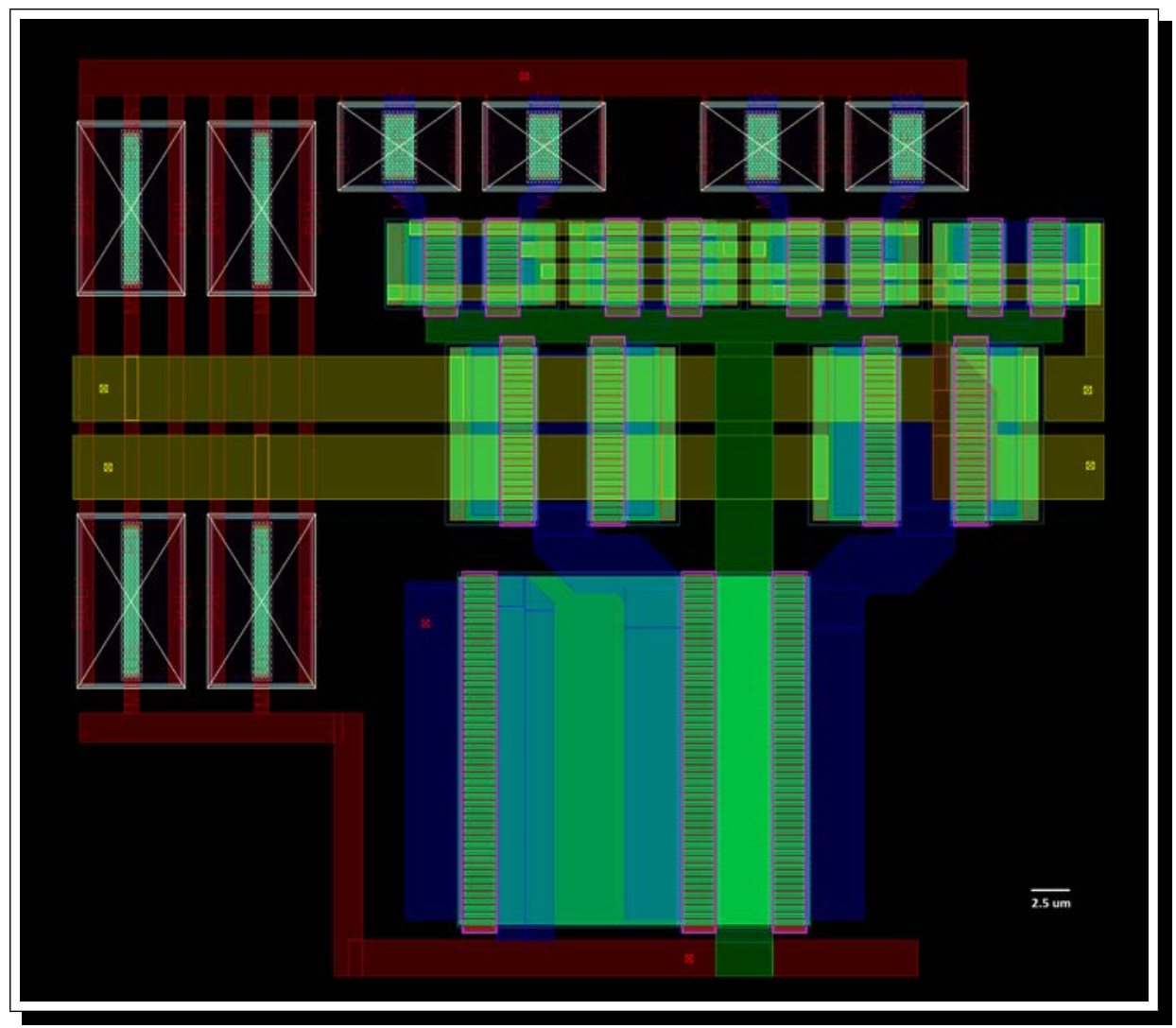

Figure 51: A layout of a CML used to divide the DCO frequency by half. Size of the CML was $72 \mu m \times 64 \mu m$. 
The layout of the full CML chain (four CMLs in series dividing the output frequency of the oscillator by $2,4,8$, and 16) along with unity buffers in between them for signal padding and isolation is shown in Figure 52.

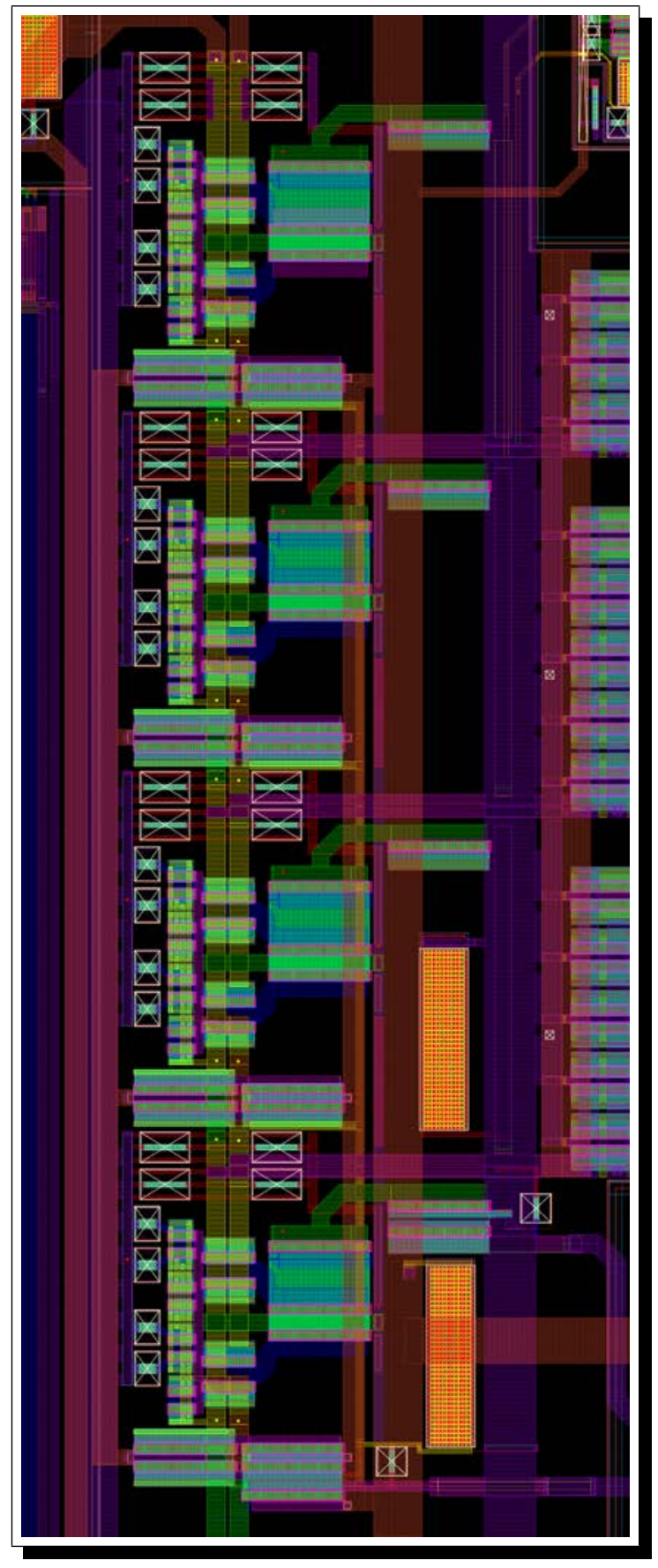

Figure 52: Layout of four CML dividers in series. CMLs were used to divide the oscillator frequency by 2,4,8 and 16. Buffers are placed in between the CMLs to drive the signals from the CMLs. DCO signal is propagating from top to bottom in the image above. 


\subsection{Layout of Buffers Connecting to the Bonding Pads}

Large common drain buffers were needed to drive the signal from the CMLs to the pads and to the external world. The driving load requirement was $50 \mathrm{Ohms}$ (commonly used by testing equipment). The power levels needed to be high enough (to be above the noise floor) so the oscillating signal can be observed on spectrum analysers and counters. The layout of the a buffer connecting to the bonding pad is shown in Figure 53.

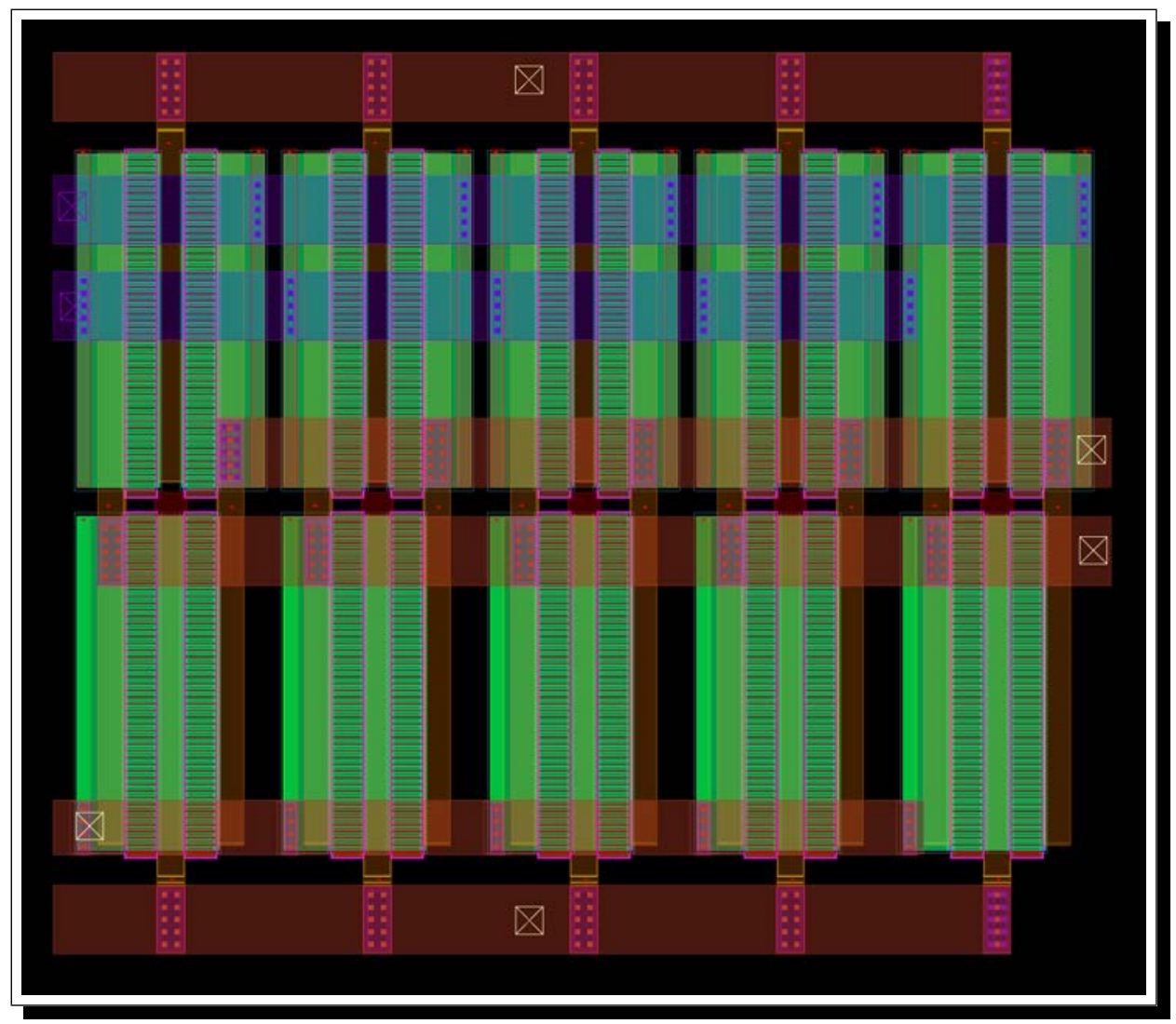

Figure 53: Layout of large common drain buffer used for connecting to the bonding pads. The size of the buffer was $77 \mu m \times 66 \mu m$.

\subsection{Layout of Binary to Thermometer Decoder}

As stated previously in the prior chapter, thermometer to binary decoders were needed to reduce the bond pad requirement for digital control inputs. An example of a 5-16 bit binary to thermometer decoder is shown in Figure 54. 


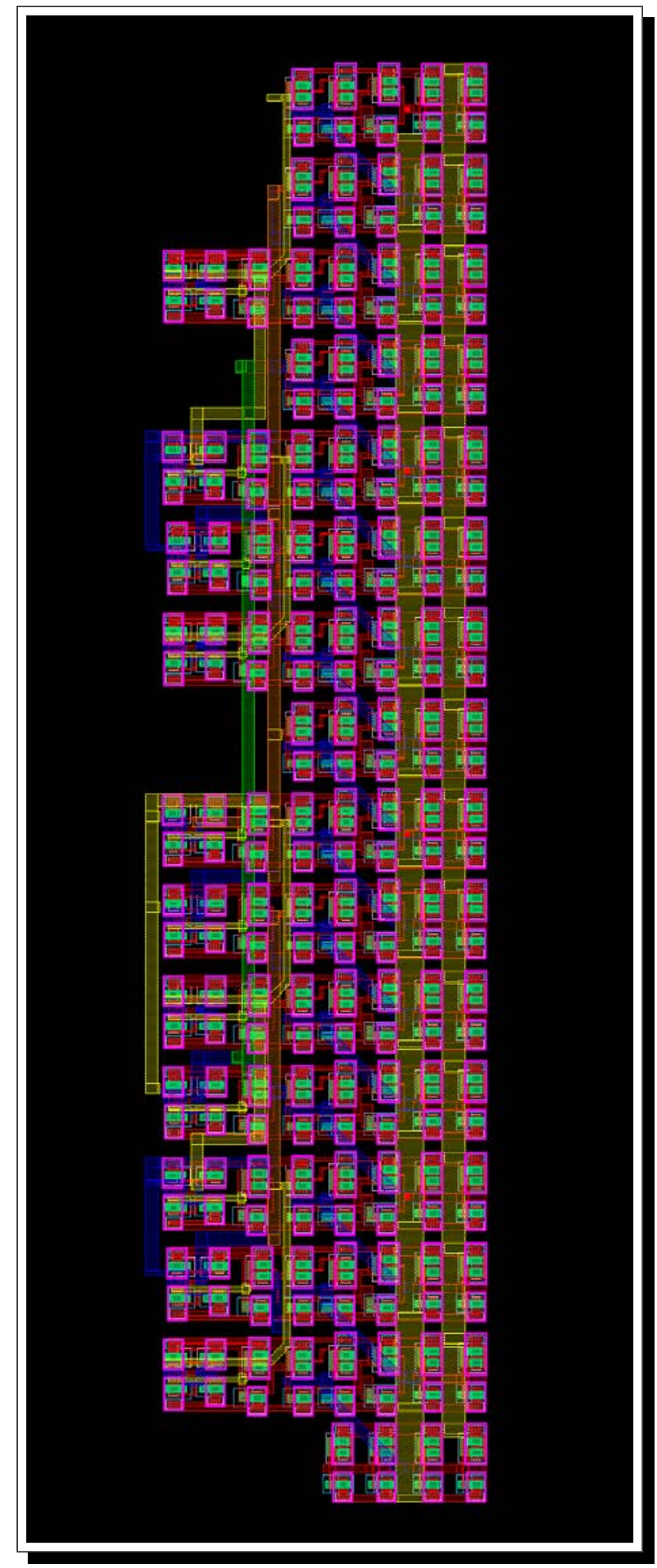

Figure 54: A layout of a binary to thermometer decoder. This specific layout is the layout of a $5-16$ bit converter. The size of the layout is $15 \mu \mathrm{m} \times 62 \mu \mathrm{m}$. 


\subsection{Layout of 57 Bit Serial to Parallel Converter}

The layout of the 57 bit serial to parallel converter which was used to reduce the number of digital binary control inputs of the DCO to one is shown in Figure 55.

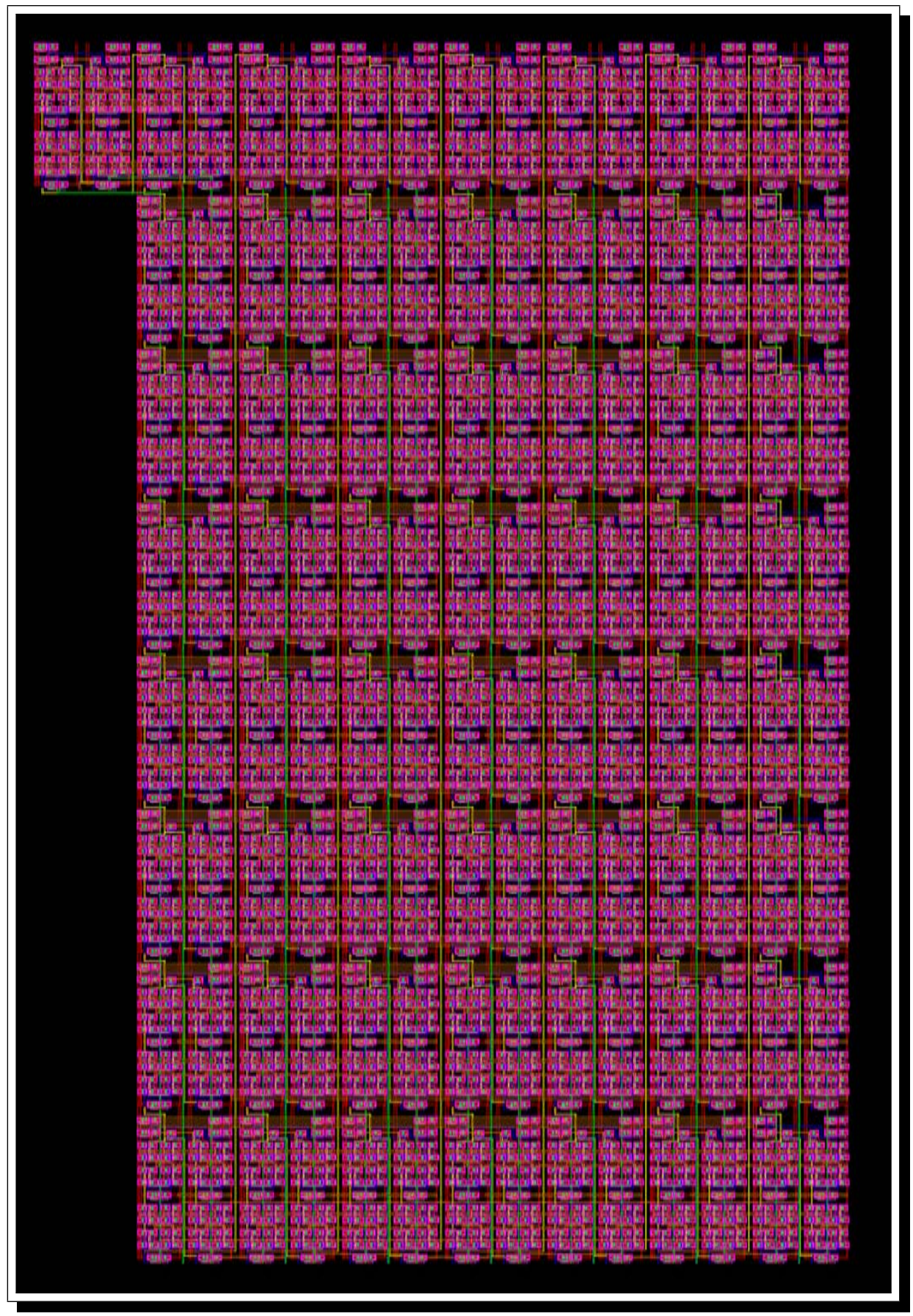

Figure 55: Layout of 57 bit serial to parallel converter. The size of the layout was $120 \mu \mathrm{m}$ $\mathrm{x} 180 \mu \mathrm{m}$. 


\subsubsection{Layout of Shift-Register}

The layout of an individual shift-register used in the serial to parallel converter is shown in Figure 56 .

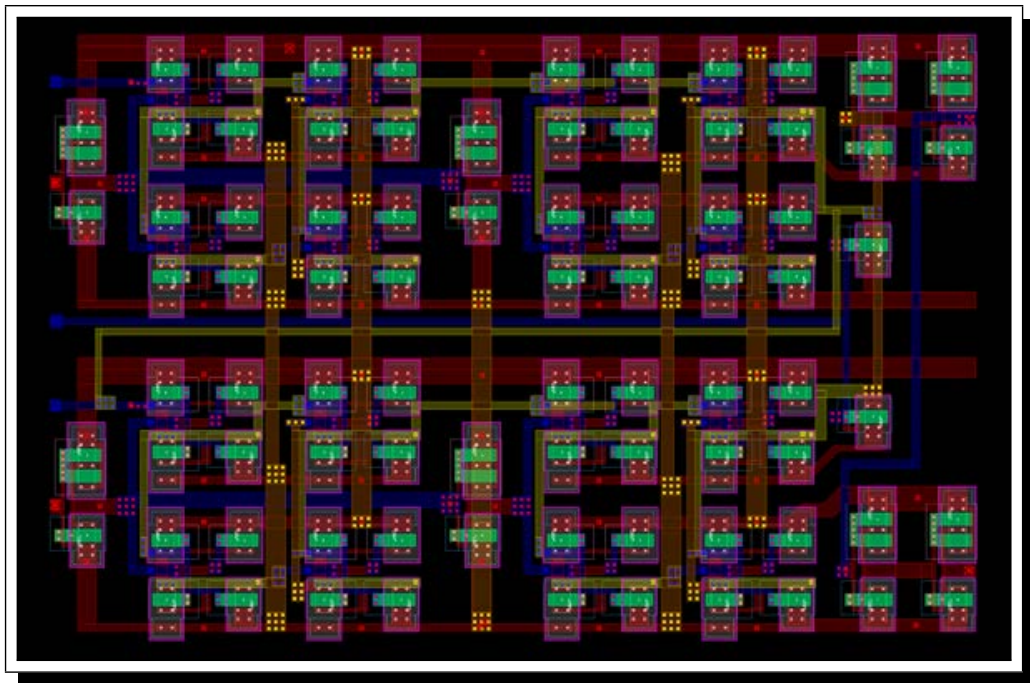

Figure 56: Layout of a single flip flop used in the serial to parallel converter. The size of the layout was $22 \mu \mathrm{m} \times 14 \mu \mathrm{m}$.

\subsection{Layout of Digital Clock and Dividers}

Layout of the built in internal clock mechanism (ring oscillator) along with divide by 5 and divide by 10 logic to drive the digital circuit is shown in Figure 57.

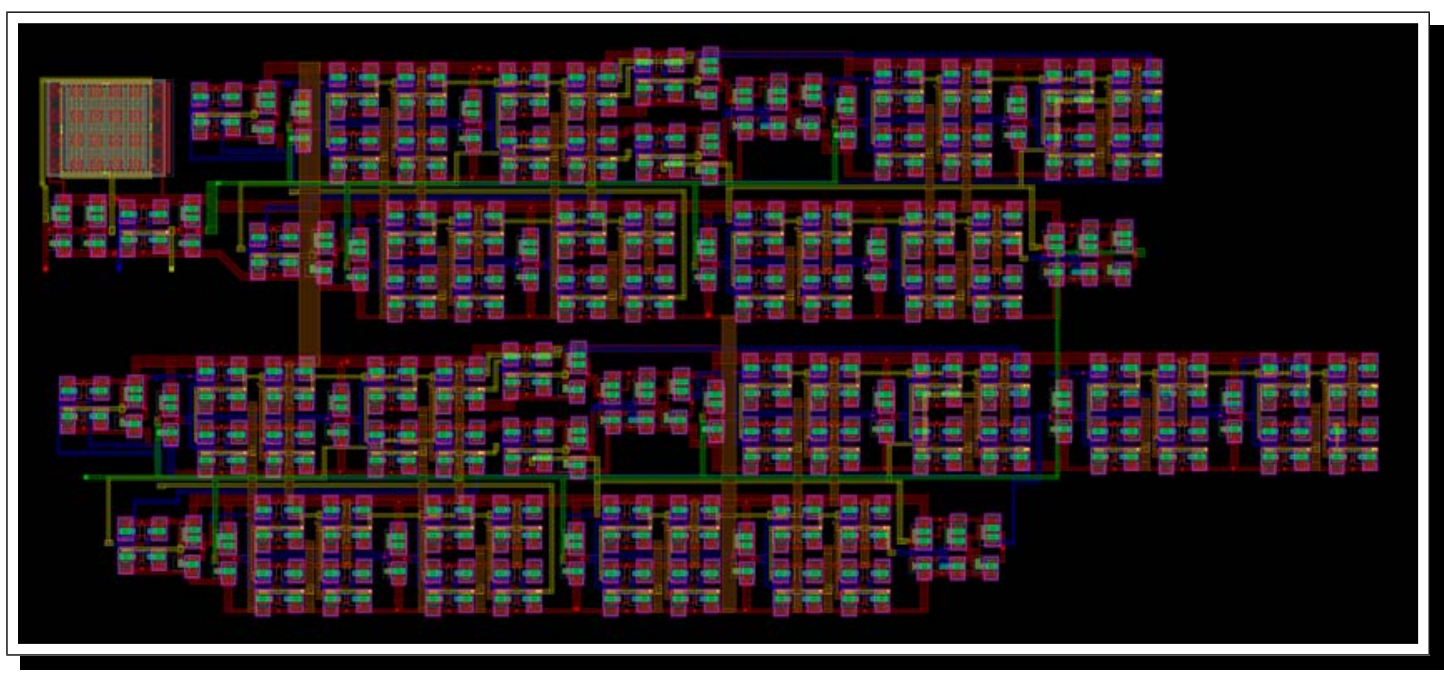

Figure 57: Layout of ring oscillator clock and frequency dividers for clocking the digital signals of the DCO. Size of the layout was $73 \mu \mathrm{m} \times 31 \mu \mathrm{m}$. 


\subsection{Layout of the DCO With Digital Circuitry}

The layout of the DCO with CML drivers, buffers, regulator, digital clock, dividers, decoders and all other digital circuitry is shown in Figure 58 (with the various compartments labelled). Figure 59 shows a higher resolution image of the DCO and its compartments without any label for clarity. All traces connecting the DCO were manually routed (including the traces that were placed in between the decoders) to insure symmetry and proper thickness.

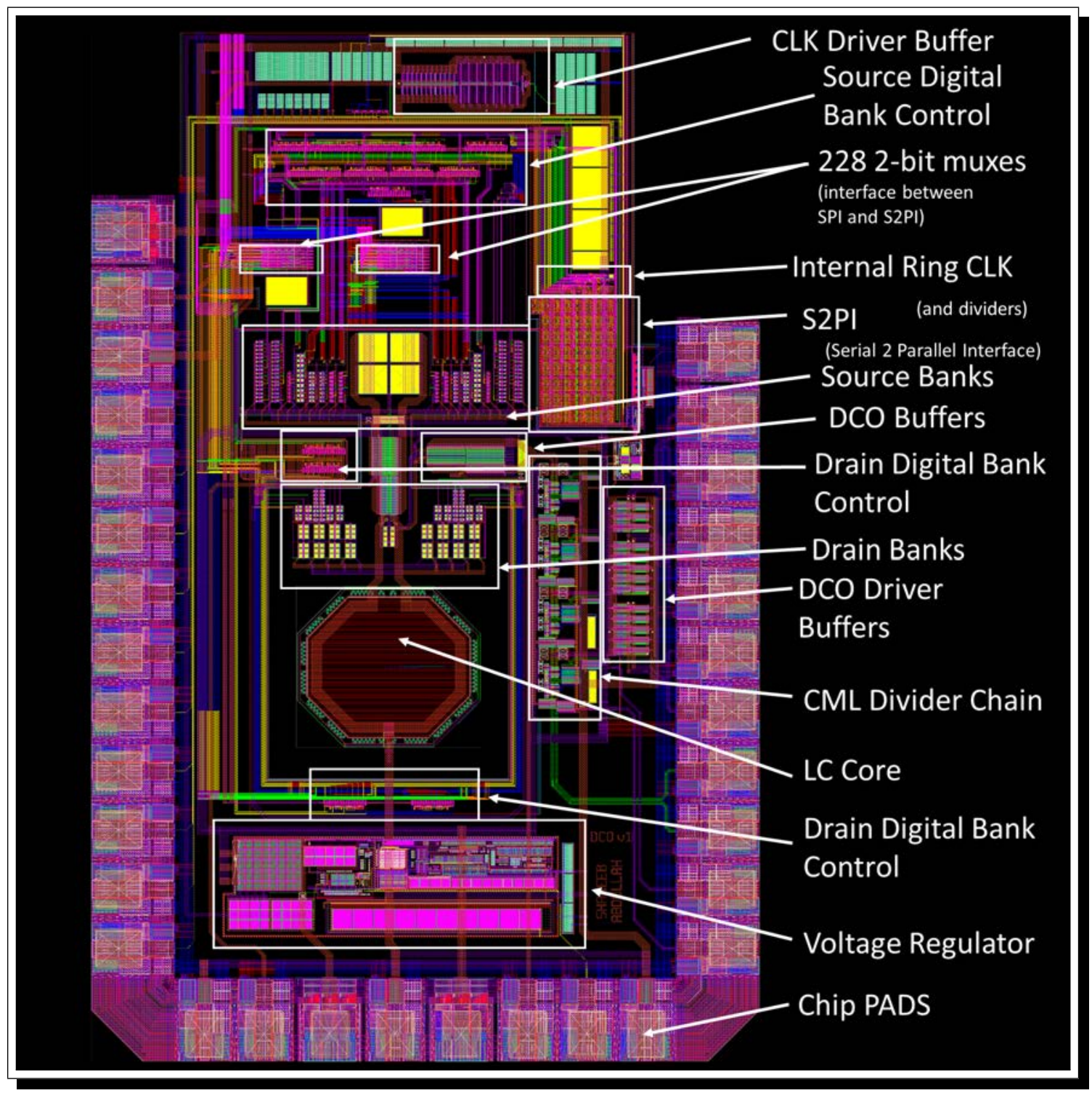

Figure 58: Layout of the DCO with digital compartments labelled. Size of the layout was $958 \mu m \times 1477 \mu m$. 


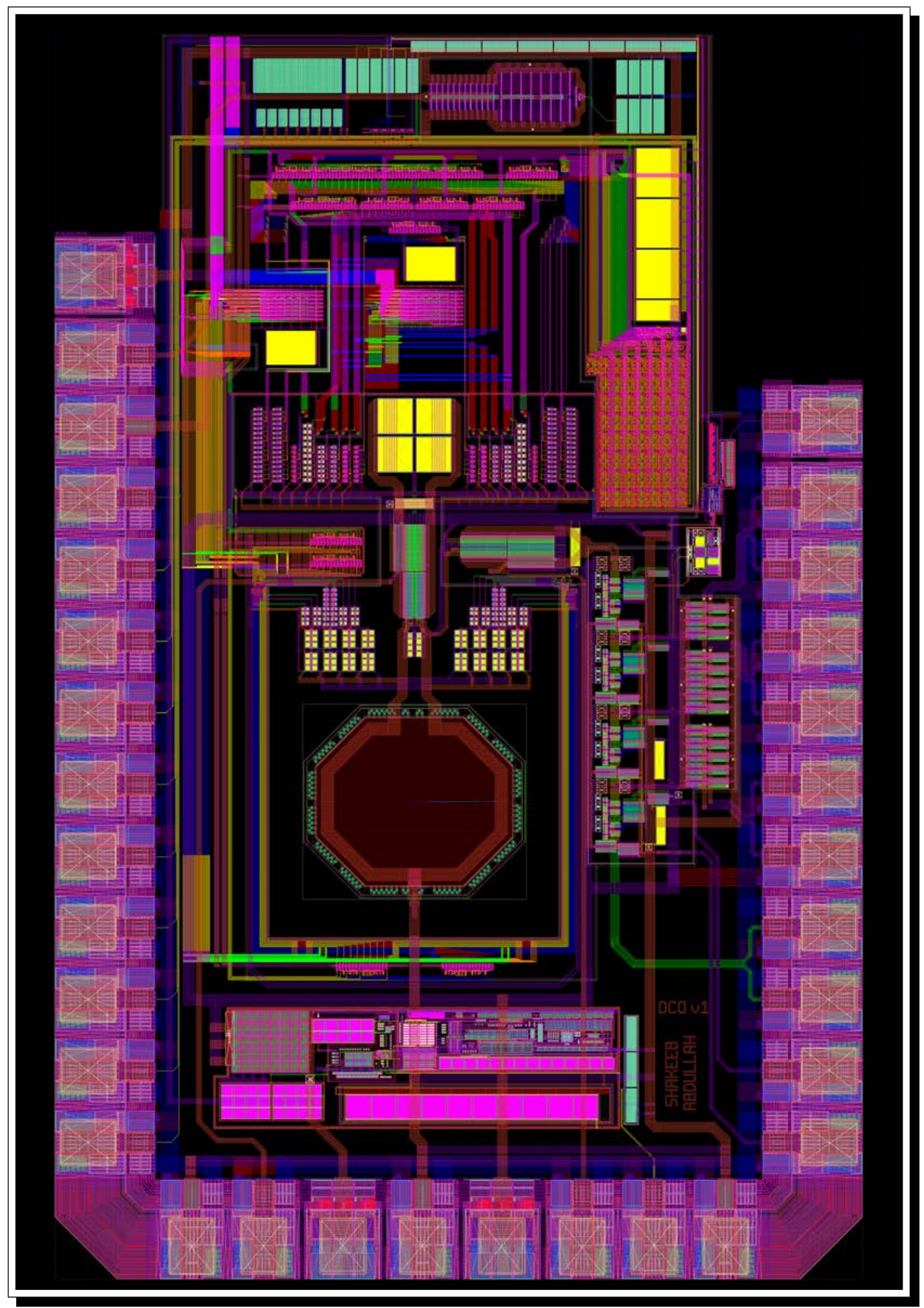

Figure 59: High resolution layout image of the DCO in Figure 58. 


\subsection{Layout of Second DCO}

A second (stripped down) and slightly modified version of the DCO of the previous sub-chapter was also designed. This was done to assess the variations in different simulators. By comparing the results of the two variants of the DCO, it would allow one to (possibly) evaluate which simulator was superior (at least that was the intent at the time of tape-out). The layout of the second DCO is shown in Figure 60. A CML for CML characterization was also placed alongside the the second oscillator in the layout.

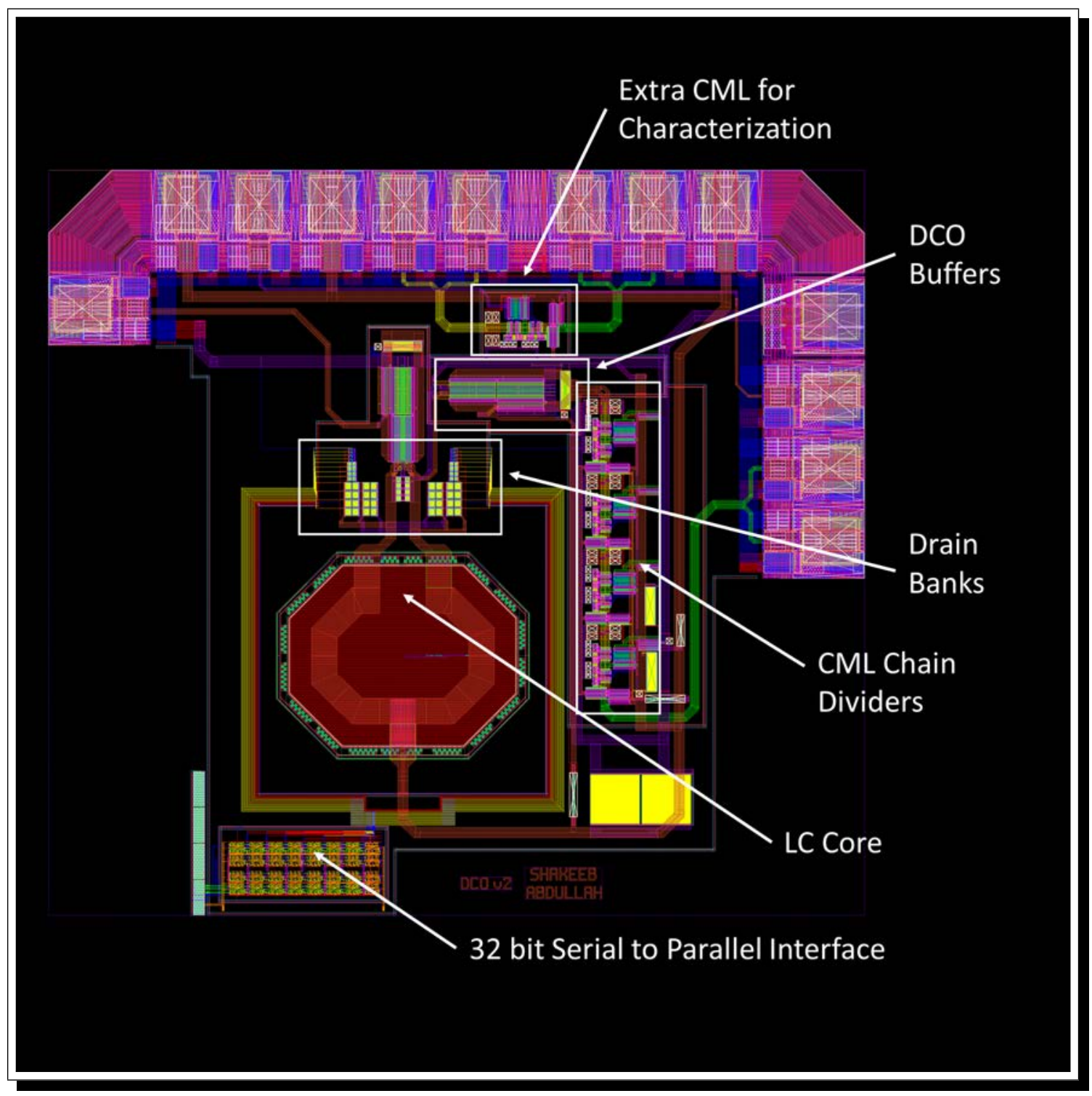

Figure 60: Layout of the second DCO. The size of the layout was $958 \mu \mathrm{m} \times 871 \mu \mathrm{m}$. 


\subsection{Full Chip Layout for Tape-out}

The entire chip layout for tape-out is shown in Figure 61 with major compartments labelled. An unlabelled version with higher graphic resolution (for clarity) is shown in Figure 62. The chip contains a full ADPLL loop consisting of a TDC (produced by ADPLL team member Tony Wang) and an in house Microsemi (Microchip) Corporation digital loop filter (designed by Krste Mitric). The chip also contains the two variations of the DCO and a CML for characterization. The chip contains 104 pads. The final dimension of the chip was $1 \mathrm{~mm}$ (width) by $4 \mathrm{~mm}$ (length).

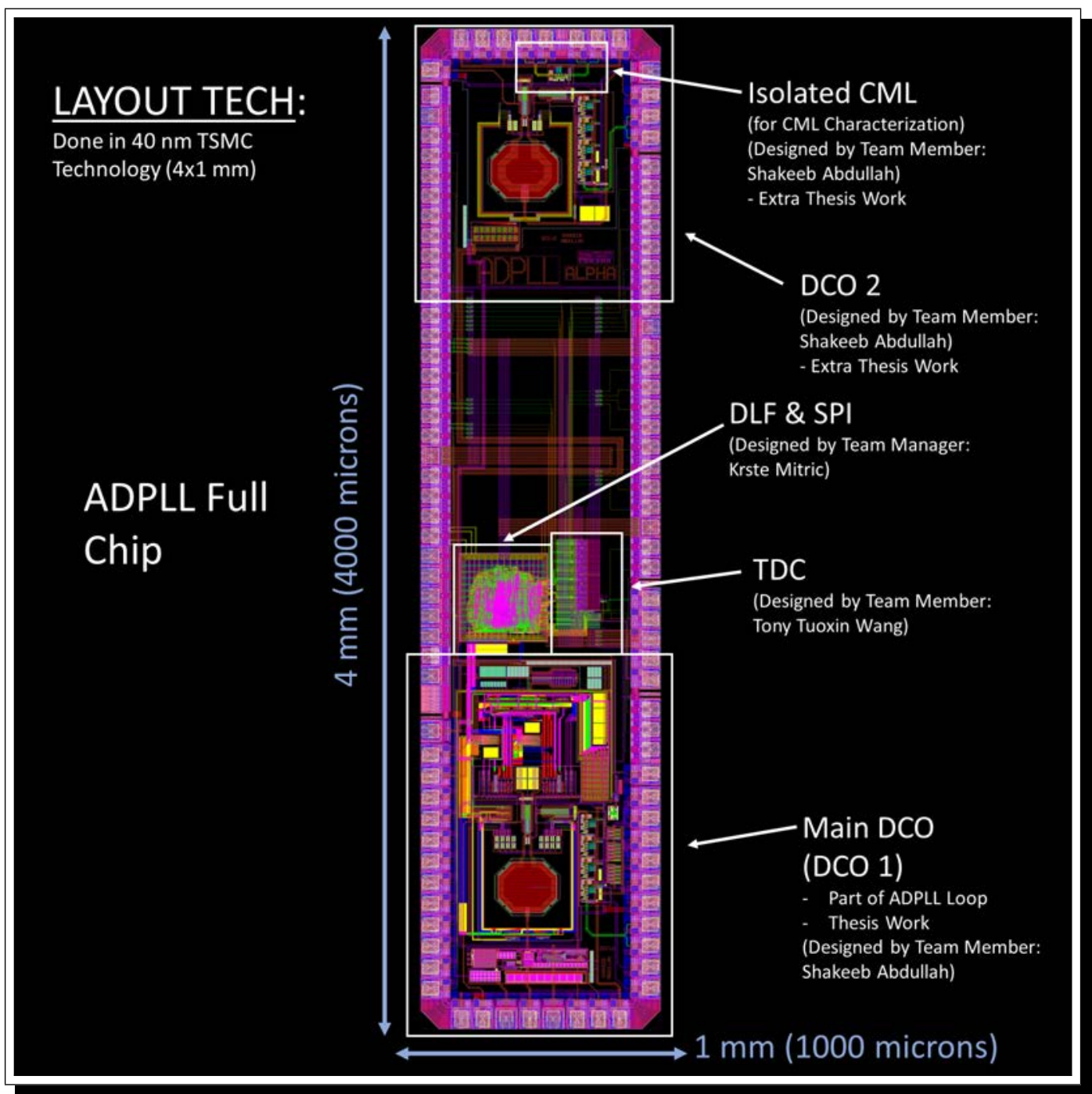

Figure 61: ADPLL full chip layout. The chip includes two DCOs, a TDC, a DLF, and an isolated CML. 


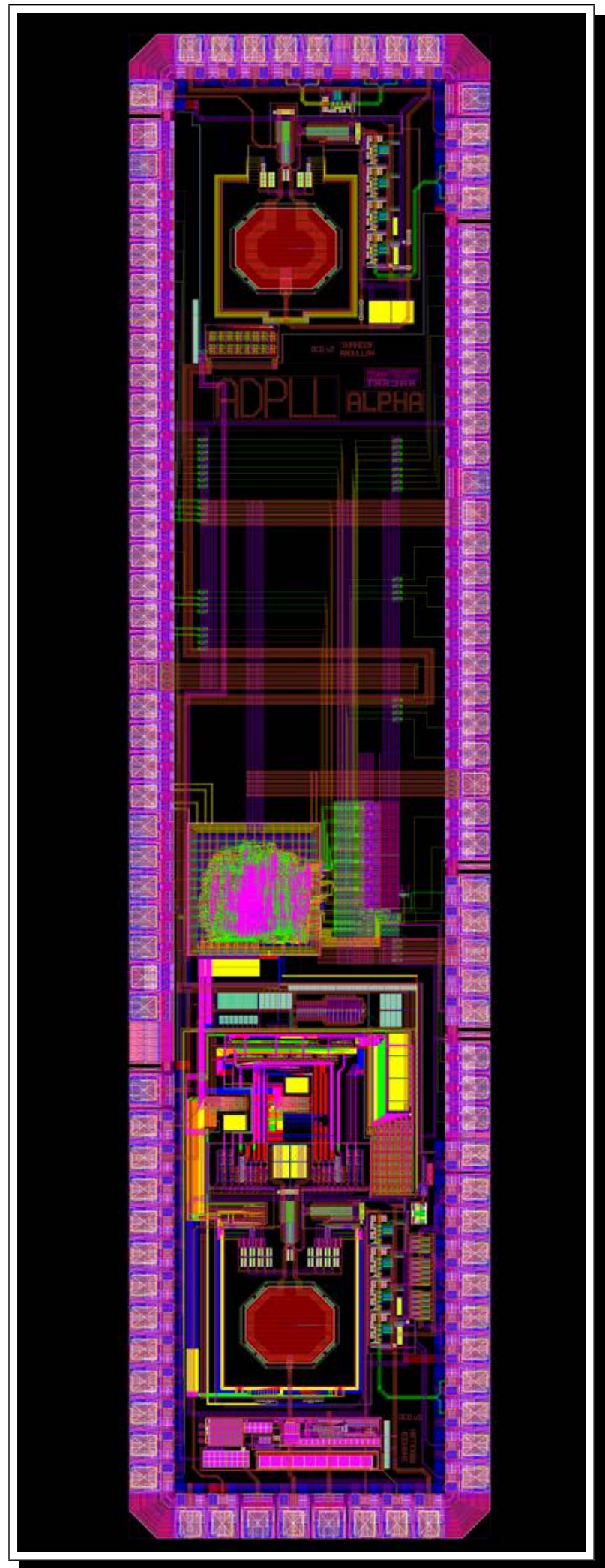

Figure 62: Final version of the chip for tape-out. (***Note: The metal, poly, and oxide dummy fills are not shown for clarity.) 
Only one tape-out of the above layout (Figure 62) was permitted, so it was of utmost pertinent that everything on the layout and all its post layout simulations were correct.

\subsection{Conclusion}

This chapter looked at the layout design of the DCO designed for this thesis. It looked at the layout design of the DCO Core, Digital Banks, Core Buffers and CML dividers. It also looked at the layout design of the digital circuitry, the buffers connecting to the bonding pads, and the overall layout. Only one tape-out of the layout was permitted. 


\section{CHAPTER 7: SIMULATION RESULTS}

This chapter will discuss the simulated results of the extracted (post-layout) simulations from the layouts designed in the previous Chapter; along with any other simulated results that were needed for proper functioning and implementation of the DCO. All simulations presented in this Chapter were done under TT (typical-typical) conditions. Unless otherwise specified, all simulations were done using EMX (electromagnetic simulator tool) [45] with RLC (resistance-capacitance-inductance) extractions.

\subsection{Transient Simulations of DCO}

The transient simulation of the DCO (in Figure 47) when all the banks are off is shown in Figure 63. The frequency of the transient simulation was $7.14 \mathrm{GHz}$.

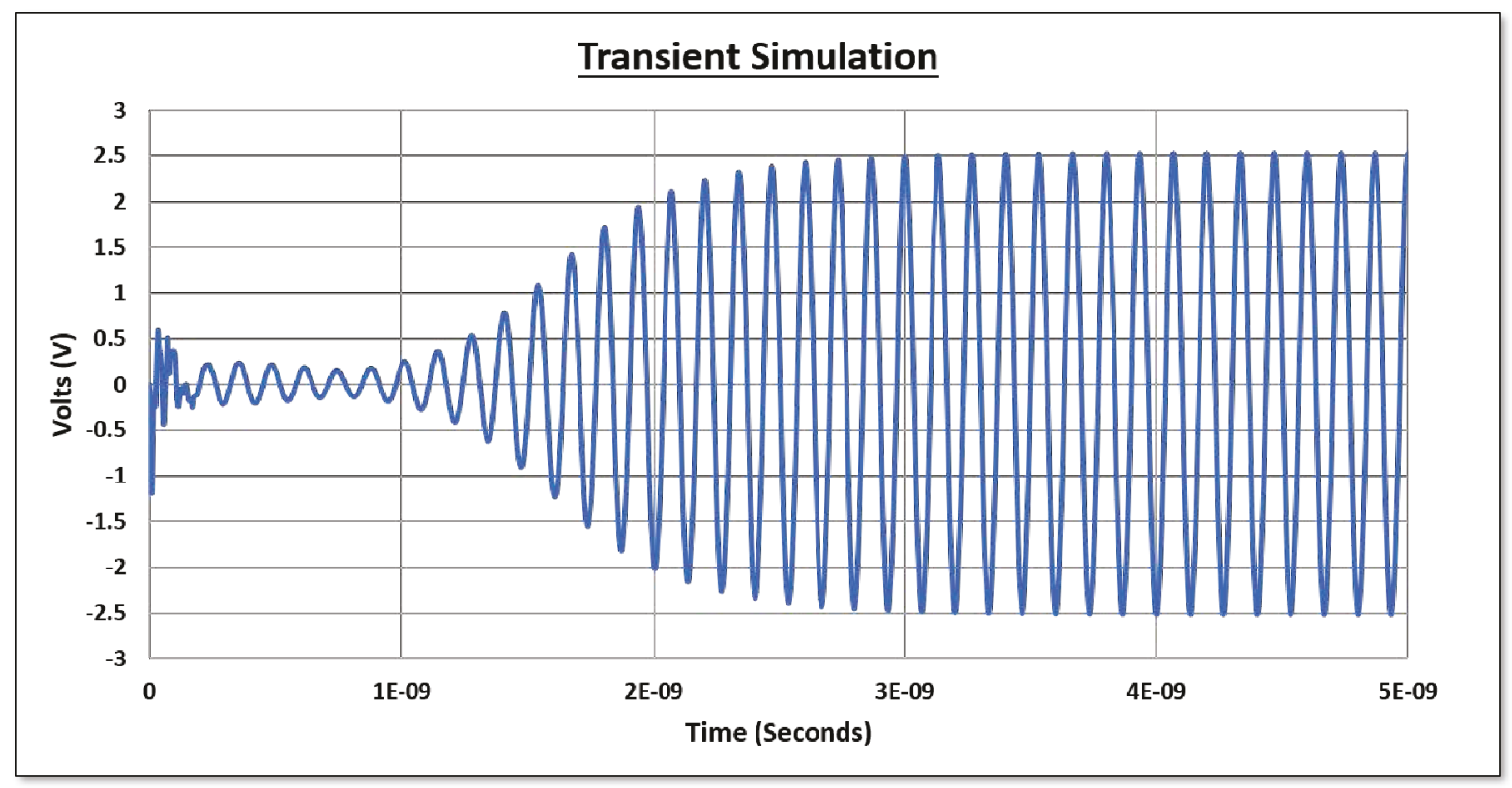

Figure 63: Post layout transient simulations of DCO with all banks off. The frequency was $7.14 \mathrm{GHz}$.

The transient simulation of the DCO when all the banks are on (including all bank sets) is shown in Figure 64. The frequency of the transient simulation was $5.74 \mathrm{GHz}$. 


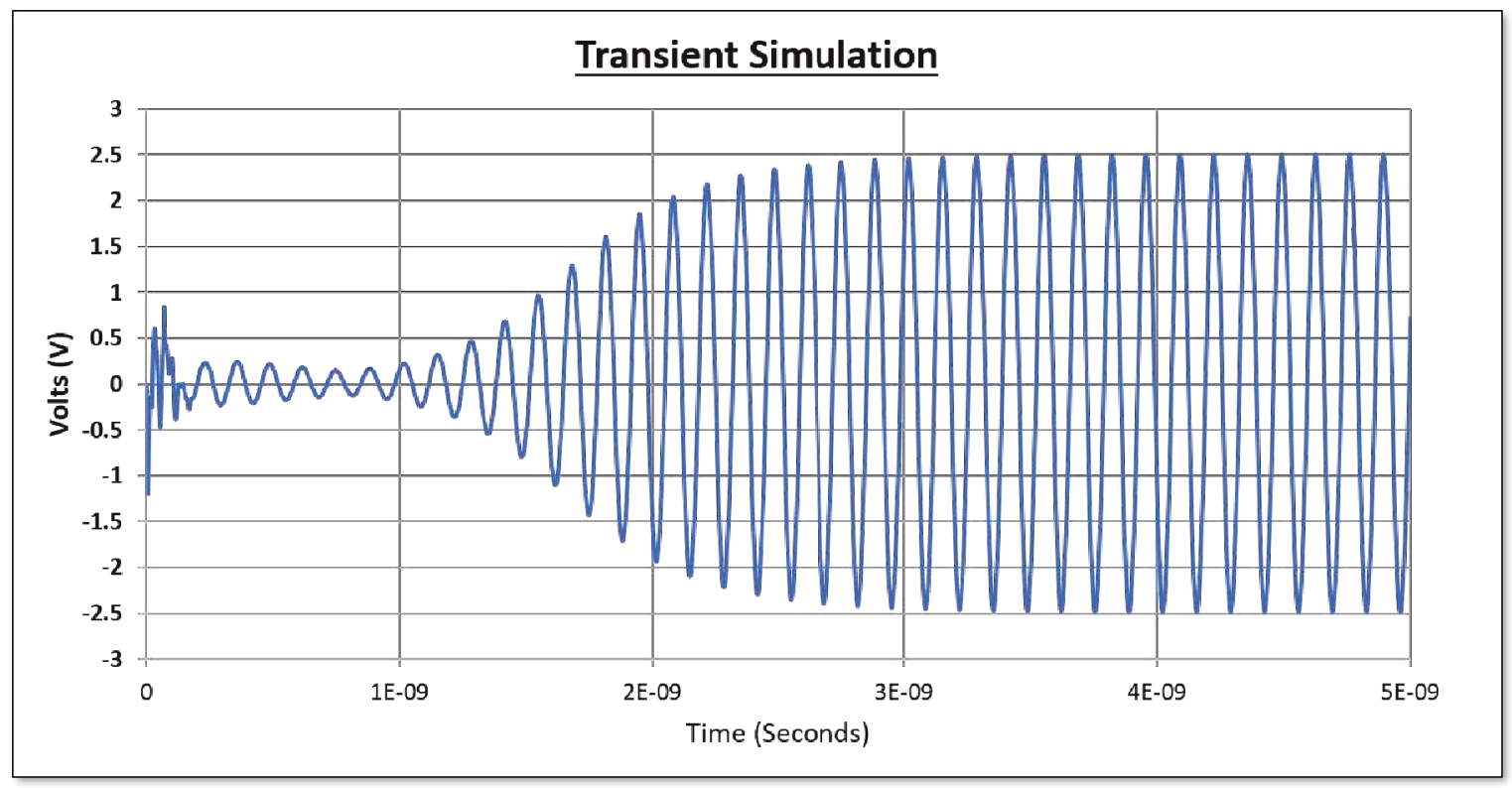

Figure 64: Post layout transient simulations of DCO with all banks on. The frequency was $5.74 \mathrm{GHz}$.

\subsection{Frequency Simulations of DCO}

The simulation engine used to acquire the frequency response was Harmonic Balance (HB). HB was used instead of Periodic Steady State (PSS) because PSS did not work with the heavy layout extractions of the DCO, and when it did work it was too slow.

\subsubsection{Frequency simulations of Bank set 1}

The frequency step simulations of Bank set 1 is summarized and displayed in Figure 65 .

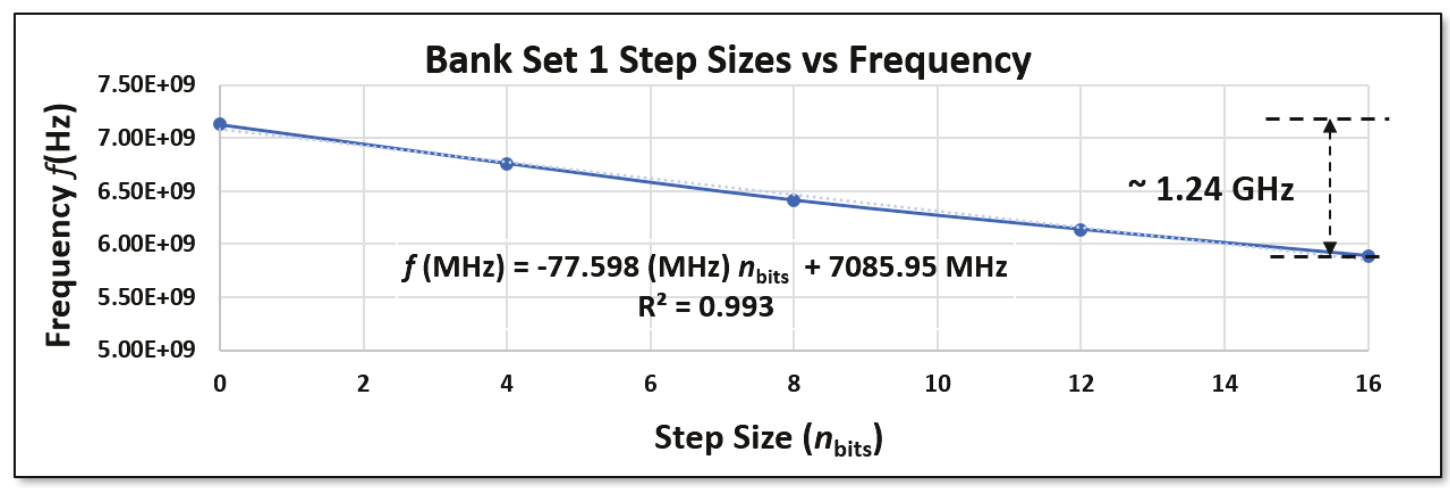

Figure 65: Frequency simulations of stepping through Bank set 1.

The total frequency span of Bank set 1 was $1.24 \mathrm{GHz}$ with an average frequency step size 
of:

$$
\operatorname{Bank}_{1}(\text { avg. })=\frac{1.24 \mathrm{GHz}}{16} \approx 77.5 \mathrm{MHz}
$$

This can be seen by the slope of the interpolating software with $m=-77.598 \mathrm{MHz}$.

\subsubsection{Frequency simulations of Bank 2}

The frequency step simulations of Bank set 2 is summarized and displayed in Figure 66.

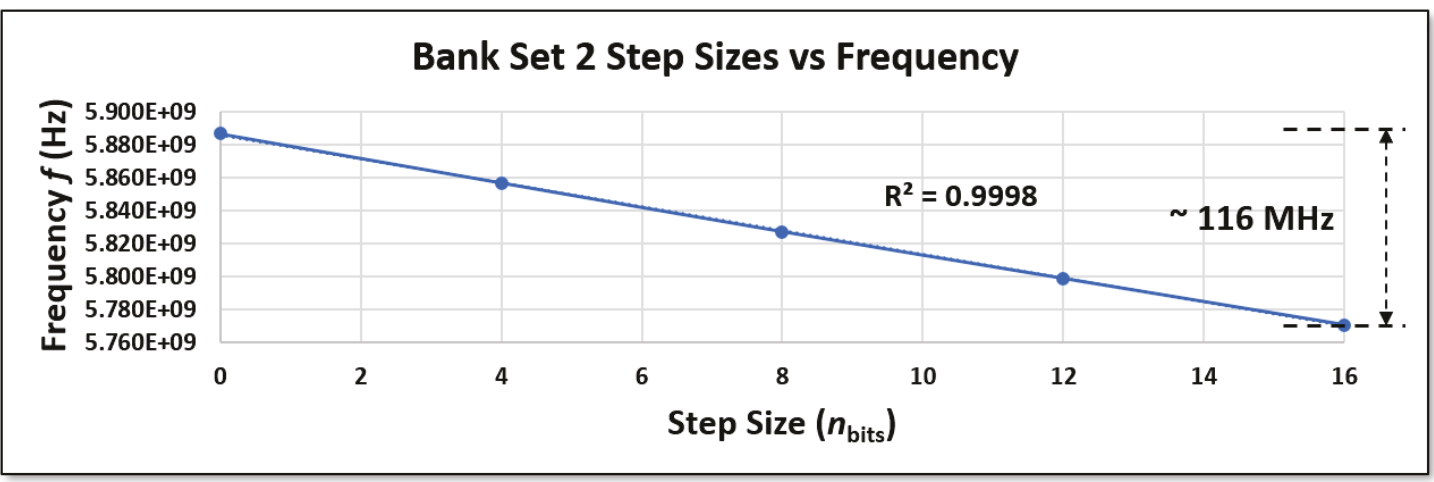

Figure 66: Frequency simulations of stepping through Bank set 2.

The total frequency span of Bank set 2 was $116 \mathrm{MHz}$ with an average frequency step size of $7.25 \mathrm{MHz}$.

\subsubsection{Frequency simulations of Bank Set 3}

The frequency step simulations of Bank Set 3 is summarized and displayed in Figure 67.

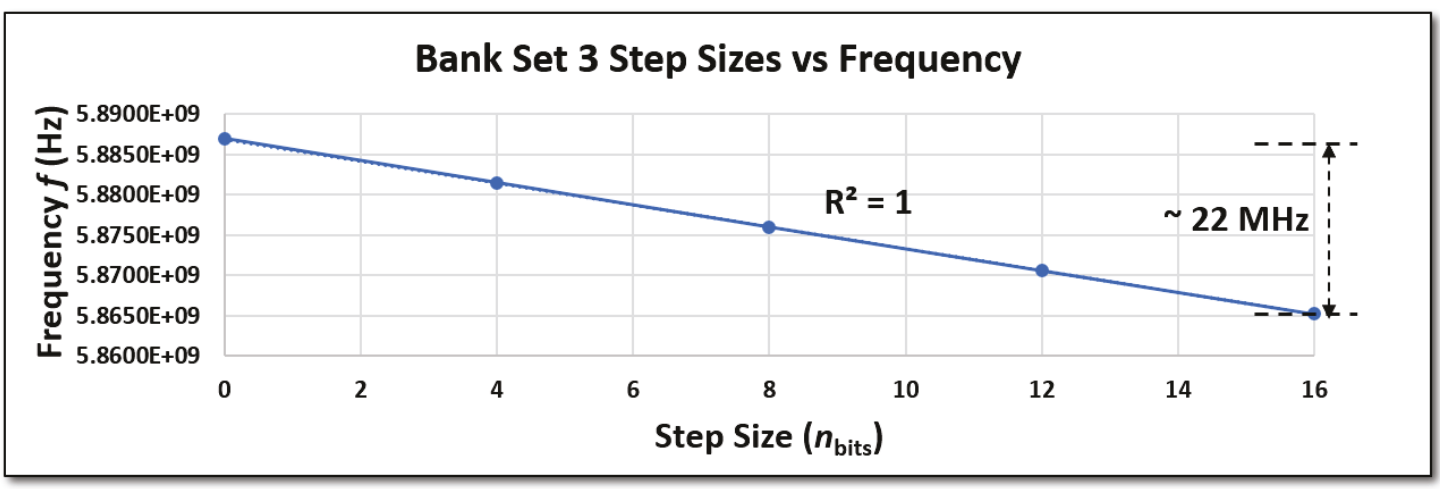

Figure 67: Frequency simulations of stepping through Bank set 3.

The total frequency span of Bank set 3 was $22 \mathrm{MHz}$ with an average frequency step size of $1.4 \mathrm{MHz}$. 


\subsubsection{Frequency simulations of Bank Set 4}

The frequency step simulations of Bank set 4 is summarized and displayed in Figure 68.

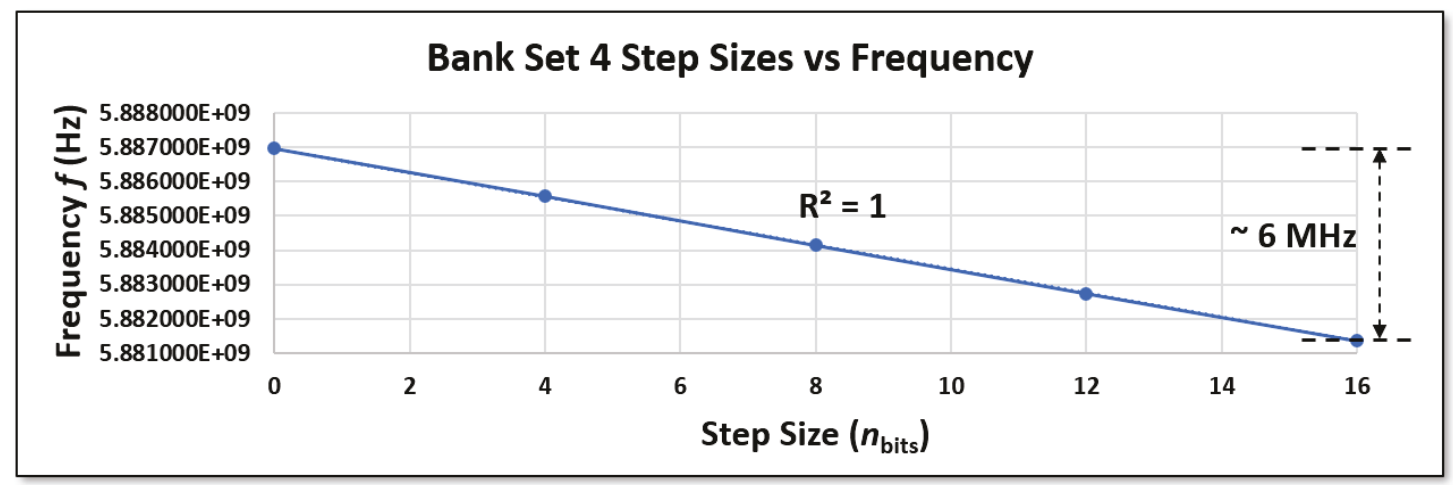

Figure 68: Frequency simulations of stepping through Bank set 4 .

The total frequency span of Bank set 4 was $6 \mathrm{MHz}$ with an average frequency step size of $375 \mathrm{kHz}$.

\subsubsection{Frequency simulations of Bank Set 5}

The frequency step simulations of Bank set 5 is summarized and displayed in Figure 69.

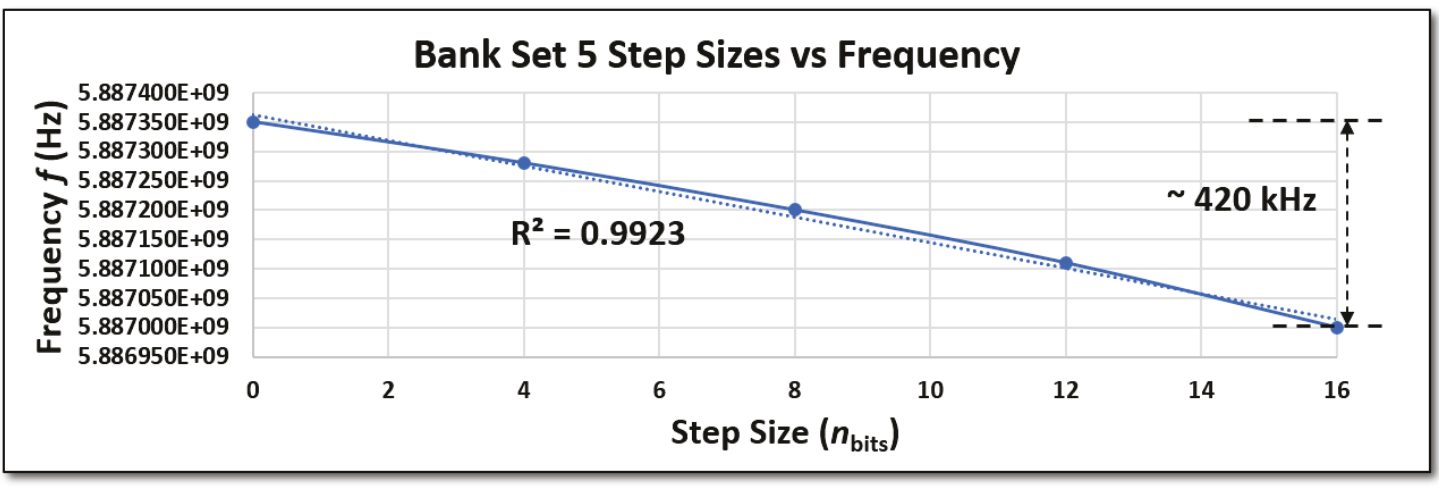

Figure 69: Frequency simulations of stepping through Bank set 5.

The total frequency span of Bank set 5 was $420 \mathrm{kHz}$ with an average frequency step size of $26.3 \mathrm{kHz}$.

\subsubsection{Frequency simulations of Bank Set 6}

The frequency step simulations of Bank Set 6 is summarized and displayed in Figure 70 . 


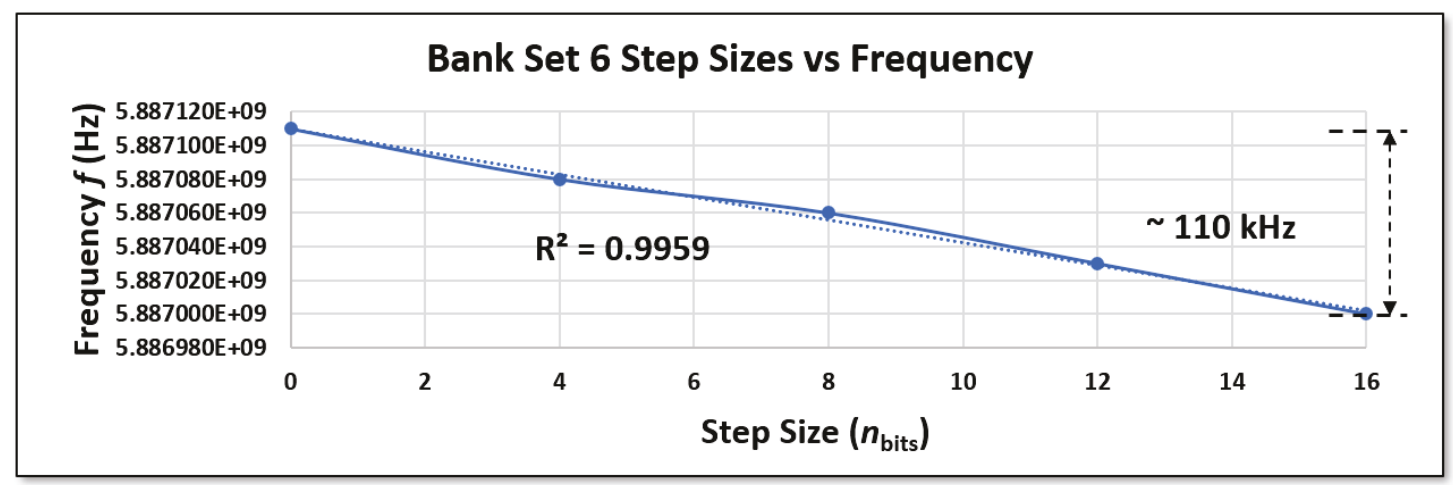

Figure 70: Frequency simulations of stepping through Bank set 6 .

The total frequency span of Bank set 6 was $110 \mathrm{kHz}$ with an average frequency step size of $6.9 \mathrm{kHz}$.

\subsubsection{Frequency simulations of Bank set 7 to Bank set 11}

Getting good simulation results for Bank set 7 to Bank set 11 was challenging due to software computational limits. An example is shown in Figure 71 for Bank set 7 . To get around this issue, an average of large amount of banks was taken for Bank set 7 to Bank set 11 and is summarized in the next section.

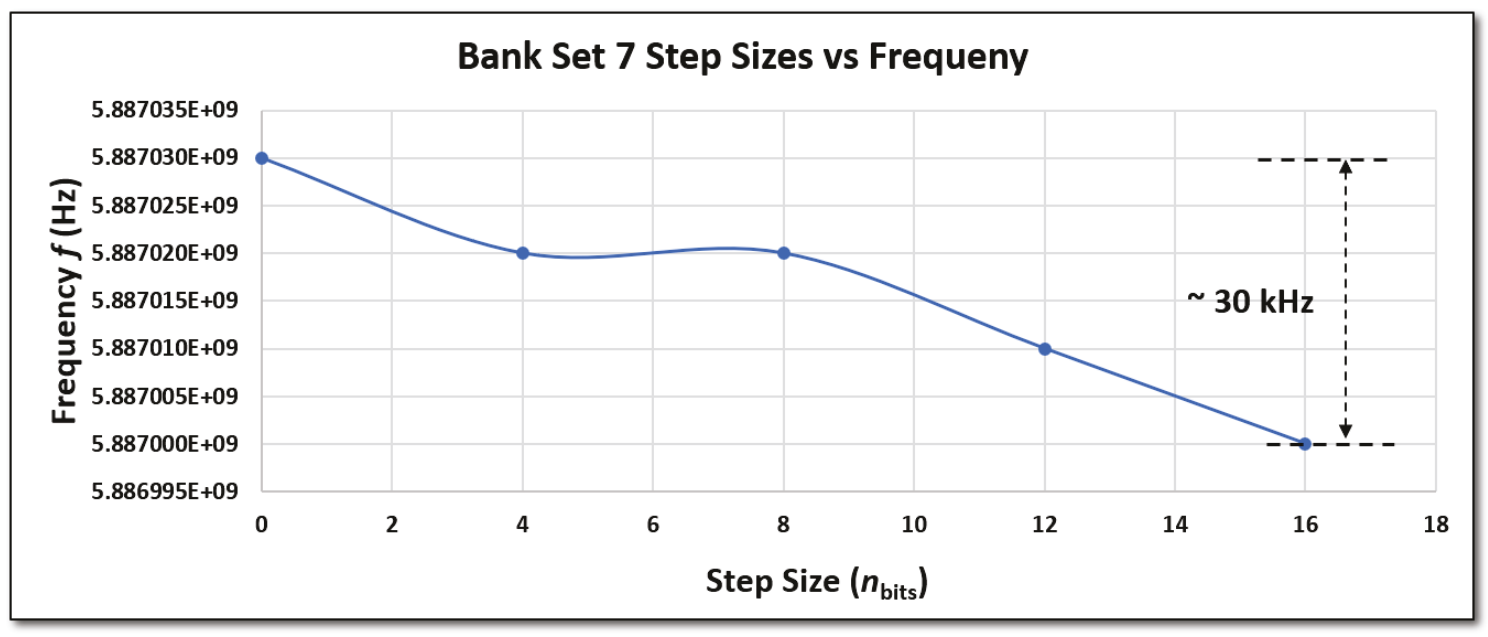

Figure 71: Frequency simulations of stepping through bank set 7 .

\subsubsection{Summary of frequencies \& step sizes of the Bank sets}

A table summarizing the average frequency steps of each bank sets is shown in Table 3 . 
TABLE 3: Simulated Average Frequency Step Size for Each Bank Set.

\begin{tabular}{|c|c|c|}
\hline Bank Number & Number of Bank Units & Simulated Frequency Step Size \\
\hline Bank Set 1 & 16 & $77.5 \mathrm{MHz}$ \\
\hline Bank Set 2 & 16 & $7.27 \mathrm{MHz}$ \\
\hline Bank Set 3 & 16 & $1.36 \mathrm{MHz}$ \\
\hline Bank Set 4 & 16 & $351.25 \mathrm{kHz}$ \\
\hline Bank Set 5 & 20 & $21 \mathrm{kHz}$ \\
\hline Bank Set 6 & 16 & $6.875 \mathrm{kHz}$ \\
\hline Bank Set 7 & 16 & $1.875 \mathrm{kHz}$ \\
\hline Bank Set 8 & 16 & $3.125 \mathrm{kHz}$ \\
\hline Bank Set 9 & 16 & $625 \mathrm{~Hz}$ \\
\hline Bank Set 10 & 16 & $625 \mathrm{~Hz}$ \\
\hline Bank Set 11 & 64 & $156.25 \mathrm{~Hz}$ \\
\hline
\end{tabular}

\subsection{Phase Noise Simulations of DCO}

All phase noise simulations were done using the harmonic balance engine (like the simulations for the frequency responses of the DCO). The digital circuits were omitted when simulating phase noise due to computational limits; since the simulations could not converge. To emulate the digital stimuli, the banks were either pulled up to the digital voltage source (VDD) or pulled down to ground using a pull-up/pull-down resistor (attached to the gate of the bank transistor switch).

The phase noise simulation at $1 \mathrm{MHz}$ offset (from carrier frequemcy of the DCO) when all the banks are turned off (at $7.14 \mathrm{GHz}$ ) is shown in Figure 72 . 


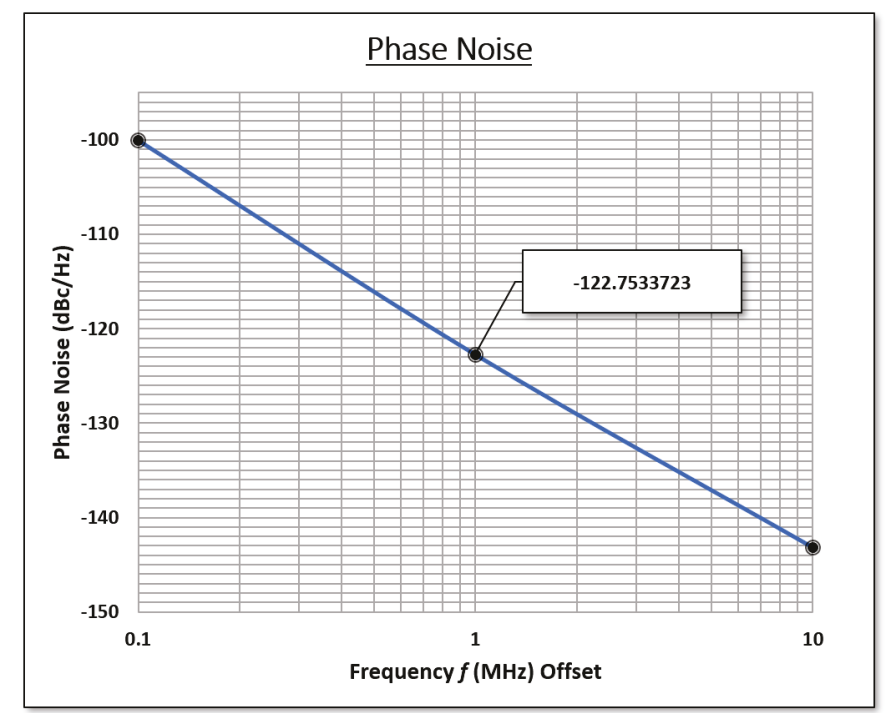

Figure 72: The simulated phase noise of the oscillator when all banks are turned off.

The simulated phase noise of the oscillator when all banks were turned off was -122.8 $\mathrm{dBc} / \mathrm{Hz}$ at $1 \mathrm{MHz}$ offset.

The phase noise simulation at $1 \mathrm{MHz}$ offset when all banks were on (at $5.74 \mathrm{GHz}$ ) is shown in Figure 73.

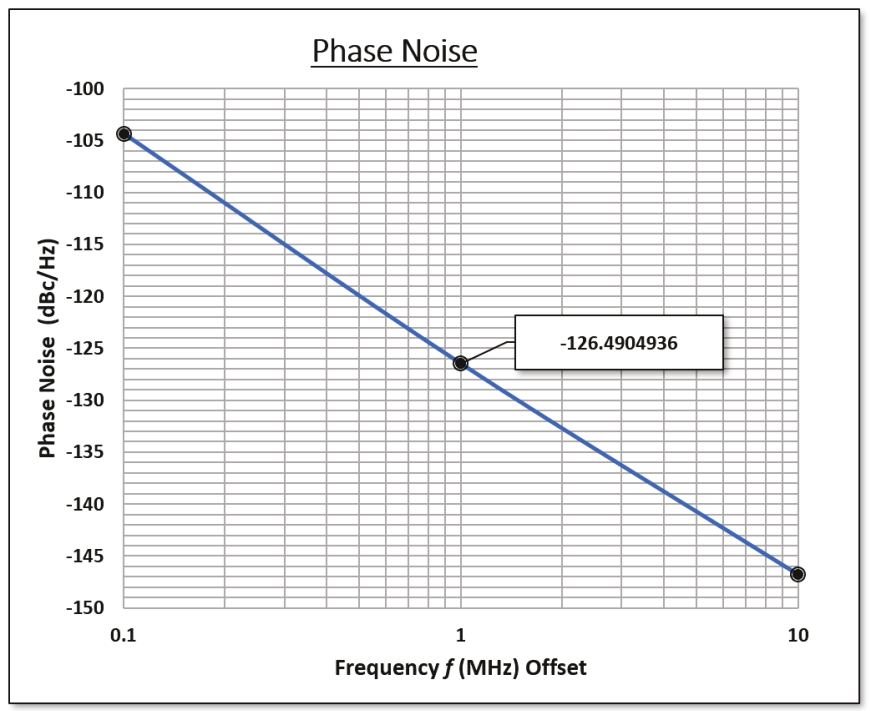

Figure 73: The simulated phase noise of the oscillator when all banks are turned on.

The phase noise at $1 \mathrm{MHz}$ offset when all banks were turned on was $-126.5 \mathrm{dBc} / \mathrm{Hz}$. The phase noise simulation over the entire frequency spectrum of the DCO is shown in Figure 
74 and ranges between $-122.8 \mathrm{dBc} / \mathrm{Hz}$ to $-126.5 \mathrm{dBc} / \mathrm{Hz}$ at $1 \mathrm{MHz}$ offset. The equivalent phase noise at $740 \mathrm{MHz}$ carrier is also shown on the plot in Figure 74.

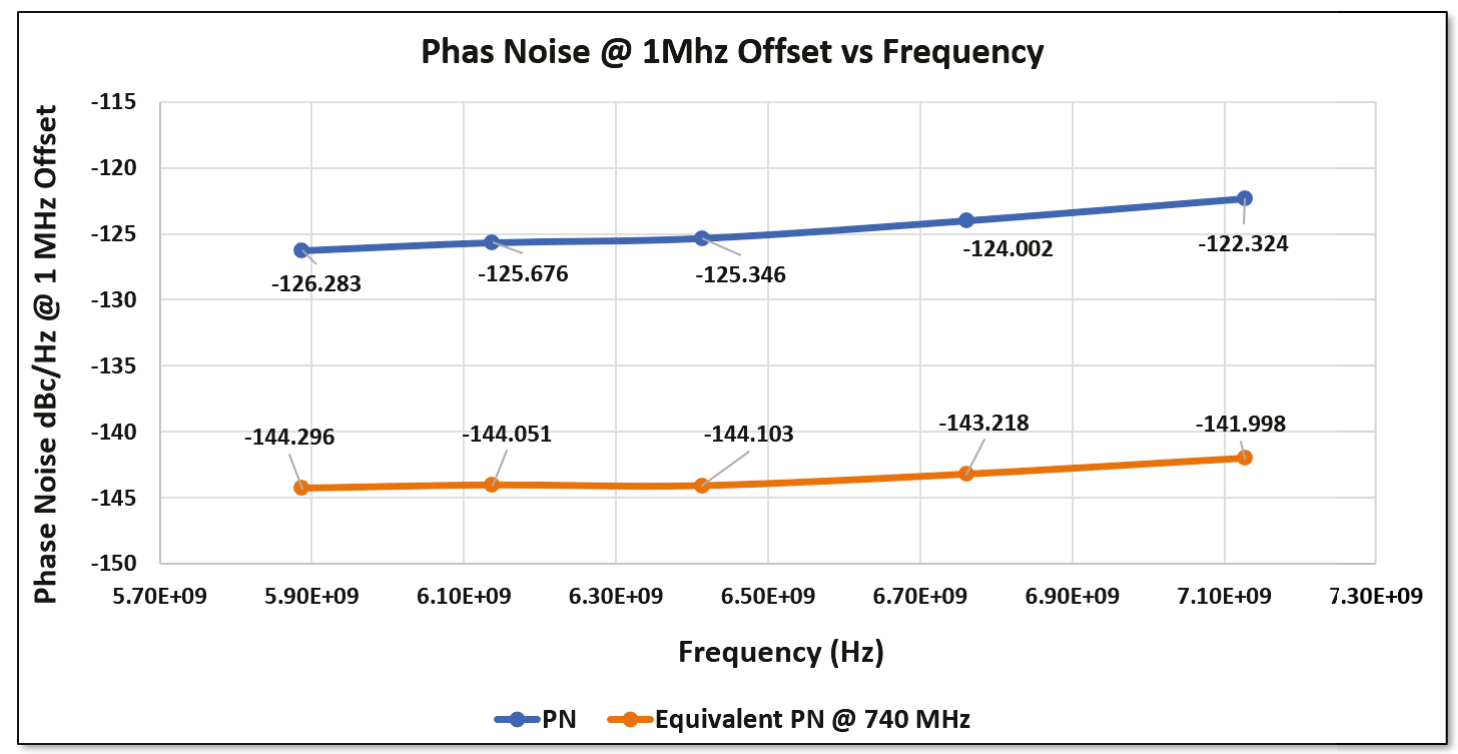

Figure 74: The simulated phase noise over the entire frequency span of the DCO at $1 \mathrm{MHz}$ offset.

\subsection{Power vs Phase Noise Simulations}

The simulated phase noise performance vs current (and hence power) when all banks are off is shown in Figure 75.

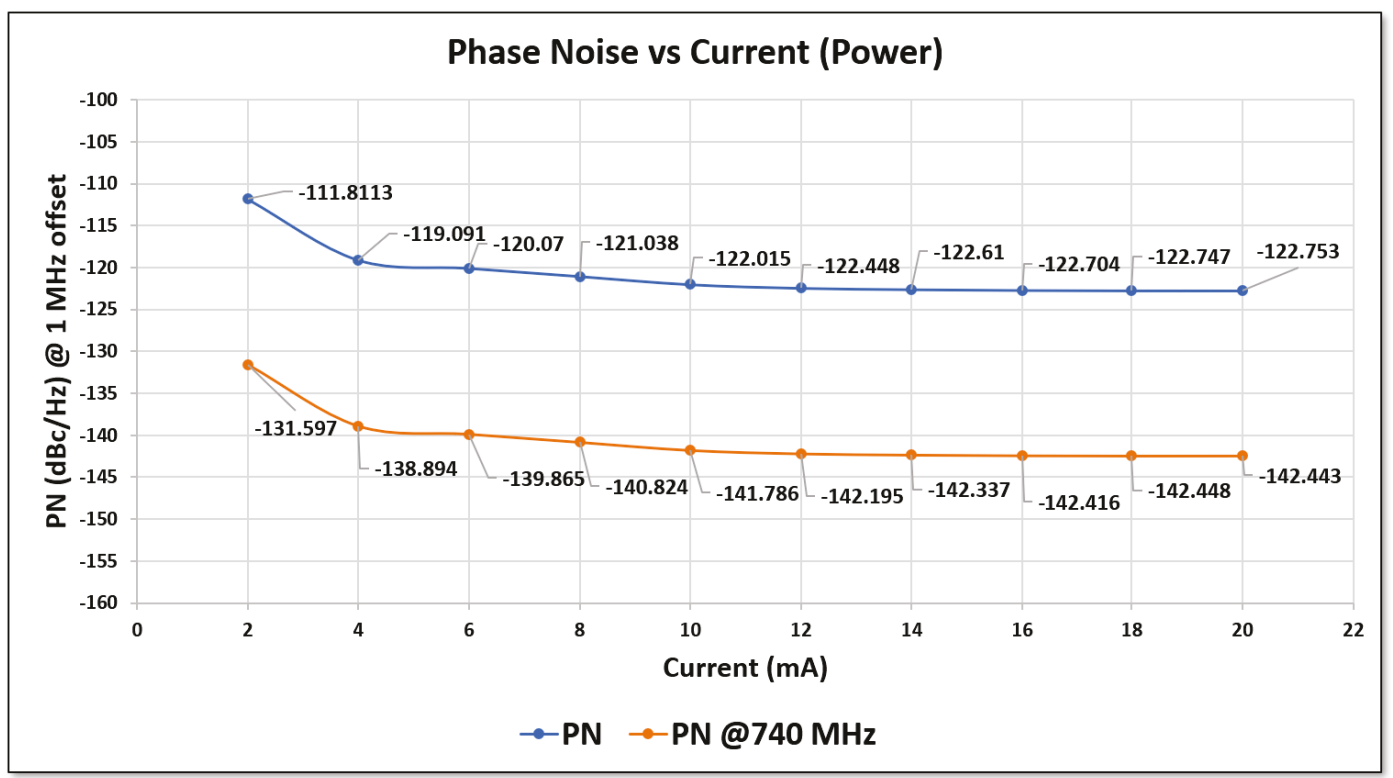

Figure 75: The simulated phase noise performance of DCO vs current (at $1 \mathrm{MHz}$ offset). 
From Figure 75, as the current is increased, the phase noise performance improved as well before reaching diminishing returns at around 10-14 mA.

\subsubsection{Effects on PN due to CML dividers}

It was crucial that the impact of the CML dividers to the degradation of the phase noise of the oscillator was minimal. Phase noise simulation at the divided frequency of the last CML divider is shown in Figure 76. The expected phase noise for a 7.14 Ghz carrier that was divided by four CMLs (divide by 16 since $2^{4}$ ) at a divided carrier frequency of $446 \mathrm{MHz}$ and $1 \mathrm{MHz}$ offset should be $-146.4 \mathrm{dBc} / \mathrm{Hz}$ :

$\mathrm{PN}(446 \mathrm{MHz}$ at $1 \mathrm{MHz}$ offset $)=-122.324\left(\frac{\mathrm{dBc}}{\mathrm{Hz}}\right)-20 \cdot \log _{10}\left(\frac{7.14 \mathrm{GHz}}{446 \mathrm{MHz}}\right) \approx-146.4\left(\frac{\mathrm{dBc}}{\mathrm{Hz}}\right)$

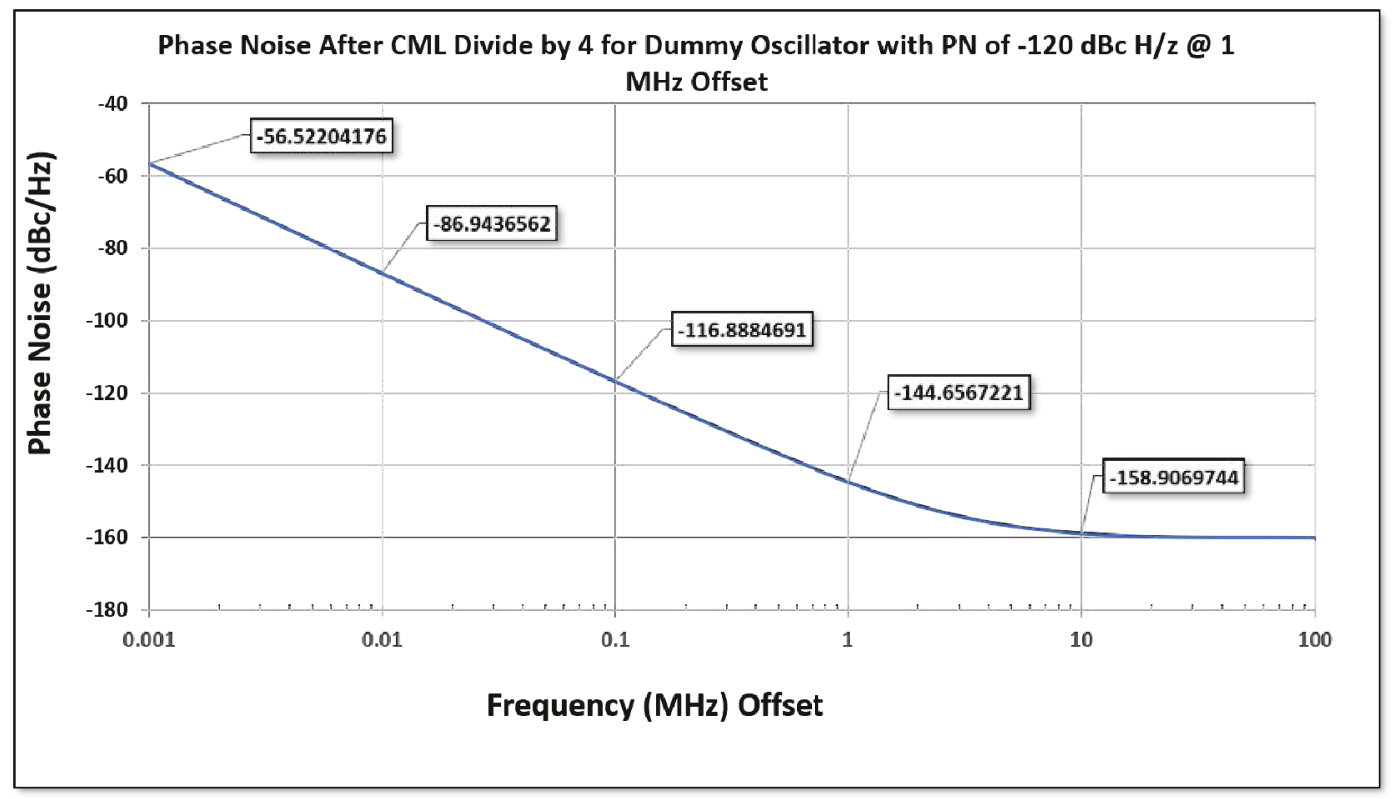

Figure 76: The phase noise performance at the output of four CML dividers in series.

The simulated phase noise result after division from the CMLs (at $1 \mathrm{MHz}$ offset) was -144.6 $\mathrm{dBc} / \mathrm{Hz}$. This suggests that the CMLs degraded the oscillator phase noise performance by about $2 \mathrm{~dB}$ :

$$
(-146.4) \mathrm{dBc} / \mathrm{Hz}-(-144.7 \mathrm{dBc} / \mathrm{Hz}) \simeq 1.7 \mathrm{~dB} \approx 2 \mathrm{~dB}
$$




\subsubsection{FoM}

The figure of merit of the oscillator for different DCO frequencies is shown in Figure 77.

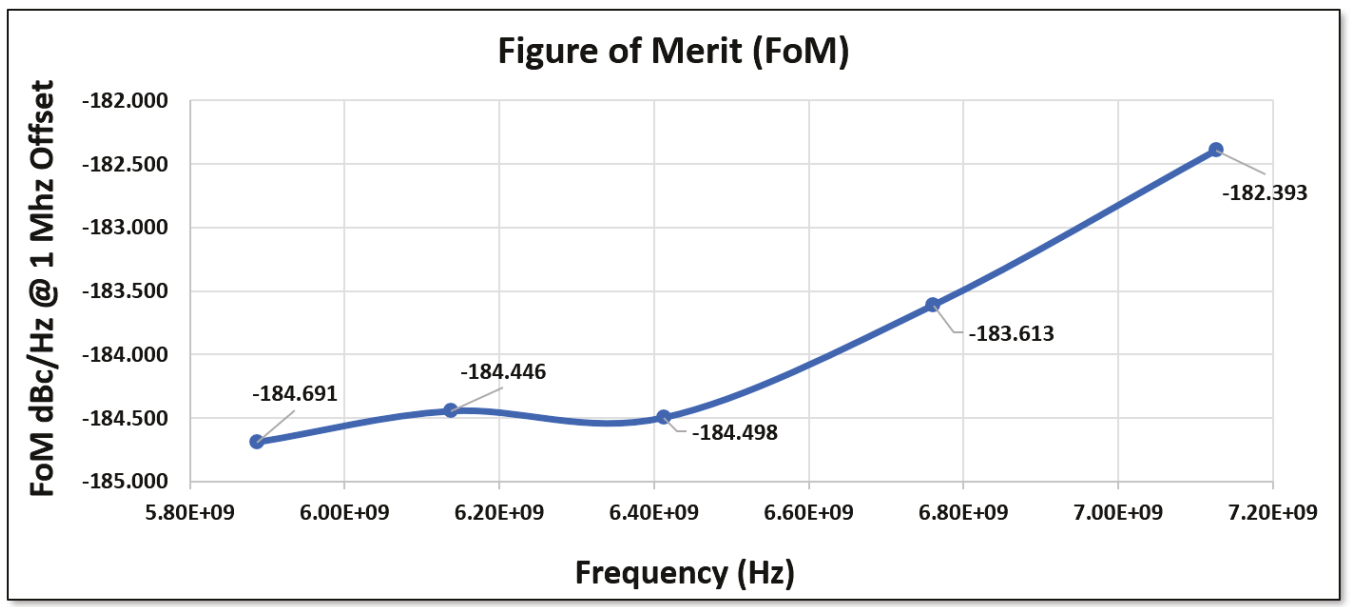

Figure 77: Figure of merit over DCO tuning range.

The simulated figure of merit results rested between $-182 \mathrm{dBc} / \mathrm{Hz}$ to $-185 \mathrm{dBc} / \mathrm{Hz}$. The figure of merit of the DCO at $7.14 \mathrm{GHz}$ (when all banks are off) for various current (power) levels is shown in Figure 78.

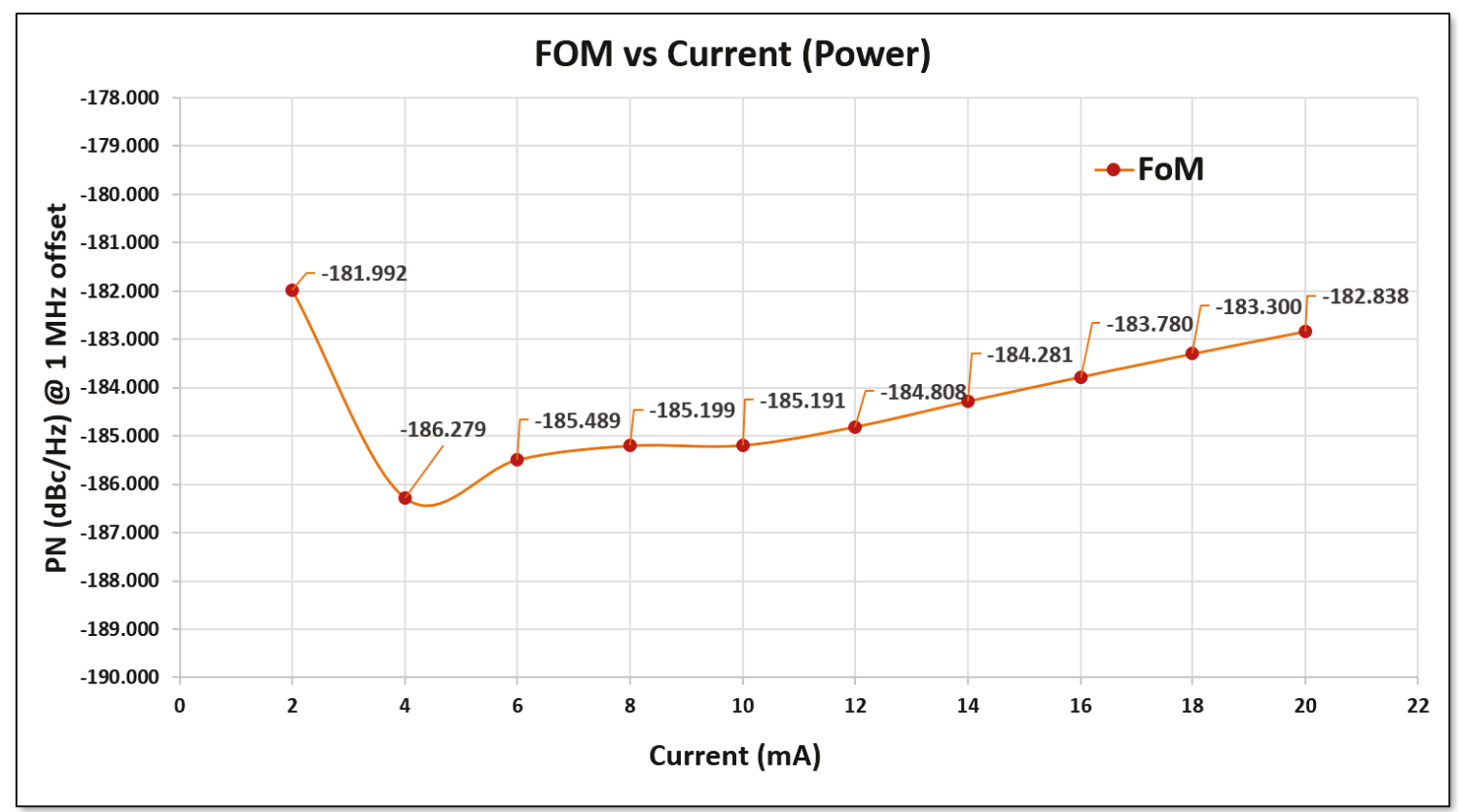

Figure 78: FoM vs current at $7.14 \mathrm{GHz}$ when all banks are off. 


\subsection{Transient Simulations}

The divided transient simulations from the outputs of the CMLs is shown in Figure 79. The transient simulation of the CML was simulated from EMX (RLC) extraction under TT conditions. The input of the CML was fed from the output of the EMX (RLC) extracted DCO.

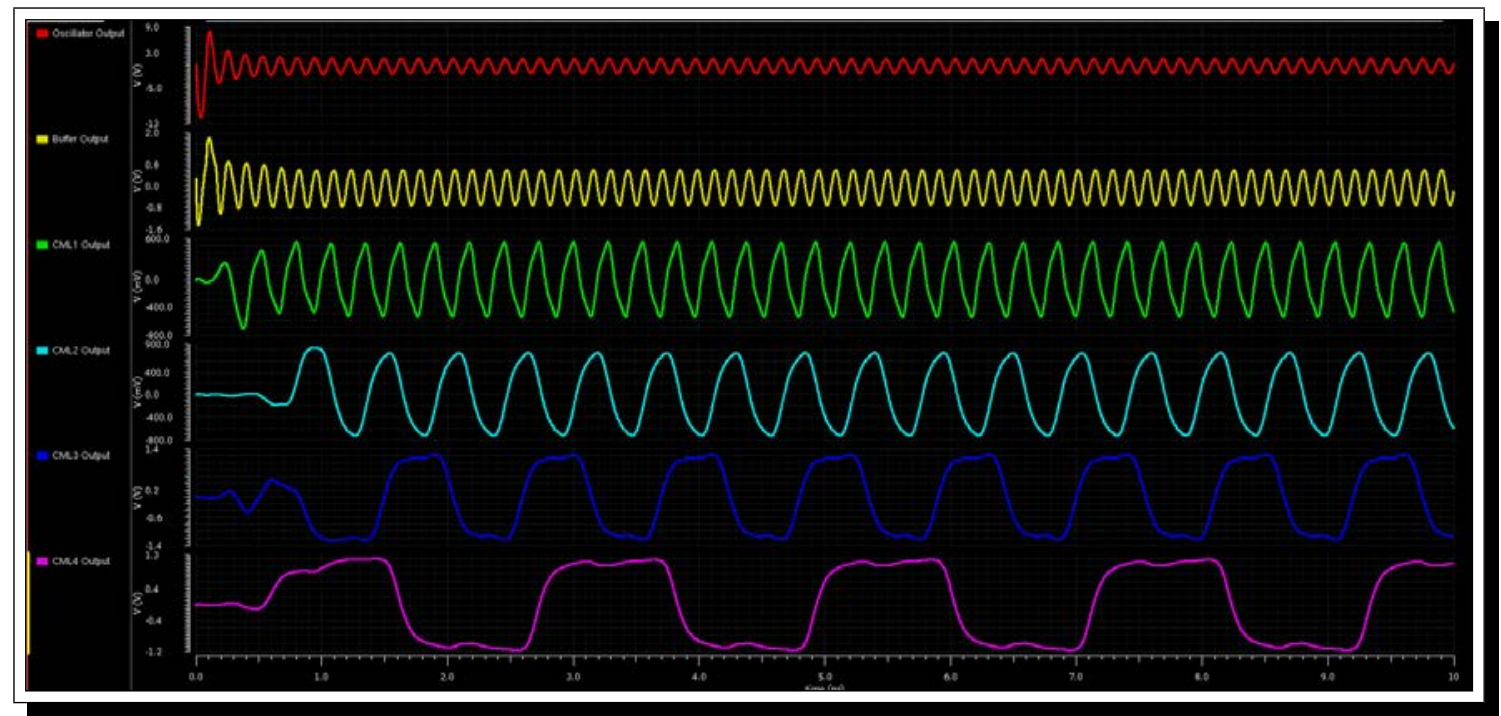

Figure 79: Transient simulation of CML divisions.

As can be observed from Figure 79, the output transient simulation of a CML takes twice the cycle of the output transient waveform of the previous CML.

\subsection{Tunning Range of Oscillator}

The full tuning range of the oscillator (including all 11 set of banks) from simulation results was $21.7 \%$ :

$$
\text { Tunning Range }(\mathrm{TR}) \%=100 \cdot\left[\frac{2(7.14 \mathrm{GHz})-2(5.74 \mathrm{GHz})}{7.14 \mathrm{GHz}+5.74 \mathrm{GHz}}\right] \approx 21.7 \%
$$

Then tuning range of only bank set 1 from simulation (with all other bank sets off) was $19.0 \%$ :

Tunning Range $(\mathrm{TR}) \%=100 \cdot\left[\frac{2(7.14 \mathrm{GHz})-2(5.90 \mathrm{GHz})}{7.14 \mathrm{GHz}+5.90 \mathrm{GHz}}\right] \approx 19.0 \%$ 


\subsection{Digital Clock Simulation of Ring Oscillator}

The digital clock simulation of the divided ring oscillator output is shown in Figure 80. The simulations were done using Calibre $\mathrm{RC}(\mathrm{C}+\mathrm{CC})$ extractions [46].

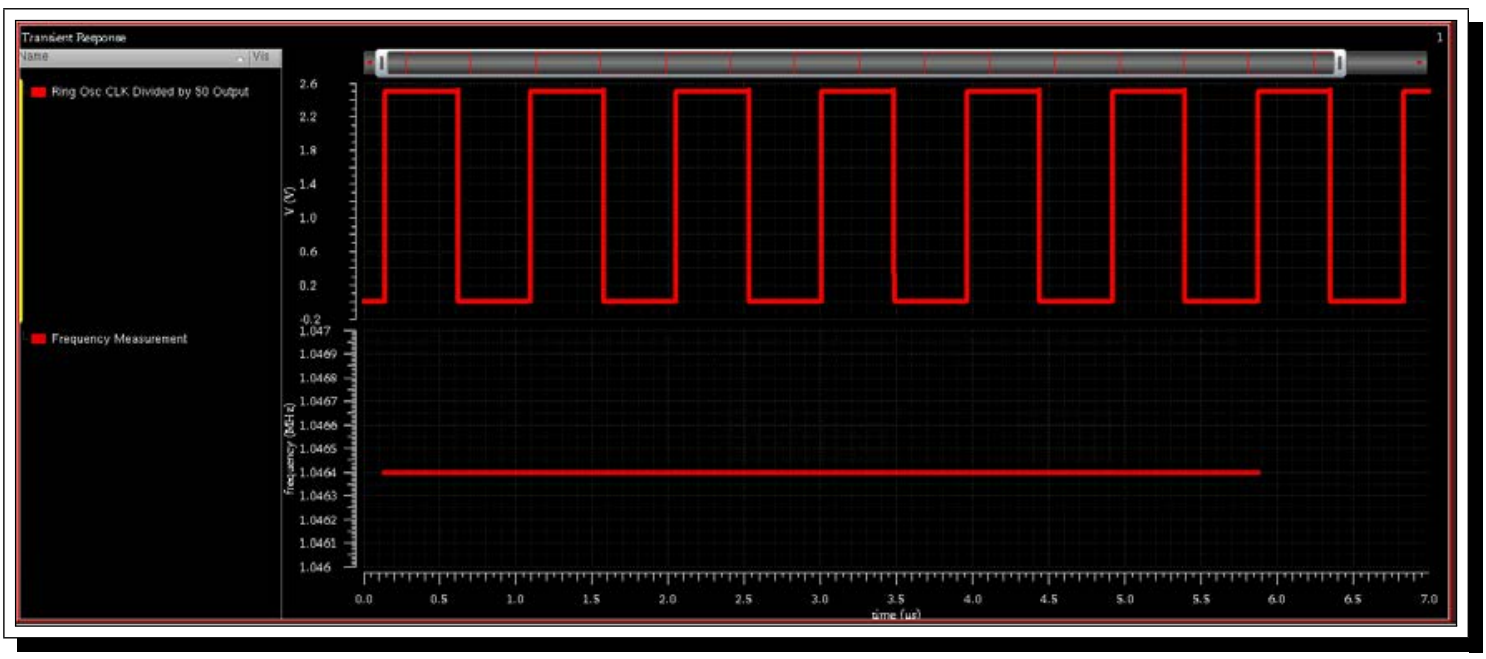

Figure 80: Transient simulation of the ring oscillator frequency divided by 50 .

\subsection{Conclusion}

This chapter looked at the post-layout simulations of the DCO designed in the previous chapters. It looked at the transient simulation response of the DCO, the frequency step responses of the DCO banks, and the simulated phase noise performance of the DCO. It also looked at the phase noise performance vs current (power) of the DCO, and the effects the CMLs had on phase noise degradation. The chapter then looked at the FoM over various power and frequencies of the DCO. The chapter also looked at the CML transient simulations, the simulated tuning range of the oscillator, and the transient simulation of the built in digital ring oscillator. 


\section{CHAPTER 8: TEST SETUP}

This chapter will discuss the test set-up of the DCO starting from the die, bonding and packaging, to the type of equipment that was used for measuring and verifying the DCO performance (including the procedures and steps in between).

\subsection{The DCO Test Chip Die}

The fabricated dies of the DCO (part of the full ADPLL chip) is shown in Figure 81 and a few examples of zoomed in views (underneath the microscope) is shown in Figure 82 to Figure 84 . The chips were fabricated by TSMC in China.

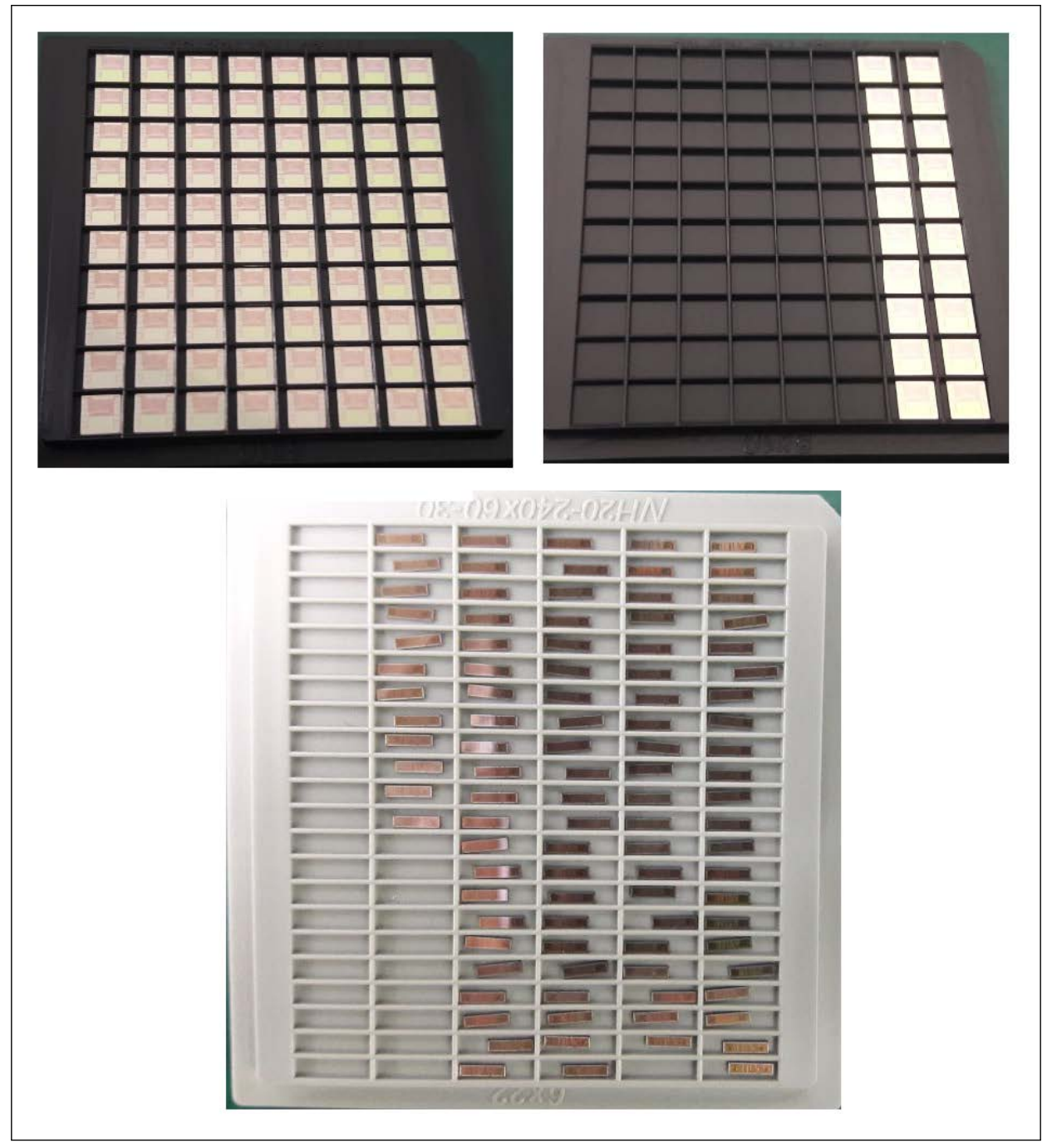

Figure 81: Images of the ADPLL chips containing the DCO that was fabricated. 
A comparison between the layout and the fabricated die of the bottom of the chip is shown in Figure 82.

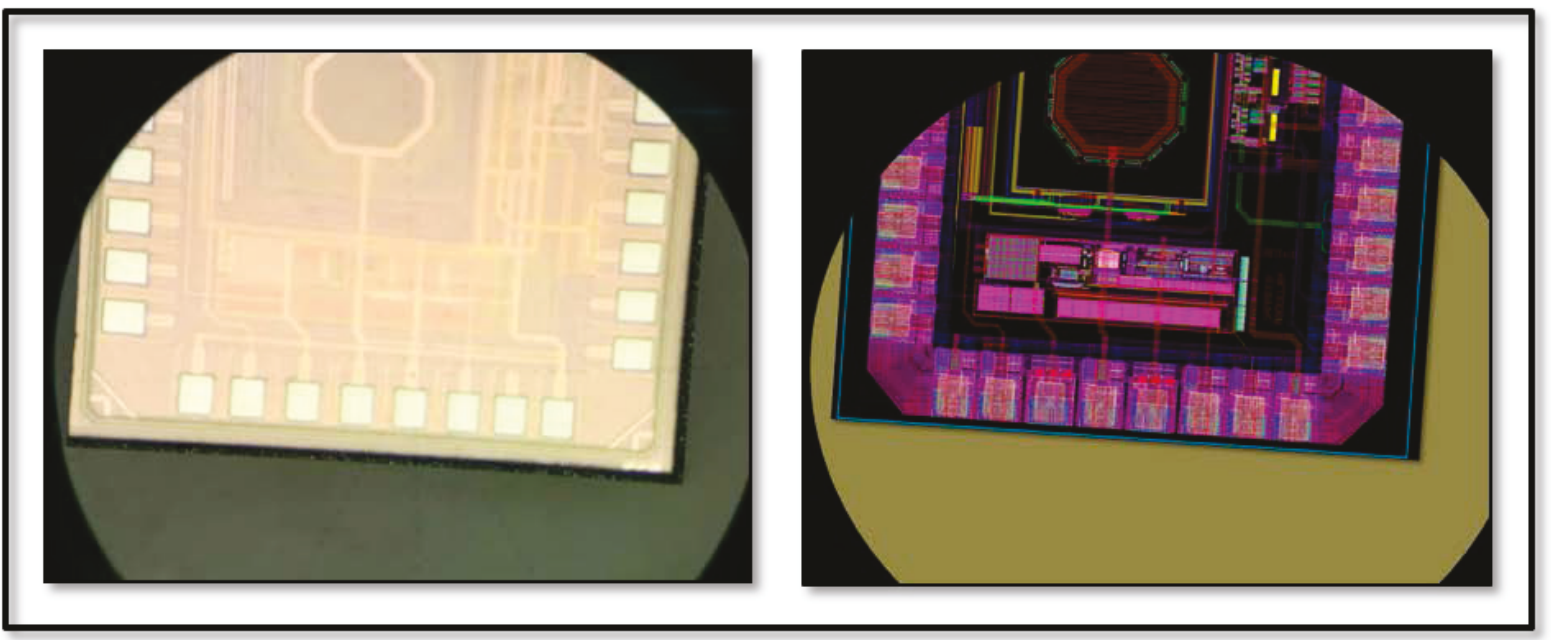

Figure 82: Zoomed in view of the bottom part of the die showing the inductor and regulator of the DCO. The drawn layout of the chip is placed as a reference on the right side.

A comparison of the top part of the die and its layout containing the second DCO is shown in Figure 83.
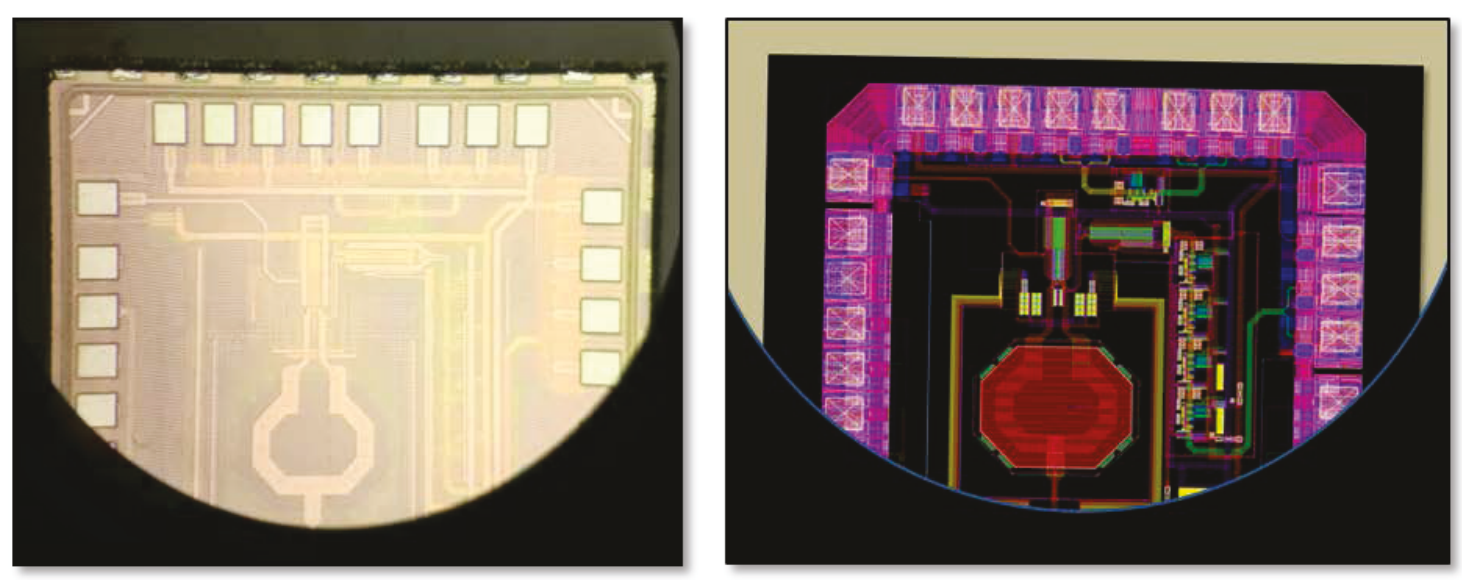

Figure 83: Zoomed in view of the top part of the die showing the layout of DCO 2. The drawn layout of DCO 2 is placed as a reference on the right side.

A comparison of the right side of the chip is shown in Figure 84. 

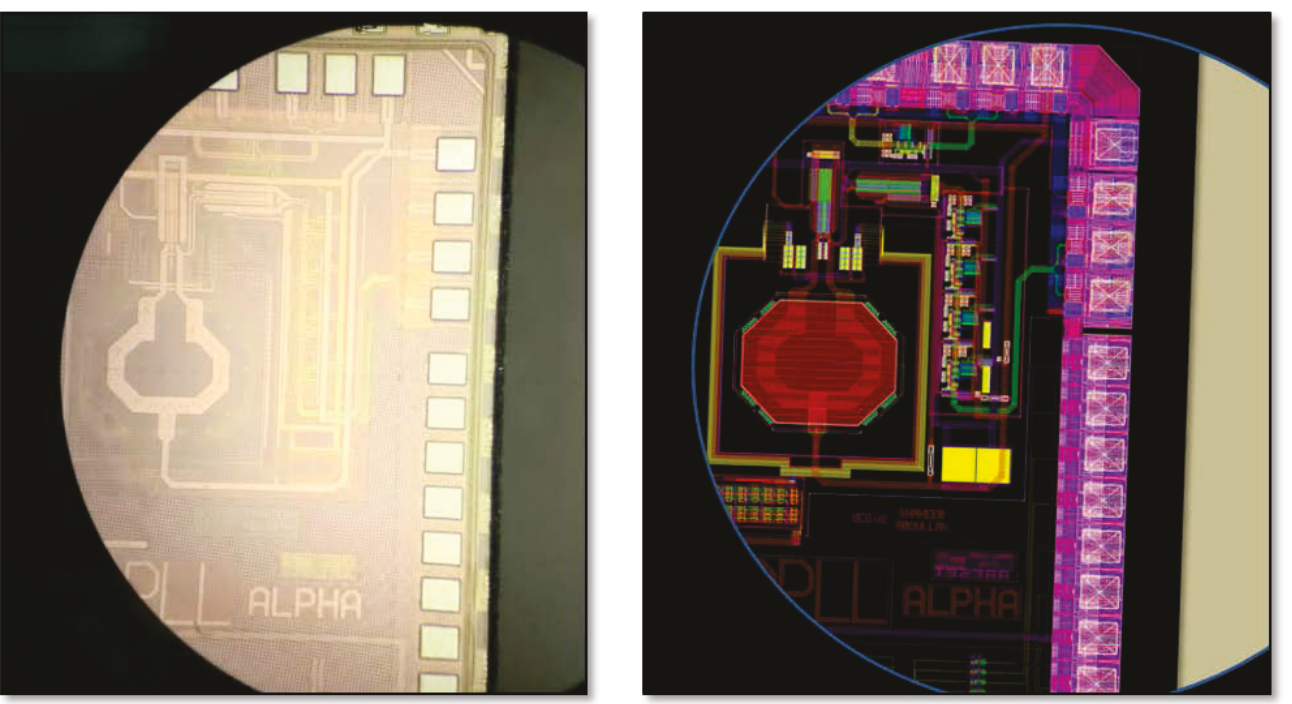

Figure 84: Zoomed in view of the right part of the die. The drawn layout of is placed as a reference on the right image.

\subsection{Bonding and Packaging of Die}

The packaging type selected was QFN64 (Quad-Flat No-leads 64 pin). QFN packaging was chosen since the leads were underneath the plastic body (instead of protruding out like QFP (Quad-Flat Pack) packages). Small leads meant minimized parasitic and hence improved performance; however the drawback of using QFN packaging was that they were difficult to solder and required professional soldering. All 64 pins from the QFN package was required. Images of the final packaged die inside the QFN64 chips is shown in Figure 85 and the die with wire bonding is shown in Figure 86. These chips were then soldered on to test PCBs (Printed Circuit Boards) for testing the DCOs.

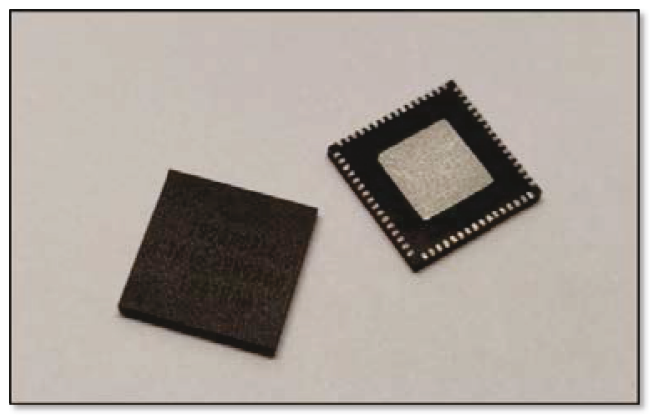

Figure 85: Image of the DCO chips. QFN64 packing was used to house the die. The right chip shows the bottom of the package and the left chip shows the top of the package. 


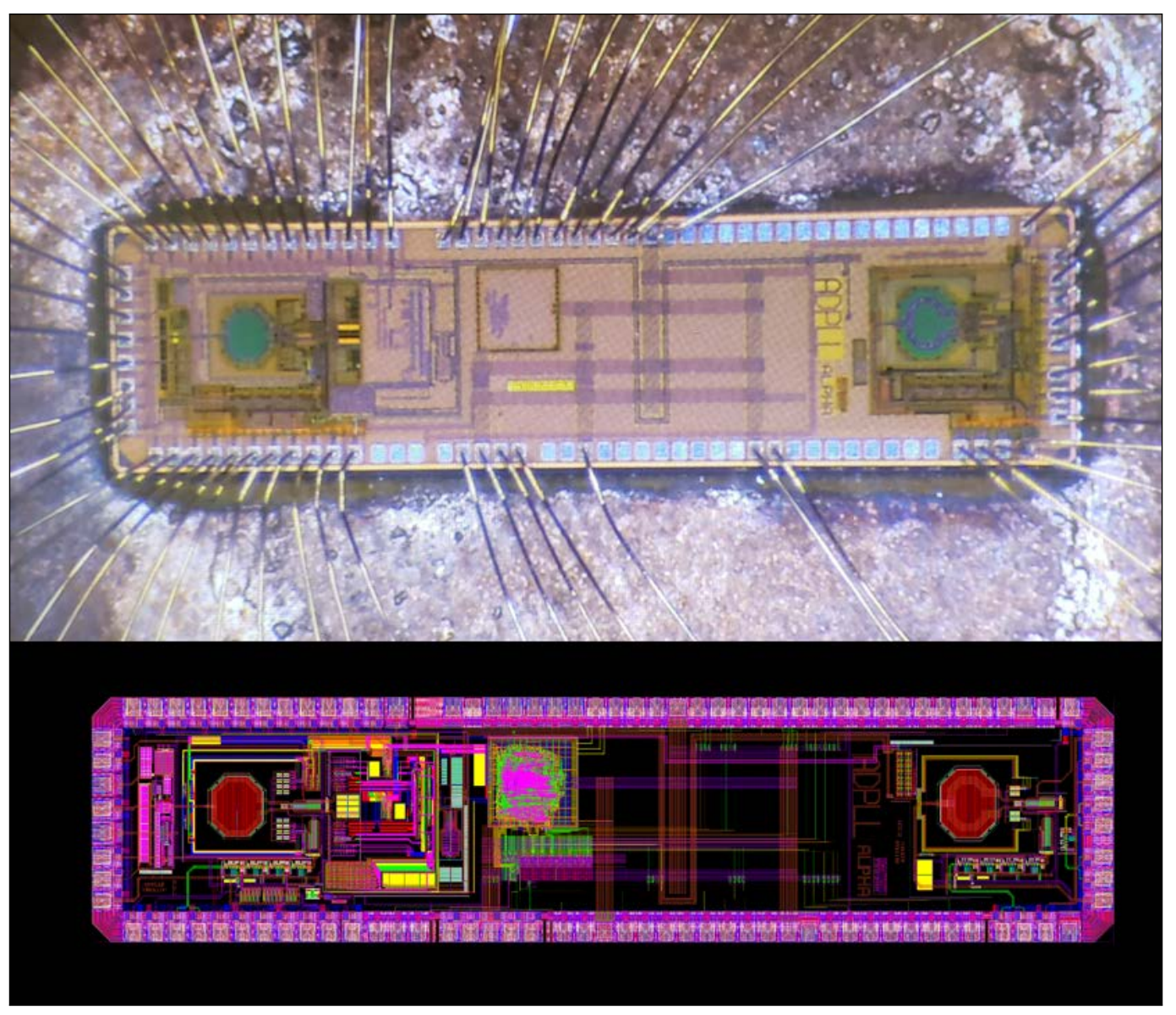

Figure 86: Image of the ADPLL die with bonding wires. Layout shown in bottom for reference.

For bonding diagrams and pin layouts between the packaging and the die see Appendix B.

\subsection{Main Test PCB to Test the DCO}

The design of the main test PCB is shown in Figure 87. See Appendix C for the design of all the layers. RF protocols were kept in check when designing the PCB to ensure maximum efficiency and proper propagation of the signals from the the DCO chip to the SMA connectors for testing. 


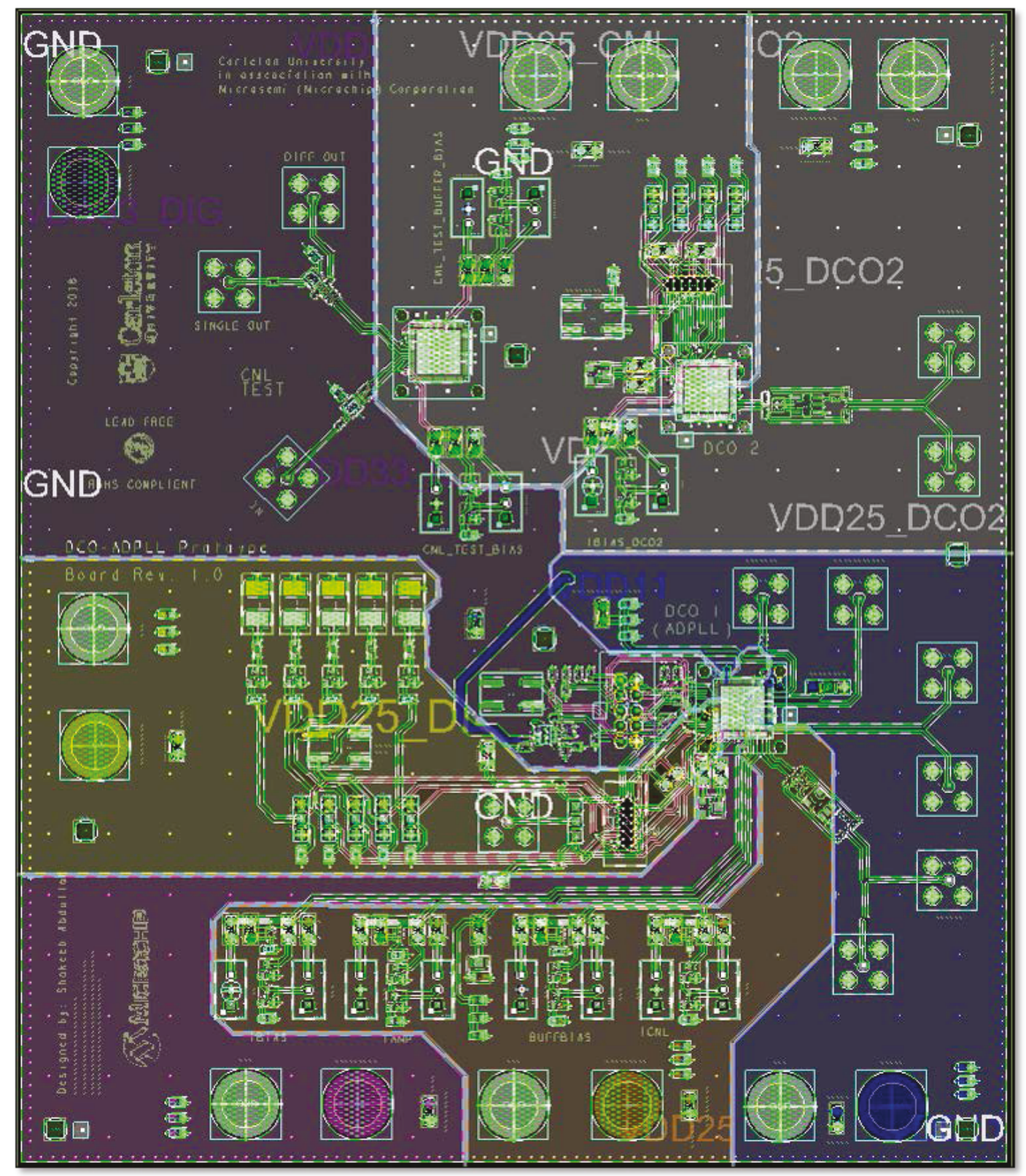

Figure 87: The PCB design of the main test board for the DCO. The PCB was designed by the author of this thesis.

A couple images of the manufactured board with all the components soldered onto it is shown in Figure 88 and 89. The PCB was a four layer PCB. The PCB had three chips soldered on to it and three test set-up (labelled on Figure 88). 


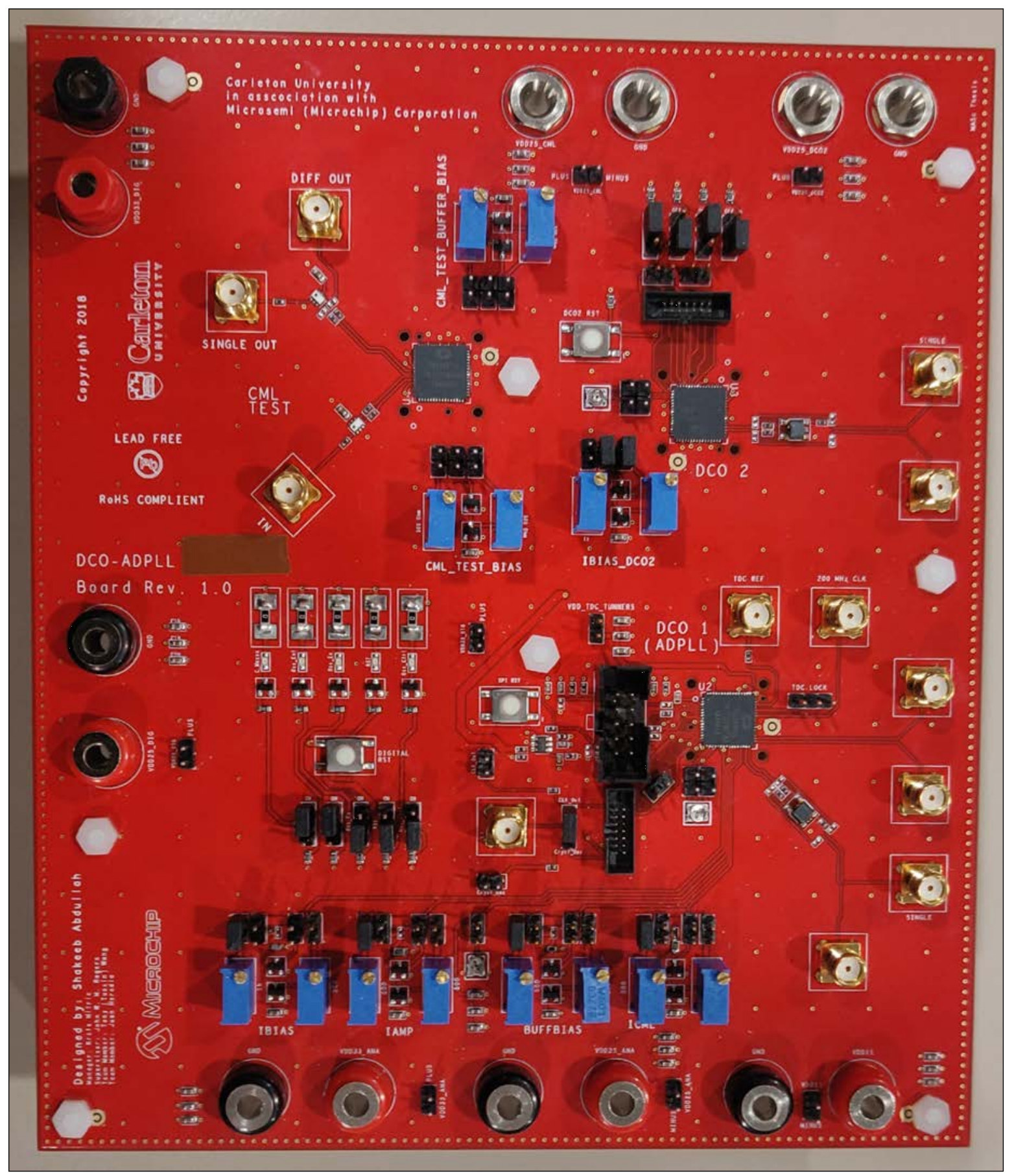

Figure 88: Main test PCB with all the components soldered on (top view). 


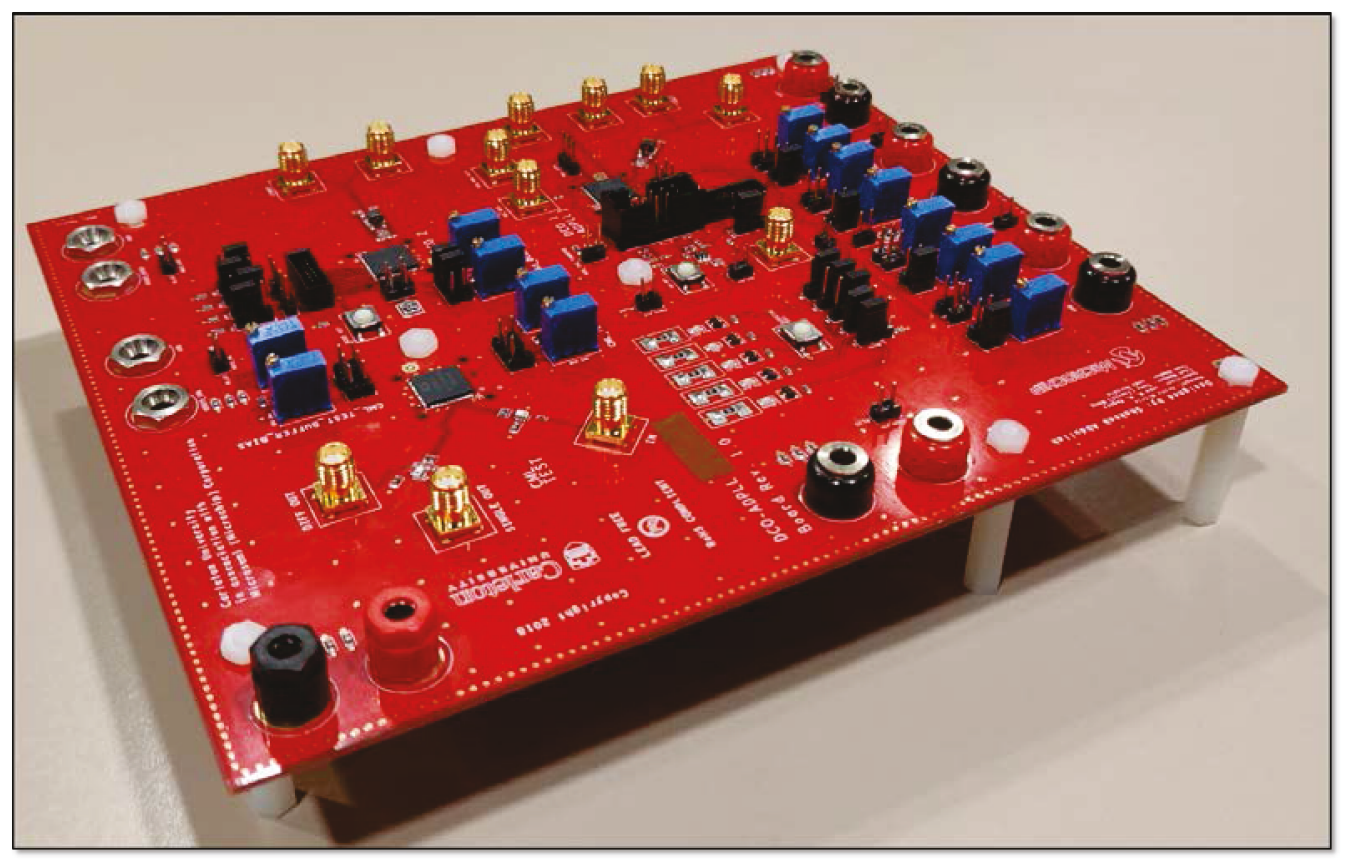

Figure 89: The main test PCB with all components soldered onto it (side view).

\subsection{Digital Test Circuit PCB}

To control the digital circuits of the DCO, a digital PCB interface was created that would interact either with a micro-controller (or an equivalent digital interface such as a parallel port), which in turn would interface with a PC (personal computer) on one end; and on the other end would peripherally talk to the DCO using serial communication. See Figure 90 for a diagram of the communication scheme.

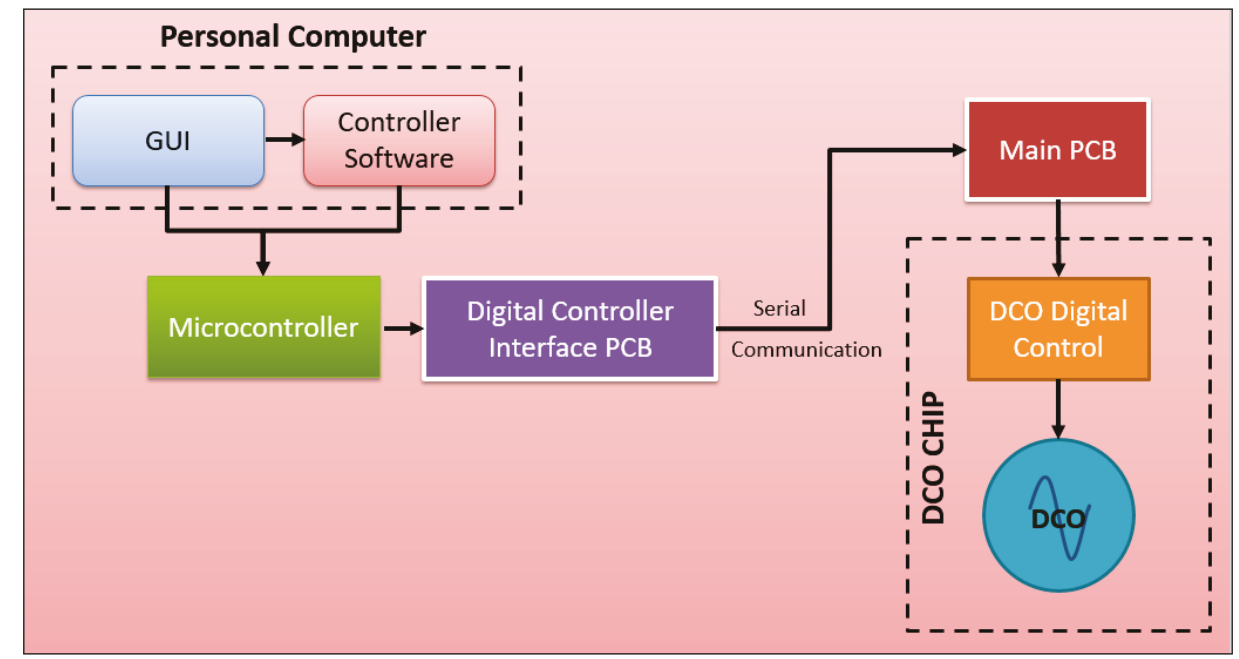

Figure 90: The digital testing protocol/interface of the DCO. 


\subsubsection{Serial communication}

The serial communication was initially implemented using a modified version of the schematic scheme shown in Figure 91. It is a simple parallel to serial converter. A 4-bit test PCB was created to assess if the circuit would work (shown in Figure 92) before creating the 57-bit PCB. See Appendix C for the PCB design.

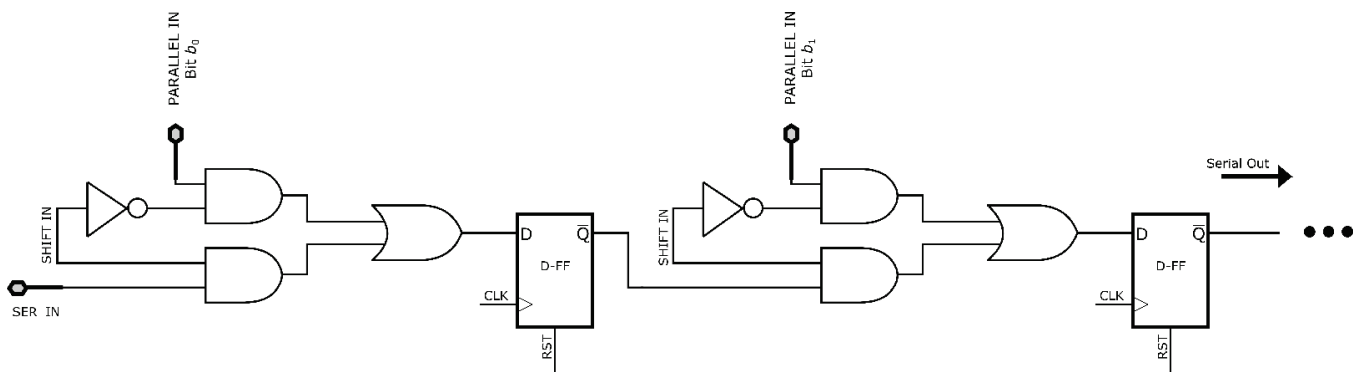

Figure 91: The digital testing protocol of the DCO.

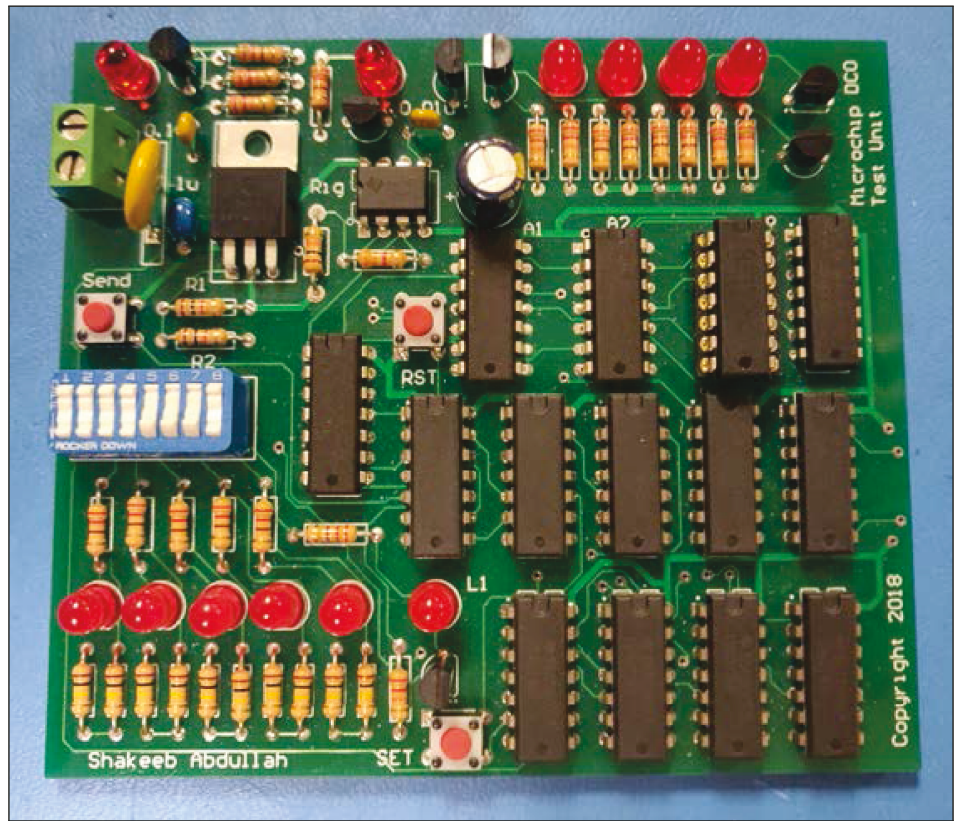

Figure 92: A 4-bit DCO test PCB digital interface. PCB designed by the author of this thesis.

The above PCB (in Figure 92) worked as intended; however designing 57-bit parallel-to-serial interface employing the circuit scheme in Figure 91 and using only 'off the shelf' components/parts was neither practical nor feasible. To resolve the issue, the functioning of an 8-bit parallel-to-serial IC 4014 was modified and daisy chained. 


\subsubsection{PCB of DCO digital interface}

The 3D rendering design of the DCO 57-bit digital-controller-interface (DCO-DCI) is shown in Figure 93 and the fabricated physical PCB with all components soldered on to it is shown in Figure 94. The DCI PCB was also designed to control other functions of the DCO but will not be discussed in this thesis. The DCI PCB board was the main digital test PCB that would interface with the main DCO PCB (from Figure 89).

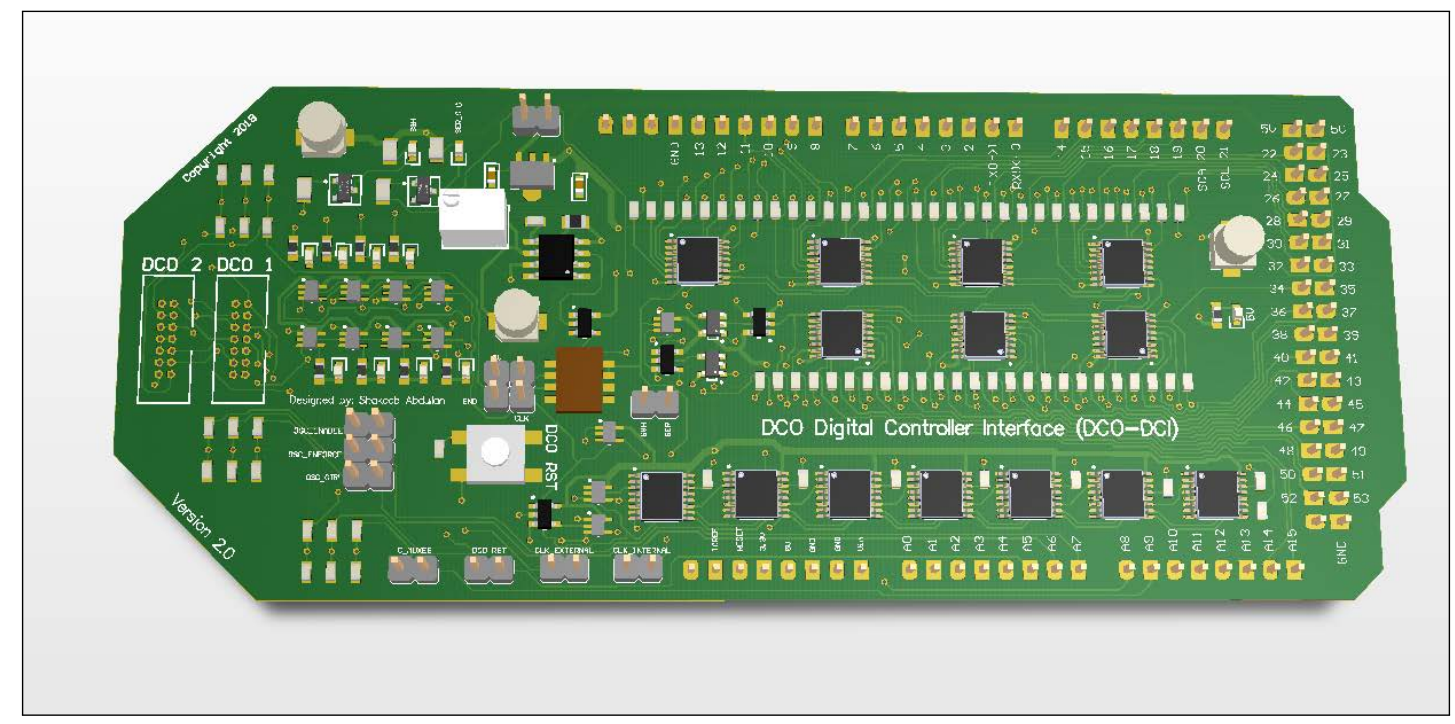

Figure 93: The 3D rendering desing of the DCI PCB.

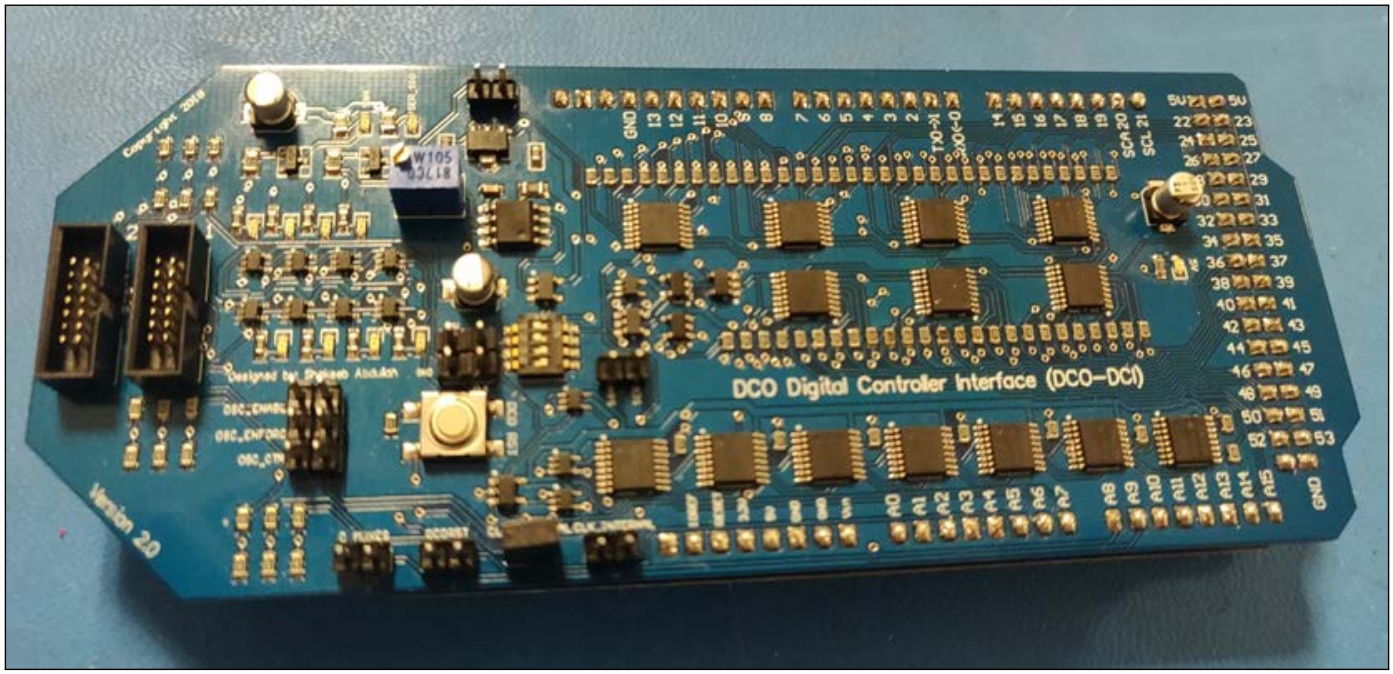

Figure 94: The DCI-DCO controller PCB with all components soldered. PCB designed by the author of this thesis.

A second version of the PCB for the second DCO was also created shown in Figure 95. 


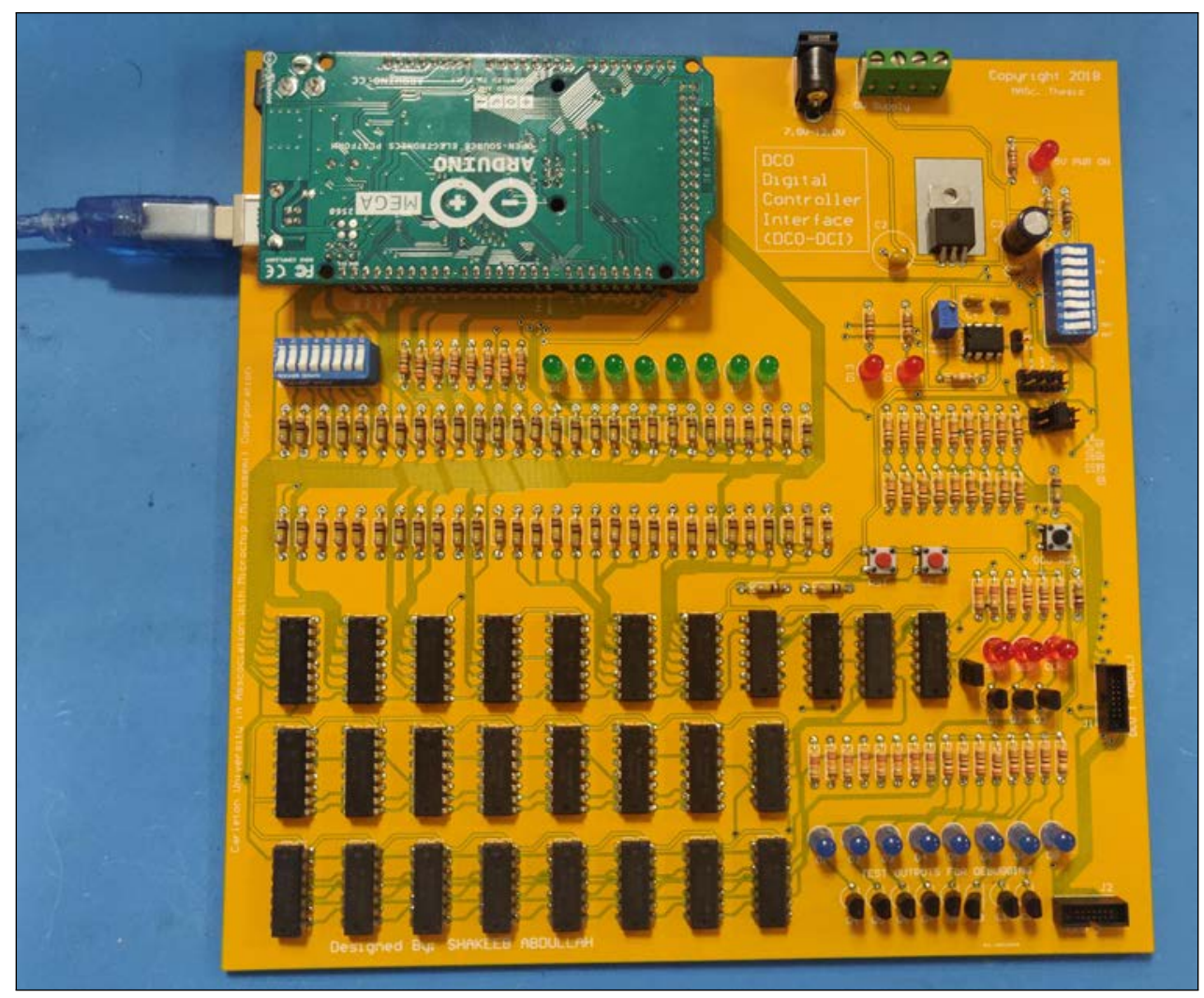

Figure 95: Slightly modified (version 2) DCI PCB with all components soldered. PCB designed by the author of this thesis.

Creating the digital test PCB was almost as challenging as designing the DCO itself. It was of utmost and of extreme cruciality that the digital control PCB worked because without it functioning properly the DCO itself would be useless; nothing of the DCO could be controlled or manipulated and therefore the assessment of the DCO could not take place. Providentially, the digital PCBs worked in its first design cycle.

\subsection{Software to Automate Testing and Control the DCO}

A software with a Graphical User Interface (GUI) using Microsoft (MS) Visual Studio (containing $\mathrm{C \#}$ ) was created to simplify the testing procedure. The software was designed by the author. Testing the DCO one frequency/bit at a time is time consuming and labour intensive. The software was designed to not only control the various functions of the DCO but to also automate the testing. The DCO has 9 banks with 16 bits, 1 bank with 20 bits, and 1 bank with 64 bits. To step through every possible iteration/permutation $(P)$ with would require: 


$$
P=\sum_{\text {bank }_{\text {set }_{1}}}^{\text {bank }_{\text {set }_{n}}}=n_{\#_{\text {of } f_{b} \text { anks }}}=16^{9} \cdot 20 \cdot 64 \simeq 8.7961 \times 10^{13} \text { iterations }
$$

If each iterations took 1 second, then it would require almost 70 million years to go through them all, of course not all iterations are necessary and the confidence of the information can be interpreted from a subset of measurements; even then 3 banks polled together would require 4069 iterations. The software was designed to automate any 3 combinations of banks at a time. See Figure 96 for the GUI of the software.

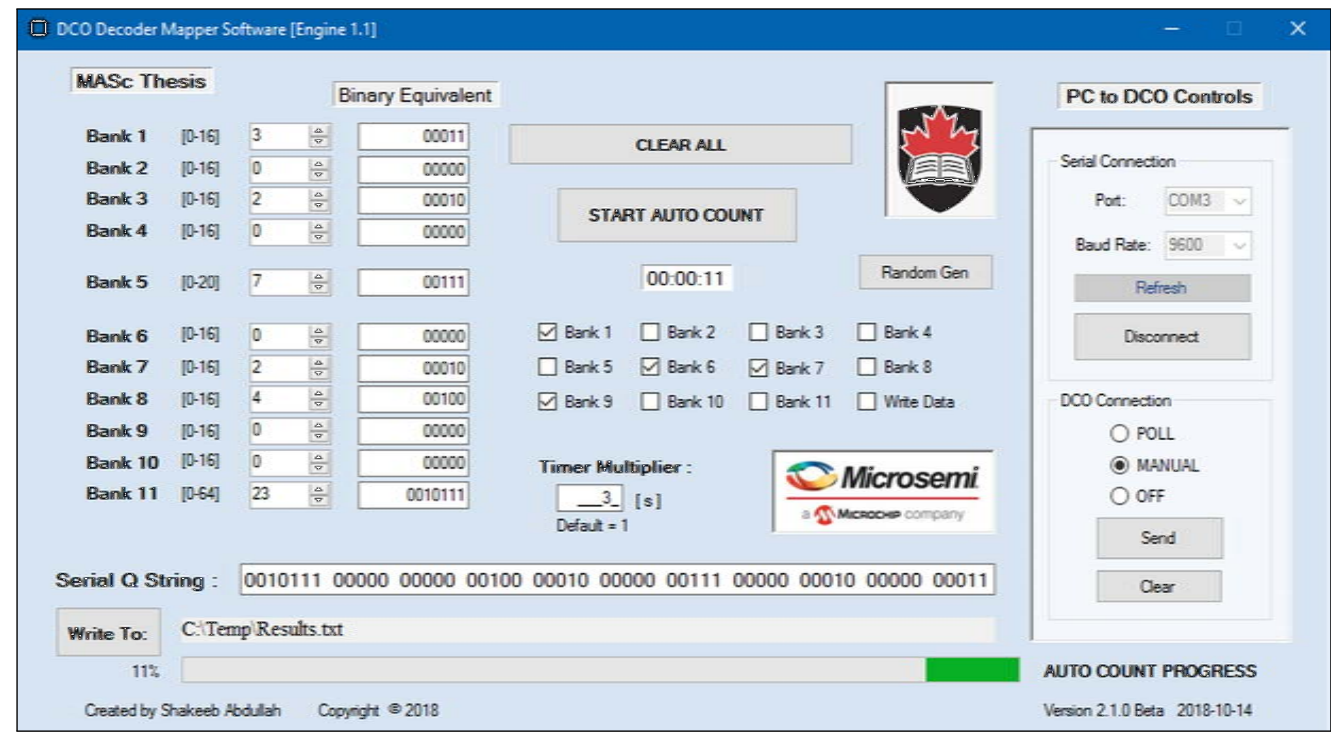

Figure 96: Graphical User Interface (GUI) of the software created to auto test the DCO. GUI software programmed and designed by the author of this thesis.

See Appendix D for an explanation of the various software controls.

\subsection{Additional Test Circuit PCB}

Some of the additional test circuit PCB that was built and used to debug the DCO is shown in Figure 97. 

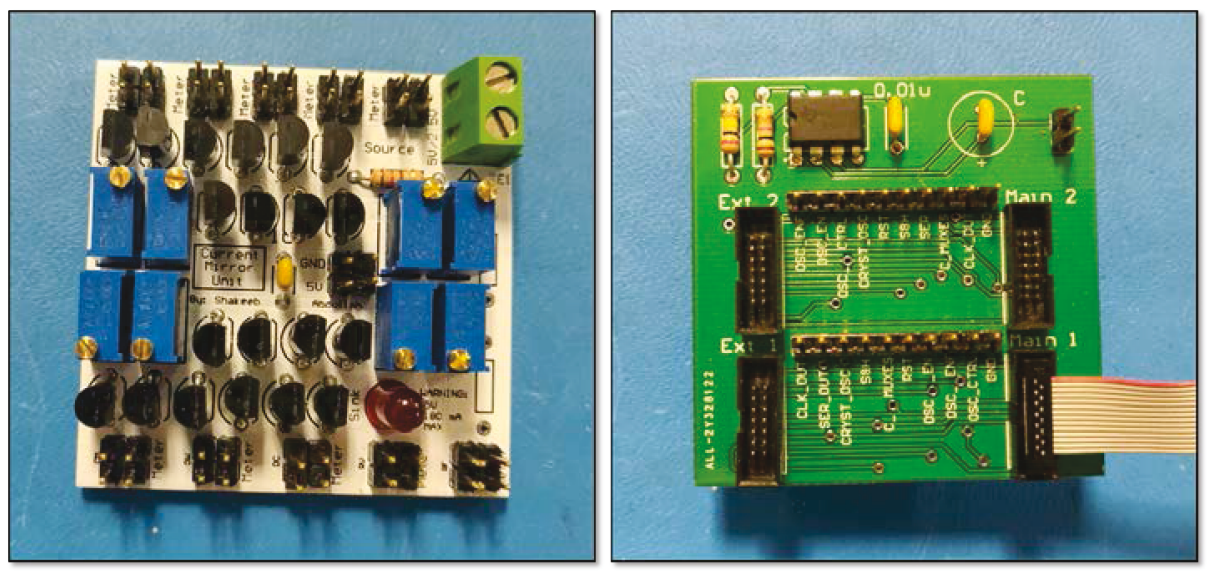

Figure 97: Test PCBs for debugging the DCO. PCBs designed by the author of this thesis.

The right PCB was created for debugging the connections between the DCI PCB and the DCO, and the left PCB was made to debug the bias connections of the DCO.

\subsection{Equipment Used to Test DCO}

The equipment used to test the DCO and peripheral circuits were a spectrum analyzer, signal generator, frequency meters, oscilloscope, phase noise machine, power supplies, and multi-meters. A sample lab test set-up is shown in Figure 98 and a zoomed in view of the PCB test board set-up is shown in Figure 99. 


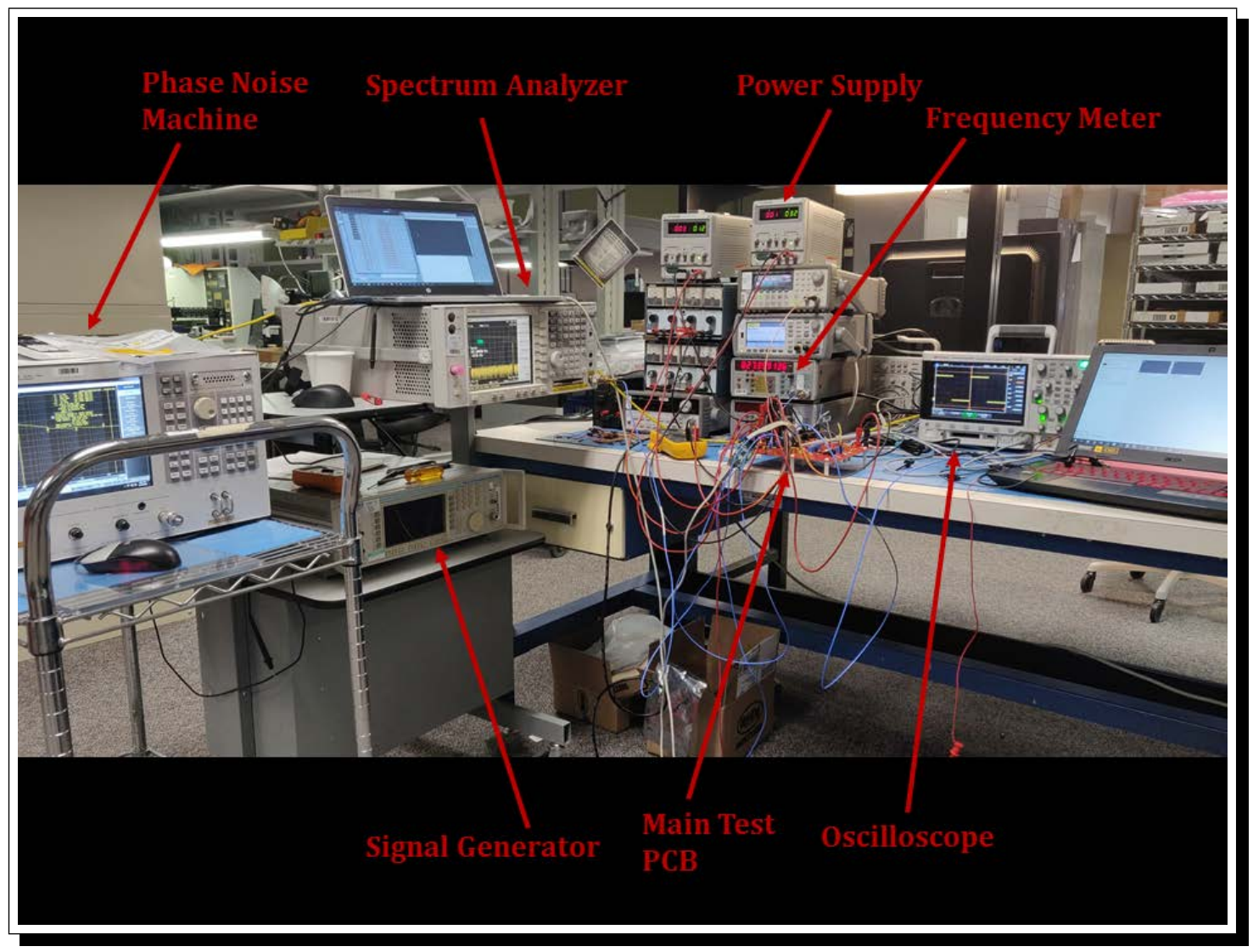

Figure 98: Equipment test setup for evaluating the fabricated DCO. 


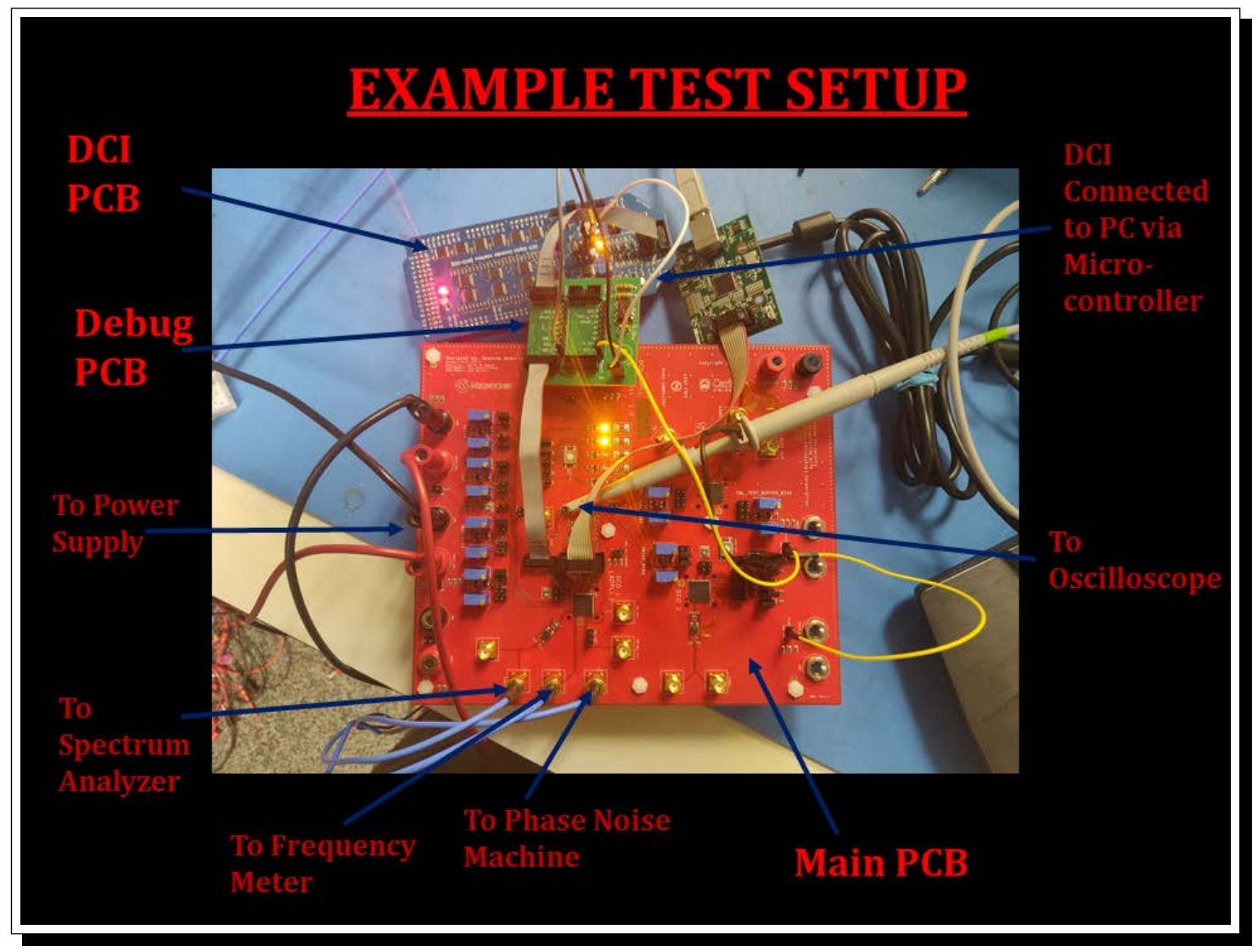

Figure 99: PCB test setup for measuring the performance of the DCO.

The results and measurements obtained from the test equipment will be presented in the next Chapter.

\subsection{Conclusion}

This chapter looked at the test setup used to measure the performance of the fabricated DCO starting with the test chip/die and bonding package. It then looked at the various PCBs used in the testing procedure of the DCO including the main board and the DCO-DCI PCB. The chapter also looked at the software that was used to automate the testing procedure of the DCO. The chapter concluded by looking at the test setup and measuring equipment used to measure the performance of the DCO. 


\section{CHAPTER 9: EXPERIMENTAL RESULTS}

This chapter will discuss the measured results obtained from the DCO. All results in this Chapter were done under room temperature and nominal conditions.

\subsection{Transient Results}

The transient results of the CML outputs will be shown in this sub-chapter.

\subsubsection{Transient results of oscillator}

The transient result of the DCO signal when all banks are off after it has been divided by 16 (4 CML divisions) observed on an oscilloscope is shown in Figure 100.

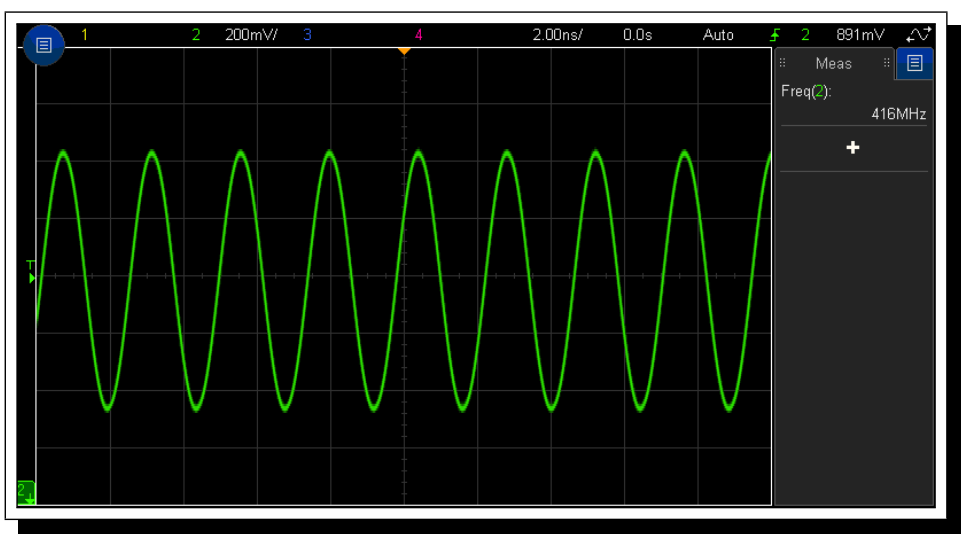

Figure 100: Divide by 16 signal of the DCO measured on an oscilloscope when all the banks are off.

The transient result of the DCO signal when all banks are on, observed on an oscilloscope after it has been divided by 16 (4 CML divisions) is shown in Figure 101. 


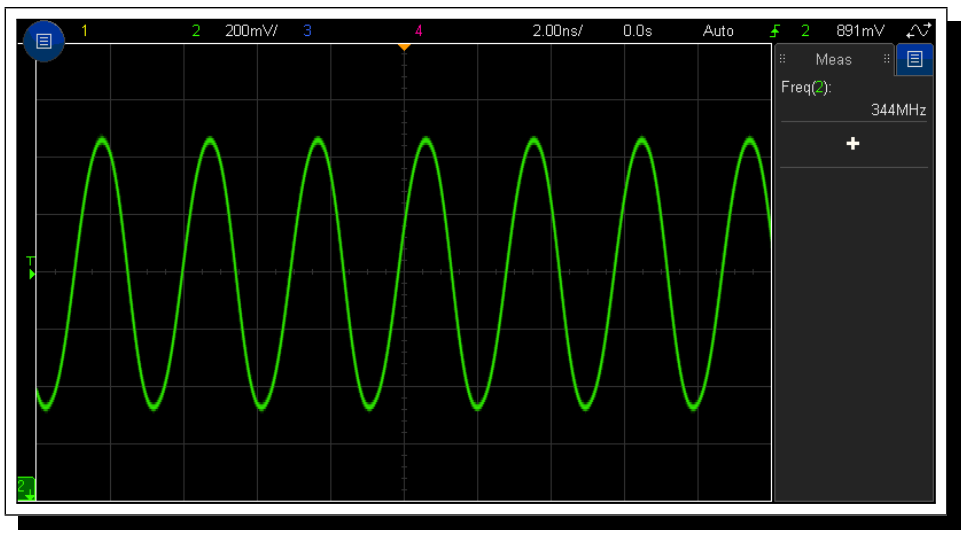

Figure 101: Divide by 16 signal of the DCO measured on an oscilloscope when all the banks are on.

\subsubsection{Transient results of CML divide by 2}

An example measurement of CML division is shown in Figure 102.

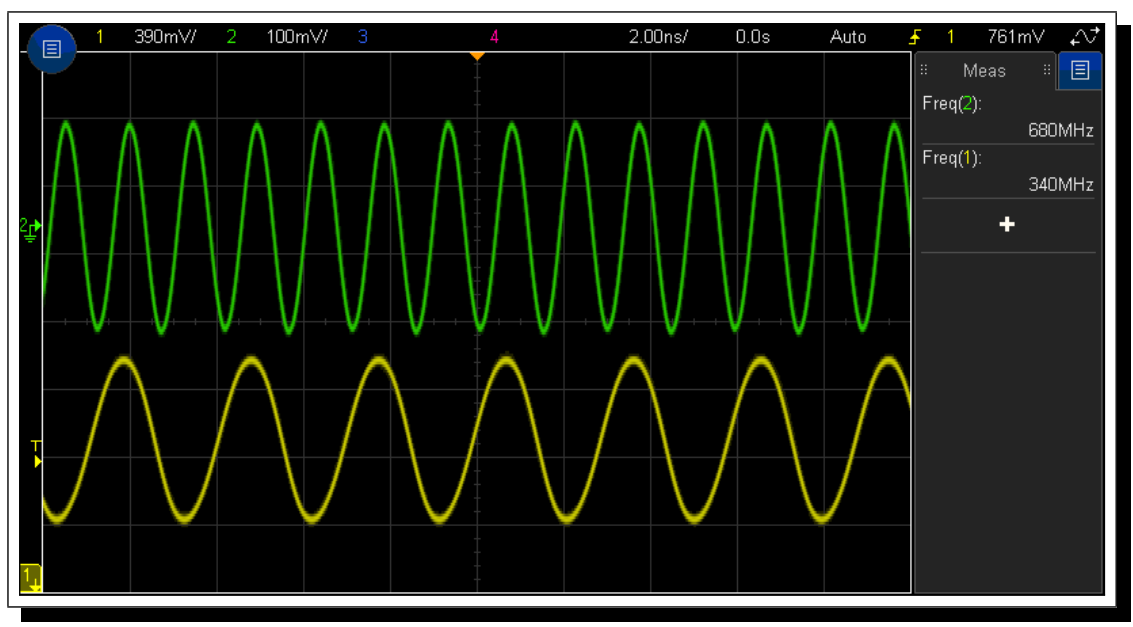

Figure 102: Signals of the CML being divided by 2 .

The bottom signal (yellow) in Figure 102 is an example of a signal that has been divided by 2 .

\subsubsection{Transient simulation of internal ring oscillator (digital clock)}

Measured transient response of the internal (divided down) ring oscillator is shown in Figure 103. 


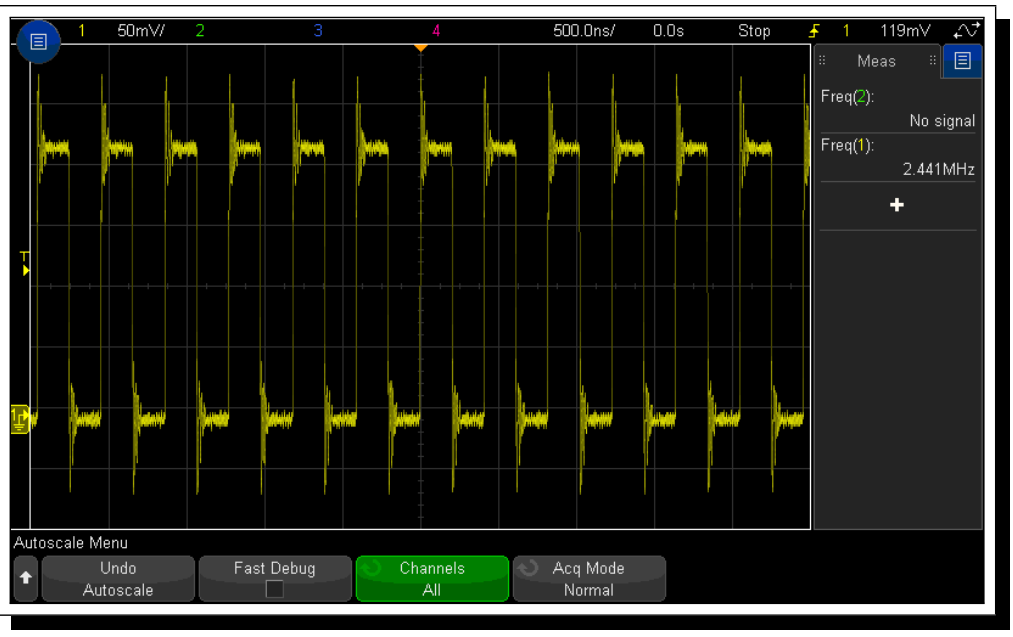

Figure 103: Divided ring oscillator output at maximum frequency.

The variable resistor soldered onto the test PCB to tune the oscillator was not large enough for a wide tuning range however the divided oscillation was still tunable from $2.441 \mathrm{MHz}$ to $1.0915 \mathrm{MHz}$; that means the internal oscillator was tunable from $122.05 \mathrm{MHz}$ to 54.575 $\mathrm{MHz}(\times 50)$. Target output oscillation required for digital clock was $1 \mathrm{MHz}$.

A transient response (divided down) of the oscillator when it is tuned to the lowest frequency is shown in Figure 104.

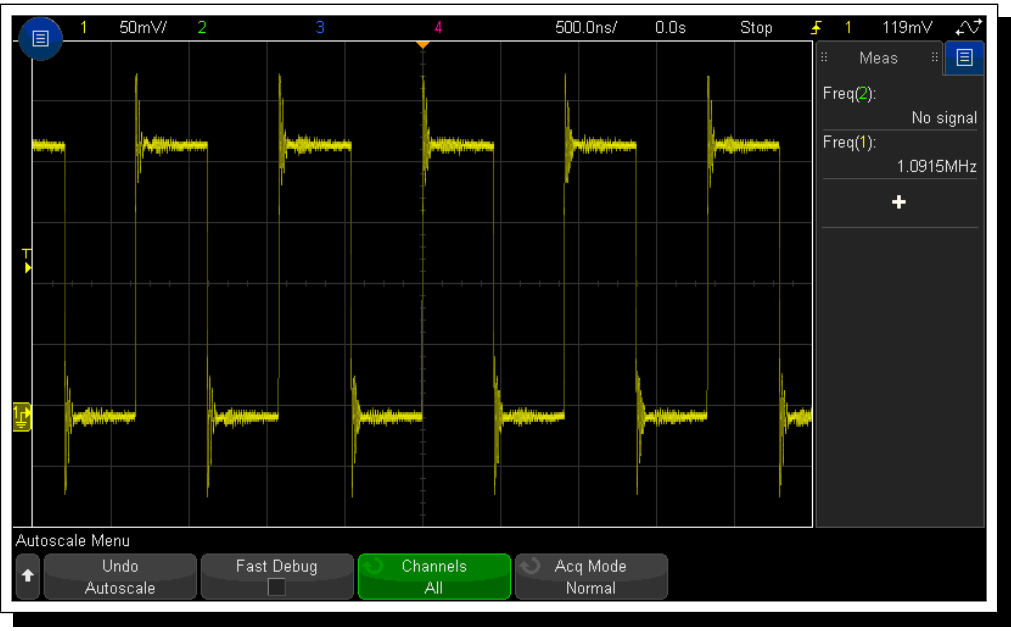

Figure 104: Divided ring oscillator output at minimum frequency.

\subsection{FFT Results}

An example of a FFT (Fast Fourier Transformation) of the oscillator signal divided by 8 when all the banks are off is shown in Figure 105. The measured frequency from the 
analyzer $(\div$ by 8$)$ was $827.0 \mathrm{MHz}$.

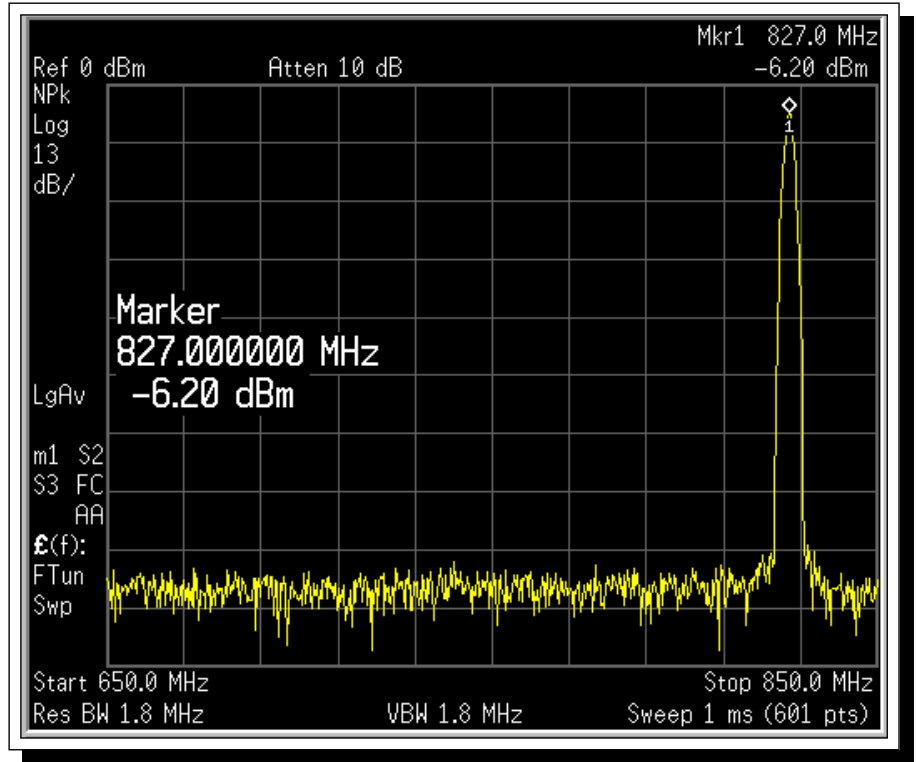

Figure 105: FFT signal (divide by 8) when all banks of the DCO is off. Sweep was $1 \mathrm{~ms}$ and bandwidth was $1.8 \mathrm{MHz}$.

An example of a FFT of the oscillator signal divided by 8 when all banks are on is shown in Figure 106. The measured frequency from the analyzer $(\div$ by 8$)$ was $680.7 \mathrm{MHz}$.

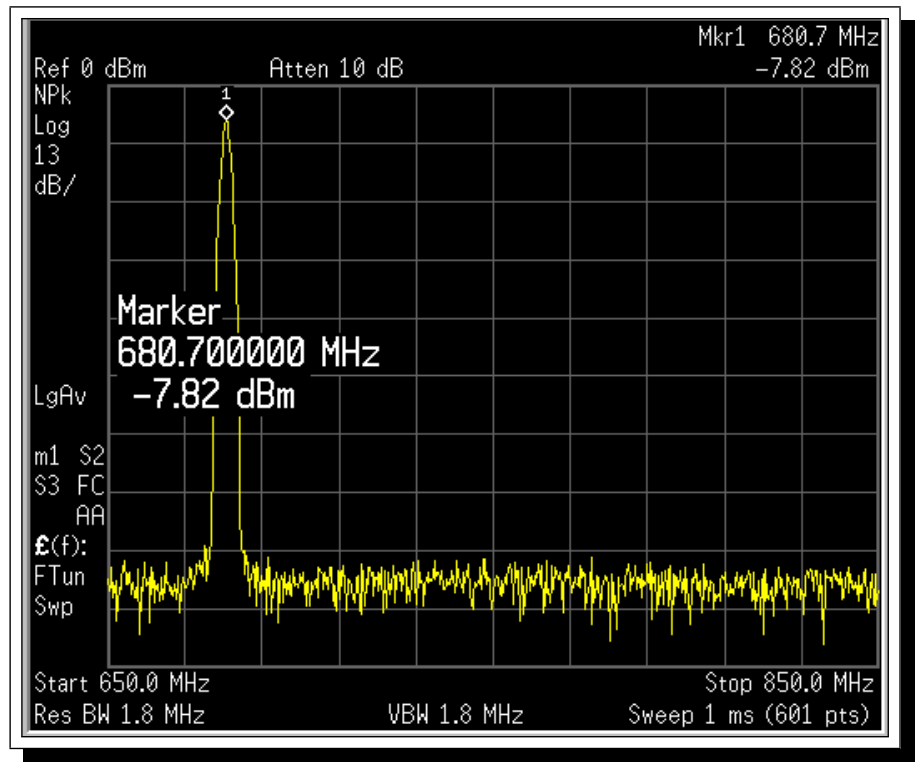

Figure 106: FFT signal (divide by 8) when all banks of the DCO is on. Sweep was $1 \mathrm{~ms}$ and bandwidth was $1.8 \mathrm{MHz}$.

An example of a FFT plot where all the banks of Bank 1 is stepped through and overlaid 
(together) is shown in Figure 107.

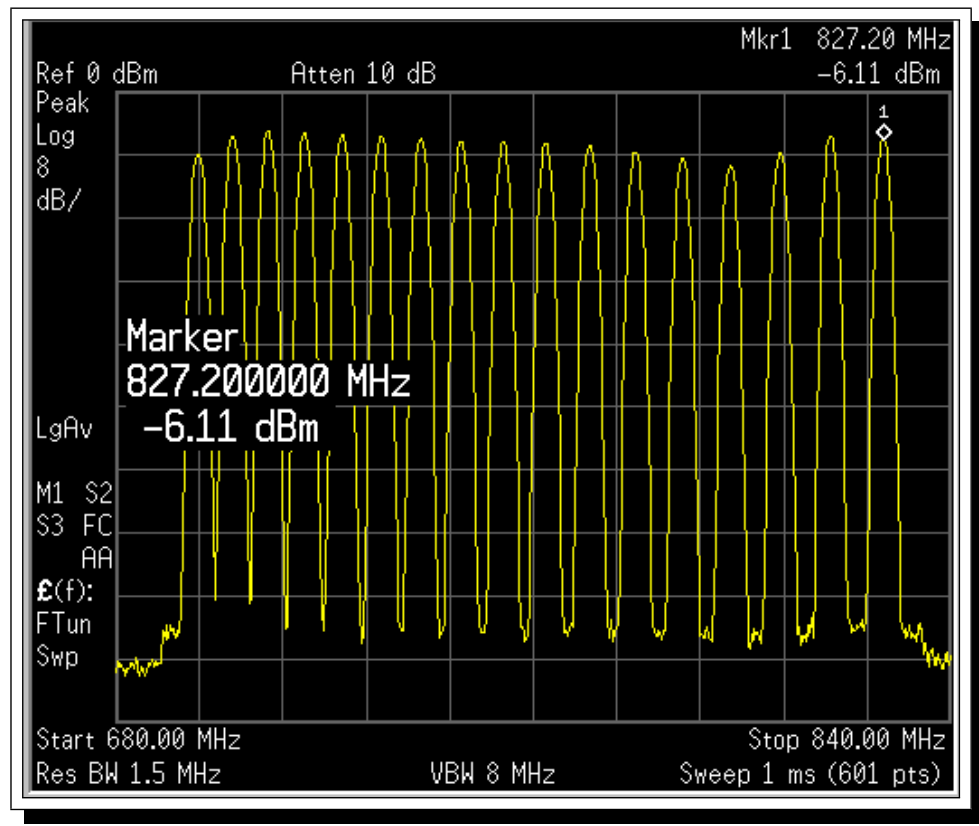

Figure 107: FFT plot of all the bank steps of Bank set 1 overlaid. Sweep was $1 \mathrm{~ms}$ and bandwidth was $1.5 \mathrm{MHz}$.

An example of a FFT plot where all the banks of Bank 2 is stepped through and overlaid (together) is shown in Figure 108.

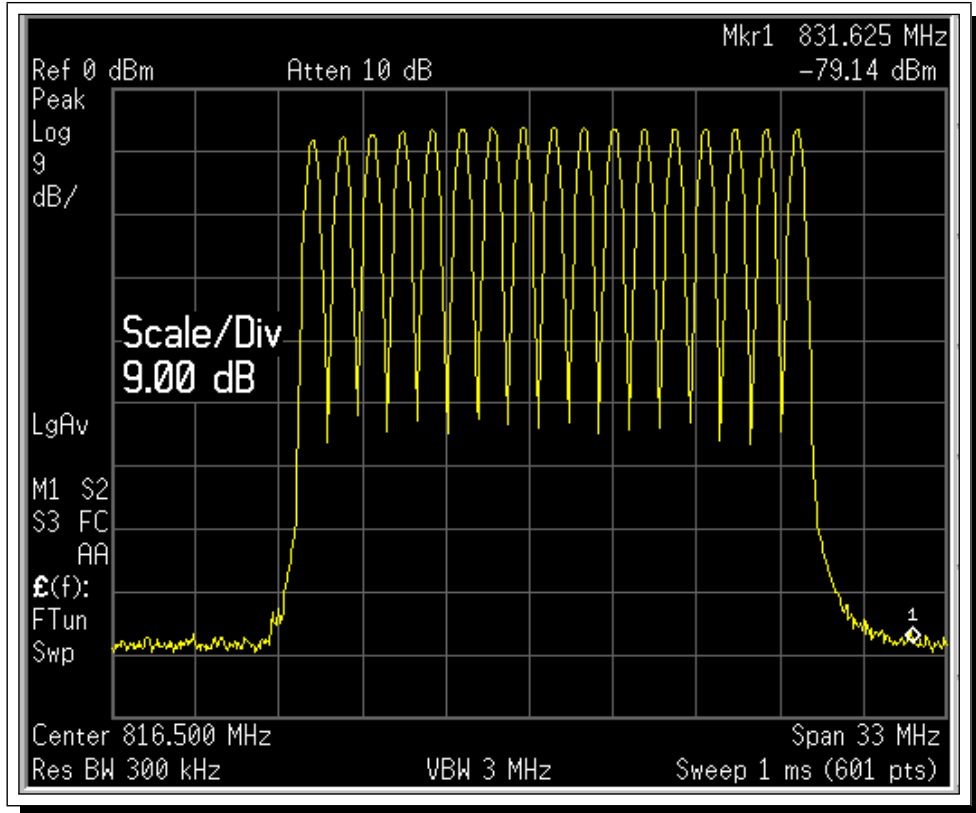

Figure 108: FFT plot of all the bank steps of Bank set 2 overlaid. Sweep was $1 \mathrm{~ms}$ and bandwidth was $300 \mathrm{kHz}$. 
As the frequency steps gets smaller, it gets harder and harder to retrieve a good resolution image from the spectrum analyzer.

\subsection{Frequency Measurements}

The measured frequency response of the DCO will be presented in this section.

\subsubsection{Frequency measurements of Bank set 1}

The measured frequency response of Bank set 1 is shown in Figure 109.

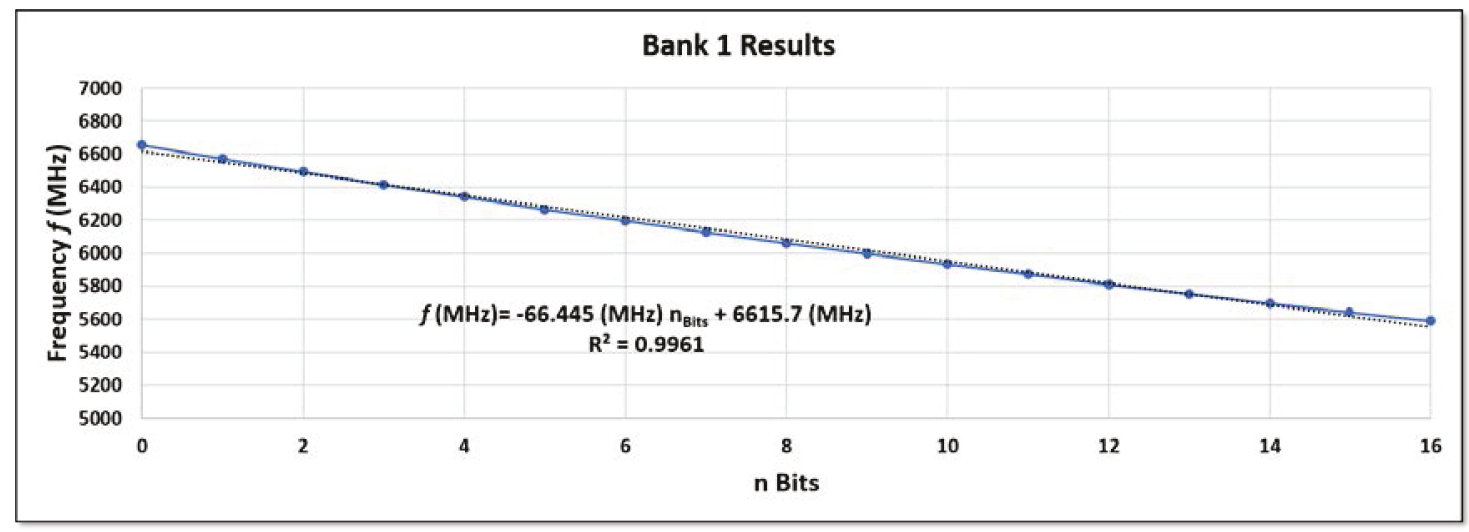

Figure 109: The measured frequency response of Bank 1 steps.

The bank set spans from $6.6560 \mathrm{GHz}$ to $5.5896 \mathrm{GHz}$ and has a linearity of 0.9961 .

\subsubsection{Frequency measurements of Bank set 2}

Since (exhaustively) collecting all data is not feasible, the upper and lower bounds of the bank set was be measured. It was assumed that the frequency response of the banks in between will be bounded by the upper and lower limit frequency responses of the bank set. The measured frequency response of Bank set 2 at the upper limit is shown in Figure 110. 


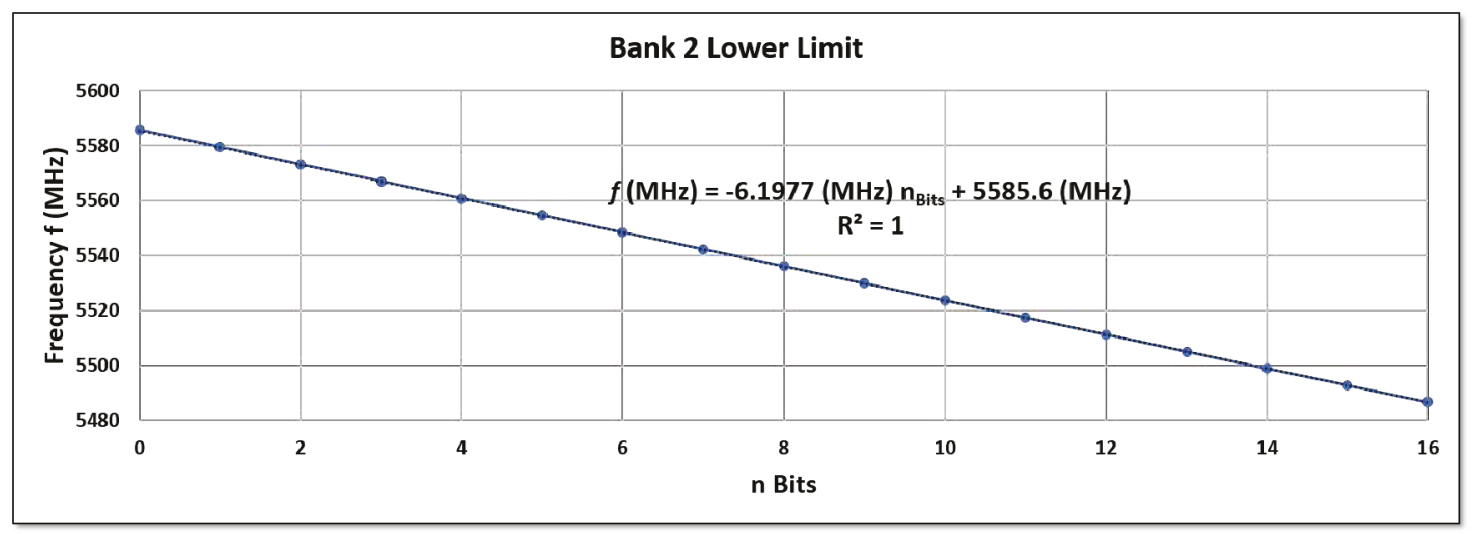

Figure 110: The measured frequency response of Bank set 2 at the upper frequency limit.

The measured frequency response of Bank set 2 at a lower limit is shown in Figure 111.

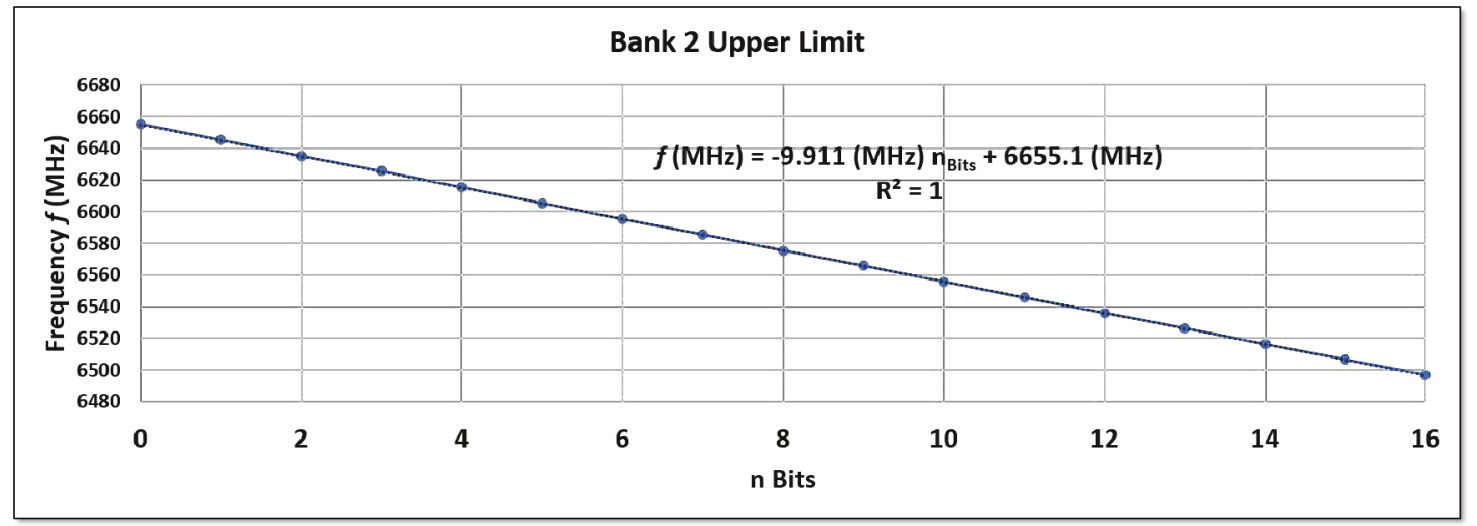

Figure 111: The measured frequency response of Bank set 2 at a lower frequency limit.

\subsubsection{Frequency measurements of Bank set 3}

The measured frequency response of Bank set 3 at the upper limit is shown in Figure 112.

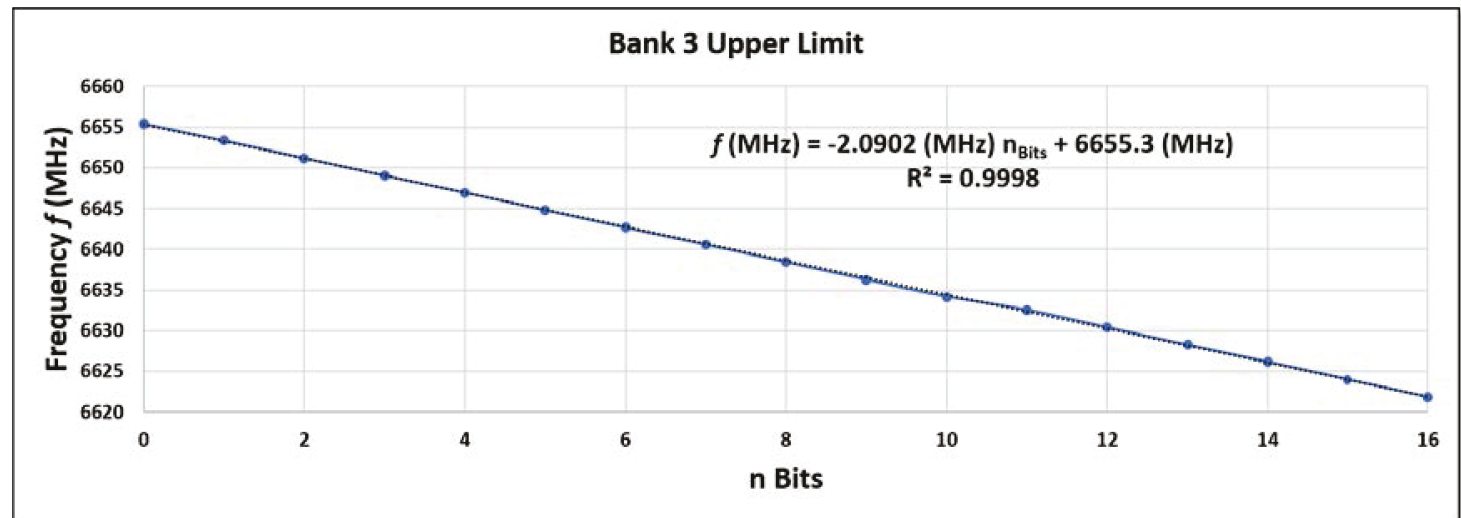

Figure 112: The measured frequency response of Bank set 3 at the upper frequency limit. 
The measured frequency response of Bank set 3 at a lower limit is shown in Figure 113.

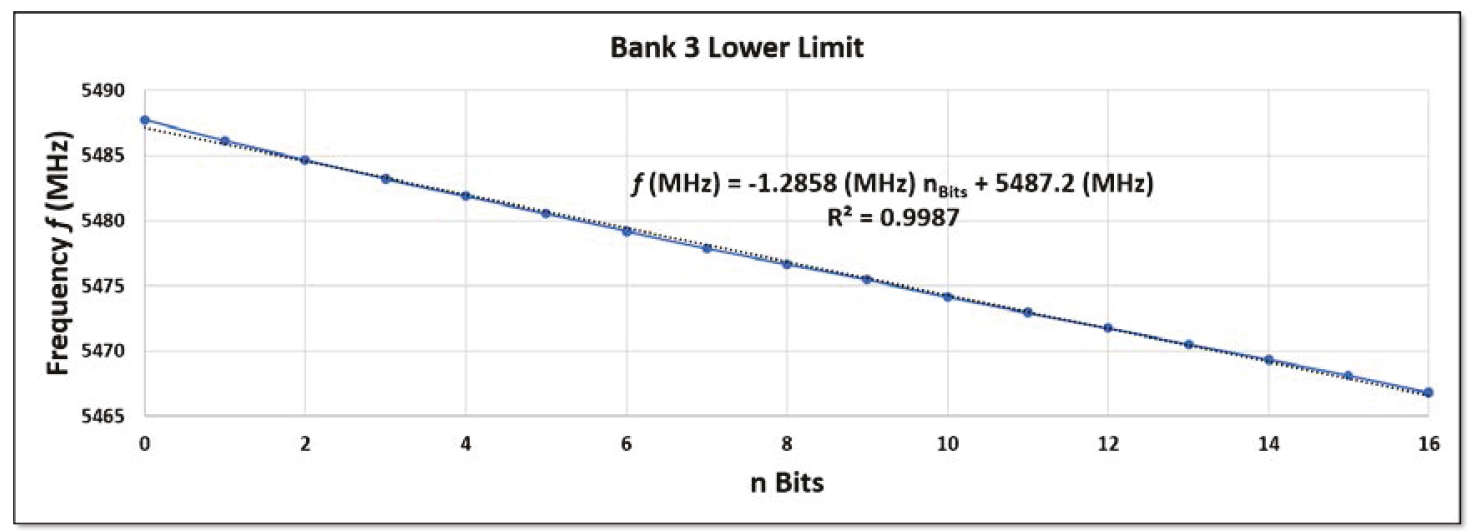

Figure 113: The measured frequency response of Bank set 3 at a lower frequency limit.

\subsubsection{Frequency measurements of Bank set 4}

The measured frequency response of Bank set 4 at the upper limit is shown in Figure 114.

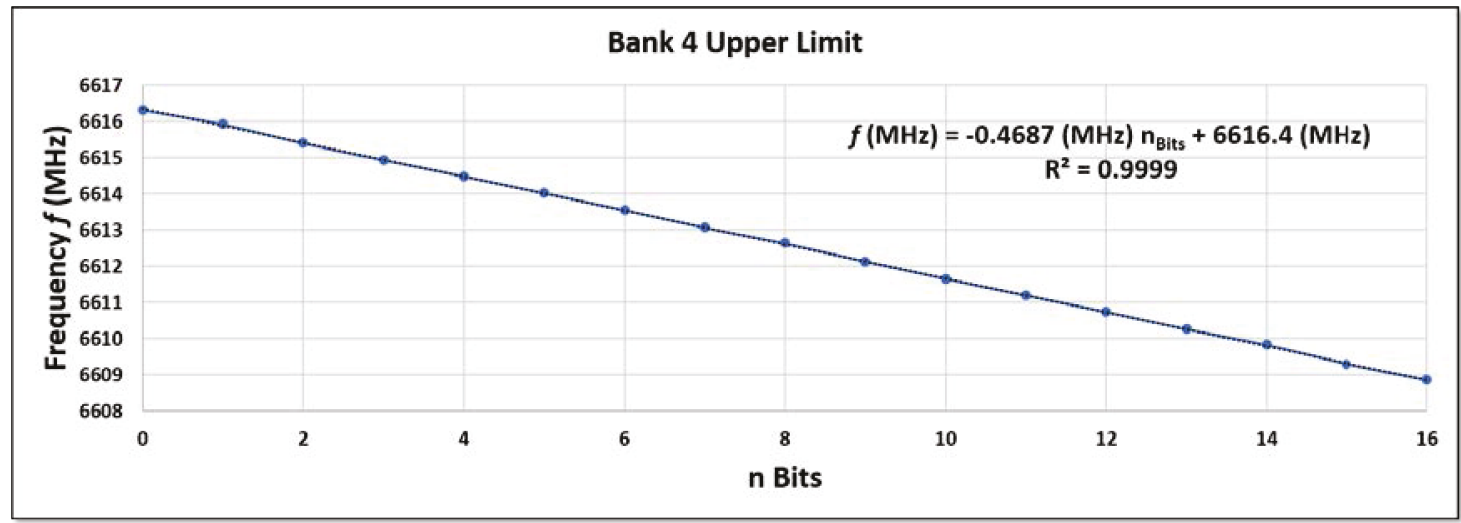

Figure 114: The measured frequency response of Bank set 4 at the upper frequency limit.

The measured frequency response of Bank set 4 at a lower limit is shown in Figure 115. 


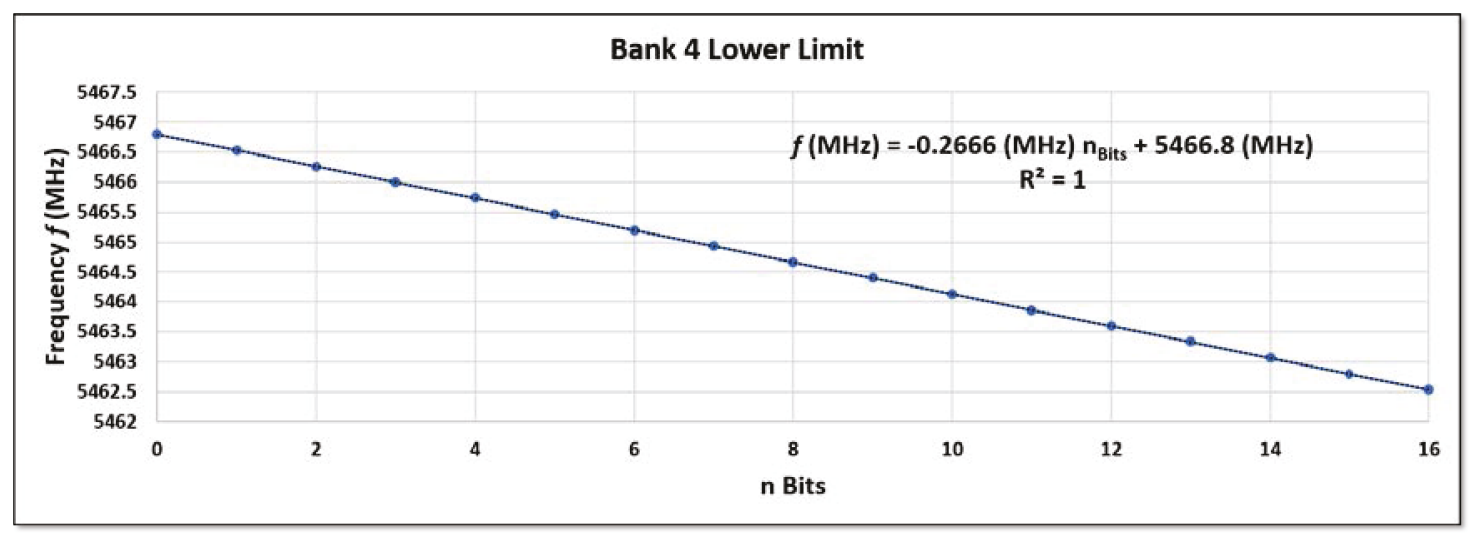

Figure 115: The measured frequency response of Bank set 4 at a lower frequency limit.

\subsubsection{Frequency measurements of Bank set 5}

The measured frequency response of Bank set 5 at the upper limit is shown in Figure 116.

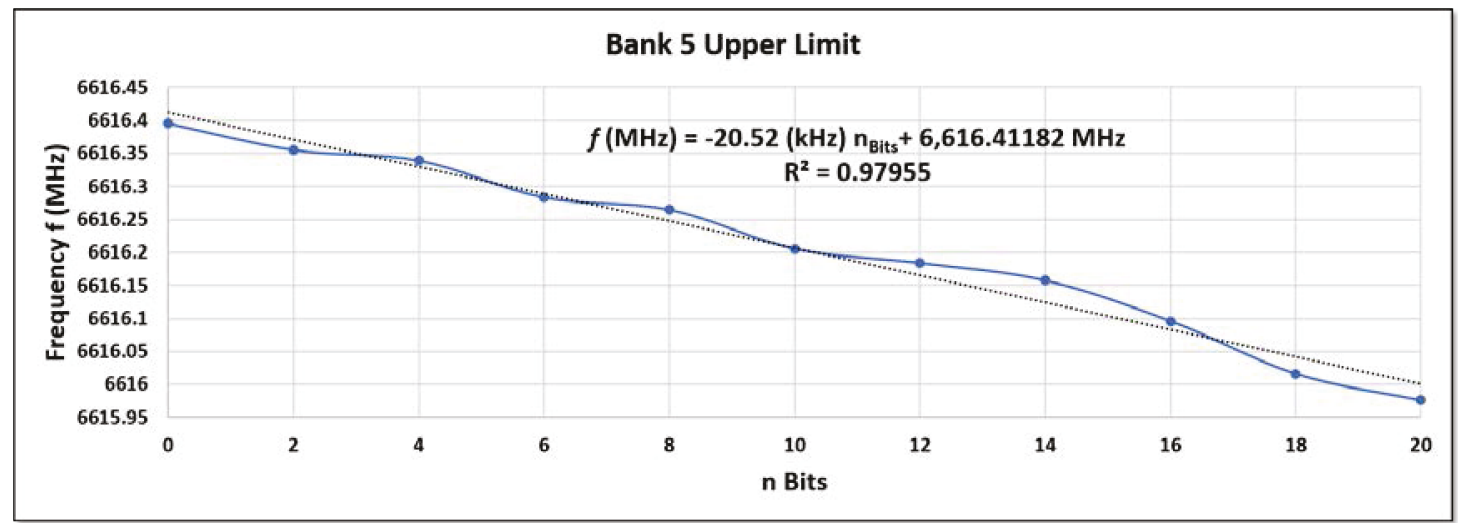

Figure 116: The measured frequency response of Bank set 5 at the upper frequency limit.

The measured frequency response of Bank set 5 at a lower limit is shown in Figure 117.

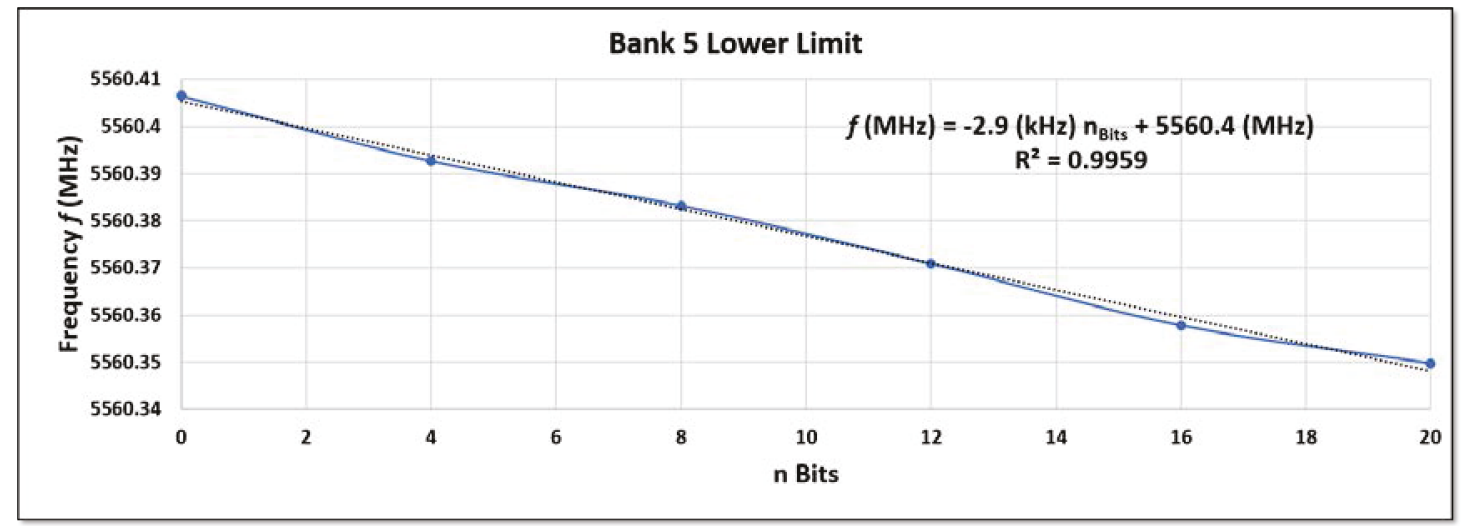

Figure 117: The measured frequency response of Bank set 5 at a lower frequency limit. 


\subsubsection{Frequency measurements of Bank set 6 to Bank set 11}

Unfortunately data for Bank set 6 to Bank set 11 could not be properly obtained (the results were not adequate for presentation in this thesis). However this was expected, since the DCO was purposely designed with smaller and smaller banks to test (and quantify) at what resolution it would fail to respond.

\subsubsection{Summarized Frequency Step Measurements}

Table 4 shows the summarized frequency step measurements for all the bank sets.

TABLE 4: Measured Frequency Step Sizes for the Bank Sets of the DCO.

\begin{tabular}{|c|c|c|}
\hline Bank Number & Number of Bank Units & Measured Frequency Step Size \\
\hline Bank Set 1 & 16 & $66.4 \mathrm{MHz}$ \\
\hline Bank Set 2 Upper & 16 & $9.91 \mathrm{MHz}$ \\
\hline Bank Set 2 Lower & 16 & $6.20 \mathrm{MHz}$ \\
\hline Bank Set 3 Upper & 16 & $2.09 \mathrm{MHz}$ \\
\hline Bank Set 3 Lower & 16 & $1.29 \mathrm{MHz}$ \\
\hline Bank Set 4 Upper & 16 & $469 \mathrm{kHz}$ \\
\hline Bank Set 4 Lower & 16 & $267 \mathrm{kHz}$ \\
\hline Bank Set 5 Upper & 20 & $20.52 \mathrm{kHz}$ \\
\hline Bank Set 5 Lower & 20 & $2.90 \mathrm{kHz}$ \\
\hline Bank Set 6 Upper & 16 & N/A \\
\hline Bank Set 6 Lower & 16 & N/A \\
\hline
\end{tabular}

\subsection{Frequency Settling Time Measurements}

The frequency of the DCO does not switch instantaneously, there is a transition period

from the time the digital signal is applied until the output frequency settles. The banks in Bank set 1 took almost $200 \mathrm{~s}(3 \mathrm{~min} \& 20 \mathrm{sec})$ to settle, see Figure 118. 


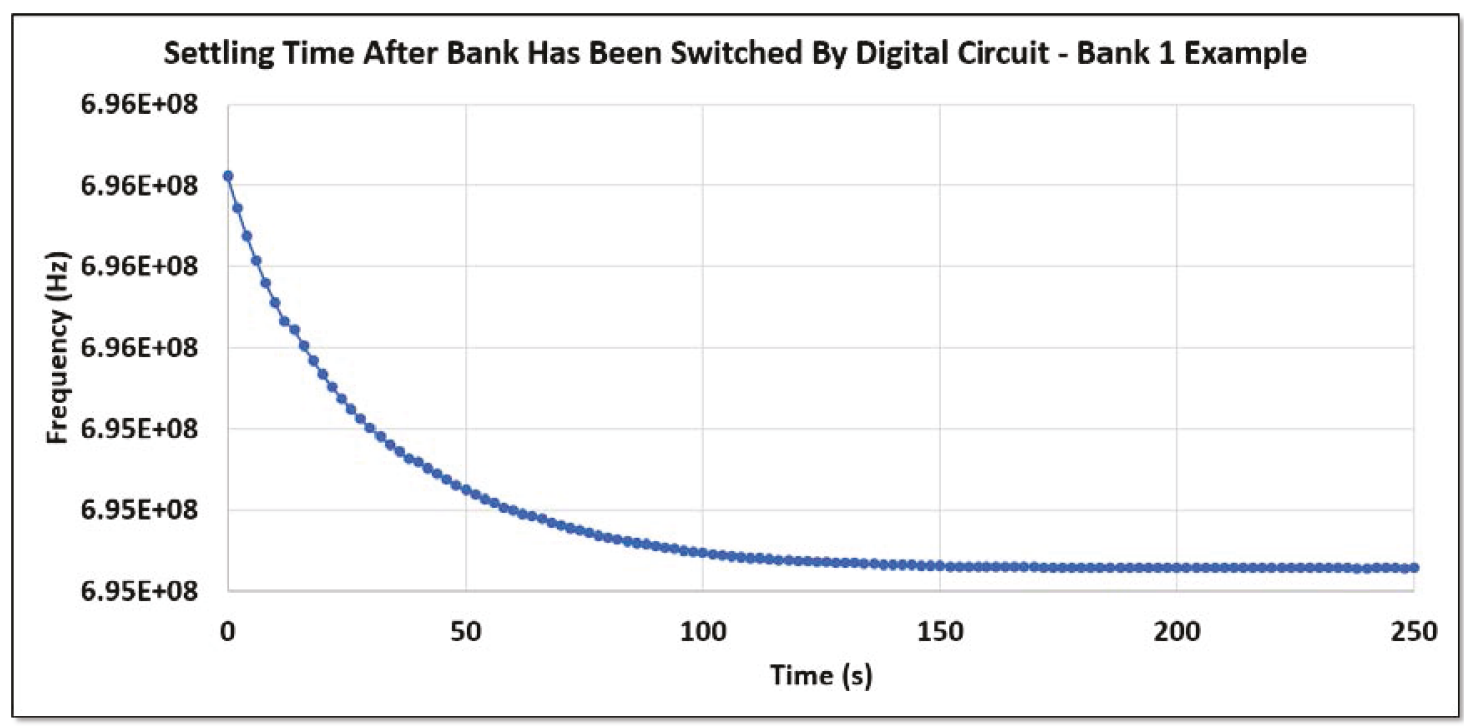

Figure 118: An example of frequency settling time required by banks in Bank Set 1 .

The banks in Bank set 2 took almost $60 \mathrm{~s}(1 \mathrm{~min})$ to settle, see Figure 119.

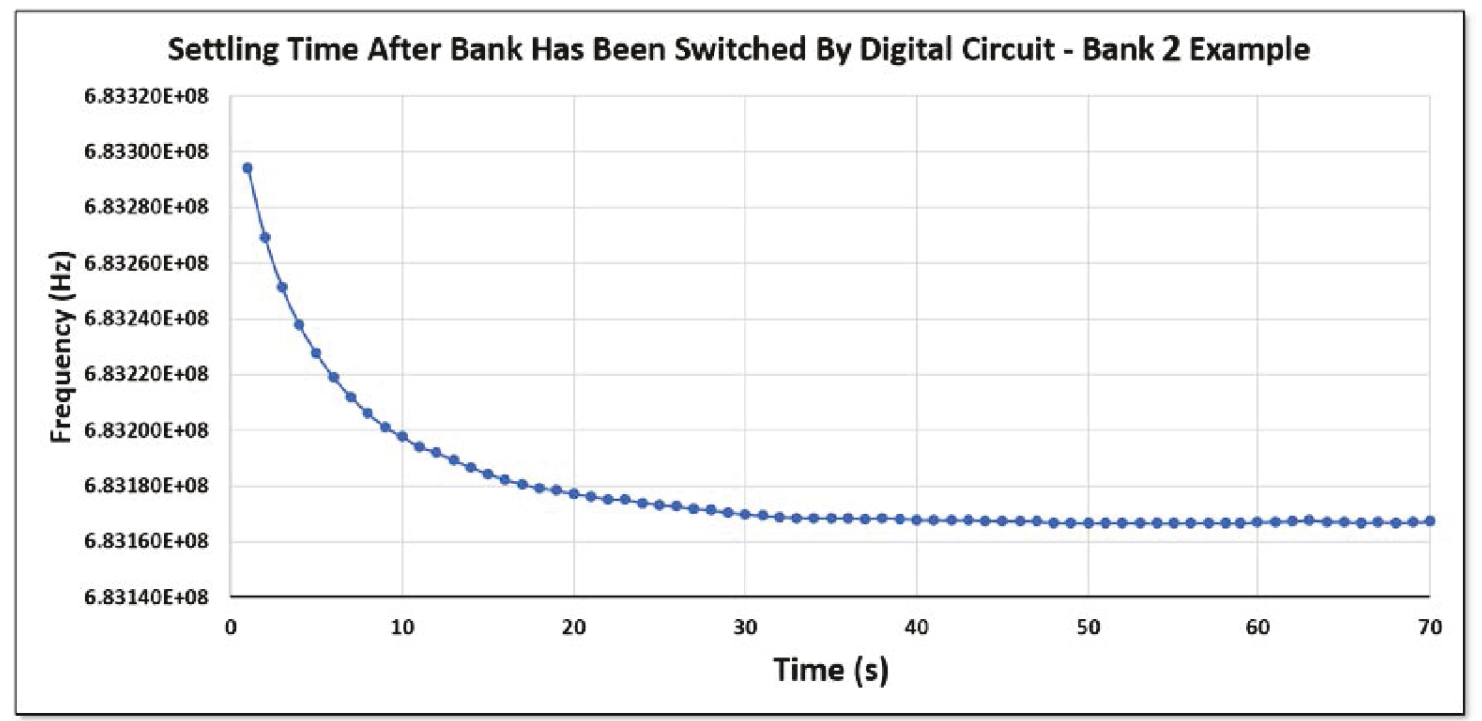

Figure 119: An example of frequency settling time required by banks in Bank Set 2 .

An example of a bank 3 frequency settling time after the bank is switched is shown in Figure 120. 


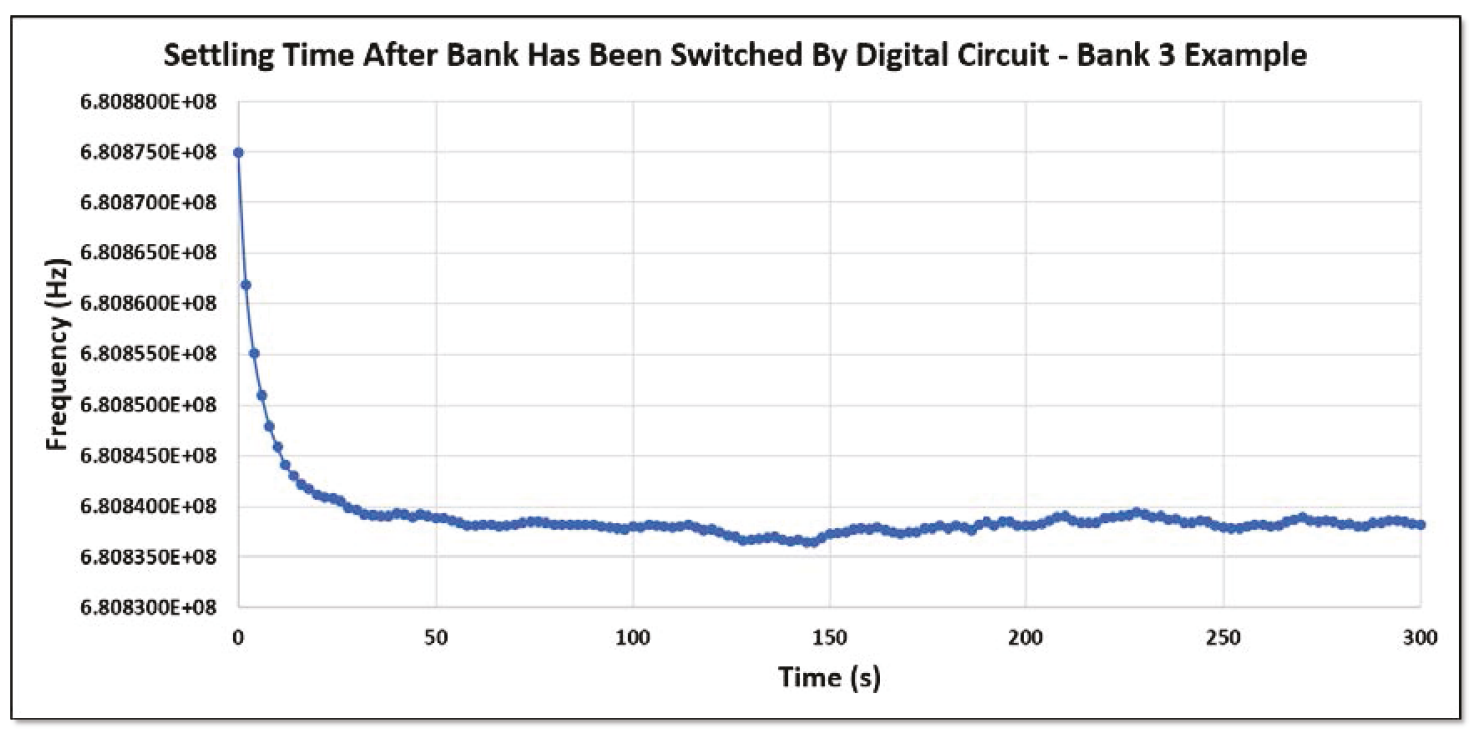

Figure 120: An example of frequency settling time required by banks in Bank Set 3 .

An example of bank 4 frequency settling time after the bank is switched is shown in Figure 121.

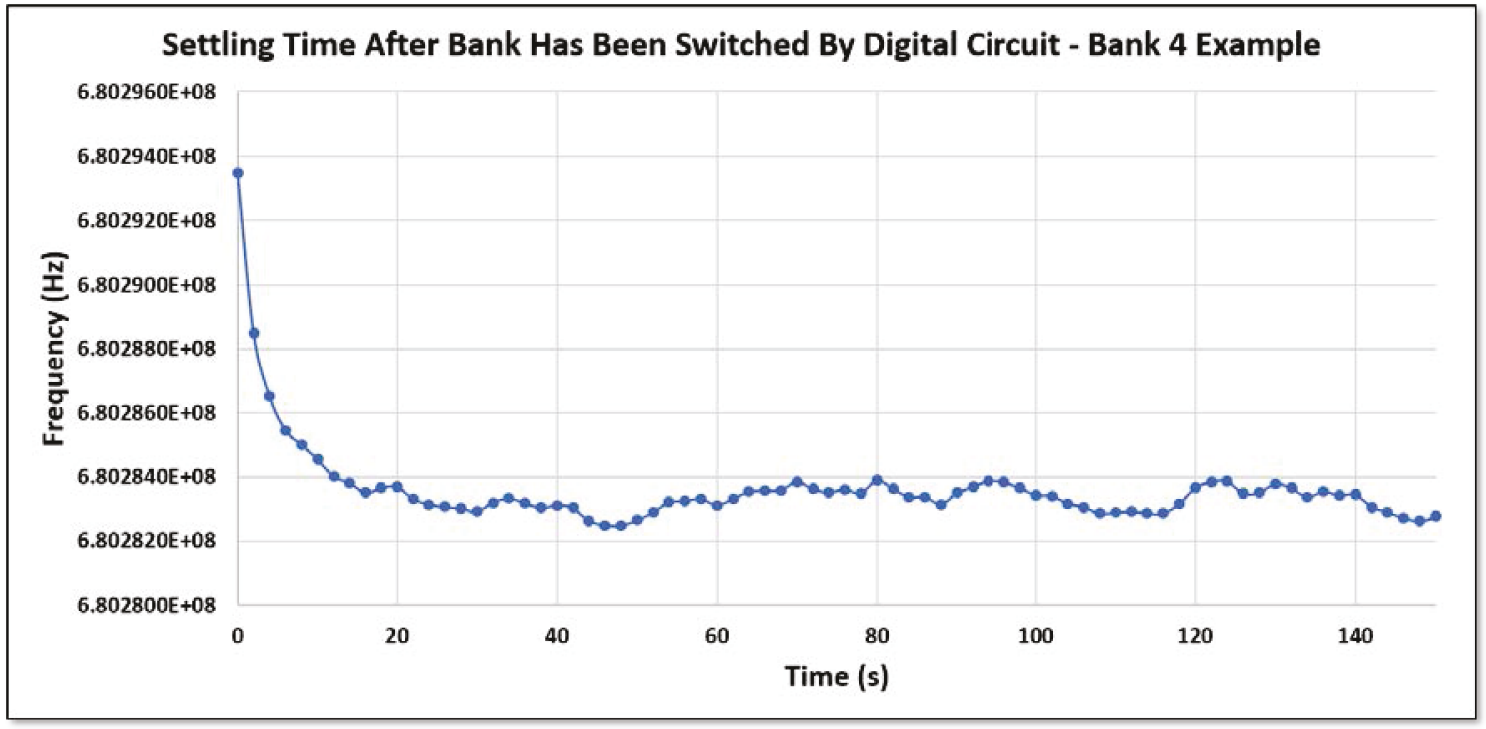

Figure 121: An example of frequency settling time required by banks in Bank Set 4.

\subsubsection{Multiple bank switch - cycling between Bank 1 and Bank 2}

The measured frequency response of cycling through Bank set 1 and Bank set 2 is shown in Figure 122. 


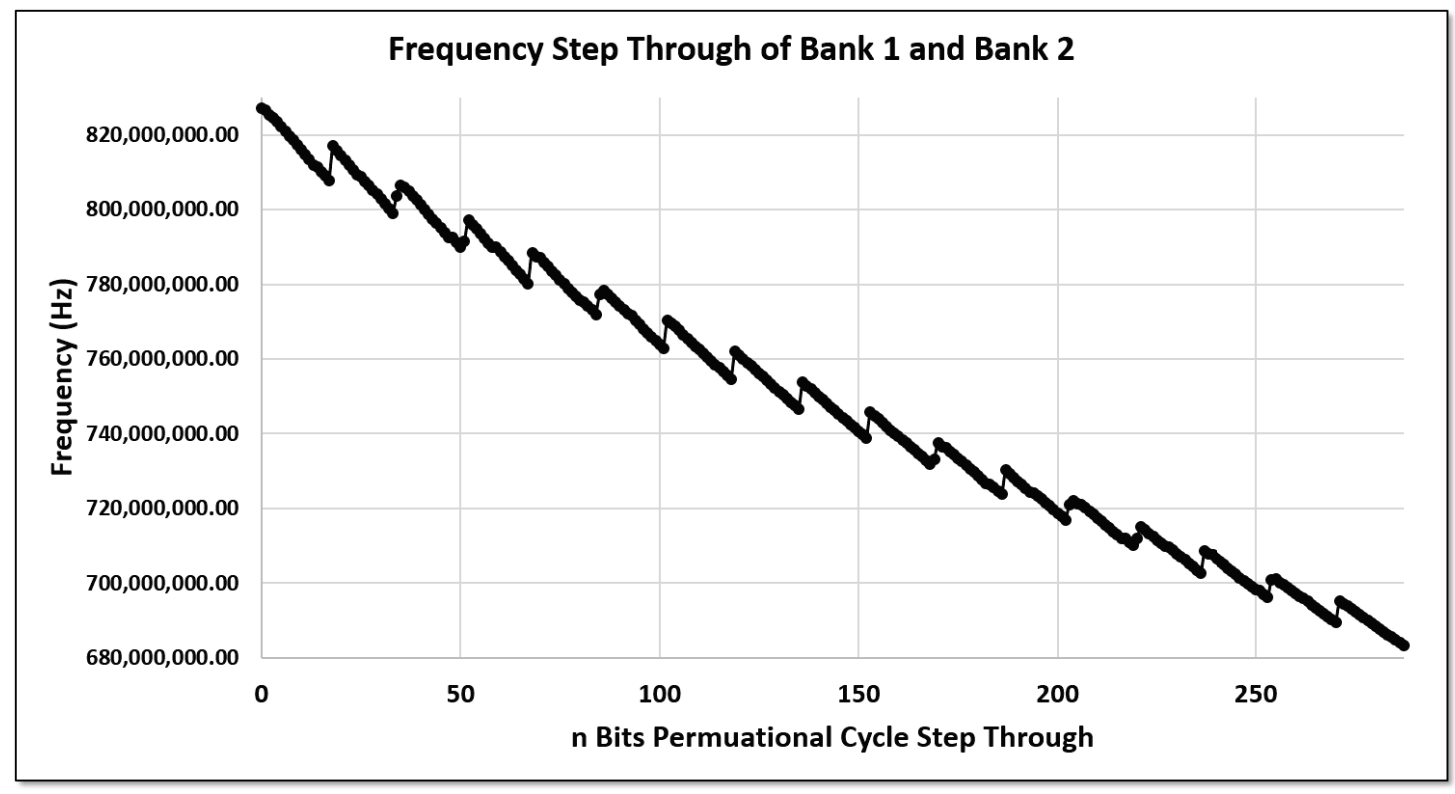

Figure 122: The frequency response of stepping through of Bank sets 1 and 2.

The resulting response when duplicate bank combinations (resulting in overlapped frequencies) are removed is shown in Figure 123. The average frequency step size was 8.4 MHz, with 138 steps (n bits) spanning from $6.62 \mathrm{GHz}$ to $5.47 \mathrm{GHz}$.

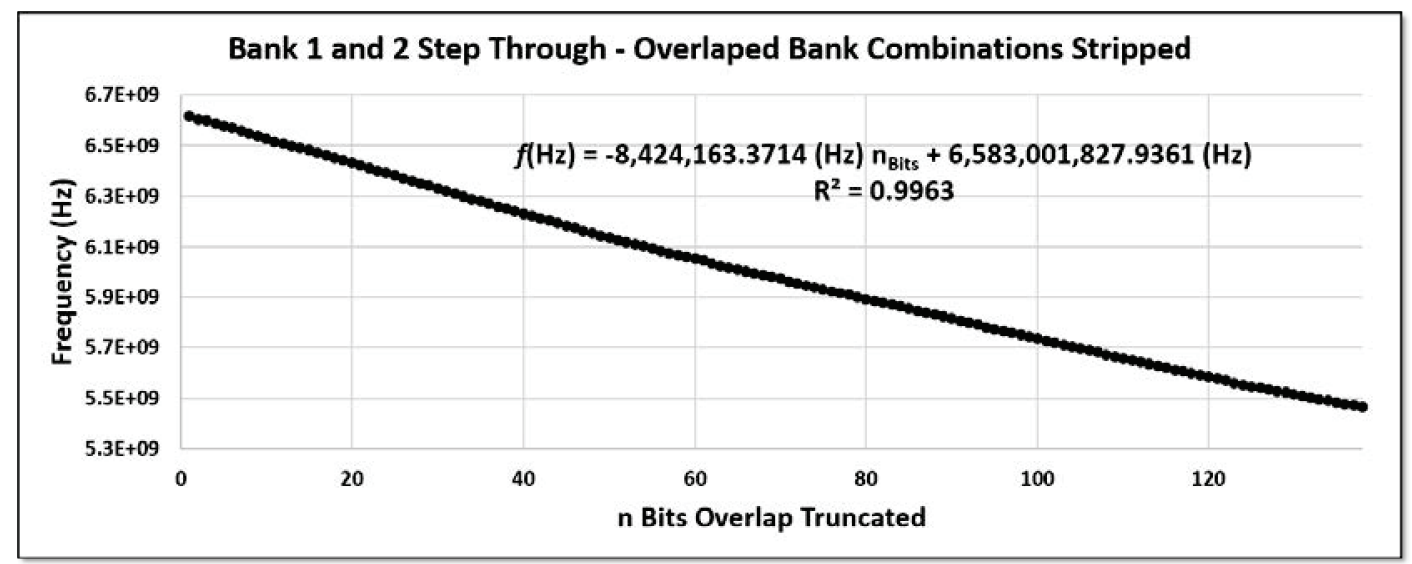

Figure 123: The frequency response of stepping through Bank sets 1 and 2 with duplicate bank combinations removed.

\subsubsection{Multiple bank switch - cycling between Bank sets 1, 2, and 3}

The measured frequency response of cycling through Bank sets 1, 2, and 3 is shown in Figure 124 . 


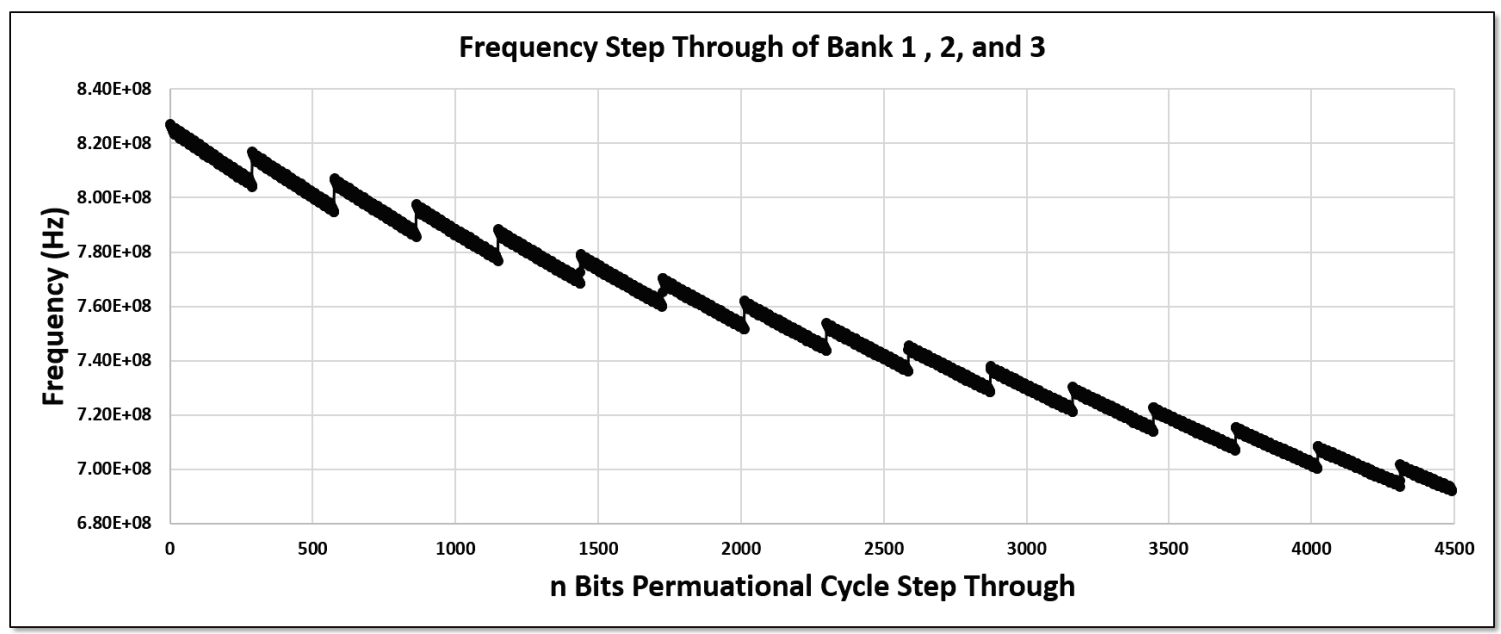

Figure 124: The frequency response of stepping through Banks sets 1, 2, and 3.

The resulting response when duplicate bank combinations are removed is shown in Figure 125. The average frequency step was $1.34 \mathrm{MHz}$, with 800 steps (n bits) spanning from 6.62 $\mathrm{GHz}$ to $5.47 \mathrm{GHz}$

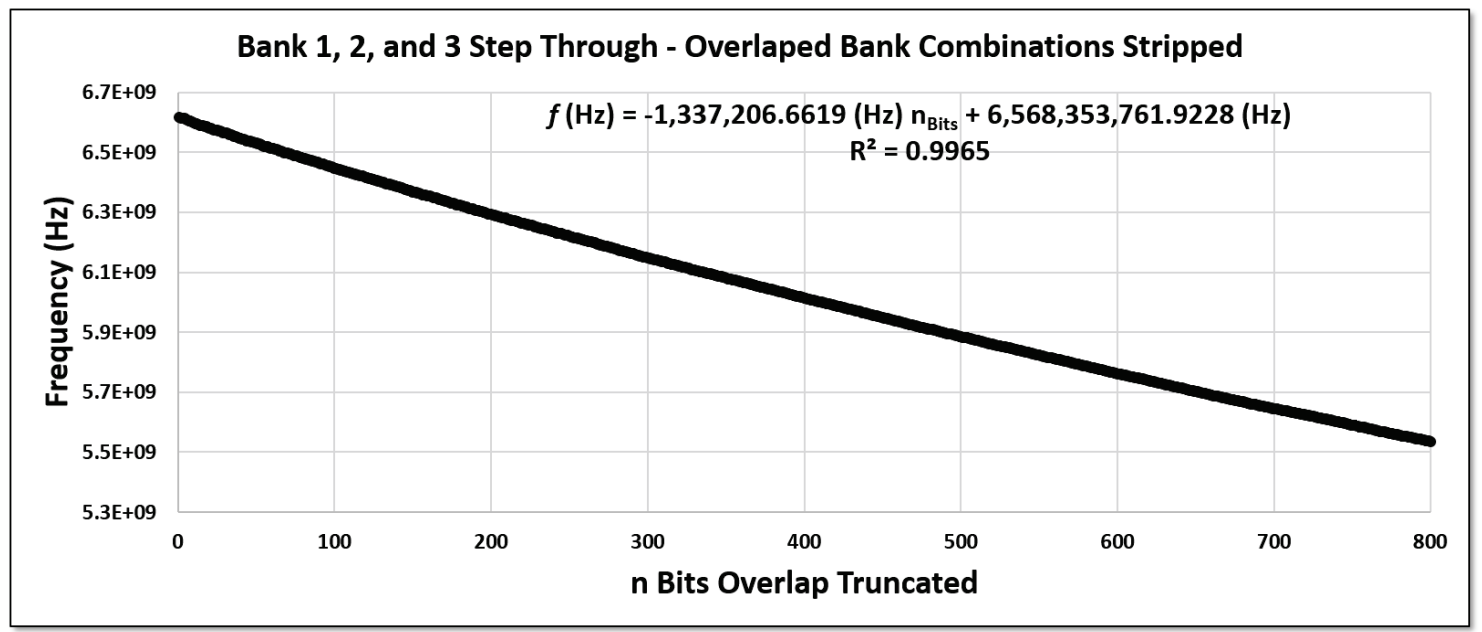

Figure 125: The frequency response of stepping through Bank sets 1,2, and 3 with duplicate bank combinations removed.

\subsubsection{Multiple bank switch - cycling between Bank sets 2, 3}

The measured frequency response of cycling through Bank sets 2 and 3 is shown in Figure 126. 


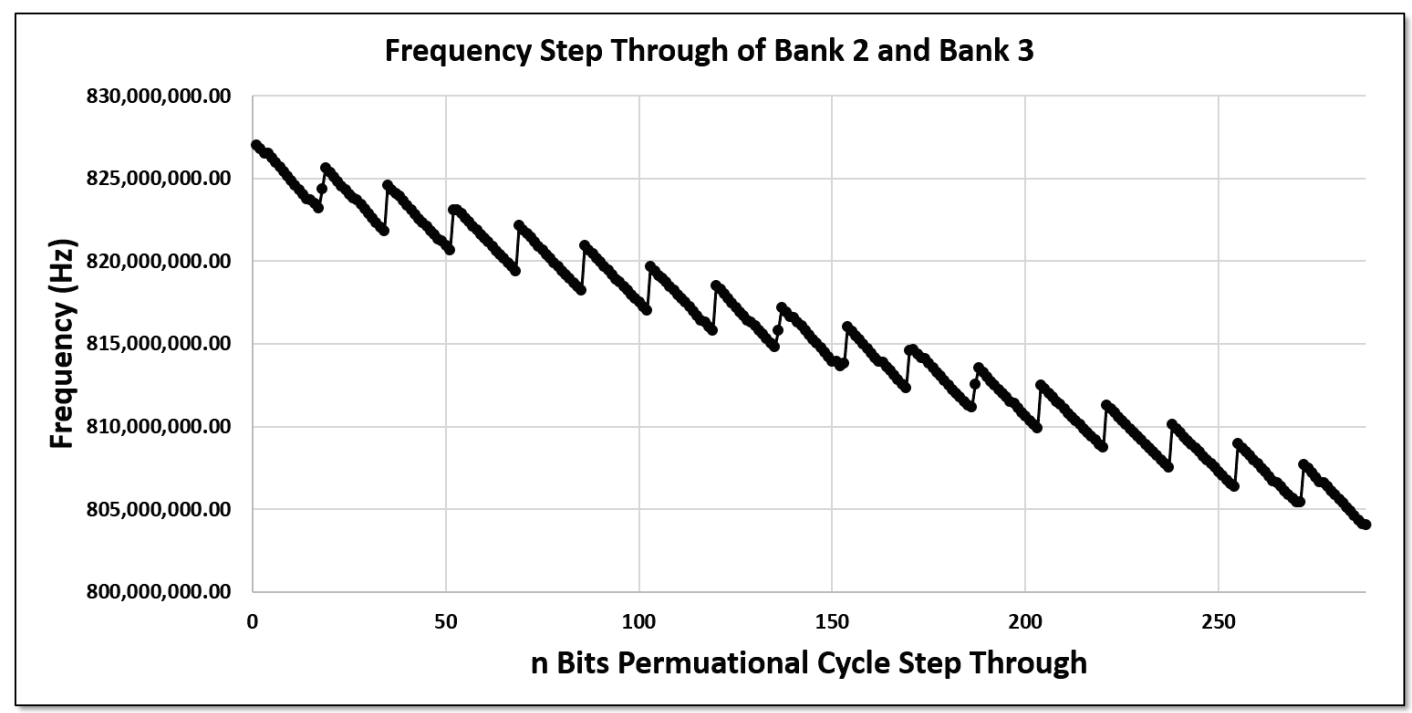

Figure 126: The frequency response of stepping through Bank sets 2 and 3.

The resulting response when duplicate bank combinations are removed is shown in Figure 127. The average frequency step size was $1.72 \mathrm{MHz}$, with 107 steps (n bits) spanning from $6.62 \mathrm{GHz}$ to $6.43 \mathrm{GHz}$.

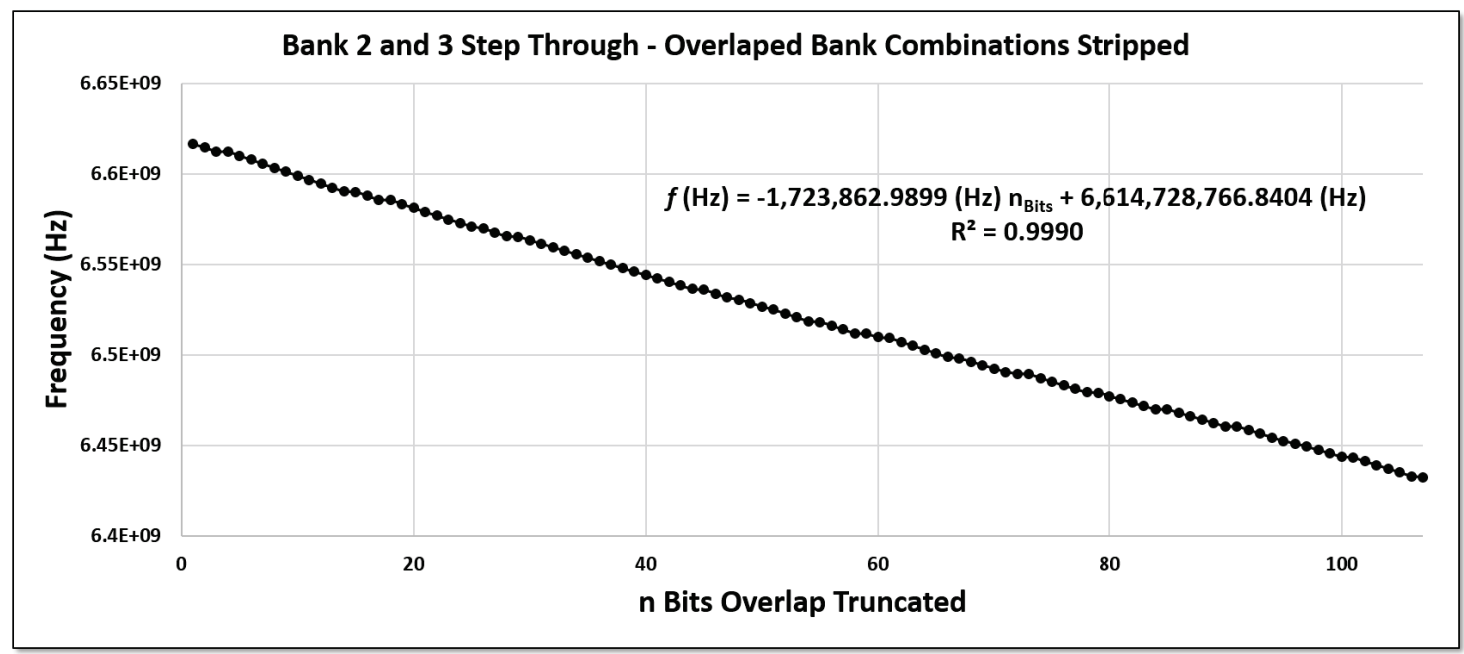

Figure 127: The frequency response of stepping through Bank sets 2 and 3 with duplicate bank combinations removed.

\subsubsection{Multiple bank switch - cycling between Bank sets 2, 3, 4}

The measured frequency response of cycling through Bank sets 2, 3, and 4 is shown in Figure 128. 


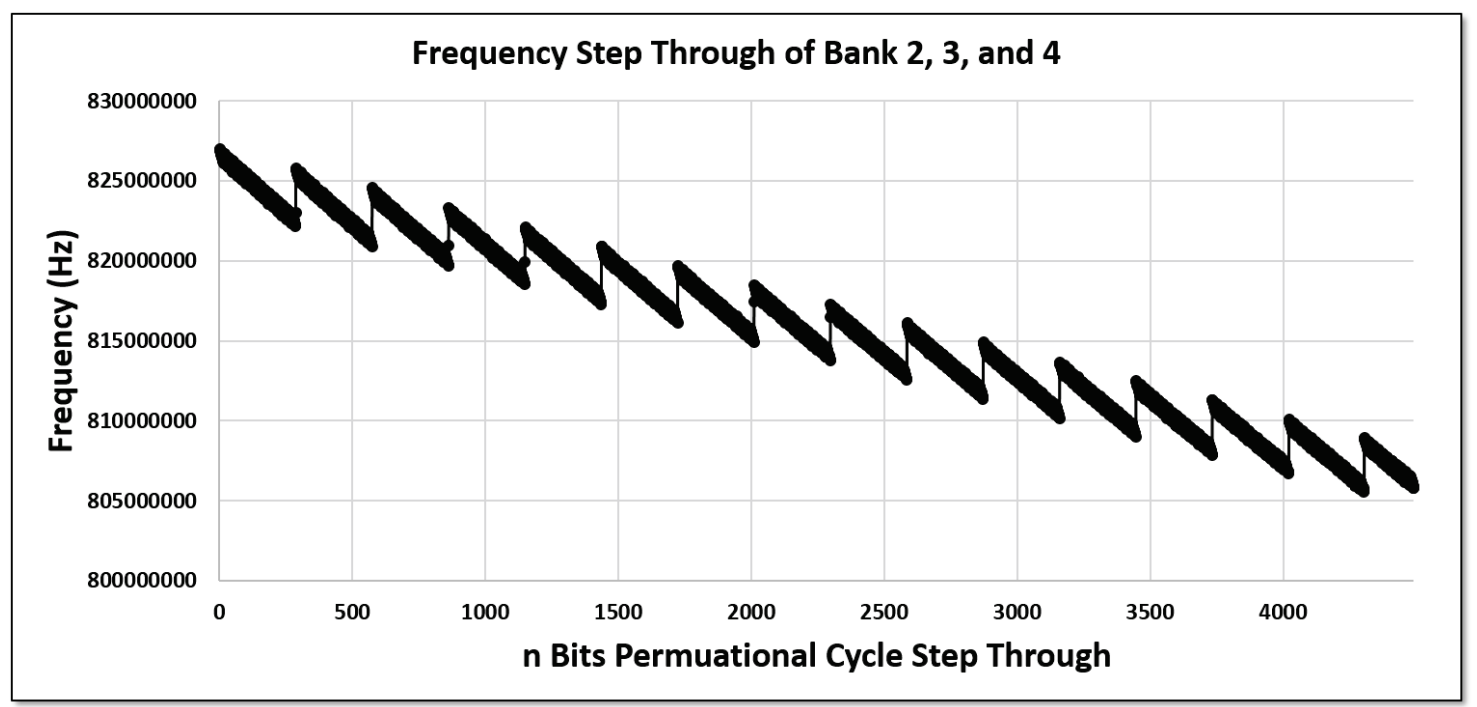

Figure 128: The frequency response of stepping through Bank sets 2, 3, and 4 .

The resulting response when duplicate bank combinations are removed is shown in Figure 129. The average frequency step size was $396.4 \mathrm{kHz}$ with 433 steps (n bits) spanning from $6.62 \mathrm{GHz}$ to $6.43 \mathrm{GHz}$.

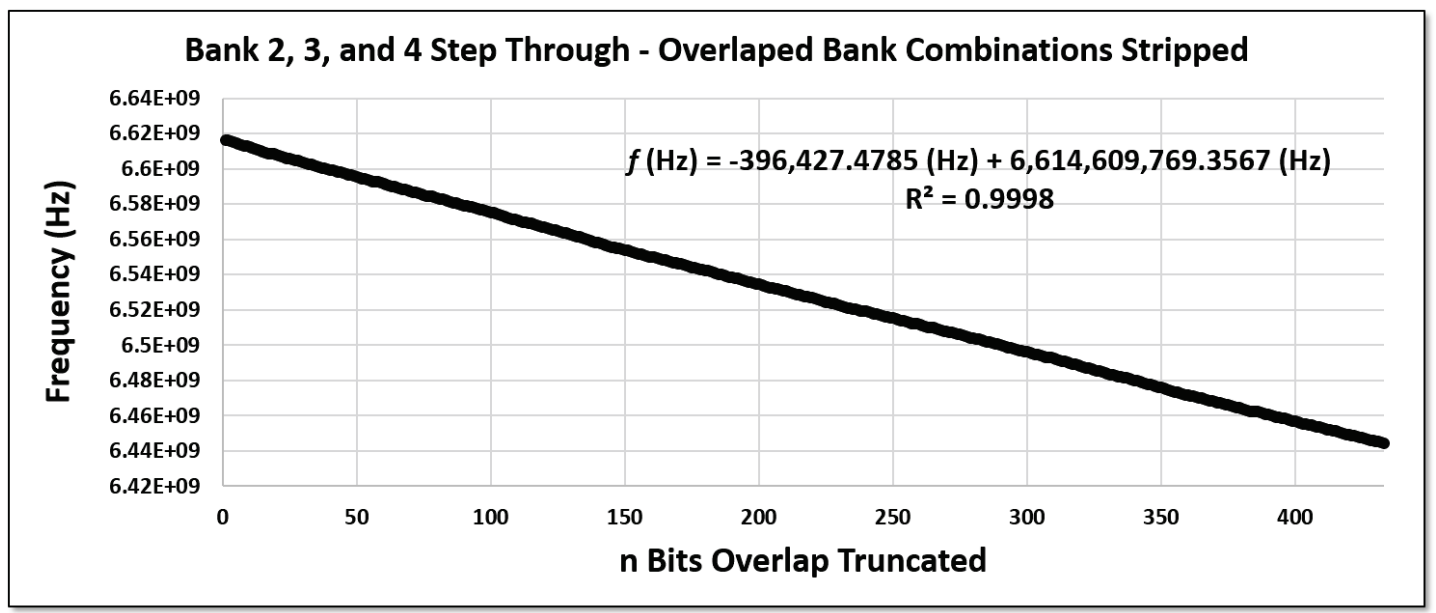

Figure 129: The frequency response of stepping through of Bank sets 2, 3, and 4 with duplicate bank combinations removed.

\subsection{Phase Noise Performance}

This section will present the measured phase noise performance of the oscillator. All phase noise measurement were done with the DCO in an open-loop configuration. 


\subsubsection{Phase Noise at upper and lower frequencies}

Measured phase noise when all the banks of the DCO are off is shown in Figure 130.

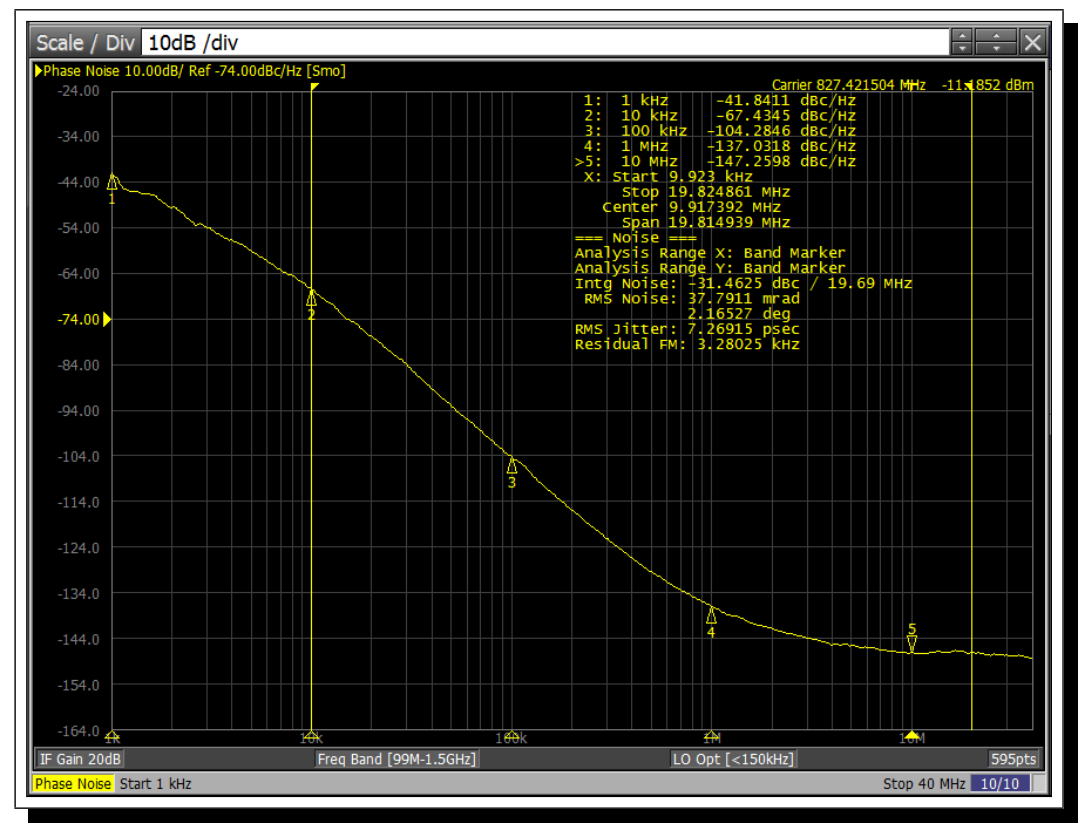

Figure 130: Phase noise when all banks are off. Carrier frequency was oscillator frequency divided by 8 due to 3 CMLs.

Measured phase noise when all the banks of the DCO are on is shown in Figure 131.

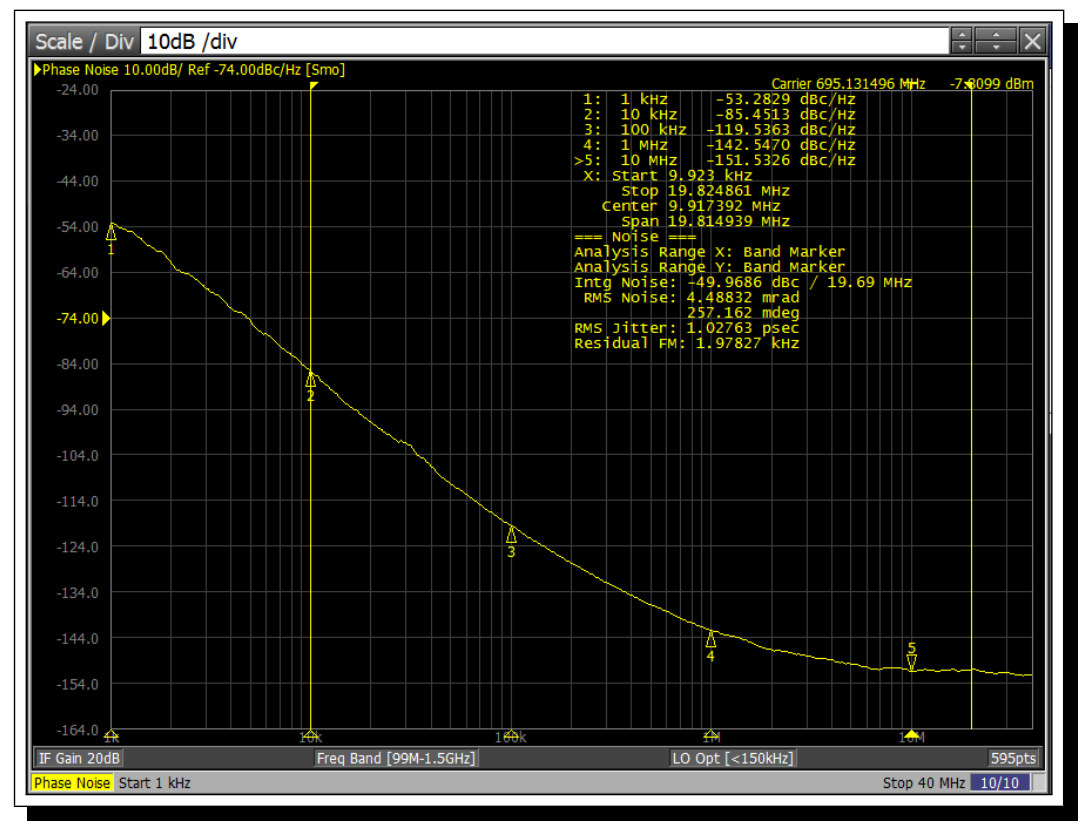

Figure 131: Phase noise when all banks are on. Carrier frequency was oscillator frequency divided by 8 due to 3 CMLs. 


\subsubsection{Phase noise over the frequency spectrum of oscillator}

The measured phase noise over the frequency spectrum of the DCO is plotted in Figure 132 and Figure 133.

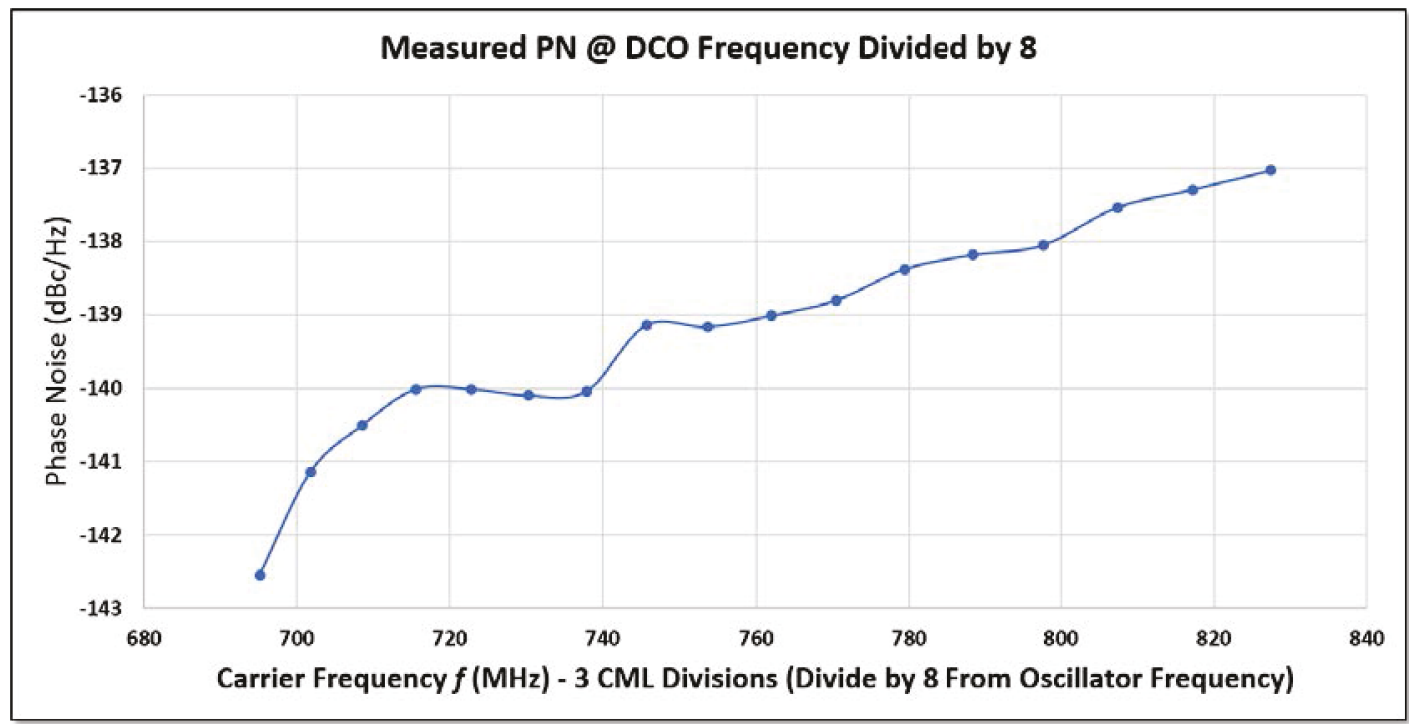

Figure 132: Measured PN over frequency range of DCO (Carrier frequency was oscillator frequency divided by 8 ).

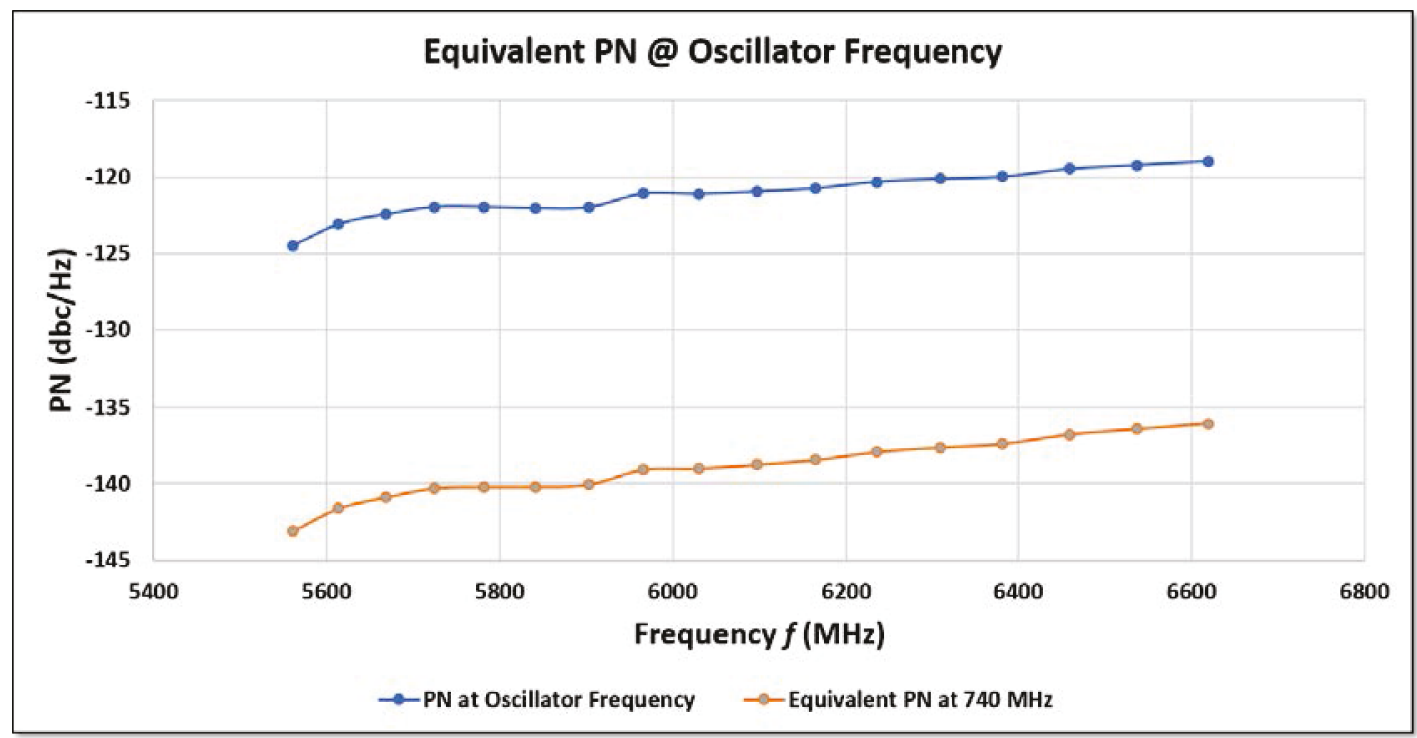

Figure 133: PN at oscillator frequency (from extrapolated measured results). Equivalent $\mathrm{PN}$ at $740 \mathrm{MHz}$ is also given as a reference. 


\subsubsection{Phase noise performance vs current consumption}

The measured phase noise vs measured current consumption (at oscillator frequency divided by 8 ) when all banks are off is shown in Figure 134.

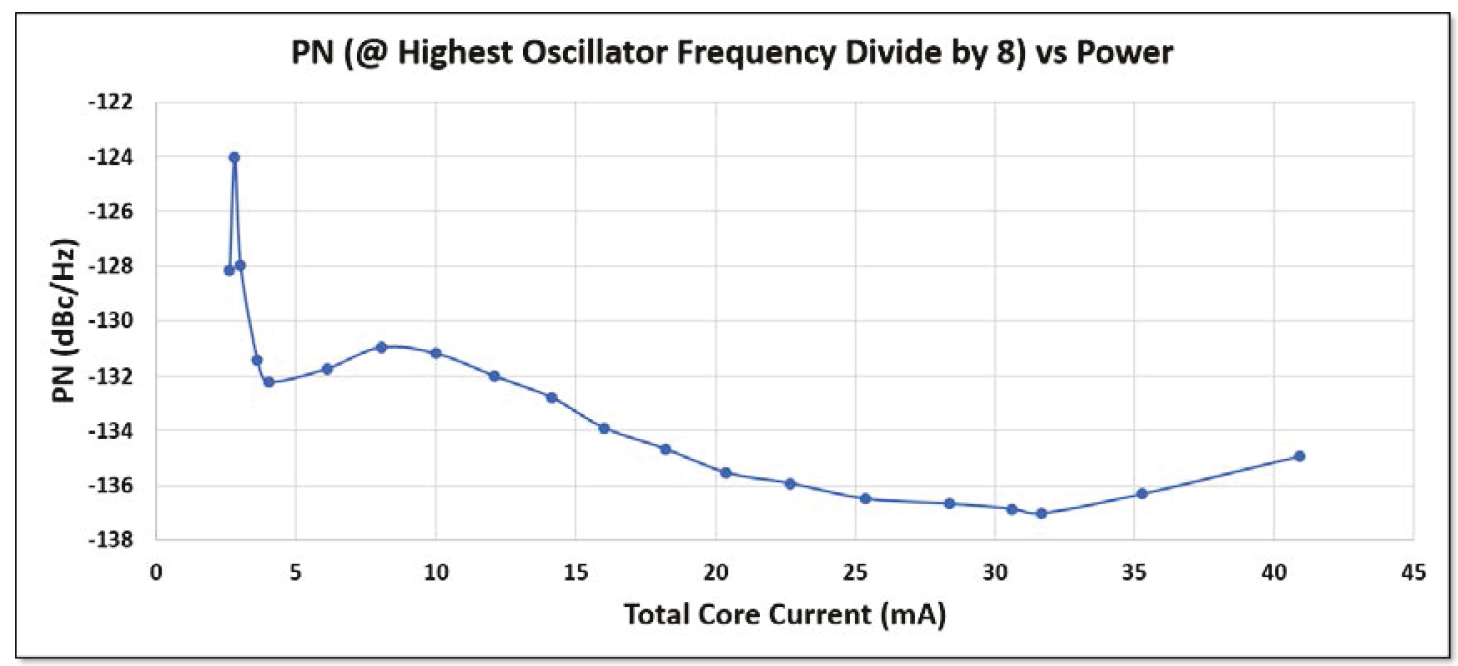

Figure 134: PN vs current consumption when all banks are off (carrier frequency was oscillator frequency divided by 8 ).

The measured phase noise vs measured current consumption (at oscillator frequency divided by 8 ) when all banks are on is shown in Figure 135.

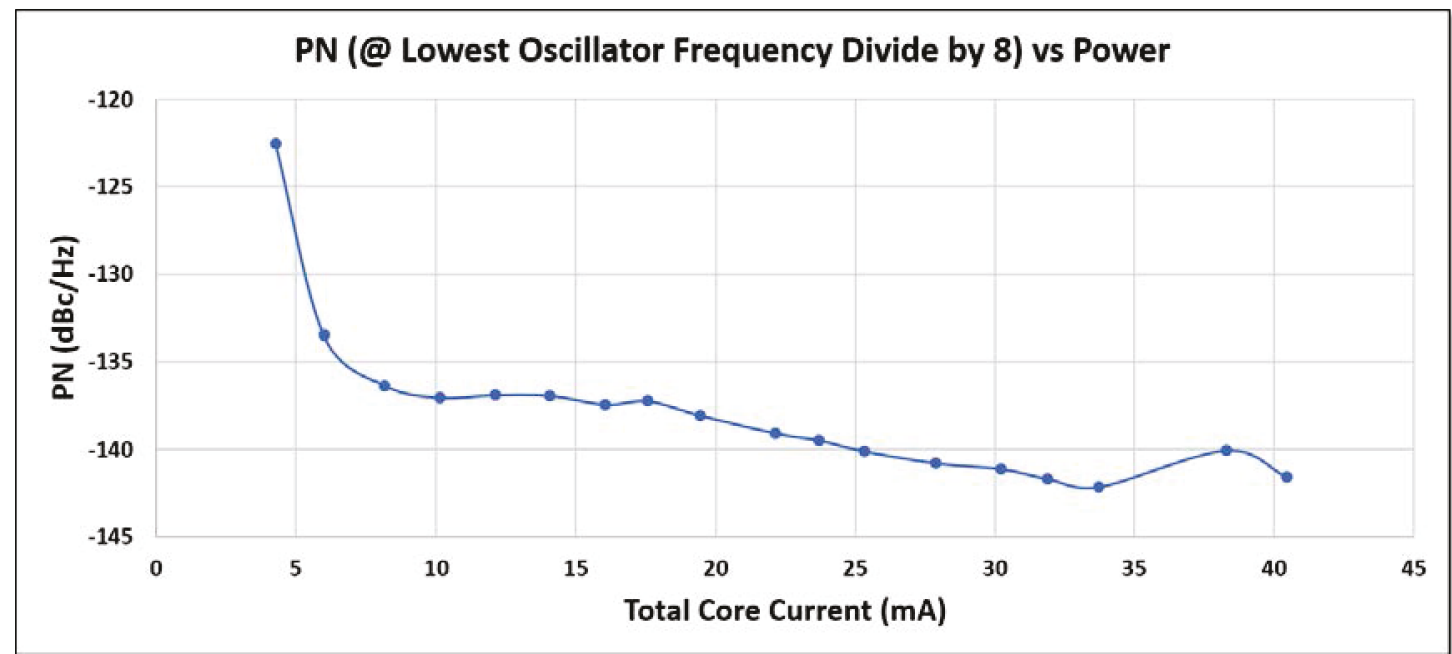

Figure 135: PN vs current consumption when all banks are on (carrier frequency was oscillator frequency divided by 8 ). 


\subsection{Figure of Merit}

The measured FoM of the oscillator for different power levels for when the all the banks are off and all the banks are on is shown in Figure 136 and Figure 137 respectively.

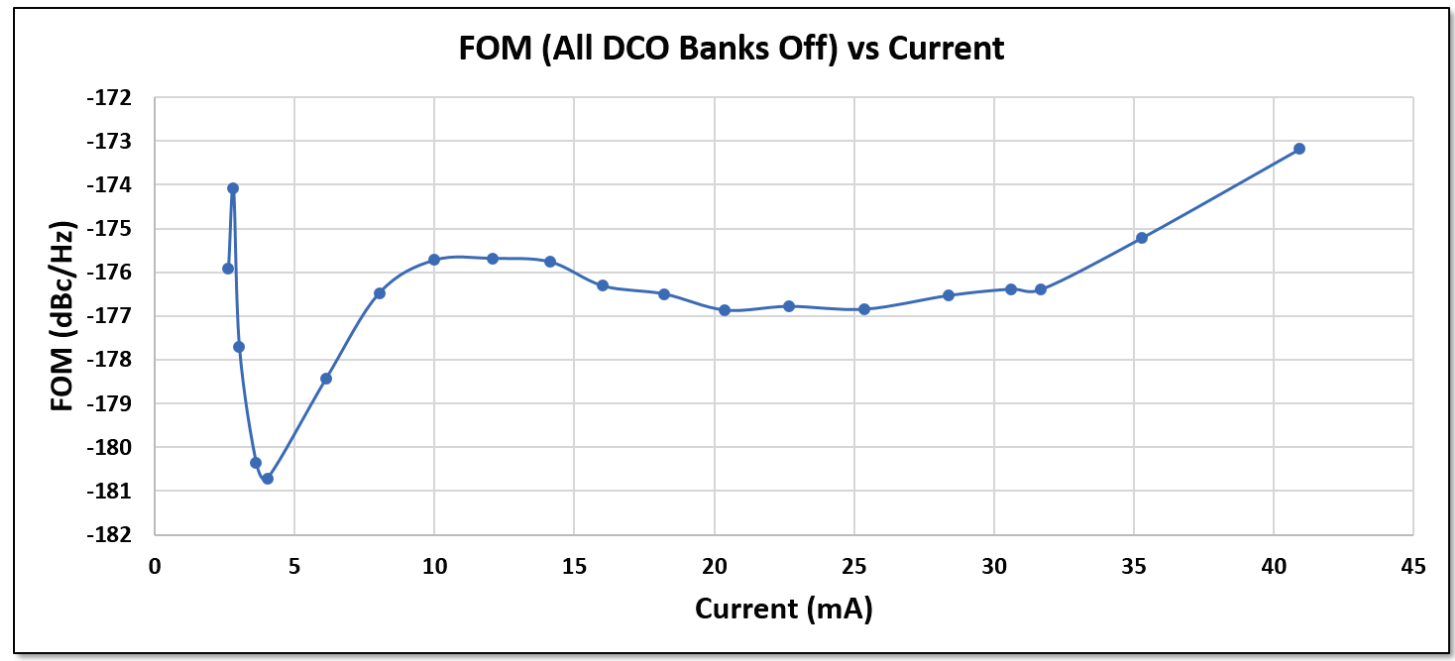

Figure 136: FoM of DCO vs various current consumption when all banks are off.

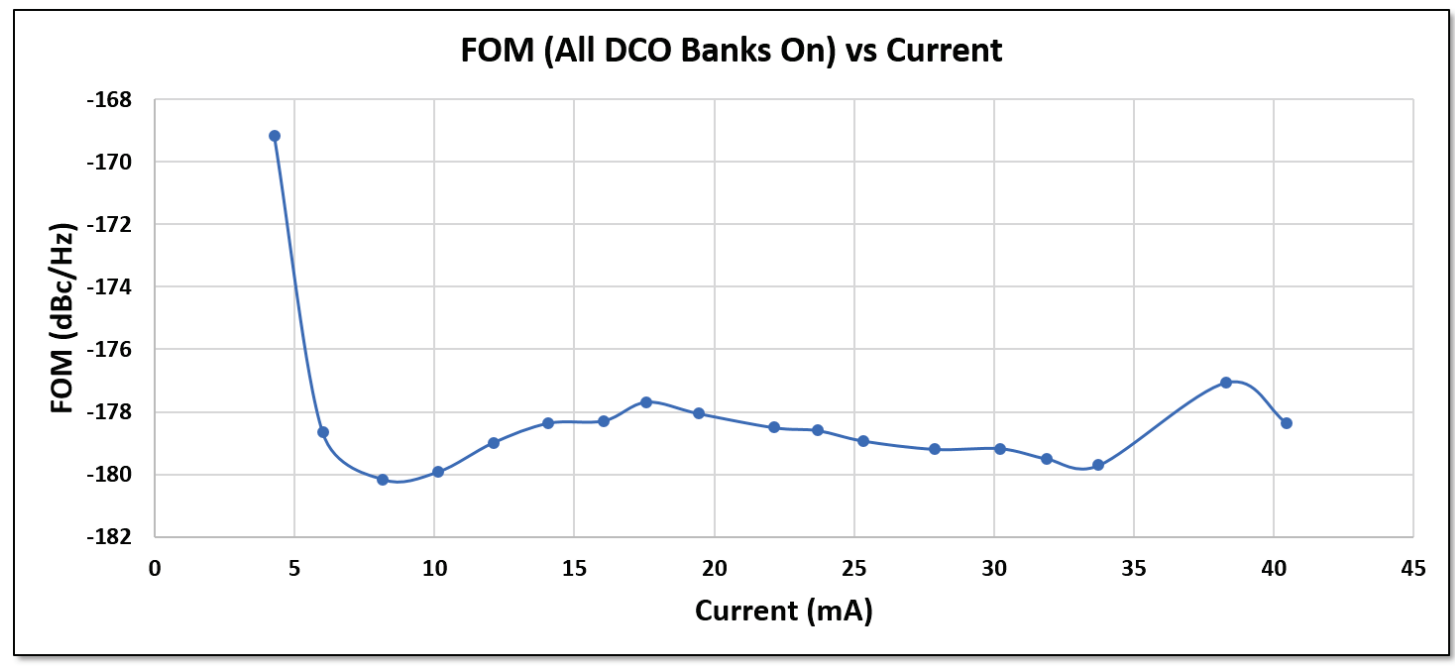

Figure 137: FoM of DCO vs various current consumption when all banks are on.

\subsection{Measured Tuning Range of Oscillator}

The measured tuning range of the entire oscillator frequency span (with all banks on and off) was:

Tunning Range $(\mathrm{TR}) \%=100 \cdot\left[\frac{2(6.656 \mathrm{GHz})-2(5.4456 \mathrm{GHz})}{6.656 \mathrm{GHz}+5.4456 \mathrm{GHz}}\right] \approx 20.00 \%$ 
The tuning range of just bank 1 (for theoretical comparison) was:

$$
\text { Tunning Range }(\mathrm{TR}) \%=100 \cdot\left[\frac{2(6.656 \mathrm{GHz})-2(5.5896 \mathrm{GHz})}{6.656 \mathrm{GHz}+5.5896 \mathrm{GHz}}\right] \approx 17.42 \%
$$

\subsection{Conclusion}

This chapter looked at the measured results of the DCO. It looked at the transient results when all the banks were on and off. The chapter also looked at the transient simulation of the ring oscillator designed to drive the digital circuit. The chapter then looked at the FFT response of the DCO. The measured frequency response of the DCO was from 6.66 $\mathrm{GHz}$ to $5.45 \mathrm{GHz}$. The chapter also looked at the phase noise performance of the DCO. The DCO had a phase noise of $-137.03 \mathrm{dBc} / \mathrm{Hz} @ 1 \mathrm{MHz}$ offset when all banks were off and $-142.55 \mathrm{dBc} / \mathrm{Hz} @ 1 \mathrm{MHz}$ offset when all banks were on. The FoM of the DCO was better than $-175 \mathrm{dBc} / \mathrm{Hz}$ throughout the frequency span of the DCO and for various power levels. The tuning range of the overall DCO was 20\%. The tuning range of only Bank set 1 was $17.42 \%$. 


\section{CHAPTER 10: ANALYSIS \& DISCUSSION}

This chapter will analyze the data presented in Chapter 9.

\subsection{Analysis of Transient Response}

The simulated transient response of divide by 8 and divide by 16 output from the CML was somewhat of a square wave (Figure 79) however the measured response on the oscilloscope was sinusoidal (Figure 102). This was possibly due to the parasitic of the trace, the balun, and the bandwidth of the oscilloscope (all collectively) acting as filters to filter out the high and low frequency components only leaving the original sinusoidal wave. See Figure 138 of a possible parasitic model of the differential to single ended signal reaching the oscilloscope.

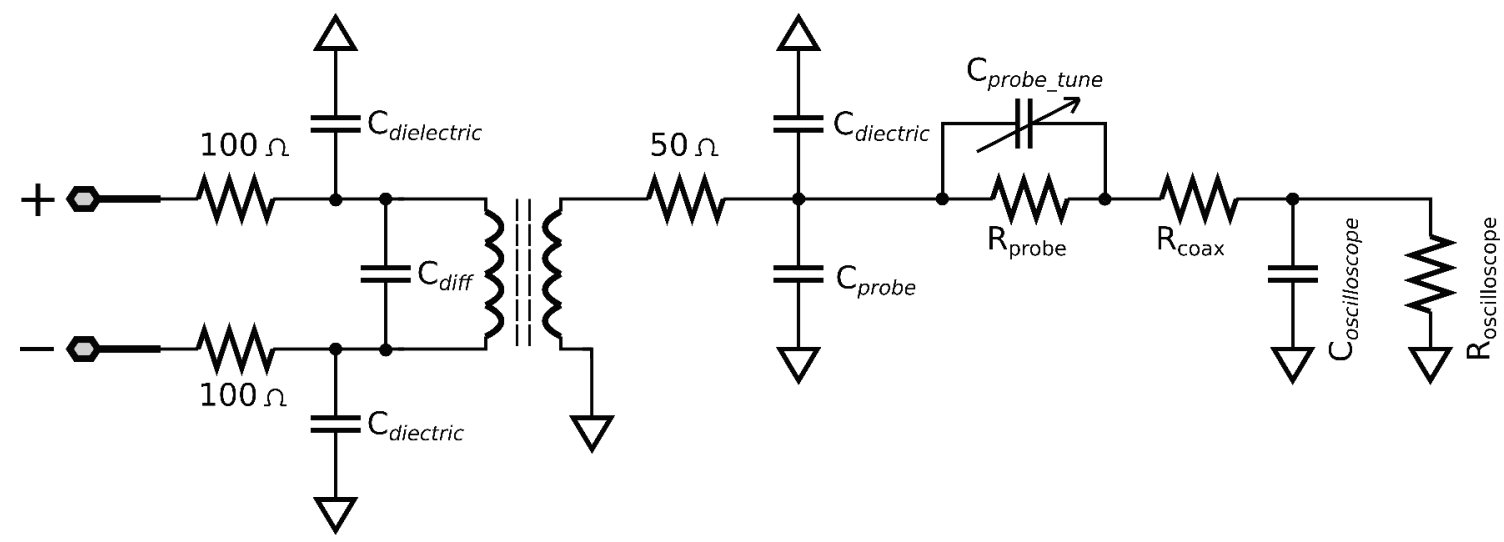

Figure 138: Simplified parasitic model between CML output and oscilloscope.

Figure 139 shows a quick simulation of the parasitic model of Figure 138, and Figure 140 shows an example of a test bench setup used to simulate the parasitic model. The input signal was $500 \mathrm{MHz}$ square wave clock, the output signal was a sinusoidal. 


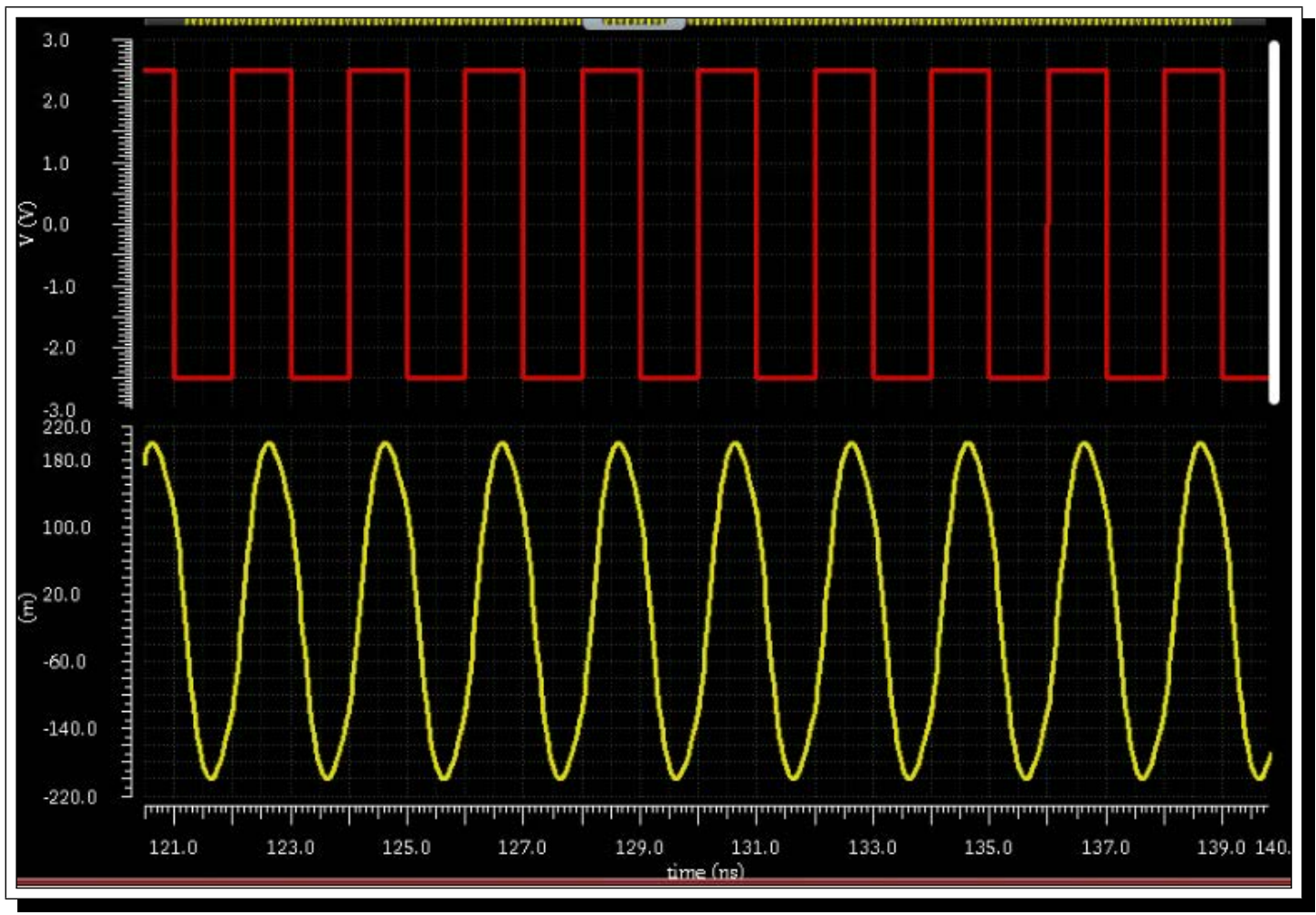

Figure 139: Test simulation of the parasitic model. Top signal emulates the signal from the CML, the bottom signal represents what could be observed on an oscilloscope.

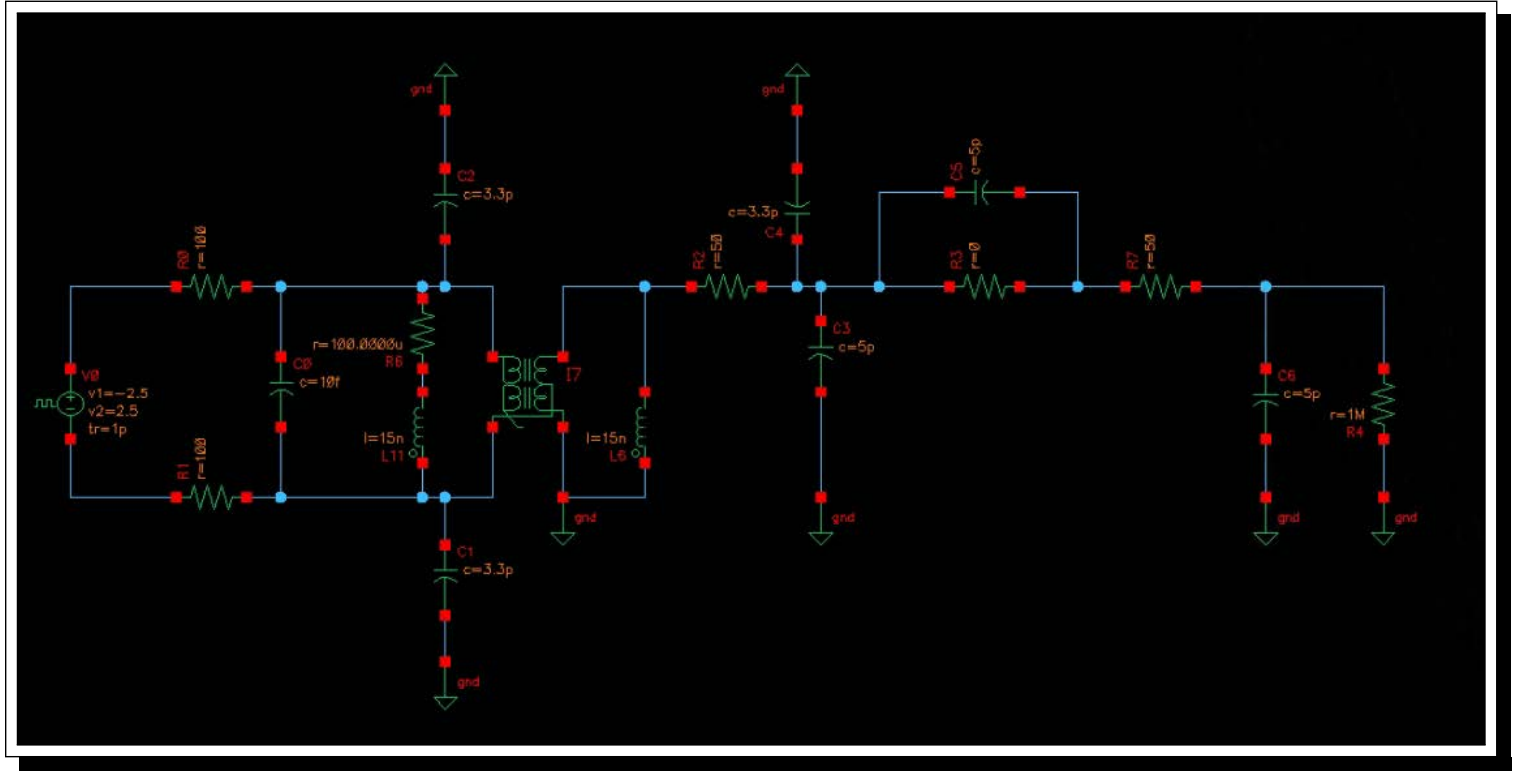

Figure 140: Test bench for simulating the parasitic model.

Rough estimates were used for the parasitic values; $3.3 \mathrm{pF}$ capacitance load for an inch of trace, total $15 \mathrm{pF}$ for the oscilloscope and oscilloscope probe, and an assumption of $10 \mathrm{fF}$ 
between the differential traces. $R_{\text {probe }}$ is $0 \Omega$ since the probe was bypassed when making the measurements. Inductors were put in parallel with an ideal balun to add inductance to the balun. A small resistor $(100 \mathrm{u} \Omega$ ) was placed in series with one of the inductors of the balun for simulation to converge. While the model may not be perfect, it could potentially give an insight as to why a sinusoid was observed on the oscilloscope.

\subsection{Analysis of Frequency Response}

The simulated frequency response was from $7.14 \mathrm{GHz}$ to $5.74 \mathrm{GHz}$ however the measured response was from $6.66 \mathrm{GHz}$ to $5.45 \mathrm{GHz}$. The upper frequency dropped by almost 480 $\mathrm{MHz}$ and the lower frequency dropped by almost $290 \mathrm{MHz}$. This was probably due to the fact that the bank circuits were not fully simulated using EMX during the design process due to computational limits of port use. The metal between the bank capacitors and switch transistor were assumed small enough that it was modelled as an ideal wire; however having $200+$ banks, the additive parasitic could have been significant enough to reduce the frequency of the oscillator. Figure 141 shows capacitance simulation of Bank 1 with (96.7 $\mathrm{fF})$ and without Calibre $\mathrm{RC}(\mathrm{C}+\mathrm{CC})$ extraction $(94.9 \mathrm{fF})$ when the bank is on at $6 \mathrm{GHz}$.

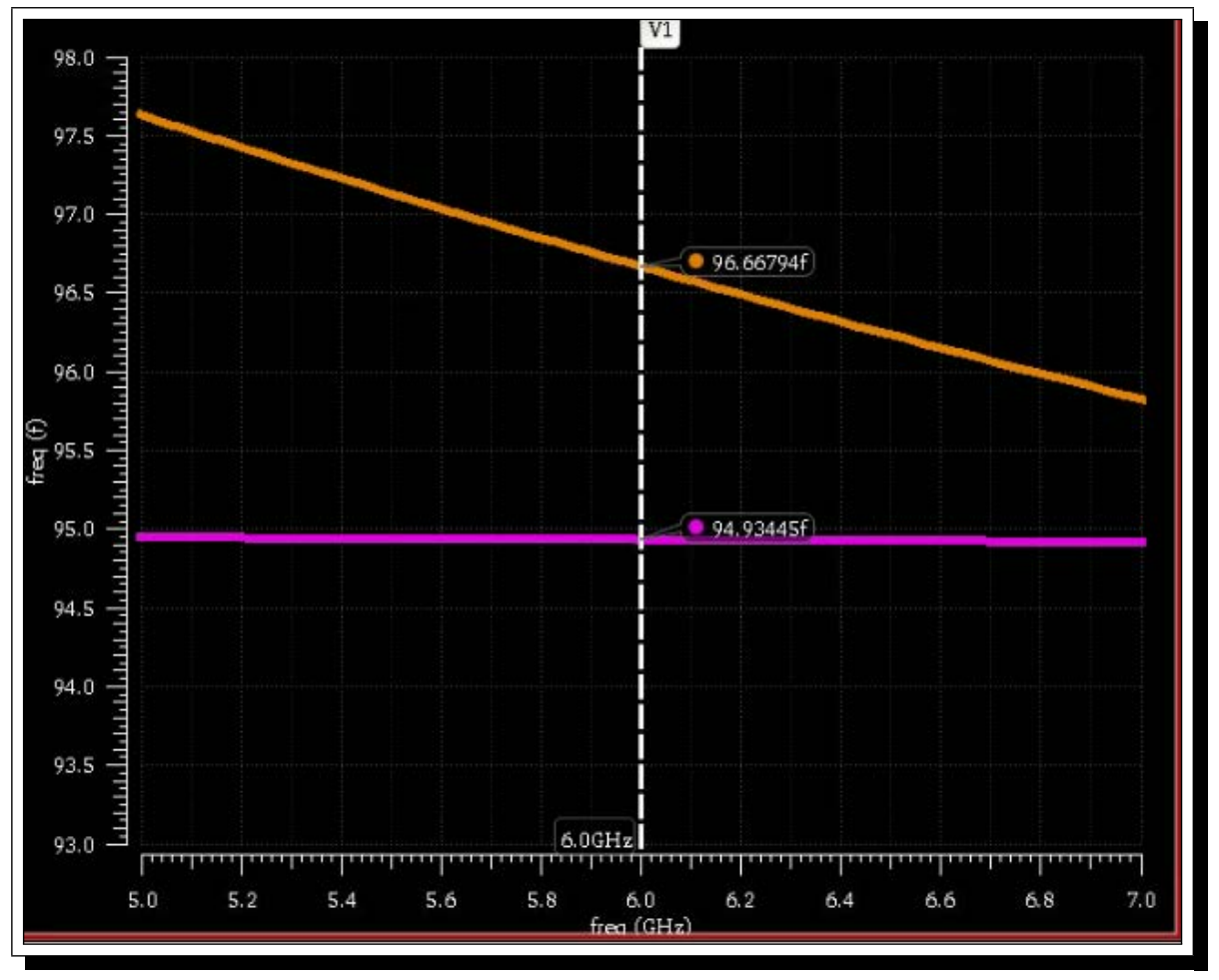

Figure 141: Simulation of Bank 1 with (orange) and without RC extraction (purple).

While a difference of $1.8 \mathrm{fF}$ may not be much for one bank, when $200+$ banks is considered, 
it adds up significantly. Consider an oscillator with core total of $470 \mathrm{pH}$ inductance and 1.5 $\mathrm{pF}$ of capacitance:

$$
f=\frac{1}{2 \pi \sqrt{470 p H \cdot 1.5 p F}}=6 \mathrm{GHz}
$$

And now consider the previous oscillator with an additional parasitic of $1.8 \mathrm{fF}$ for 64 banks (the number of banks on the drain side):

$$
f=\frac{1}{2 \pi \sqrt{470 p H \cdot(1.5 p F+64 \times 1.8 f F)}}=5.78 \mathrm{GHz}
$$

The added (extracted) parasitic has dropped the frequency by $220 \mathrm{MHz}$ (using extracted measurement for when bank was on). In comparison the measured frequency drop of the oscillator designed for this thesis was $290 \mathrm{MHz}$ when all banks were on. Of course the analysis is a crude estimate, not all banks will have the same additive parasitic value. The extracted parasitic from $100+$ banks on the source side will also contribute to lowering the frequency however they will be minimized due to capacitive degeneration; nevertheless due to the sheer number of banks, the total parasitic value transformed to the drain side will still be significant.

Another factor that could have played a role in decreasing the measured frequency response from the simulated response was the metal fills that were placed around the inductor and banks of the core of the oscillator. The metal fills (required for kit density checks) added additional parasitic capacitance (hence reducing the frequency). Again due to computational simulator limits, it was impractical to simulate such extractions with metal fills. See Figure 142 for example of metal fills around the core of the oscillator and Figure 143 and Figure 144 for a possible abstract model of the parasitic. 


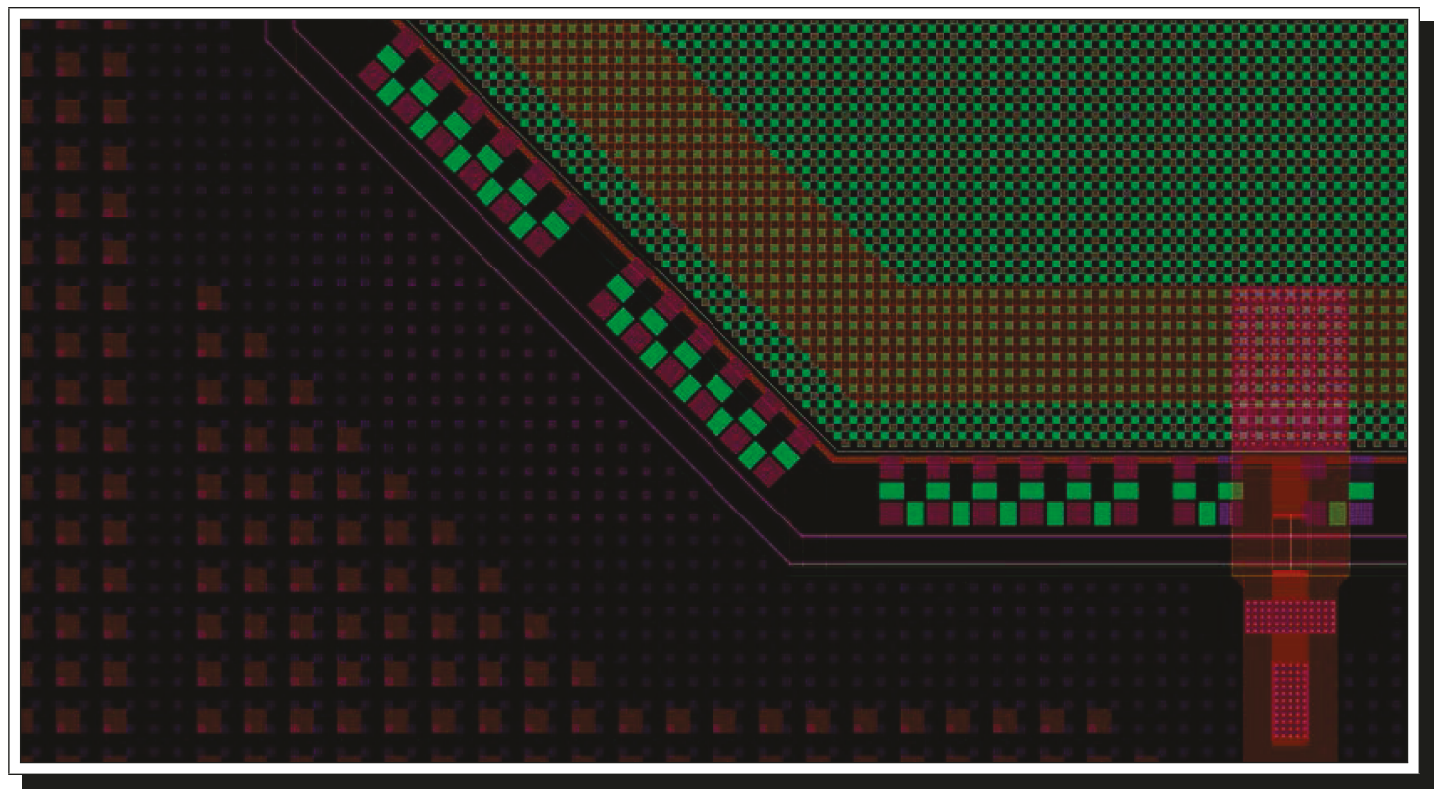

Figure 142: Metal fills around the inductor of the core of the oscillator due to kit density requirements.

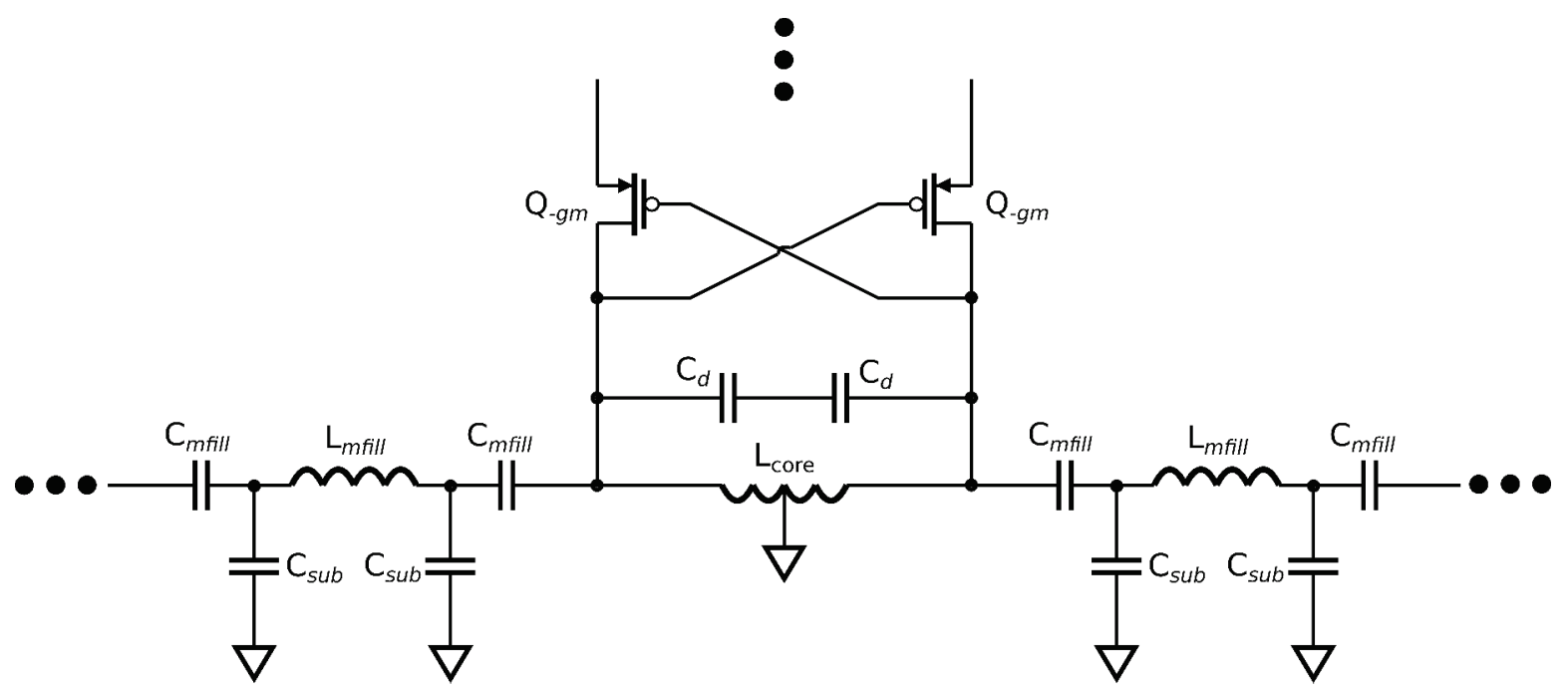

Figure 143: Parasitic due to metal fill (that might be affecting the core frequency of the DCO.)

A simplistic equivalent LC core model with parasitic of Figure 143 is shown in Figure 144. 

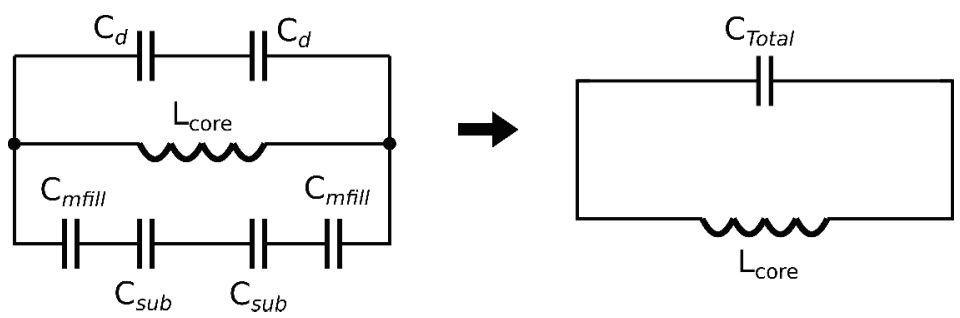

Figure 144: LC core with parasitic included.

The frequency span of the simulated results was $1.4 \mathrm{GHz}$ while the measured results was 1.07 GHz. The frequency span dropped by $23.6 \%$, this is expected since the same capacitor banks will switch a smaller frequency ratio for lower core frequency if the core capacitance increases due to parasitic. The proof for this is beyond the scope of the thesis however assuming this identity is true:

$$
\begin{aligned}
& b>a ; \\
& d>c ; \\
& \frac{1}{\sqrt{a+c}}-\frac{1}{\sqrt{a+d}}>\frac{1}{\sqrt{b+c}}-\frac{1}{\sqrt{b+d}}
\end{aligned}
$$

then it simply follows:

$$
\begin{aligned}
& C_{\text {measured }}>C_{\text {simulation }} \\
& C_{\text {bank }_{\text {on }}}>C_{\text {bank }_{\text {off }}} \\
& \frac{1}{2 \pi \sqrt{C_{\text {sim }}+n C_{\text {off }}}}-\frac{1}{2 \pi \sqrt{C_{\text {sim }}+n C_{\text {on }}}}>\frac{1}{2 \pi \sqrt{C_{\text {meas }}+n C_{\text {on }}}}-\frac{1}{2 \pi \sqrt{C_{\text {meas }}+n C_{\text {off }}}} \\
& f_{\text {max }_{\text {sim }}}-f_{\text {min }_{\text {sim }}}>f_{\text {max }_{\text {meas }}}-f_{\text {min }_{\text {meas }}} \\
& \Delta f_{\text {simulation }}>\Delta f_{\text {measured }}
\end{aligned}
$$

Another way to realize it is to visualize the frequency curve with respect to capacitance (shown in Figure 145). As can be seen in Figure 145, the frequency span has shrunk for the measured frequency even though the span of the capacitance is the same for both simulation and measurement. 


\section{Frequency vs Capacitance}

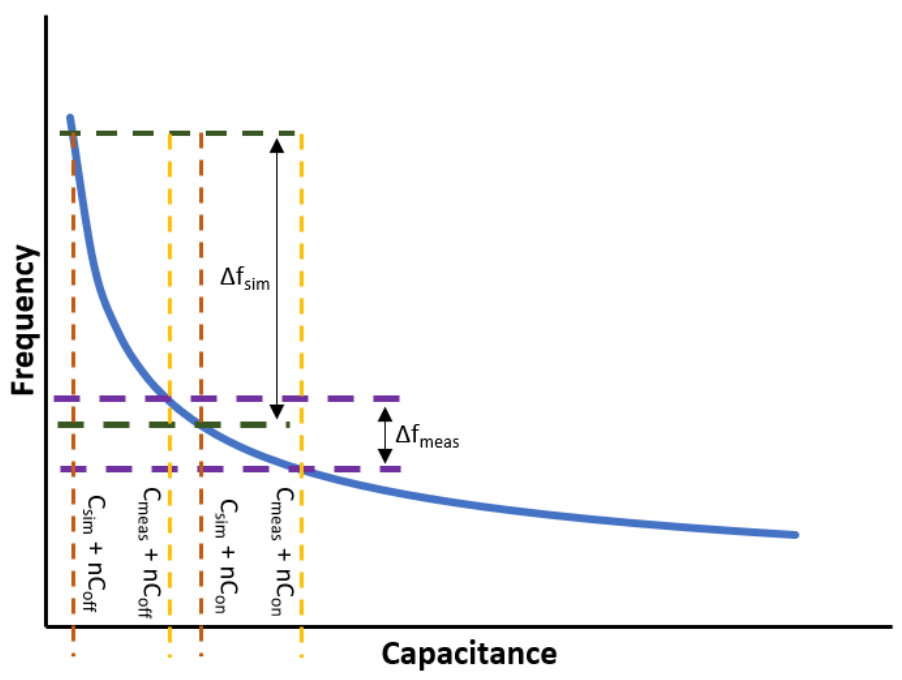

Figure 145: LC curve showing the frequency span between simulation and measurement. ***Note: the image has been significantly pronounced and exaggerated to get the point across and is not to scale.

The tuning range was almost the same between simulation $(21.7 \%)$ and measured results $(20.0 \%)$. This is also expected if:

$$
\begin{aligned}
& C_{\text {simulation }}<<n C_{\text {bank }_{\text {off }}} \\
& C_{\text {measured }}<<n C_{\text {bank }_{\text {off }}}
\end{aligned}
$$

then:

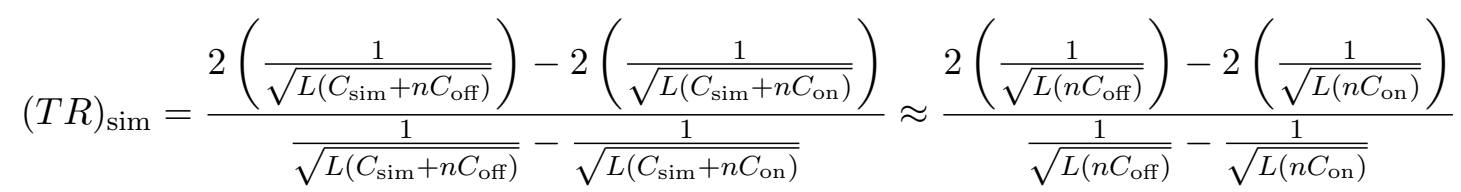

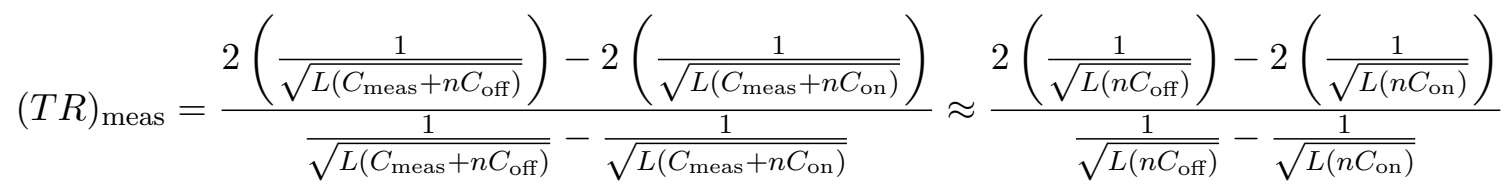

$$
\begin{aligned}
& \therefore(T R)_{\mathrm{sim}} \approx(T R)_{\text {meas }}
\end{aligned}
$$

Table 5 summarizes and compares the frequency response between the theoretical, simulated and measured response for Bank set 1 of the DCO. 
TABLE 5: Frequency Response Comparison Between Theoretical, Simulated, and Measured DCO.

\begin{tabular}{|c|c|c|c|c|c|}
\hline Spec & Theory & Simulation & Error & Measured & Error \\
\hline Upper Frequency & $7.17 \mathrm{GHz}$ & $7.14 \mathrm{GHz}$ & $0.418 \%$ & $6.66 \mathrm{GHz}$ & $7.113 \%$ \\
\hline Lower Frequency & $5.94 \mathrm{GHz}$ & $5.90 \mathrm{GHz}$ & $0.673 \%$ & $5.59 \mathrm{GHz}$ & $5.892 \%$ \\
\hline Frequency Span & $1.23 \mathrm{GHz}$ & $1.24 \mathrm{GHz}$ & $0.813 \%$ & $1.07 \mathrm{GHz}$ & $13.01 \%$ \\
\hline Tuning Range & $18.76 \%$ & $19.02 \%$ & $1.386 \%$ & 17.47 & $6.876 \%$ \\
\hline
\end{tabular}

\subsubsection{Frequency Response of Bank 5 to Bank 11}

The DCO did not respond properly to the tuning steps of Bank set 5; and Bank set 6 to 11 responded like Bank set 5. This could be due to the fact that the DCO resolution has reached its limit, or the parasitic around the banks could be overshadowing the bank capacitance. If the parasitic around the banks is overshadowing the bank capacitance then it could explain why Bank Set 5 was not fully linear even though some linearity was observed; and why Bank set 6 to 11 behaved like Bank set 5. The jitter performance of the DCO or the measuring equipment could have also limited the observable DCO resolution.

\subsection{Linearity Analysis}

A comparison of the linearity of Bank set 1 between prediction, simulation, and measured results is summarized in Table 6 .

TABLE 6: Linearity Comparison Between Theoretical, Simulated, and Measured Results.

\begin{tabular}{|c|c|c|c|c|c|}
\hline Spec & Theory & Simulation & Error & Measured & Error \\
\hline $\mathrm{m}$ & $-7.6542 \times 10^{7}$ & $-7.7598 \times 10^{7}$ & $1.380 \%$ & $-6.6445 \times 10^{7}$ & $13.19 \%$ \\
\hline $\mathrm{b}$ & $7.1155 \times 10^{9}$ & $7.0860 \times 10^{9}$ & $0.408 \%$ & $6.6157 \times 10^{9}$ & $7.024 \%$ \\
\hline $\mathrm{R}^{2}$ & 0.994 & 0.993 & $0.101 \%$ & 0.996 & $0.201 \%$ \\
\hline
\end{tabular}

The measured frequency that was shifted due to parasitic effected the extrapolated linear coefficients; if however a theoretical DCO is designed from $6.66 \mathrm{GHz}$ to $5.59 \mathrm{GHz}$ (shown in Figure 146), then the measured results are almost identical to the theoretical design. See Table 7 for a comparison of the measured and theoretical results of a DCO from $6.66 \mathrm{GHz}$ to $5.59 \mathrm{GHz}$. 


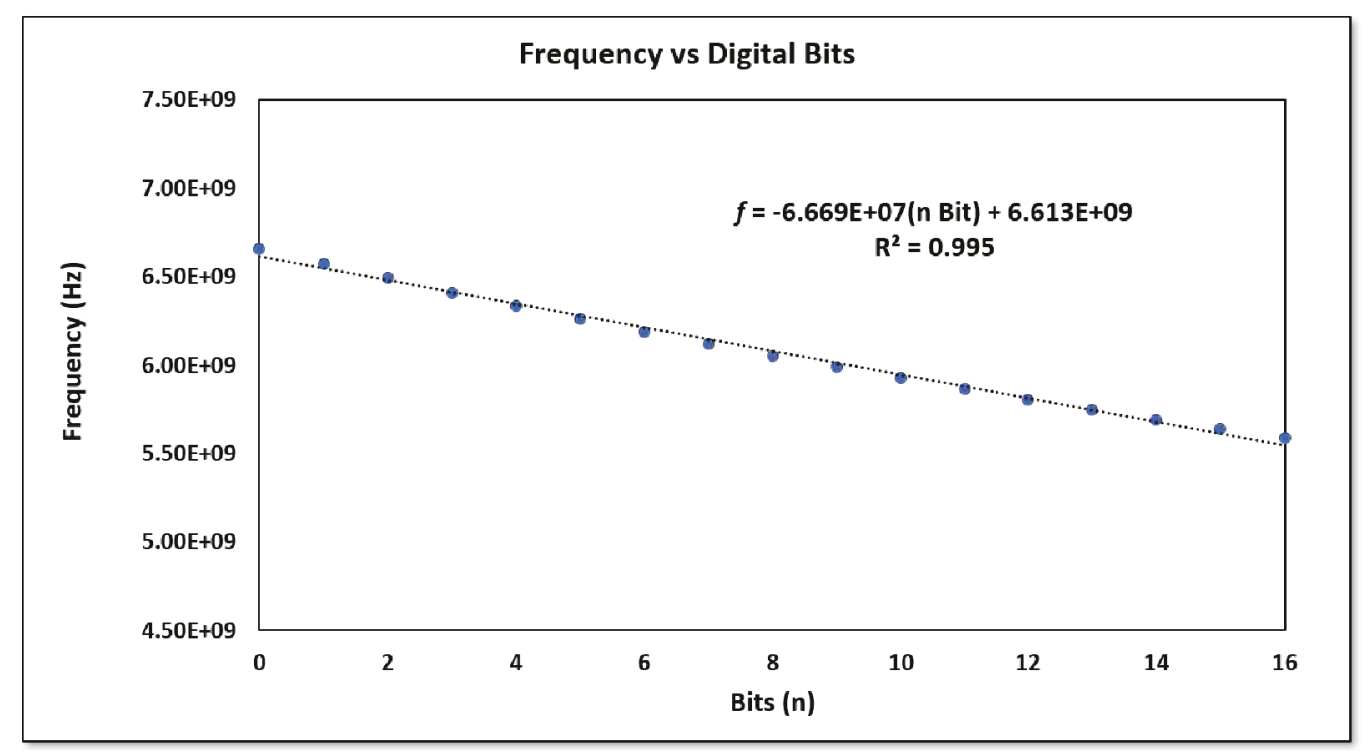

Figure 146: A theoretical DCO from 6.66 GHz to $5.59 \mathrm{GHz}$.

TABLE 7: Comparison Between Lower Frequency Theoretical DCO and Measured DCO.

\begin{tabular}{|c|c|c|c|}
\hline Spec & Theory (new DCO) & Measured & Error \\
\hline $\mathrm{m}$ & $-6.669 \times 10^{7}$ & $-6.6445 \times 10^{7}$ & $0.367 \%$ \\
\hline $\mathrm{b}$ & $6.613 \times 10^{9}$ & $6.6157 \times 10^{9}$ & $0.041 \%$ \\
\hline $\mathrm{R}^{2}$ & 0.995 & 0.996 & $0.101 \%$ \\
\hline
\end{tabular}

The theoretical and measured results are almost identical proving that the design technique/perspective introduced in this thesis is valid and the DCO is extremely linear.

\subsubsection{Linearity analysis and transformation of multiple bank sets}

The slope coefficient $(m)$ of the graph for cycling through Bank sets 1 and 2 was $-8.424 \times 10^{6}$. Overall the cycling had 138 bits (with redundant bank combinations removed). At bit $\sim 125$ the frequency was $5.59 \mathrm{GHz}$. If an equivalent transformation is made to the 16 bit span of Bank set 1, then:

$$
\frac{125 \cdot\left(8.424 \times 10^{6}\right)}{16} \simeq 6.6 \times 10^{7}
$$

which is the same $m$ coefficient from Bank set 1 linear analysis, therefore it can be said that linearity holds true for cycling through Bank set 1 and 2 as it does for Bank set 1 . The measured $\mathrm{R}^{2}$ of cycling through Bank set 1 and 2 was 0.996 which is the same as Bank set 1 . 
Similarly, the slope coefficient $(m)$ of the graph for cycling through Bank sets 1,2 and 3 was $-1.337 \times 10^{6}$. Overall the cycling had 800 bits (with redundant bank combinations removed). At bit $\sim 790$ the frequency was $5.59 \mathrm{GHz}$. If an equivalent transformation is made to the 16 bit span of Bank set 1, then:

$$
\frac{790 \cdot\left(1.337 \times 10^{6}\right)}{16} \simeq 6.6 \times 10^{7}
$$

which is again the same $m$ coefficient from Bank set 1 linear analysis, therefore it can be said that linearity holds true for cycling through Bank set 1, 2, and 3 as it does for Bank set 1 . The measured $\mathrm{R}^{2}$ of cycling through Bank set 1,2 , and 3 was 0.9965 which is approximately the same as Bank set 1; proving that the technique shown in this thesis (for linearity design) is valid even if the initial response of the DCO is not fully monotonic and there is some overlaps of banks (which can possibly be rectified using some pre-processing calibration technique).

\subsection{Phase Noise Comparison}

A method on calculating the theoretical phase noise was given by Eq. 17 in Chapter 3.3. The simulated Q of the DCO core at $6.5 \mathrm{GHz}$ was 5 (see Figure 147. In reality it is closer to 4 since some of the core/bank traces was not extracted due to EMX computational limits.

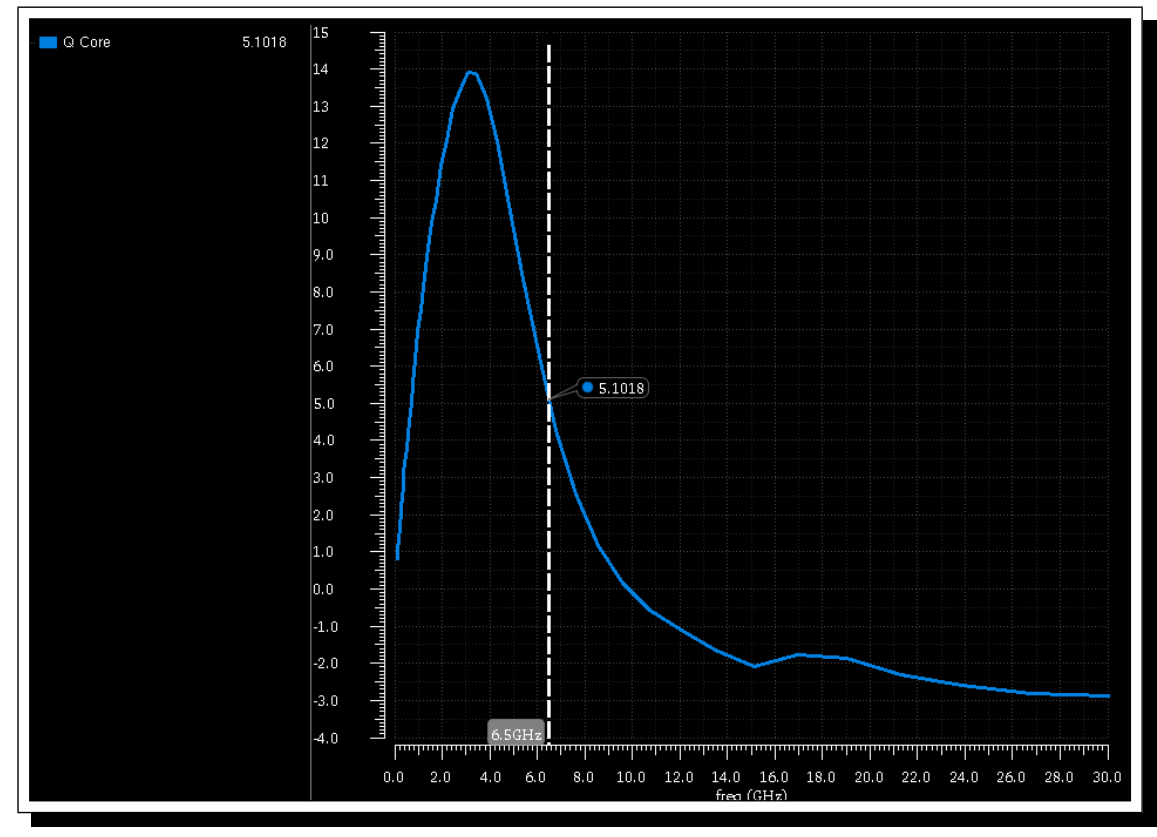

Figure 147: Simulated Q of DCO Core. 
The tank voltage was $2.5 \mathrm{~V}$ (from Figure 64), then:

$$
\begin{gathered}
P=\frac{1}{2} V_{\mathrm{tank}} I=(0.5)(2.5 \mathrm{~V})(14 \mathrm{~mA})=17.5 \mathrm{~mW} \\
P N=10 \cdot \log _{10}\left[\left(\frac{\sqrt{2}(2 \pi 6.5 \mathrm{GHz})}{2(4)(2 \pi 1 \mathrm{MHz})}\right)^{2}\left(\frac{1.67\left(1.38 \times 10^{-} 23 \mathrm{~J} / \mathrm{K}\right)(298 \mathrm{~K})}{2(17.5 \mathrm{~mW})}\right)\right] \simeq-125.87 \mathrm{dBc} / \mathrm{Hz}
\end{gathered}
$$

The simulated phase noise at $6.5 \mathrm{GHz}$ (@ $14 \mathrm{~mA}$ ) was $-125 \mathrm{dBc} / \mathrm{Hz}$ (Figure 74), and the measured phase noise at $6.5 \mathrm{GHz}$ (@ $14 \mathrm{~mA}$ ) was $-115 \mathrm{dBc} / \mathrm{Hz}$. The phase noise comparison is summarized in Table 8 .

TABLE 8: Comparison of Theoretical, Simulated, and Measured PN Results at $6.5 \mathrm{GHz}$ $(I=14 \mathrm{~mA})$.

\begin{tabular}{|c|c|c|c|}
\hline$*$ & Theoretical & Simulation & Measurement \\
\hline $\mathbf{P N}$ & $-126 \mathrm{dBc} / \mathrm{Hz}$ & $-125 \mathrm{dBc} / \mathrm{Hz}$ & $-115 \mathrm{dBc} / \mathrm{Hz}$ \\
\hline
\end{tabular}

The simulated and theoretical values match closely however it is hard to pinpoint what caused the $10 \mathrm{~dB}$ drop in the measured results. Approximately 2-3 dB drop can possibly be attributed to signal degradation from the CML divisions. If the tank Q (in reality) was actually closer to $\sim 1.8$ due to all the metal fills and digital routing traces around the core (not accounted during EMX simulations), then

$P N=10 \cdot \log _{10}\left[\left(\frac{\sqrt{2}(2 \pi 6.5 \mathrm{GHz})}{2(1.8)(2 \pi 1 \mathrm{MHz})}\right)^{2}\left(\frac{1.67\left(1.38 \times 10^{-} 23 \mathrm{~J} / \mathrm{K}\right)(298 \mathrm{~K})}{2(17.5 \mathrm{~mW})}\right)\right] \simeq-119 \mathrm{dBc} / \mathrm{Hz}$

Then you have $2 \mathrm{~dB}$ of CML division performance loss and $2 \mathrm{~dB}$ of digital noise bleed:

$$
P N=-119 \mathrm{dBc} / \mathrm{Hz}+2 \mathrm{~dB}[\mathrm{CML} \text { loss }]+2 \mathrm{~dB}[\text { noise bleed }]=-115 \mathrm{dBc} / \mathrm{Hz}
$$

The above statement can 'potentially' explain why there is noise performance loss. In fact it has been experimentally observed that with a poor digital clock (high jitter), the PN performance got reduced by $10 \mathrm{~dB}$ or more.

Another factor that could have degraded the phase noise was the fact that the short metal traces inside the banks were simulated using ideal wires instead of being fully extracted using EMX (due to computational limits). The parasitic that was not extracted will likely 
decrease the $\mathrm{Q}$ of the bank (since both $\mathrm{R}$ and $\mathrm{C}$ will increase with extraction), and hence degrade the overall PN of the oscillator. Nevertheless the measured FoM at $6.5 \mathrm{GHz}$ (@ 14 $\mathrm{mA}$ ) was $-179 \mathrm{dBc} / \mathrm{Hz}$. While $-179 \mathrm{dBc} / \mathrm{Hz}$ FoM is not at the $-183 \mathrm{dBc} / \mathrm{Hz}$ FoM mark like typical state of the art VCOs, the DCO is still producing decent performance considering the tank core is loaded with lots of banks.

\subsection{FoM Comparison}

The simulated (Figure 78) and measured (Figure 136) curves have similar topology. Of course since the measured phase noise was worse than the simulated phase noise, it is also expected that the measured FoM (Figure 136) would be worse than the simulated FoM (Figure 78).

\subsection{Conclusion}

This chapter analyzed the theoretical, simulated, and measured results of the DCO. The measured frequency response of the DCO dropped by $480 \mathrm{MHz}$ from simulated results for the upper frequency and $290 \mathrm{MHz}$ for the lower frequency. This was probably because of parasitic capacitance around the core of the oscillator due to kit metal fill density requirements. The phase noise of the measured DCO was almost $10 \mathrm{~dB}$ lower than the simulated and theoretical values leading to believe that the $\mathrm{Q}$ of the core was worse than simulated, that there was digital bleeding and that there was quite a loss from CML performance $(2-3 \mathrm{~dB})$. Nevertheless the measured FoM at $6.5 \mathrm{GHz}$ (@ $14 \mathrm{~mA}$ ) was -179 $\mathrm{dBc} / \mathrm{Hz}$ which was still a decent performance. 


\section{CHAPTER 11: EXTRA WORK}

This chapter will discuss any extra work that has been done as either part of the thesis project or work related to the thesis project.

\subsection{Frequency Divider to Route DCO Signal to DLF}

The digitally controlled oscillator was part of an all-digital phase-locked loop. Additional work was done to route the signal from the DCO and provide a feasible clock for the TDC and DLF.

A divider that divides by $2,3,4 \ldots$ all the way to 31 was designed, and then laid out in Layout XL to drive the clock signal of the loop filter. The divider took the signal from the first division of the CML. Figure 148 shows the divider chain connection from the DCO. See Figure 149 for the layout of the divider. See Figure 150 for a photographic table outlining the control bits that was used to control of the divisions of the divider. See reference [47] for a generalized schematic of the divider.

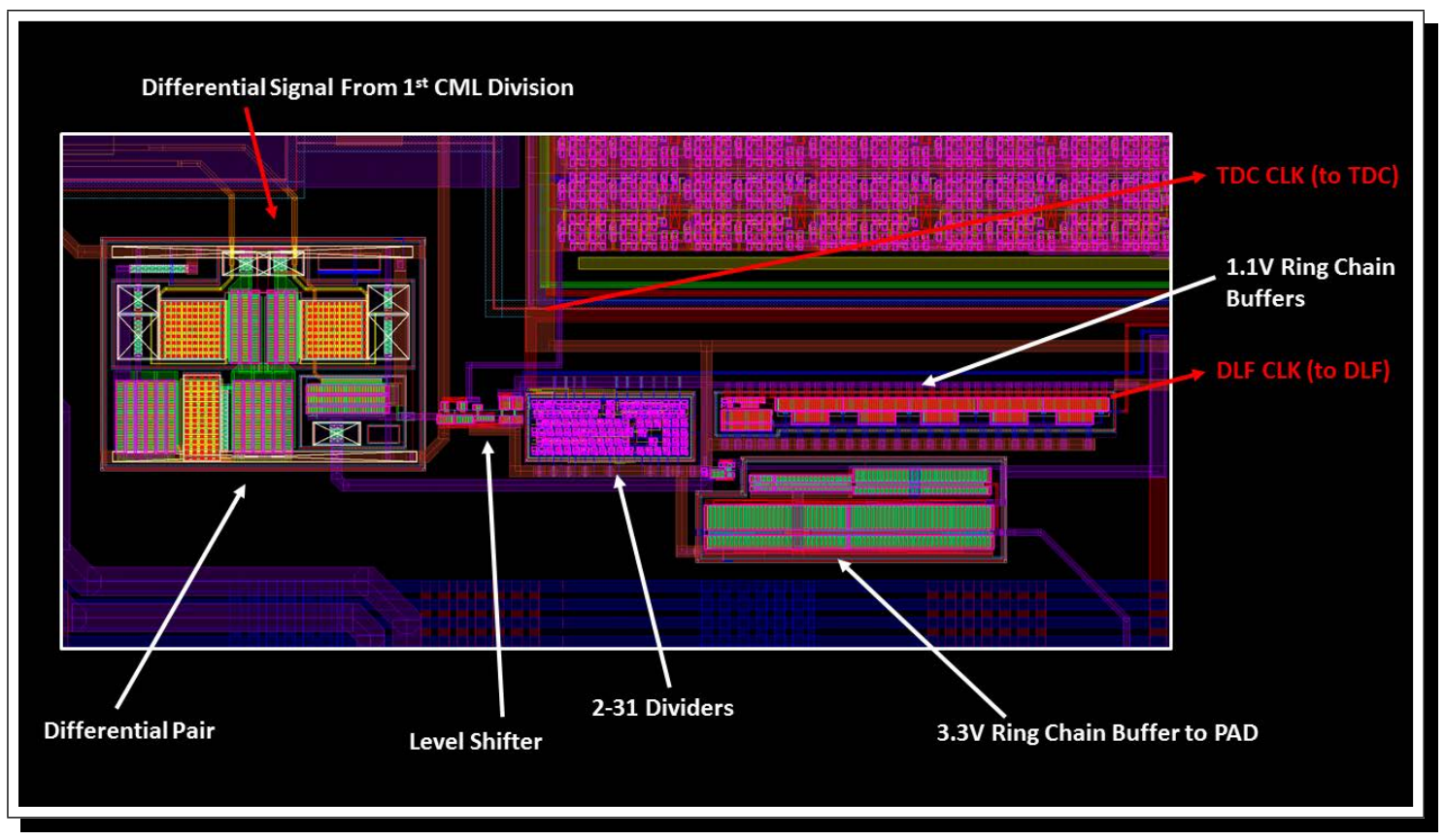

Figure 148: Signal propagating from CML to $(\div 2-39)$ dividers. 


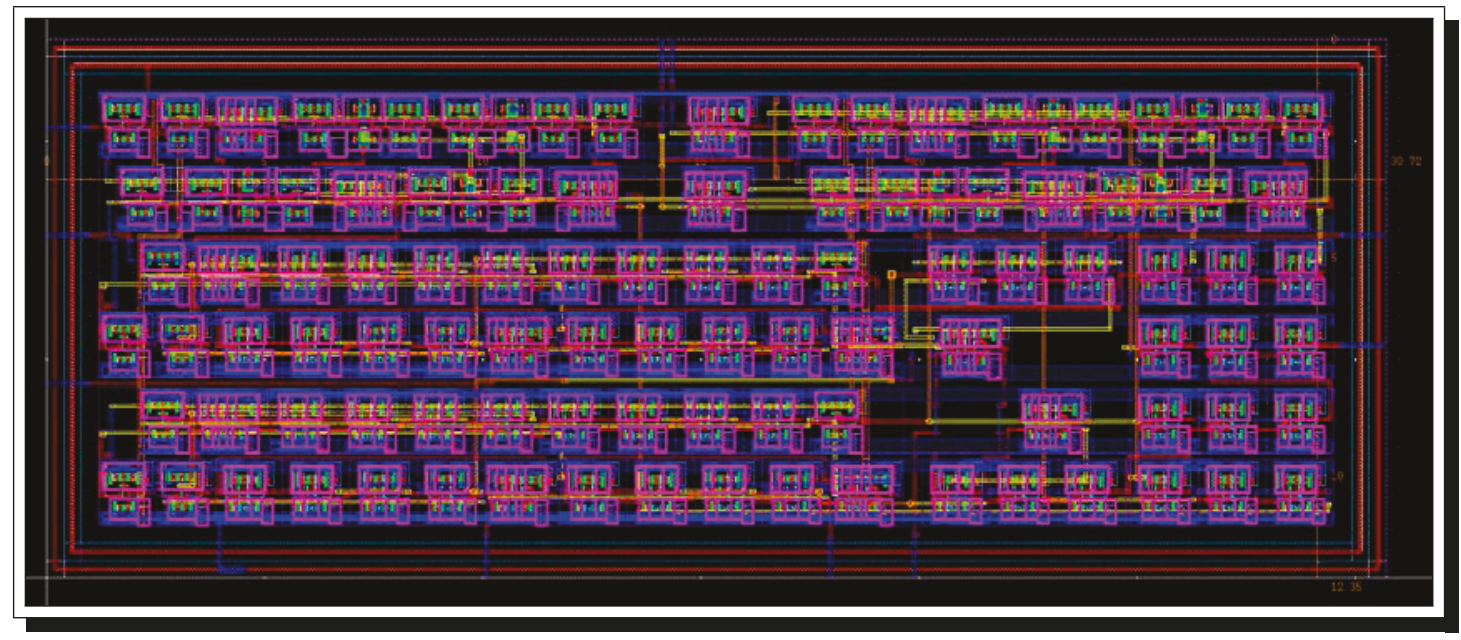

Figure 149: Layout of the divider block.

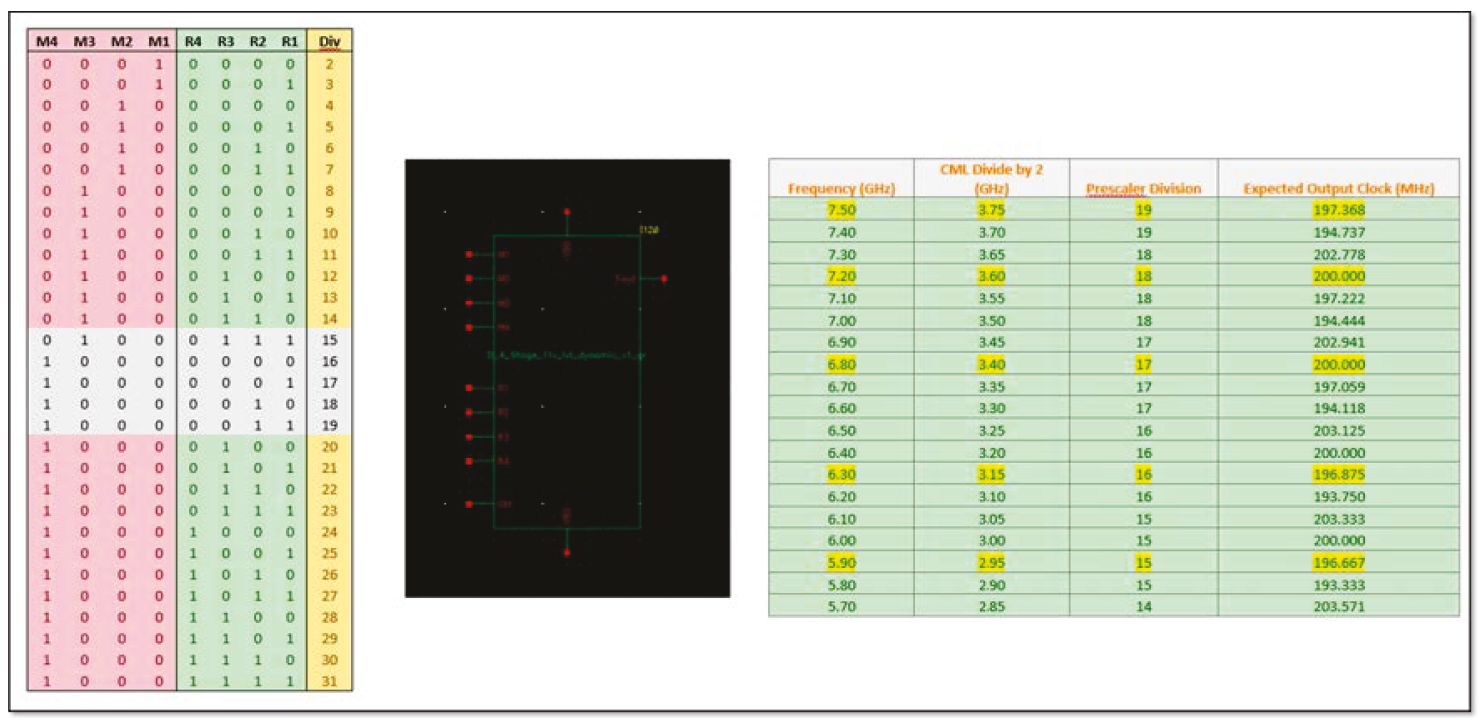

Figure 150: Divider control signals and expected output frequencies.

See Figure 151 to Figure 154 for the transient simulation results of the divided frequency. 
Oscillator Frequency: $7.5 \mathrm{GHz}$

CML Divide by 2 Frequency: $3.75 \mathrm{GHz}$

Pre-scaler Division by 19

Expected Frequency: $197.368 \mathrm{MHz}$

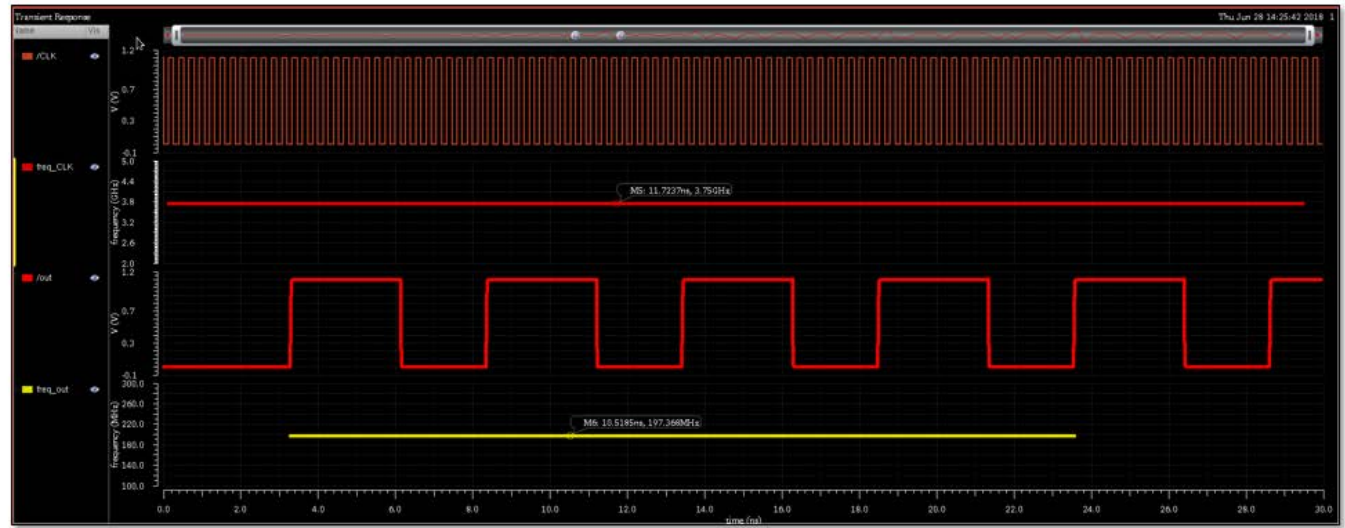

RCL Simulation Results:

Output Frequency: 197.368 MHz

Figure 151: Division by 19 .

Oscillator Frequency: $7.2 \mathrm{GHz}$

CML Divide by 2 Frequency: $3.6 \mathrm{GHz}$

Pre-scaler Division by 18

Expected Frequency: $200.000 \mathrm{MHz}$

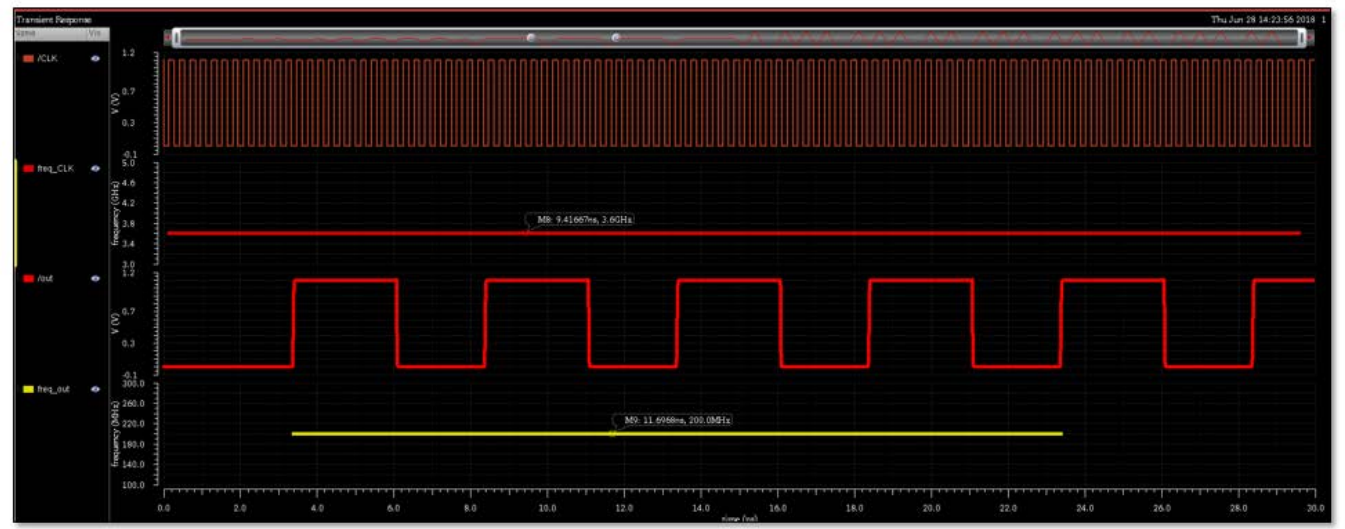

RLC Simulation Results:

Output Frequency: $200.0 \mathrm{MHz}$

Figure 152: Division by 18. 
Oscillator Frequency: $6.8 \mathrm{GHz}$

CML Divide by 2 Frequency: $3.4 \mathrm{GHz}$

Pre-scaler Division by 17

Expected Frequency: $200.000 \mathrm{MHz}$

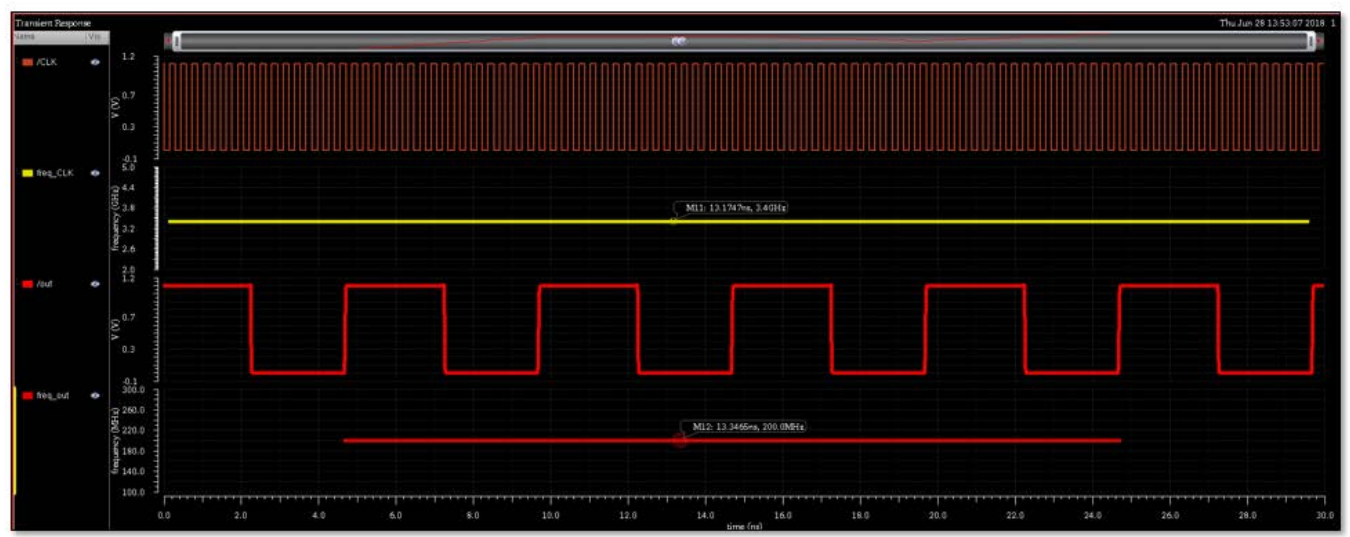

RLC Simulation Results:

Output Frequency: $200.0 \mathrm{MHz}$

Figure 153: Division by 17.

Oscillator Frequency: $6.3 \mathrm{GHz}$

CML Divide by 2 Frequency: $3.15 \mathrm{GHz}$

Pre-scaler Division by 16

Expected Frequency: $196.875 \mathrm{MHz}$

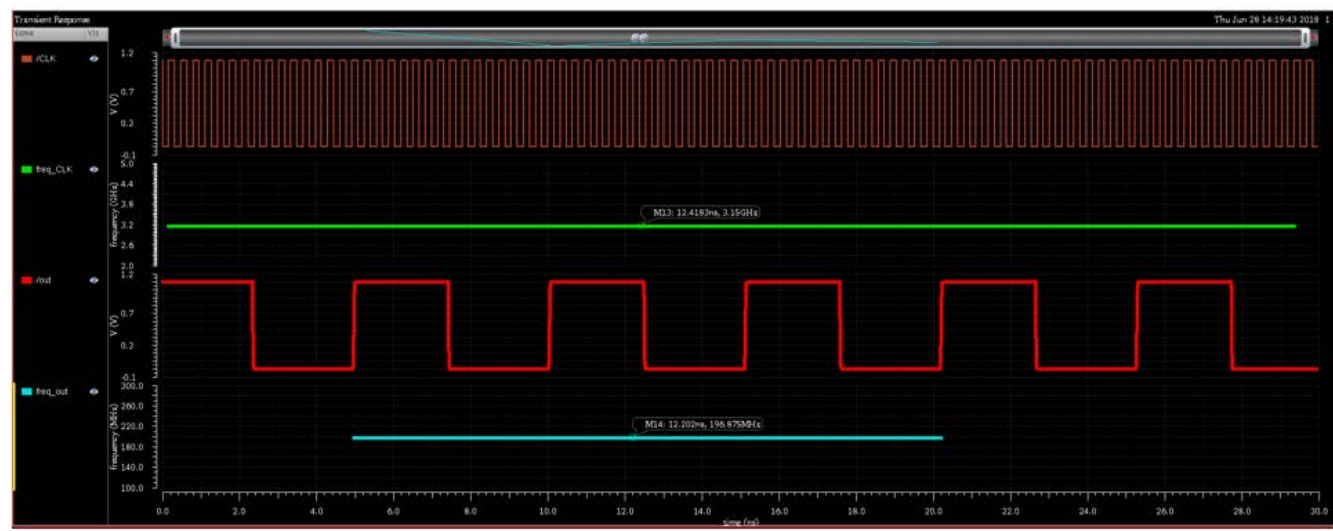

RLC Simulation Results:

Output Frequency: $196.875 \mathrm{MHz}$

Figure 154: Division by 16 .

Currently the divider chain is being debugged at the time of writing this thesis.

\subsection{Second Digital Controlled Oscillator to Verify Simulator Integrity}

Since the main digitally controlled oscillator worked almost as intended, not much work was spent on verifying the second DCO. The oscillator was built mainly to assess whether 
EMX or RLC (xL) Calibre simulator [46] (or both) was correct. An FFT measurement (from spectrum analyzer) of the frequency response of the oscillator when all banks are off is shown in Figure 155.

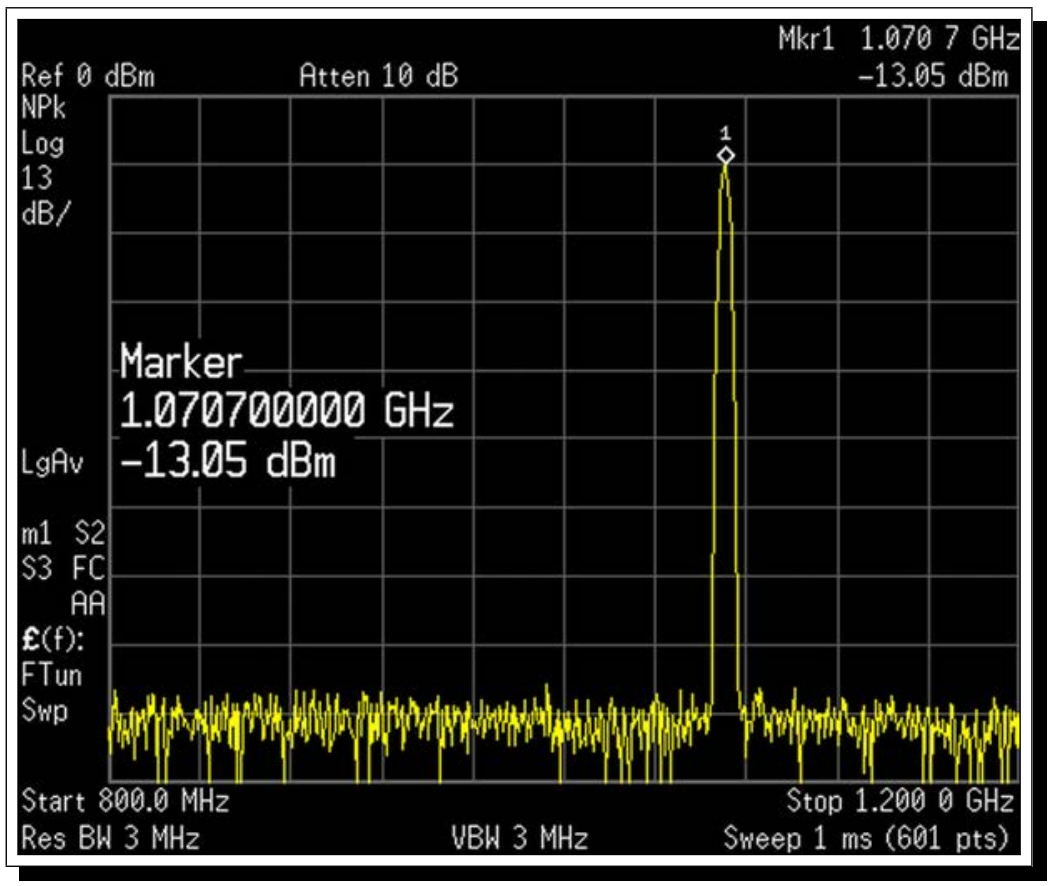

Figure 155: Frequency response of DCO 2 when all banks are off. Sweep was $1 \mathrm{~ms}$ and bandwidth was $3 \mathrm{MHz}$.

The measured frequency was $1.0707 \mathrm{GHz}$, this means the frequency of the oscillator was $17.1312 \mathrm{GHz}$ (the frequency was measured at the output of 4 CML divisions - $\div$ by 16). This suggests that EMX was most likely the correct simulator engine and superior to RLC.

\subsection{DCO in 65nm Kit to Verify Theoretical Results}

A second stripped down digital controlled oscillator in $65 \mathrm{~nm}$ kit was designed and laid out to verify the integrity of the theory proposed in this thesis. The layout of the oscillator is shown in Figure 156. 


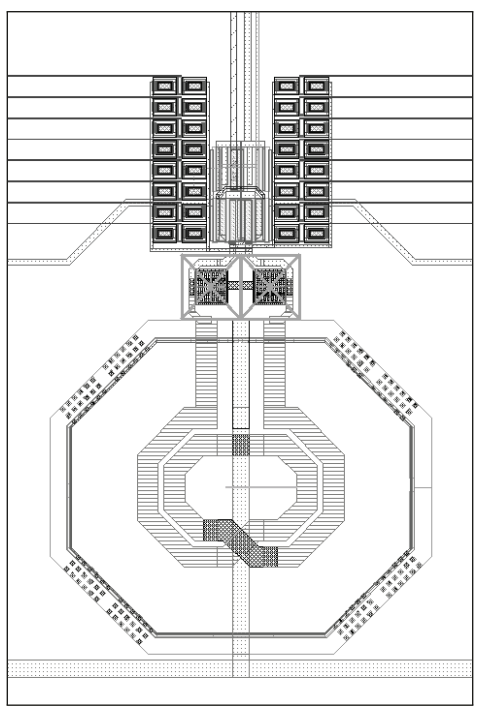

Figure 156: Layout of DCO in $65 \mathrm{~nm}$ (TSMC kit).

The simulated frequency response of the $65 \mathrm{~nm}$ kit DCO is shown in Figure 157 . The simulated results of the oscillator in comparison to theory is summarized in Table 9.

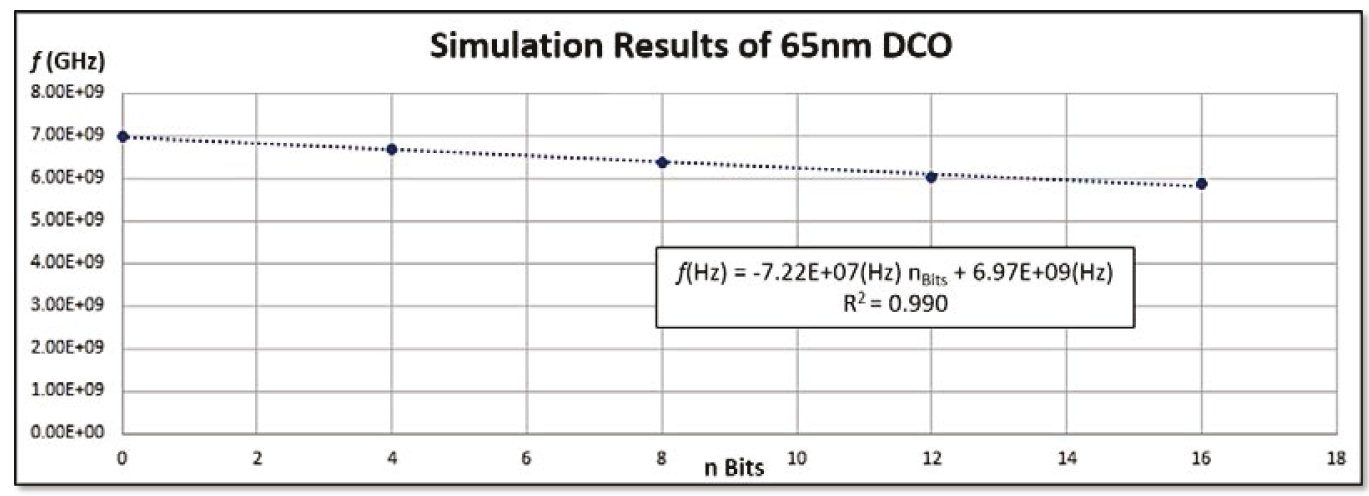

Figure 157: Frequency simulation results of $65 \mathrm{~nm}$ DCO.

TABLE 9: Comparison of Theoretical vs Simulated Results of 65nm Oscillator

\begin{tabular}{|c|c|c|c|}
\hline Spec & Prediction & 65 nm RLC & Error \\
\hline $\mathbf{m}$ & $-7.64 \times 10^{7}$ & $-7.22 \times 10^{7}$ & $5.50 \%$ \\
\hline $\mathbf{b}$ & $7.11 \times 10^{9}$ & $6.97 \times 10^{9}$ & $0.20 \%$ \\
\hline $\mathbf{R}^{\mathbf{2}}$ & 0.995 & 0.990 & $0.50 \%$ \\
\hline
\end{tabular}

The (simulated) $65 \mathrm{~nm}$ DCO linear characteristics was almost the same as the theoretical DCO proposed in Chapter 4; suggesting the linear theorem and design proposed in this thesis is valid. 


\subsubsection{Tuning range of $65 \mathrm{~nm}$ oscillator}

The tuning range of the simulated $65 \mathrm{~nm}$ DCO was:

$$
\text { Tunning Range }(\mathrm{TR}) \%=100 \cdot\left[\frac{2(6.977 \mathrm{GHz})-2(5.883 \mathrm{GHz})}{6.977 \mathrm{GHz}+5.883 \mathrm{GHz}}\right] \approx 17.01 \%
$$

The $65 \mathrm{~nm}$ simulated DCO had almost the same tuning range as the theoretical DCO in Chapter 4 and the $40 \mathrm{~nm}$ simulated DCO in Chapter 7.

\subsection{Low Frequency Oscillator to Verify Theoretical Results}

A low frequency oscillator (in $\mathrm{kHz}$ range) was also designed to verify the integrity of the linearity theory. The schematic of the oscillator is shown in Figure 158 and the frequency simulation of the oscillator is shown in Figure 159. A transient simulation is shown in 160. A comparison of the linearity of the DCO with theoretical values is summarized in Table 10.

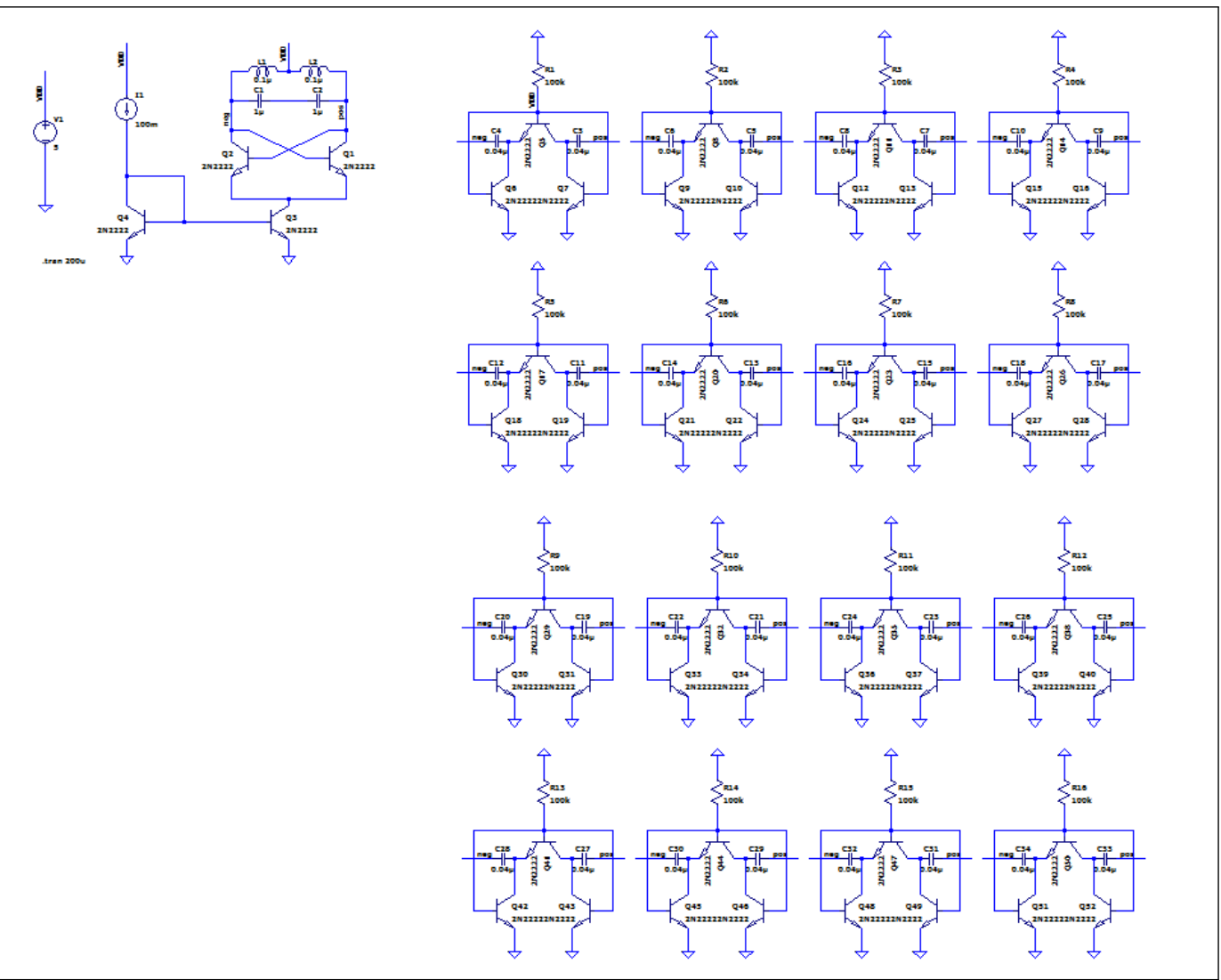

Figure 158: Schematic design of low frequency DCO. 


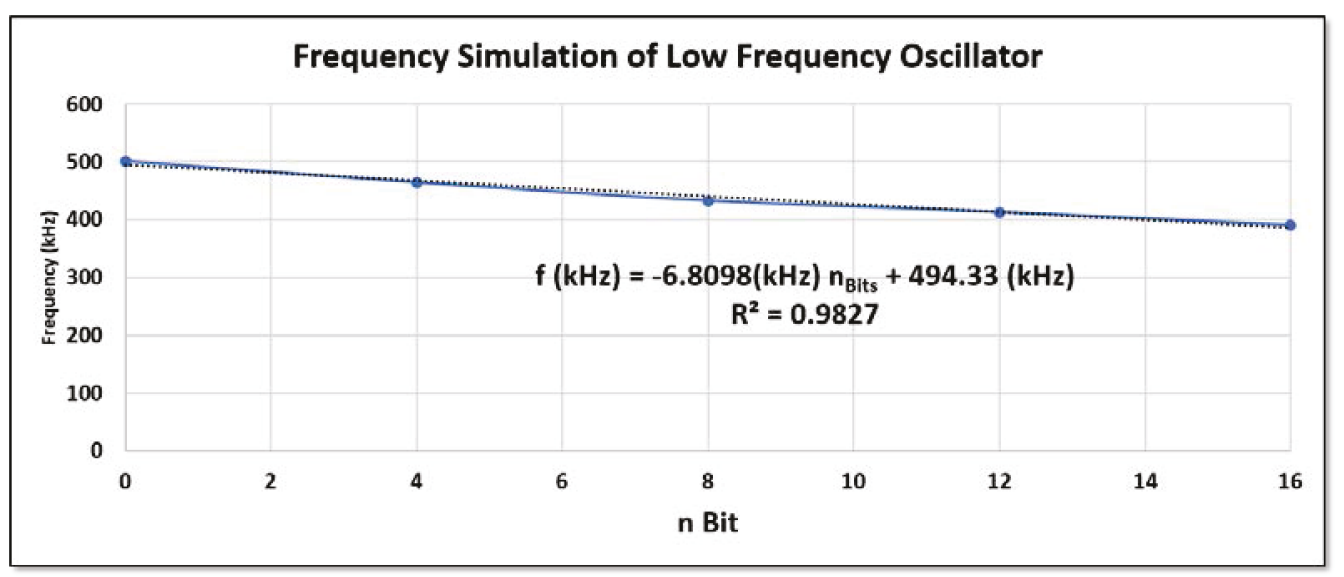

Figure 159: Frequency simulation of a low frequency oscillator.

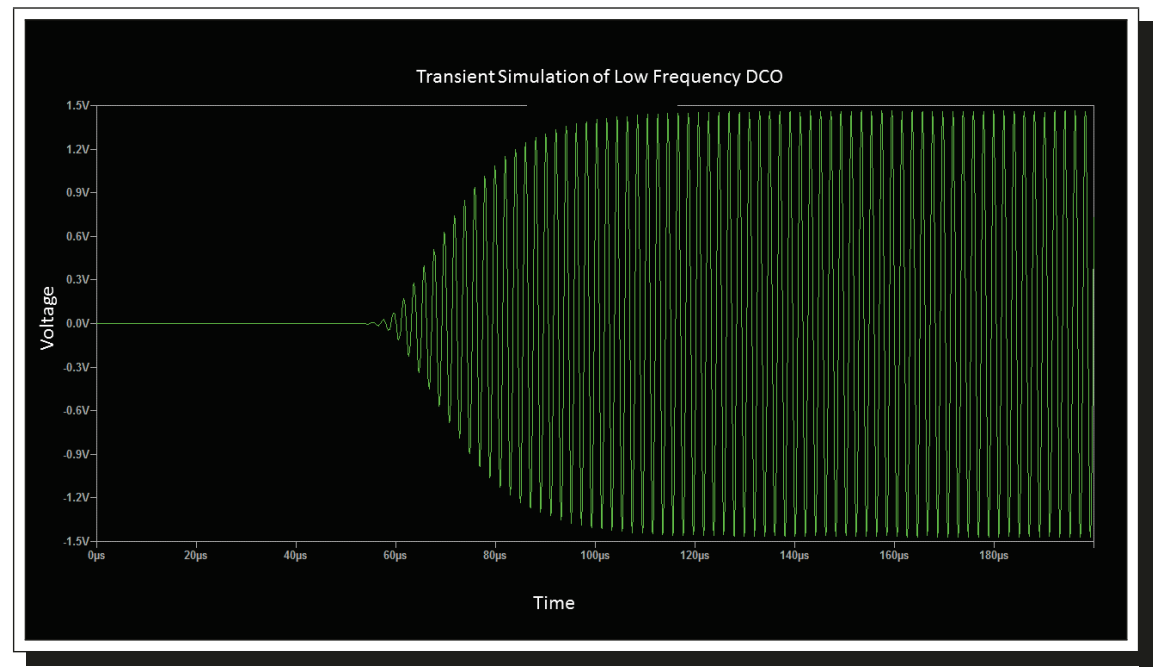

Figure 160: Transient response of the low frequency DCO.

TABLE 10: Comparison of Theoretical vs Simulated Results of Low Frequency Oscillator.

\begin{tabular}{|c|c|c|c|}
\hline Spec & Prediction & Simulation & Error \\
\hline $\mathbf{m}$ & $-6.841 \times 10^{3}$ & $-6.8098 \times 10^{3}$ & $0.456 \%$ \\
\hline $\mathbf{b}$ & $4.936 \times 10^{5}$ & $4.943 \times 10^{5}$ & $0.142 \%$ \\
\hline $\mathbf{R}^{\mathbf{2}}$ & 0.9896 & 0.9827 & $0.697 \%$ \\
\hline
\end{tabular}

\subsubsection{Tuning range of the low frequency DCO}

The simulated tuning range of the low frequency oscillator was: 


$$
\text { Tunning Range }(\mathrm{TR}) \%=100 \cdot\left[\frac{2(500.66 \mathrm{kHz})-2(390.09 \mathrm{kHz})}{500.66 \mathrm{kHz}+390.06 \mathrm{kHz}}\right] \approx 24.83 \%
$$

The theoretical tuning range was:

$$
\text { Tunning Range }(\mathrm{TR}) \%=100 \cdot\left[\frac{2(501 \mathrm{kHz})-2(390 \mathrm{kHz})}{501 \mathrm{kHz}+390 \mathrm{kHz}}\right] \approx 24.92 \%
$$

\subsection{Conclusion}

This chapter discussed any extra work that has been done either as part of the thesis project or any work related to the thesis project. Some extra work including making a frequency divider that can divide from 2 to 31 , and designing a DCO in $65 \mathrm{~nm}$ kit to verify the integrity of the linearity theorem proposed in Chapter 4. The results of the $65 \mathrm{~nm}$ DCO concurred with the theoretical results. A second DCO with low frequency $(\mathrm{kHz}$ range) was designed to verify the theorem as well and it also concurred with the theory. 


\section{CHAPTER 12: FUTURE WORK}

Future work includes design changes such that the frequency is adjusted to hit the $7 \mathrm{GHz}$ to $6 \mathrm{GHz}$ mark instead of the current $6.5 \mathrm{GHz}$ to $5.5 \mathrm{GHz}$. This can be easily done by altering the inductor value as a first attempt.

A design iteration of the DCO is needed to decrease the settling time of the output frequency; and it is presumed that this can be achieved by adding buffers in between the digital bits that control the oscillator and the digital circuits.

Future work also includes getting the full ADPLL loop to work. Currently the dividers clocking the SPI-DLF and the signal leading into the TDC is being debugged and investigated; with the culprit that is causing the issue being highly presaged as the level shifter and/or differential pair (in the pathway leading from the DCO to the TDC).

It was concluded that digital noise bleeding might have been a culprit leading to the degradation of phase noise performance. More investigation is needed and better work needs to be done in isolating the digital circuits from the core of the DCO by using bigger and thicker wells.

Further investigation needs to be done regarding Bank set 5 to Bank set 11 as it did not work as intended. It is unsure at the time of writing the thesis as to why the issue is. It is hypothesised it can be due to either measuring equipment resolution limits, DCO resolution limits due to parasitic, or because the bank sets were on the source side of the oscillator. All of these possibilities confounds the issue and further studies need to be done to resolve the problem.

The CML still needs to be fully characterized, simulations showed that it caused $2 \mathrm{~dB}$ of performance loss, and it is presumed (in reality) that it is probably closer to $3 \mathrm{~dB}$ of performance loss, however it needs to be quantified by measurements.

An enormous amount of work that is still left to do, and it is left possibly for a higher post-graduate (Ph. D) degree, is to design an automated calibration algorithm that removes duplicate bank combinations (that will not only fully linearize the DCO but also make the 
DCO monotonic). An example of how the calibration scheme might be implemented is shown in Figure 161, and a possible algorithm that might work for the calibration scheme is shown in Figure 162

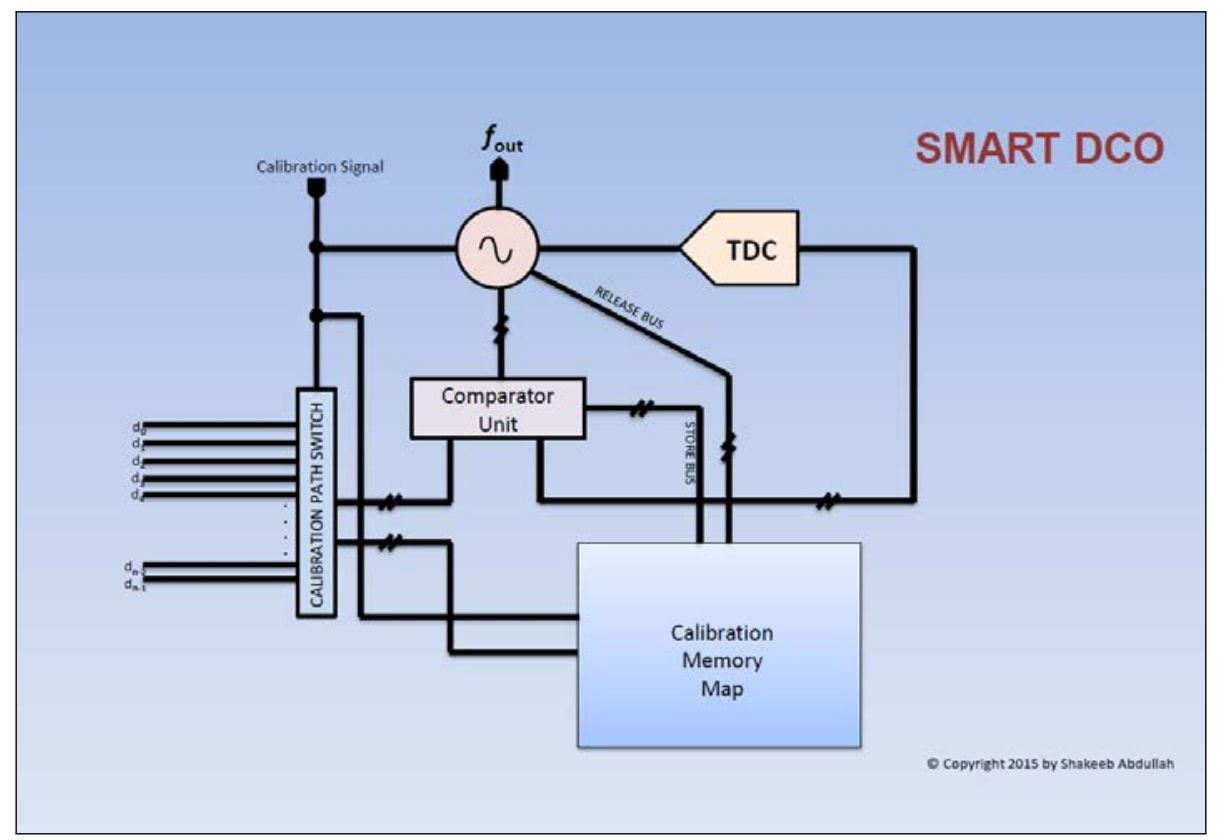

Figure 161: Linearization calibration scheme.

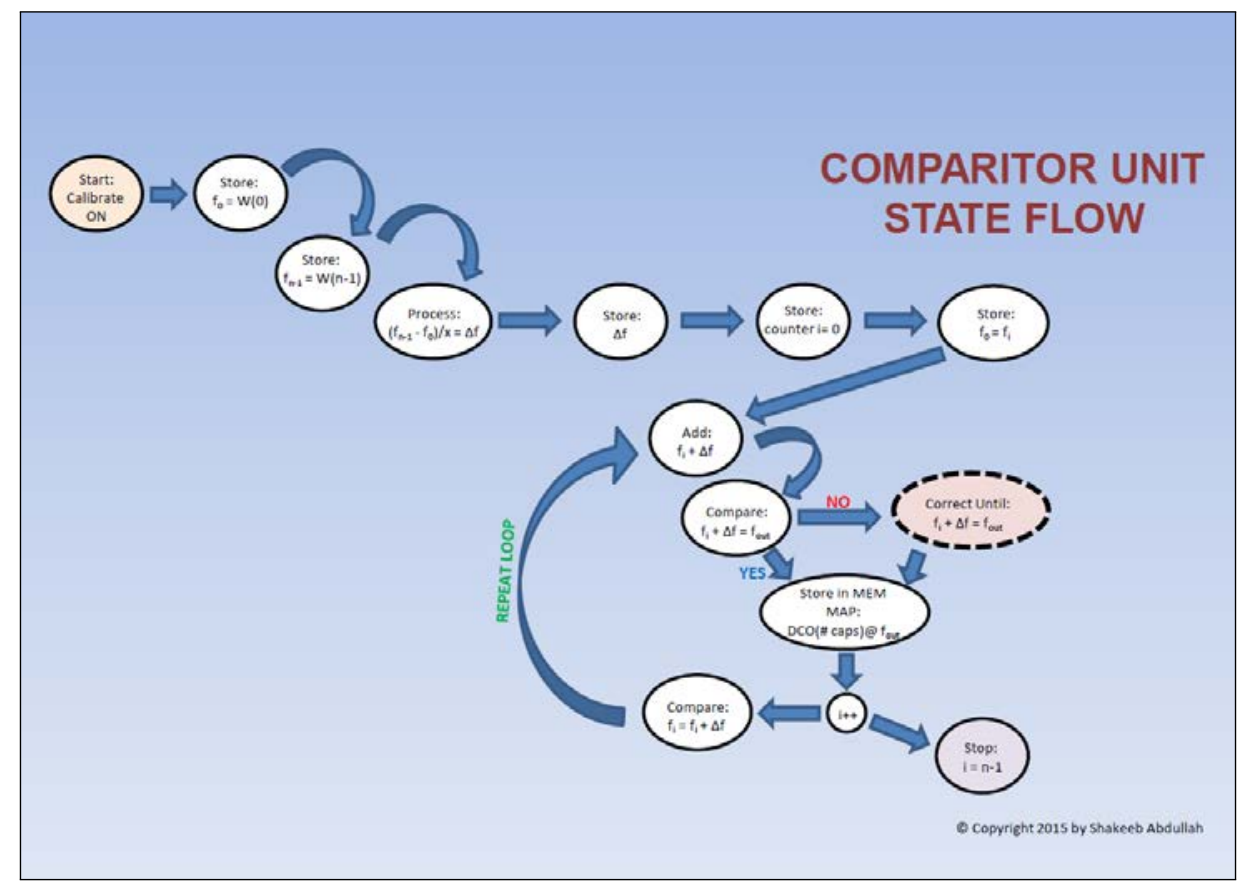

Figure 162: Sample model of the linearization algorithm. 
A sample execution of the algorithm is shown in Figure 186.

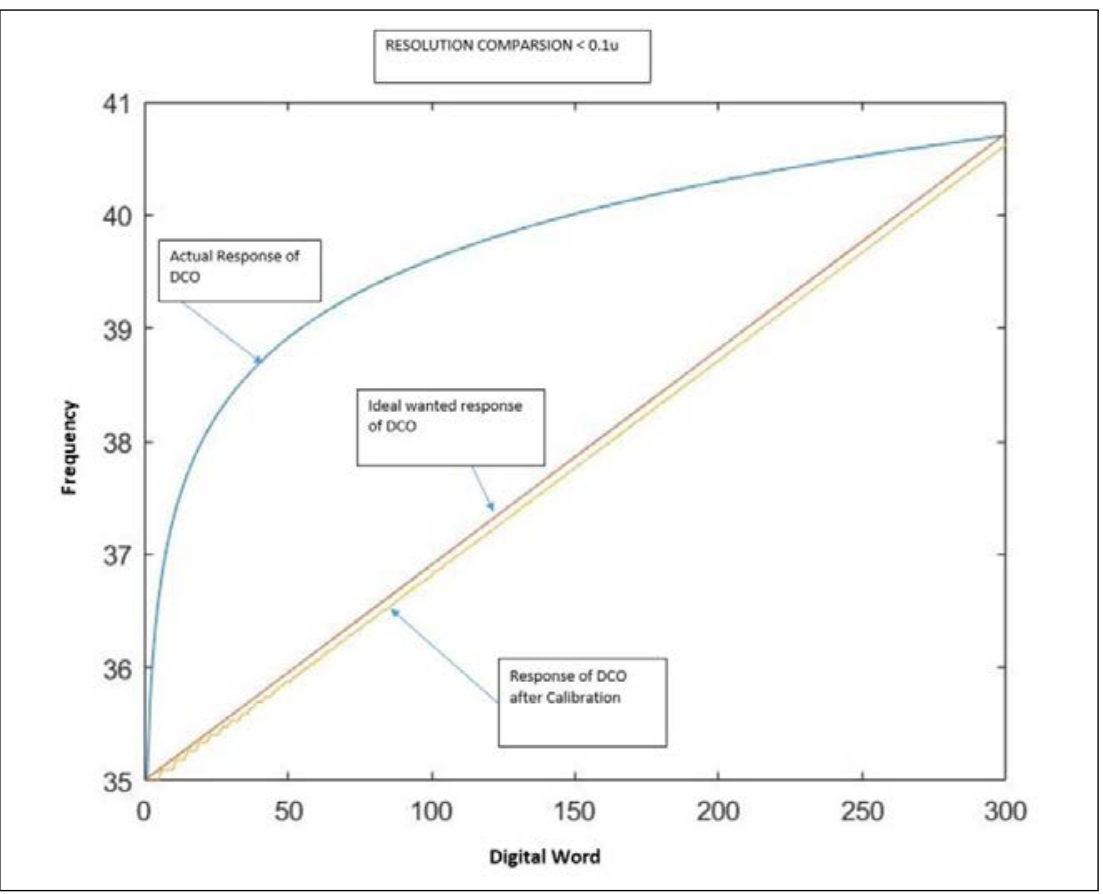

Figure 163: Example simulation of the linearization algorithm.

Overall there is still plenty of work left to do regarding the DCO before it can be used as a daily driver for products; however as a first step the DCO experiment was a great success.

\subsection{Conclusion}

This chapter discussed any future work that is still left to be done regarding the DCO. Future work includes decreasing the frequency settling time of the DCO once a bank is switched. Other future work included characterizing the CMLs and debugging the signals leading up to the TDC from the DCO. Future work also included getting a full ADPLL loop to work and investigating why bank sets 5 to 11 did not work as intended. 


\section{CHAPTER 13: THESIS SUMMARY}

This thesis presented a new outlook or perspective on how to design an extremely linear digitally controlled oscillators using a new concept known as linearity field maps. The thesis first analyzed the theory regarding linearity and tuning range. It then proposed a methodological way on how to design a theoretical linear DCO. It then showed how to implement the theoretical DCO in practice by using a cascading pyramid scheme of banks within banks to achieve the linearity. The contributions of the thesis were:

- Introducing a new concept known as linearity field maps.

- Using $R^{2}$ as a tool for assessing linearity.

- Showing how to theoretically design a linear LC DCO using the field maps.

- And then creating a physical LC DCO using a cascading pyramid scheme of equal banks within equal banks to achieve the linearity.

The theoretical and simulated DCOs were congruent with each other, in fact the linear

coefficient errors ( $\mathrm{m}, \mathrm{b}$, and $\mathrm{R}^{2}$ ) were less than $1 \%$; however the measured DCO's frequency response was slightly lower than the simulated results. This could have been because of additional parasitic around the DCO core due to metal fills (required for chip tape-out).

The simulated and theoretical frequency response of the DCO was from $7.1 \mathrm{GHz}-5.7$ $\mathrm{GHz}$ whereas the measured frequency response of the DCO was from 6.6 Ghz to $5.5 \mathrm{GHz}$. This caused all the linear coefficients of the measured DCO to be mismatched with the initial theoretical values. However when a new theoretical DCO that ranges with the same frequency span as the measured DCO is compared, then the coefficient errors were less than $1 \%$, proving that the design technique presented in this thesis was valid. The $\mathrm{R}^{2}$ value of the measured DCO was 0.996 which was extremely linear.

The theoretical phase noise and simulated phase noise concurred with each other $(-125$ $\mathrm{dBc} / \mathrm{Hz}$ ) however the measured phase noise was almost $10 \mathrm{~dB}$ lower. About 2-3 dB loss could have been attributed due to poor CML performance. Some digital noise bleeding could have also played a role in reducing the oscillators performance, nevertheless FoM was still reasonable and respectable with FoM better than or equal to $-175 \mathrm{dBc} / \mathrm{Hz}$ throughout the entire span of the DCO (at it's highest frequencies when all banks are off and at its 
lowest frequency when all banks are on).

The measured Bank set 1 had a frequency step size of $66.4 \mathrm{MHz}$. The measured Bank set 2 upper limit frequency step size was $9.91 \mathrm{MHz}$ and lower limit frequency step size was 6.20 MHz. The measured Bank set 3 had upper limit frequency step size of $2.09 \mathrm{MHz}$ and lower limit frequency step size $1.29 \mathrm{MHz}$. The measured Bank set 4 had upper limit frequency step size of $469 \mathrm{kHz}$ and lower limit frequency step size of $267 \mathrm{kHz}$.

Extra work was also done to verify the theorem proposed in this thesis. A DCO designed in lower $\mathrm{kHz}$ frequency range concurred with the theory proposed in this thesis as did a second 7-6 GHz DCO designed in 65nm kit.

There is still plenty of work left to improve the DCO. Future work includes fixing the settling time of the DCO when a bank is switched as it currently takes too long to settle. Further investigation needs to be done regarding Bank set 5 to Bank set 11 as to why it did not function as intended.

Nevertheless, overall the thesis experiment was a success in showing how to design a linear digitally controlled oscillator, the DCO in the thesis worked even though frequency was shifted, and the point of the thesis was made with regards to linearity.

\subsection{Conclusion}

The reason for this report was to satisfy the requirements for MASc graduate degree at Carleton University and the purpose of this thesis was to present a new perspective on how to design an extremely linear digitally controlled oscillator. This was achieved by using equal bank steps in each bank sets. The thesis first analyzed the theory regarding linearity and then manifested it in a physical DCO. The DCO's frequency span was shifted from the simulated results (possibly due to parasitic arising from metal fills and not fully extracting the banks during simulations), nevertheless the DCO had a decent FoM better than $-175 \mathrm{dBc} / \mathrm{Hz}$ through its frequency span and for different power levels. Overall the thesis experiment was a success in implementing a linear DCO, the DCO in the thesis worked, and the point of the thesis was made. The thesis showed how to design a linear digitally controlled oscillator. 


\section{REFERENCES}

[1] J. W. M. Rogers and C. Plett, Radio Frequency Integrated Circuit Design. Norwood: Artech House, 2010.

[2] J. Wight, Lecture Notes for ELEC 5606, Phase-Locked Loops and Receiver Synchronizers. Carleton University at Ottawa, Fall semester, 2016.

[3] A. Blanchard, Phase-Locked Loops: Application to Coherent Receiver Design.. New York, NY, USA: Willey, 1976.

[4] A. Tomar, R. K. Pokharel, H. Kanaya and K. Yoshida, "Design of Monotonic Digitally Controlled Oscillator (DCO) for Wide Tuning Range, KIET International Journal of Communications and Electronics, vol. 1, no. 3, pp. 28-33, Dec. 2013.

[5] J. Gorji and M. B. Ghaznavi-Ghoushchi, "A Process-Independent and Highly Linear DCO for Crowded Heterogeneous IoT Devices in 65-nm CMOS," in IEEE Transactions on Very Large Scale Integration (VLSI) Systems, vol. PP, no. 99, pp. 1-11.

[6] T. S. Rathore, "Synthesis and classification of LC oscillators," 2017 2nd International Conference on Communication Systems, Computing and IT Applications (CSCITA), Mumbai, India, 2017, pp. 28-32.

[7] Tseng-Chuan Wang, "Design of tuning bandwidth and linearity for VCO by mathematical solution and computer method," RAWCON 2000. 2000 IEEE Radio and Wireless Conference (Cat. No.00EX404), Denver, CO, 2000, pp. 155-158.

[8] Jing Yongqi, He Guoyu and Xu Ding, "A new realizable technique to improve the VCO linearity," 6th International Symposium on Antennas, Propagation and EM Theory, 2003. Proceedings. 2003, Beijing, China, 2003, pp. 396-399.

[9] S. Kurachi, T. Yoshimasu, N. Itoh and K. Yonemura, "5-GHz Band Highly Linear VCO IC with a Novel Resonant Circuit," 2007 Topical Meeting on Silicon Monolithic Integrated Circuits in RF Systems, Long Beach, CA, 2007, pp. 285-288.

[10] L. Grigorescu and I. Diaconescu, "Theoretical and experimental research on the current-mode RC oscillators," 2016 8th International Conference on Electronics, Computers and Artificial Intelligence (ECAI), Ploiesti, 2016, pp. 1-6.

[11] F. Colodro and A. Torralba, "Linearity Enhancement of VCO-Based Quantizers for SD Modulators by Means of a Tracking Loop," in IEEE Transactions on Circuits and Systems II: Express Briefs, vol. 61, no. 6, pp. 383-387, June 2014.

[12] H. Venkatram, R. Inti and Un-Ku Moon, "Least Mean Square calibration method for VCO non-linearity," 2010 International Conference on Microelectronics, Cairo, 2010, pp. 1-4.

[13] Yun Chiu, C. W. Tsang, B. Nikolic and P. R. Gray, "Least mean square adaptive digital background calibration of pipelined analog-to-digital converters," in IEEE Transactions on Circuits and Systems I: Regular Papers, vol. 51, no. 1, pp. 38-46, Jan. 2004. 
[14] Nguyen Phuong Thi Le, K. T. Low and Libin Yao, "High linearity voltage-controlled oscillator," 2009 IEEE 8th International Conference on ASIC, Changsha, Hunan, 2009, pp. 363-366.

[15] B. Hanafi and E. Hegazi, "A Technique for Truly Linear LC VCO Tuning, a Proof of Concept," 2007 Internatonal Conference on Microelectronics, Cairo, 2007, pp. 93-96.

[16] S. Kurachi, T. Yoshimasu, N. Itoh and K. Yonemura, "5-GHz Band Highly Linear VCO IC with a Novel Resonant Circuit," 2007 Topical Meeting on Silicon Monolithic Integrated Circuits in RF Systems, Long Beach, CA, 2007, pp. 285-288.

[17] U. Wismar, D. Wisland and P. Andreani, "Linearity of bulk-controlled inverter ring VCO in weak and strong inversion," 2005 NORCHIP, Oulu, Finland, 2005, pp. 145-148.

[18] J. A. McNeill, "Interpolating ring VCO with V-to-f linearity compensation," in Electronics Letters, vol. 30, no. 24, pp. 2003-2004, 24 Nov. 1994.

[19] Jing Yongqi, He Guoyu and Xu Ding, "A new realizable technique to improve the VCO linearity," 6th International Symposium on Antennas, Propagation and EM Theory, 2003. Proceedings. 2003, Beijing, China, 2003, pp. 396-399.

[20] L. Liangbing and T. Yaqing, "A new realizable structure to improve VCO linearity," 2007 8th International Conference on Electronic Measurement and Instruments, Xi'an, 2007, pp. 4-625-4-629.

[21] E. Ayranci, K. Christensen and P. Andreani, "Enhancement of VCO Linearity and Phase Noise by Implementing Frequency Locked Loop," EUROCON 2007 - The International Conference on "Computer as a Tool", Warsaw, 2007, pp. 2593-2599.

[22] K. H. Koo, S. W. Yoon and C. W. Kim, "A 34\% tuning range CMOS VCO with linear frequency tuning," 2009 IEEE Radio and Wireless Symposium, San Diego, CA, 2009, pp. 288-291.

[23] S. Jain, F. Ellinger and N. T. Tchamov, "Fast sampling DCO for $2.4 \mathrm{GHz}$ wake-up receiver," in Electronics Letters, vol. 52, no. 5, pp. 374-376, 332016.

[24] M. A. T. Sanduleanu and E. Stikvoort, "Highly linear, varactor-less, 24GHz IQ oscillator," 2005 IEEE Radio Frequency integrated Circuits (RFIC) Symposium Digest of Papers, Long Beach, CA, USA, 2005, pp. 577-580.

[25] C. Gourdon, R. Sevin, D. Domnesque and M. Camiade, "A High Linearity, Low Phase Noise and High Operating Temperature Ku-band MMIC VCO-Prescaler, suitable for Complex Modulations ACC radar source," 2006 European Microwave Conference, Manchester, 2006, pp. 91-94.

[26] E. Ayranci, K. Christensen and P. Andreani, "Enhancement of VCO Linearity and Phase Noise by Implementing Frequency Locked Loop," EUROCON 2007 - The International Conference on "Computer as a Tool", Warsaw, 2007, pp. 2593-2599.

[27] W. Tan, G. Chen and H. Zhang, "A 1-GHz LC voltage-controlled oscillator with high linearity and wide range," 2008 IEEE International Conference on Electron Devices and Solid-State Circuits, Hong Kong, 2008, pp. 1-4. 
[28] K. Miyanaga, W. Lee, A. Kato, S. Hirano and K. Ishida, "VCO gain nonlinearity calibration with frequency offset/drift compensation for 3G/GSM/EDGE polar modulation transmitter," 2011 IEEE Radio and Wireless Symposium, Phoenix, AZ, 2011, pp. 231-234.

[29] B. Xi, Z. Zhanghua, S. Lihua, Z. Junhong and Y. Jie, "A Wide Tuning Range and High Linearity VCO Circuit Design," 2012 International Conference on Computer Science and Electronics Engineering, Hangzhou, 2012, pp. 208-211.

[30] T. He, Y. Jiang, Y. Du, S. Sin, S. U and R. P. Martins, "A 10MHz BW 78dB DR CT $\Sigma \Delta$ modulator with novel switched high linearity VCO-based quantizer," 2012 IEEE International Symposium on Circuits and Systems, Seoul, 2012, pp. 65-68.

[31] S. K. Saha, "Linear VCO with sine wave output," in IEEE Transactions on Instrumentation and Measurement, vol. IM-35, no. 2, pp. 152-155, June 1986.

[32] Youming Zhang, Fengyi Huang, Xusheng Tang and Mingchi Shao, "A broadband highly linear 3.5-6GHz CMOS VCO for multi-standard wireless transceivers," 2012 IEEE 14th International Conference on Communication Technology, Chengdu, 2012, pp. 380-384.

[33] Qiuting Huang, "Phase noise to carrier ratio in LC oscillators," in IEEE Transactions on Circuits and Systems I: Fundamental Theory and Applications, vol. 47, no. 7, pp. 965-980, Jul 2000.

[34] D. Murphy, J. J. Rael and A. A. Abidi, "Phase Noise in LC Oscillators: A Phasor-Based Analysis of a General Result and of Loaded Q," in IEEE Transactions on Circuits and Systems I: Regular Papers, vol. 57, no. 6, pp. 1187-1203, June 2010.

[35] C. Plett, Lecture Notes for ELEC 5503, Oscillator. Carleton University at Ottawa, Winter semester, 2016.

[36] A. Hussein, S. Vasadi, M. Soliman, and J. Paramesh, A 50-to-60 GHz $65 \mathrm{~nm}$ CMOS All-Digital Fractional-N PLL with 220 fs rms Jitter, University of Pennsylvania, 2017.

[37] S. Saad, M. Mhiri, A. B. Hammadi and K. Besbes, "A fine frequency tuning for LC-DCO using enhanced capacitive degeneration technique," 2017 International Conference on Engineering \& MIS (ICEMIS), Monastir, 2017, pp. 1-6.

[38] L. Fanori, A. Liscidini and R. Castello, "3.3GHz DCO with a frequency resolution of $150 \mathrm{~Hz}$ for All-digital PLL," 2010 IEEE International Solid-State Circuits Conference (ISSCC), San Francisco, CA, 2010, pp. 48-49.

[39] L. Fanori, A. Liscidini and R. Castello, "Capacitive Degeneration in LC-Tank Oscillator for DCO Fine-Frequency Tuning," in IEEE Journal of Solid-State Circuits, vol. 45, no. 12, pp. 2737-2745, Dec. 2010.

[40] N. Hosseini and H. Nabovati, "New geometry for improving Q-factor of spiral integrated inductor on low cost integrated circuit process," 2009 Canadian Conference on Electrical and Computer Engineering, St. John's, NL, 2009, pp. 1095-1098.

[41] S. Weisberg, Applied Linear Regression. Minneapolis, MN, USA: Willey, 2014, pp. 1-36. 
[42] A. I. Hussein, S. Saberi and J. Paramesh, "A $10 \mathrm{~mW}$ 60GHz 65nm CMOS DCO with $24 \%$ tuning range and $40 \mathrm{kHz}$ frequency granularity," 2015 IEEE Custom Integrated Circuits Conference (CICC), San Jose, CA, 2015, pp. 1-4.

[43] Y. Wang, Y. Liu, A. Agrawal and A. Natarajan, "A 74.6GHz-83.6GHz digitally controlled oscillator with $370 \mathrm{kHz}$ frequency resolution in 65nm CMOS," 2016 IEEE Radio and Wireless Symposium (RWS), Austin, TX, 2016, pp. 176-178.

[44] J. Bai, J. Lee, J. Zhang and N. Rohani, "A 28-nm CMOS 40-GHz high-resolution digitally controlled oscillator for automotive radar applications," 2017 IEEE 17th Topical Meeting on Silicon Monolithic Integrated Circuits in RF Systems (SiRF), Phoenix, AZ, 2017, pp. 91-93.

[45] EMX, 3D EM Simulator. Computer Software. Only available at Microsemi (Microchip) Corporation. Integrand Software, Inc. LINUX-PC, 2016.

[46] Calibre xL, Circuit Verification With Calibre. Computer Software. Only available at Microsemi (Microchip) Corporation. Mentor Graphics, A Siemens Business. LINUX-PC, 2018.

[47] J. Rogers, F. Dai, and C. Plett, Integrated Circuit Design for High-Speed Frequency Synthesis. Boston: Artech House, 2006. 


\section{APPENDIX A: SCHEMATIC DIAGRAMS}

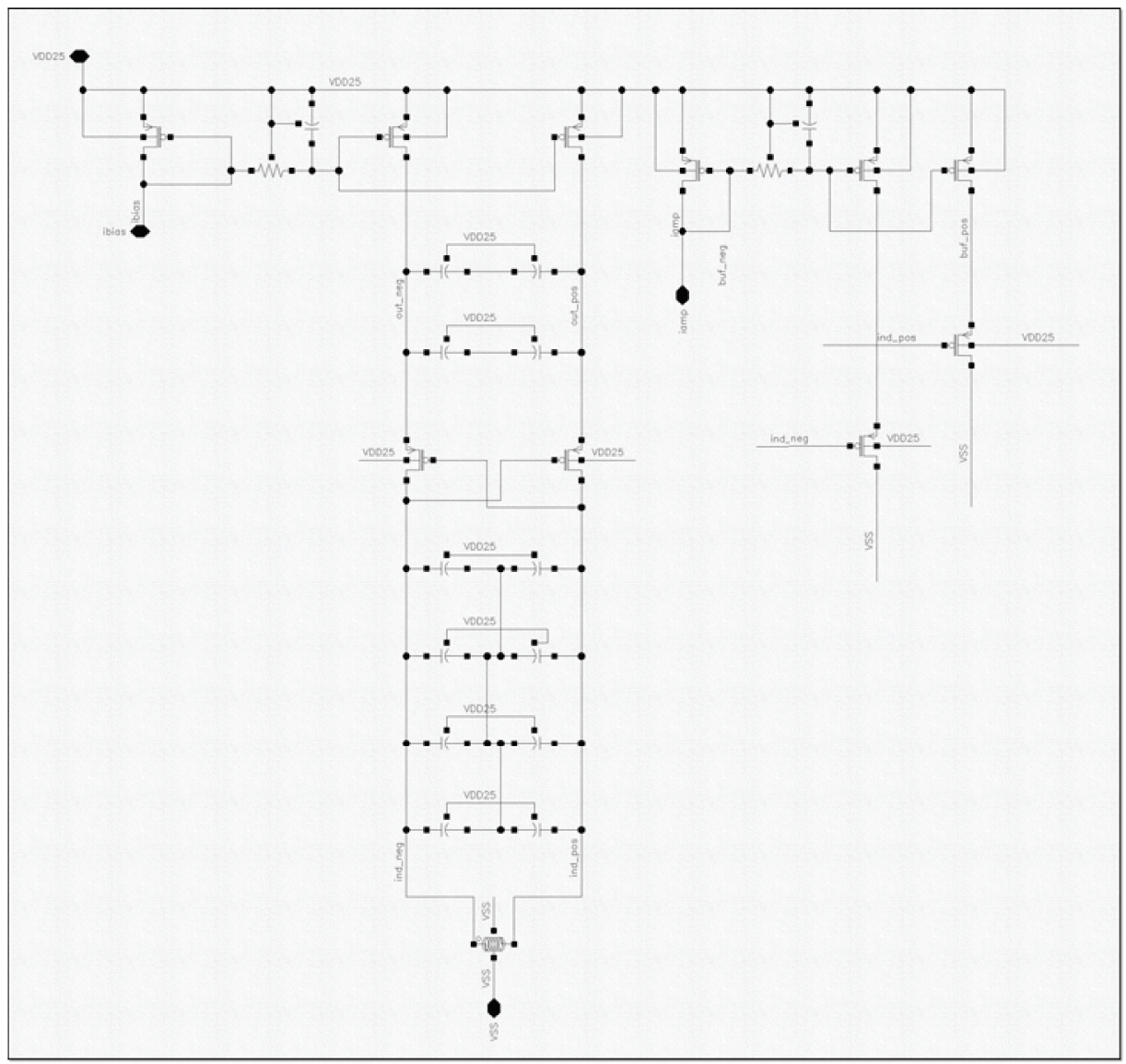

Figure 164: Oscillator core implementation in Cadence Virtuoso Schematic.

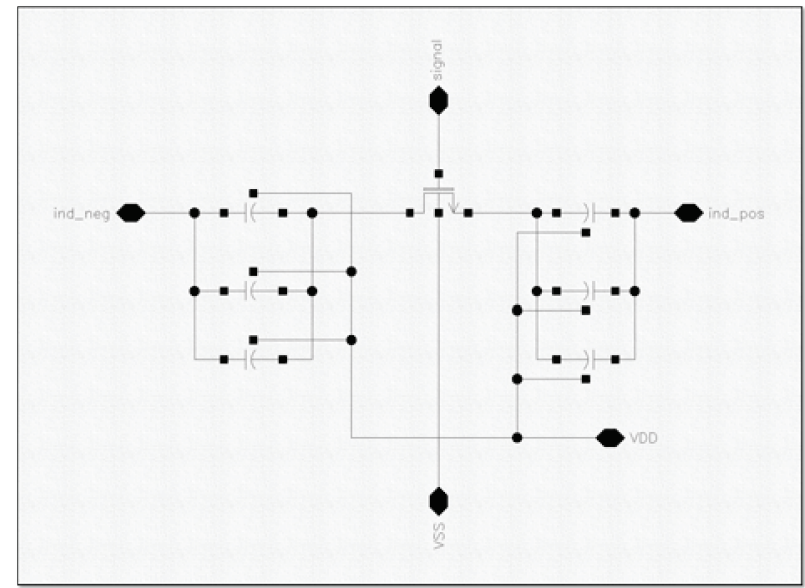

Figure 165: Bank schematic in Cadence Virtuoso Schematic. 


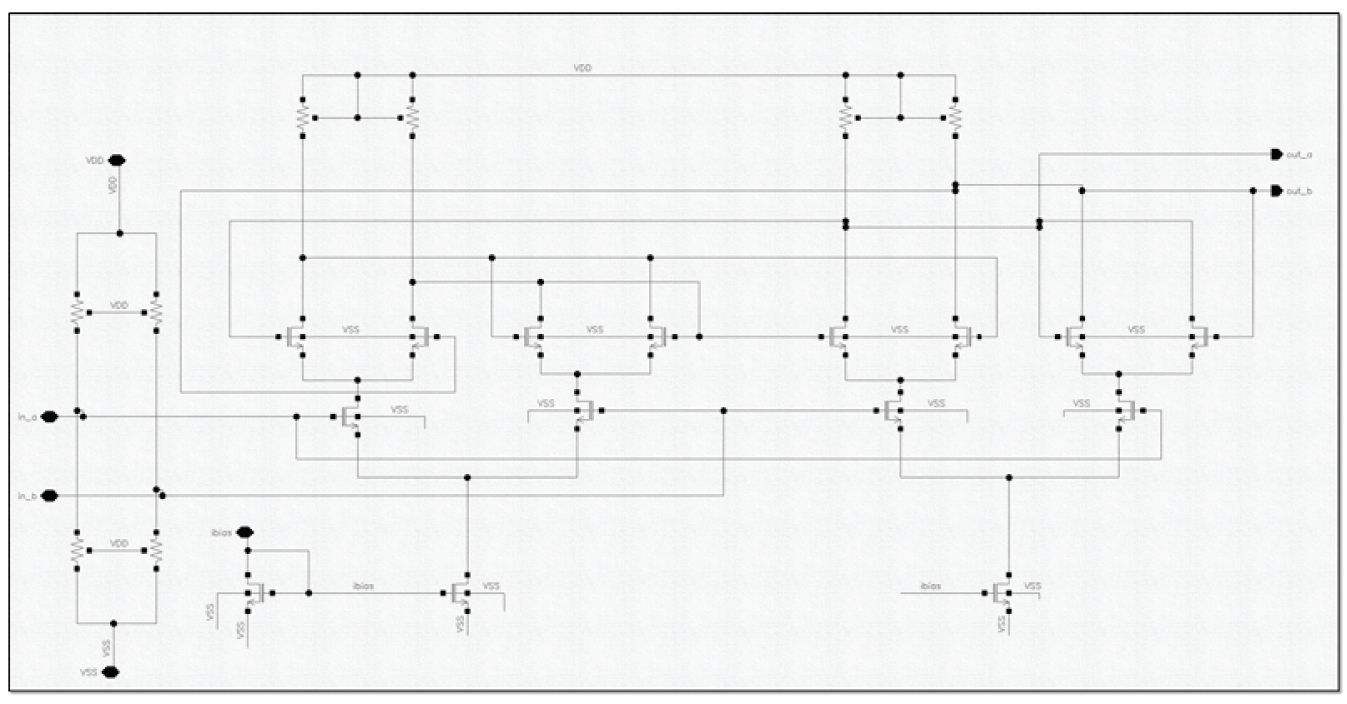

Figure 166: CML schematic in Cadence Virtuoso Schematic.

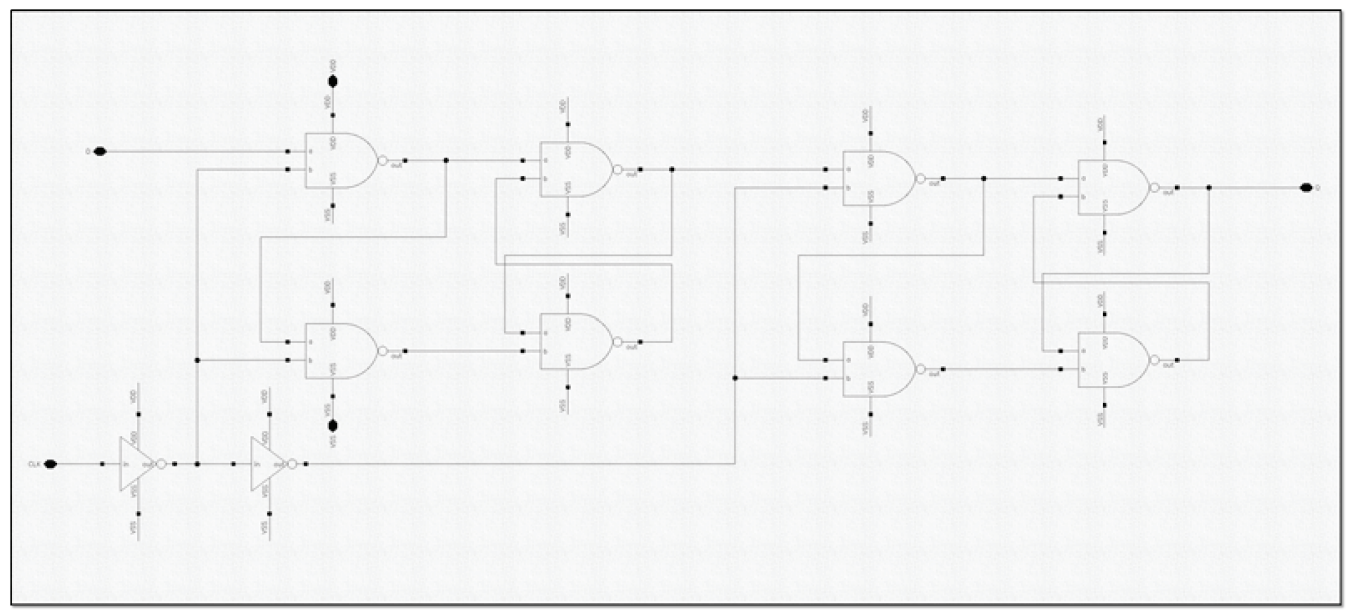

Figure 167: Flip-flop schematic in Cadence Virtuoso Schematic. 


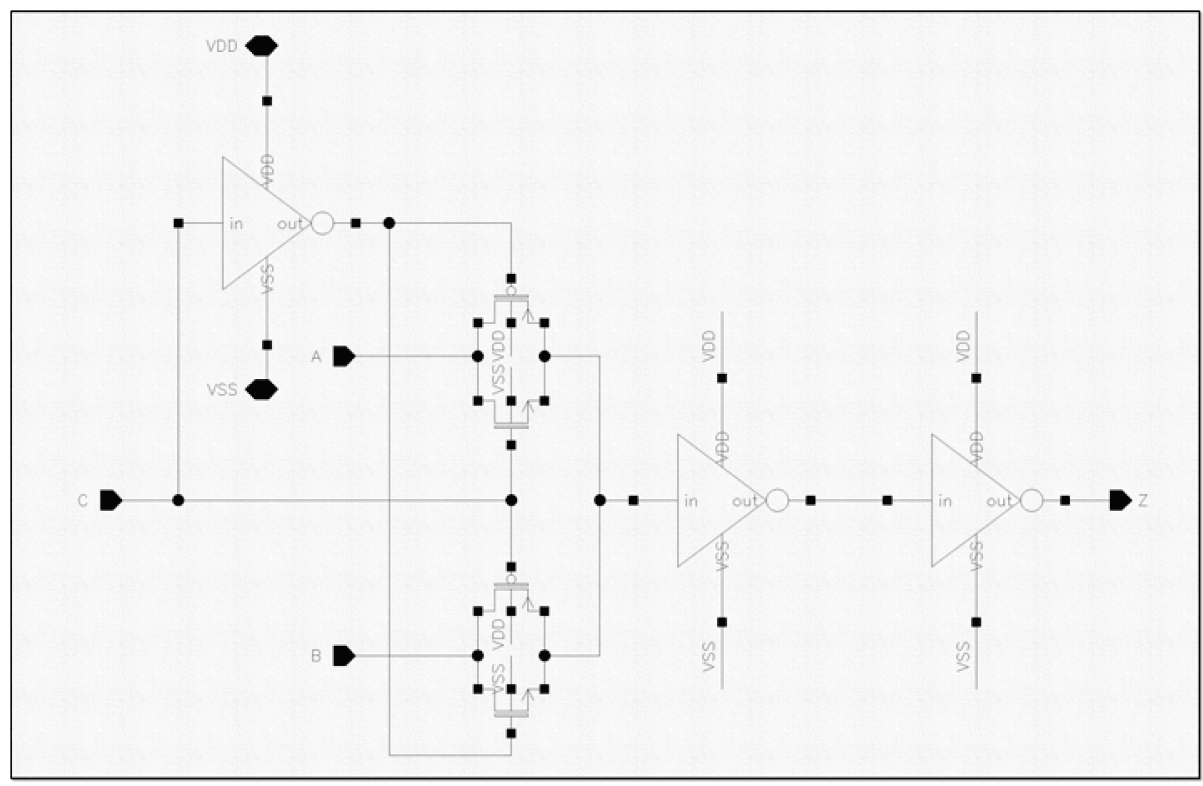

Figure 168: Mux schematic in Cadence Virtuoso Schematic.

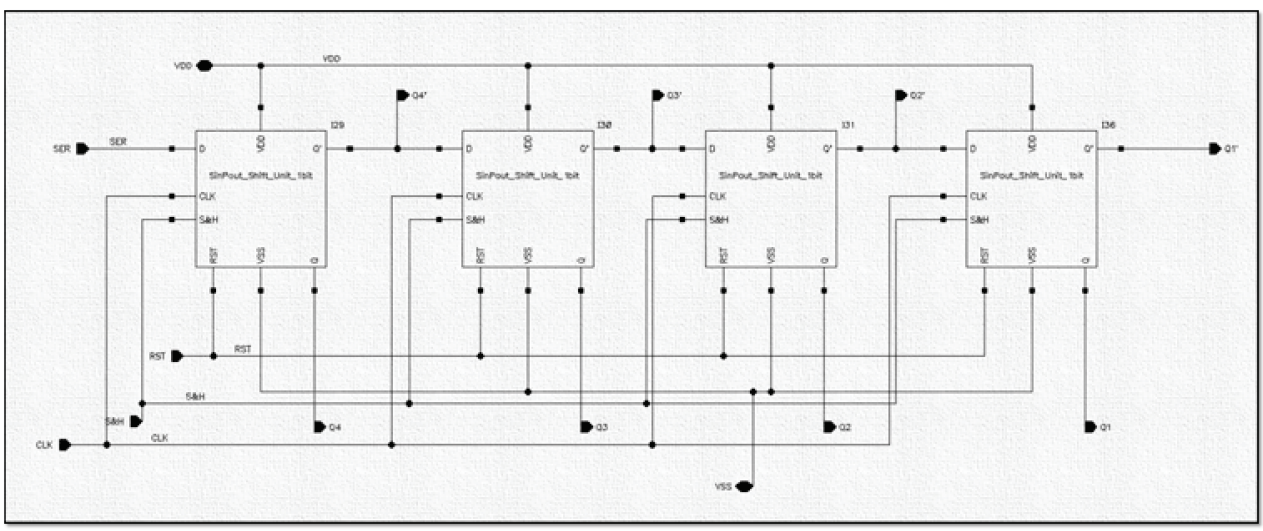

Figure 169: A 4-bit Shift register schematic in Cadence Virtuoso Schematic. 


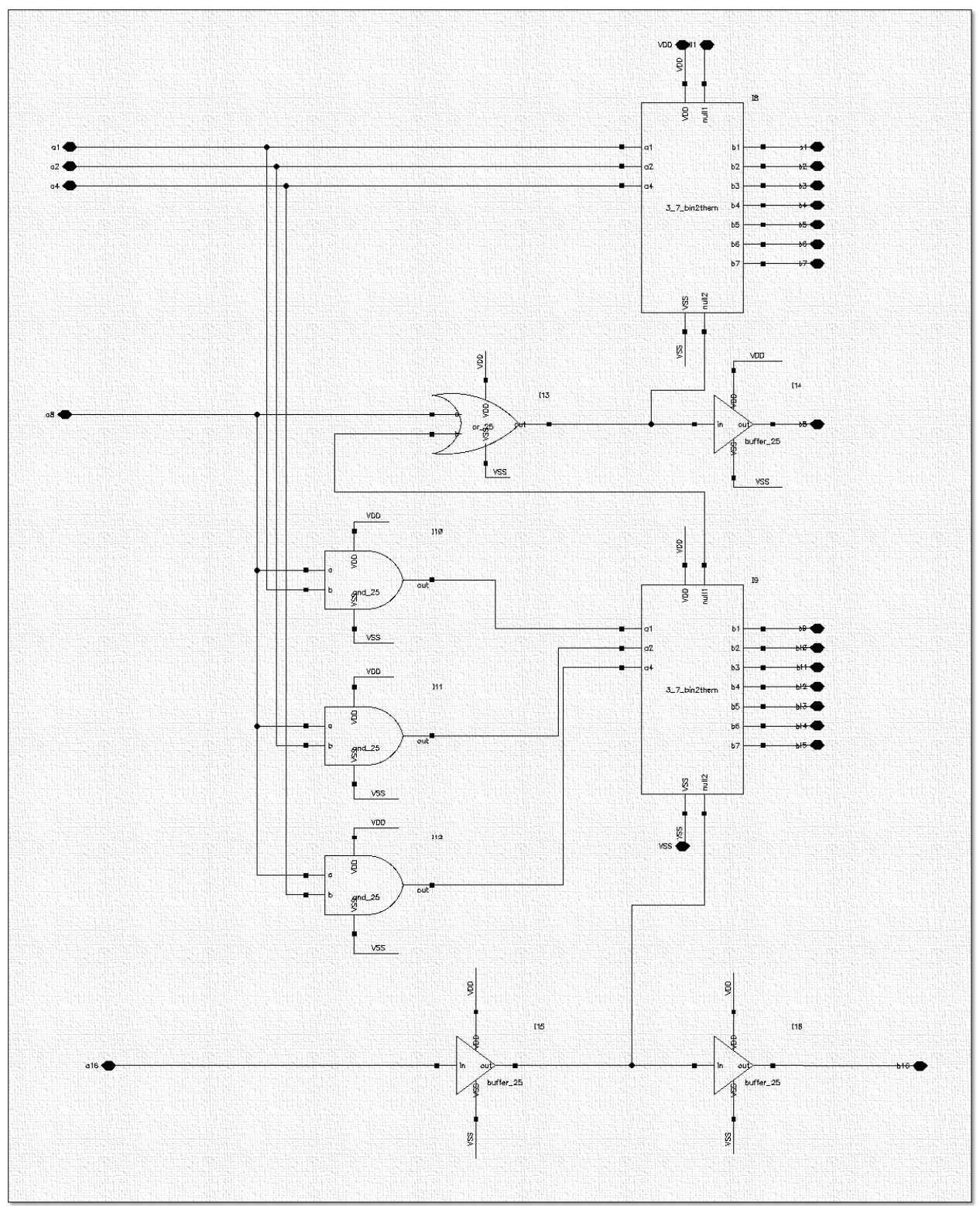

Figure 170: Thermometer to binary 5-16 bit decoder schematic in Cadence Virtuoso Schematic. 


\section{APPENDIX B: BONDING AND PACKAGING}

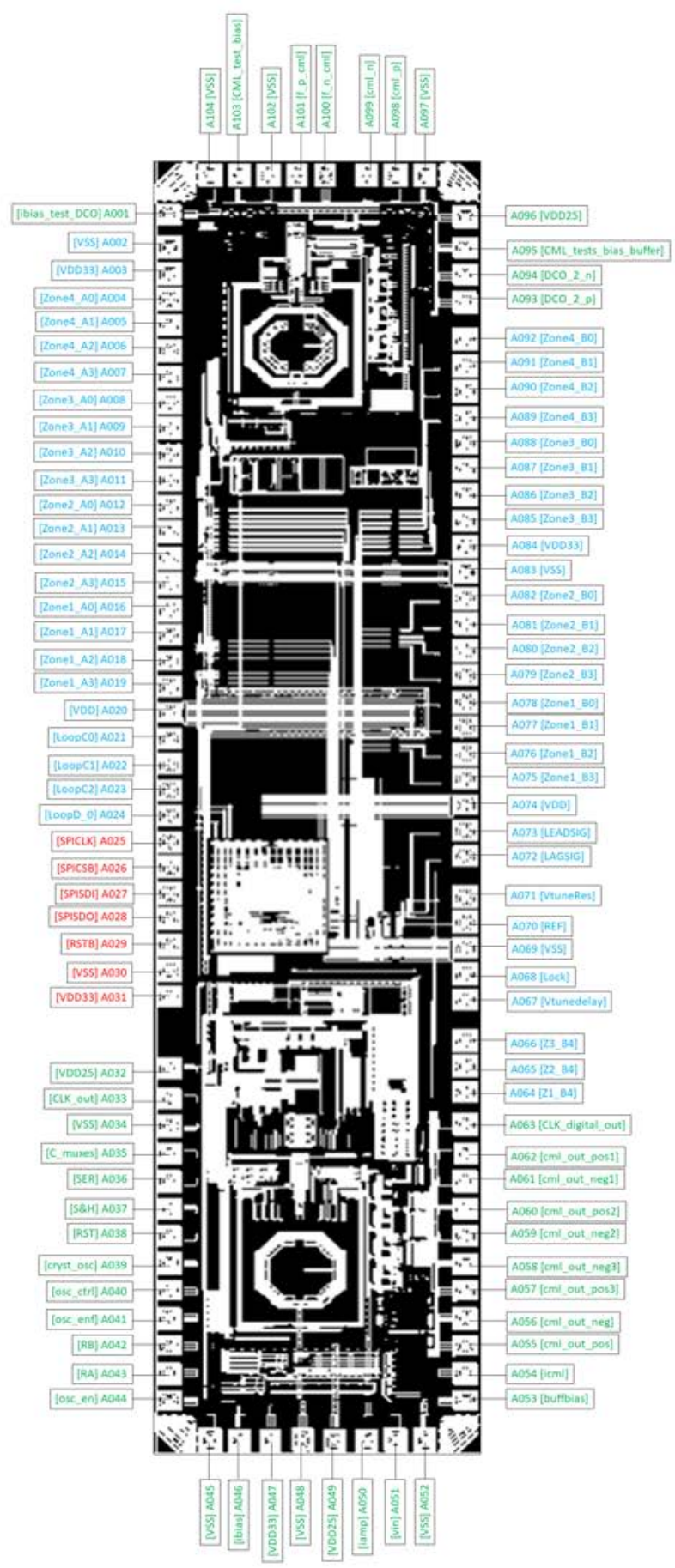

Figure 171: Pin layout of ADPLL chip. 


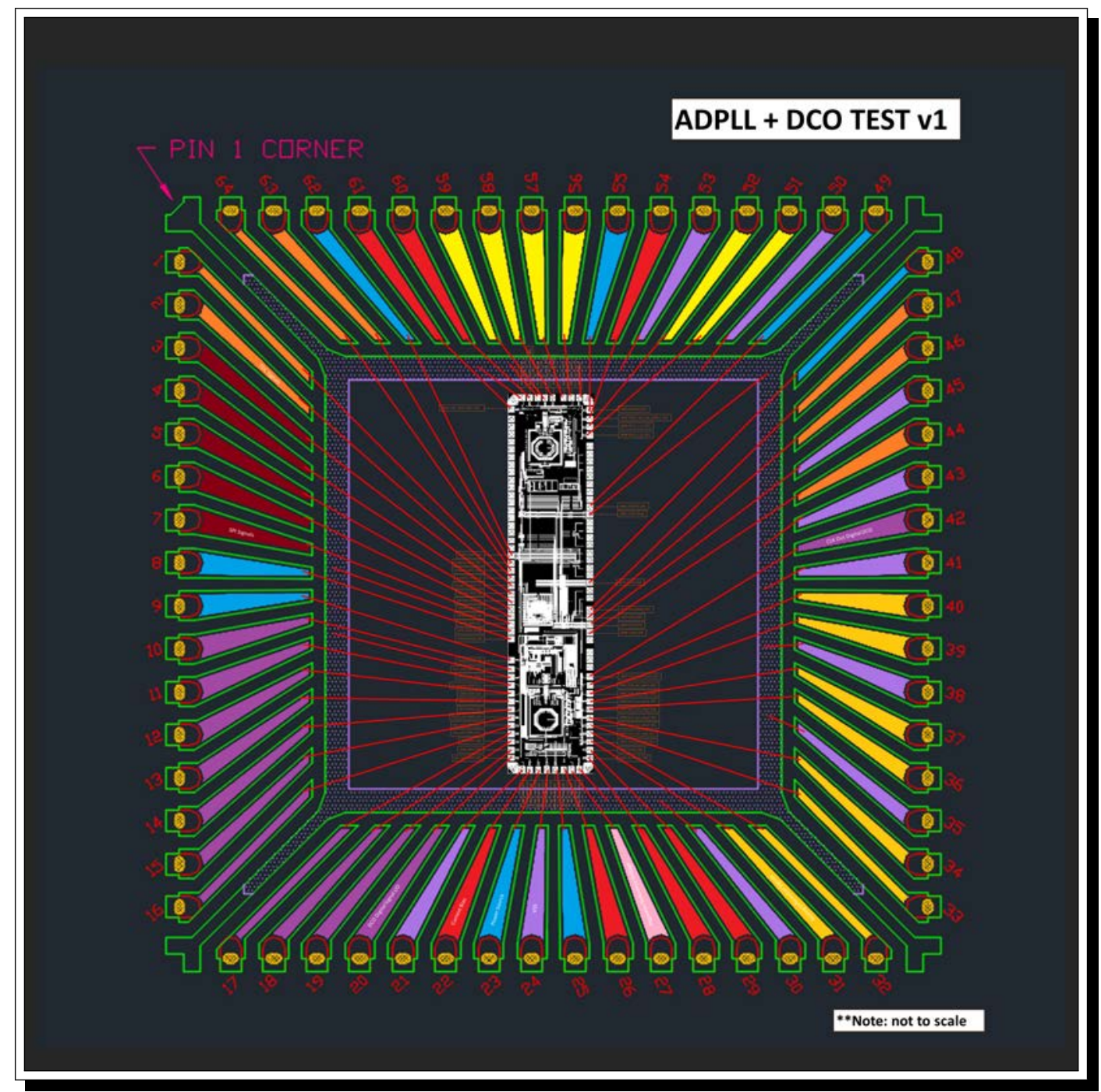

Figure 172: Conceptual bonding diagram of DCO chip. QFN64 packaging was used. 


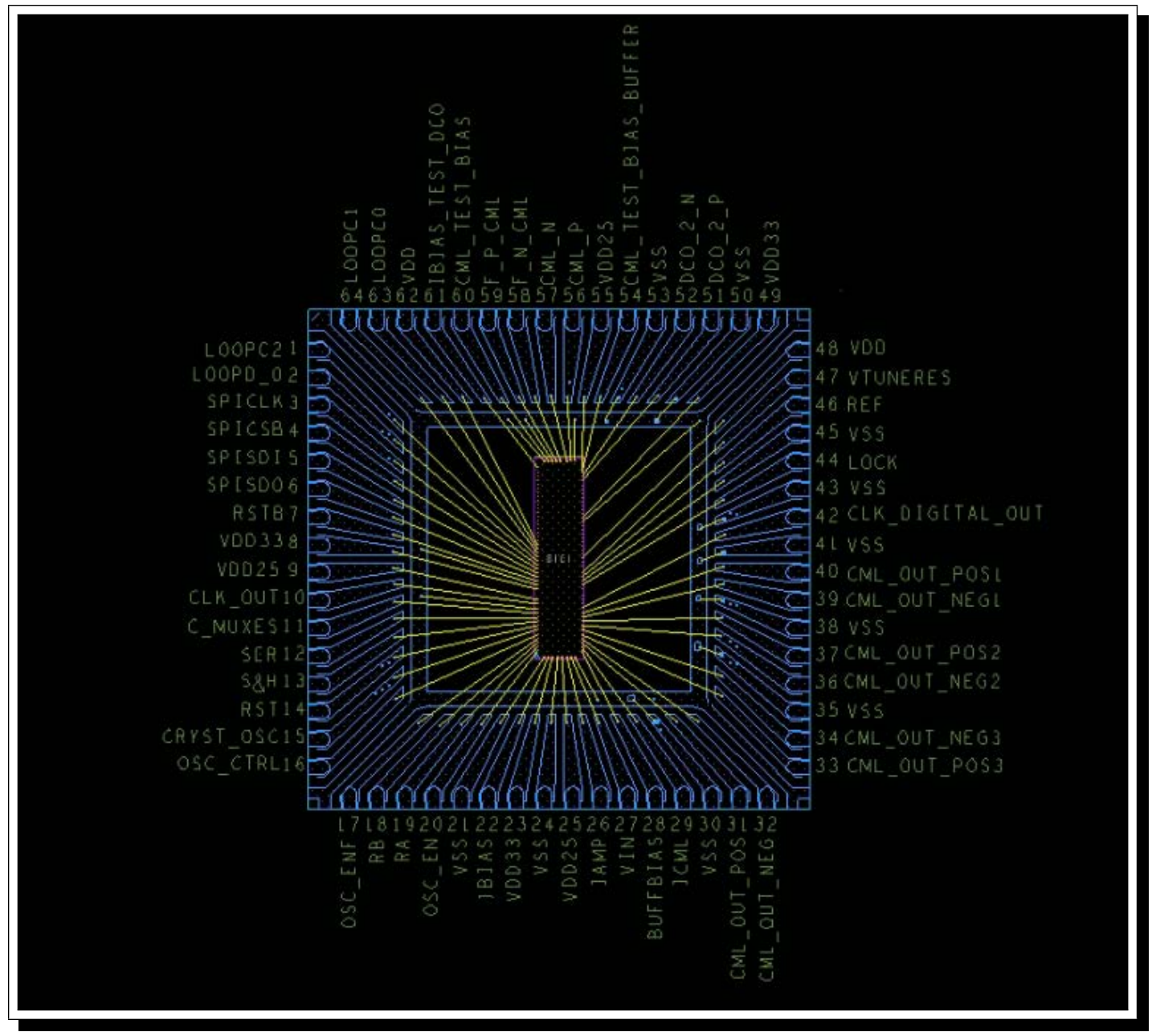

Figure 173: Final bonding diagram. 


\section{APPENDIX C: TEST PCBs}

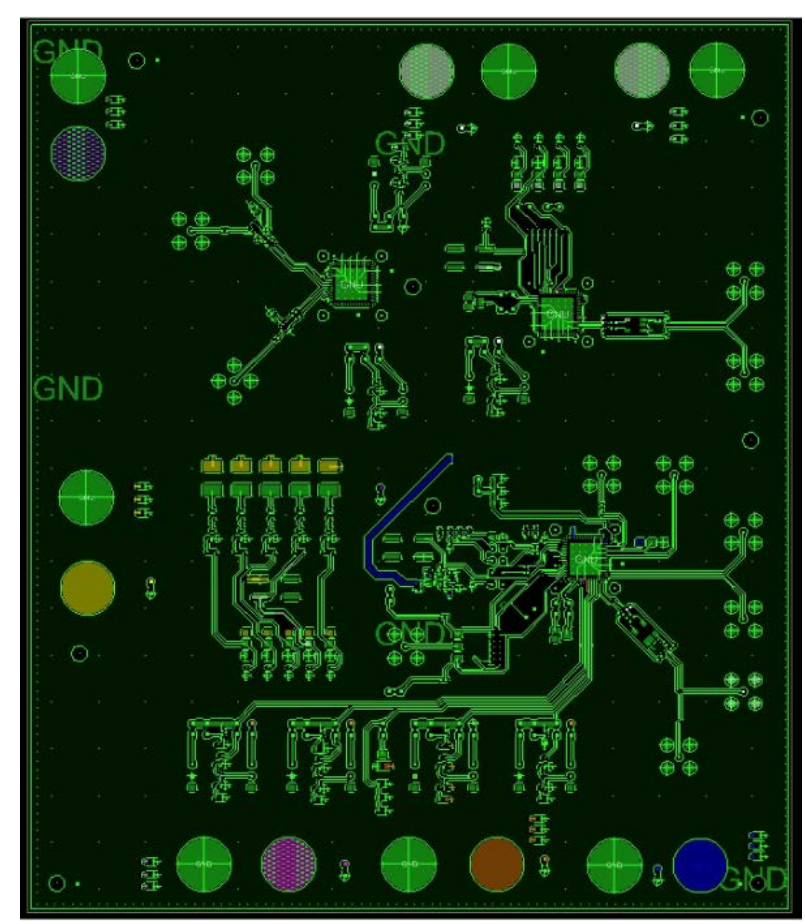

Figure 174: Layer 1 of main PCB.

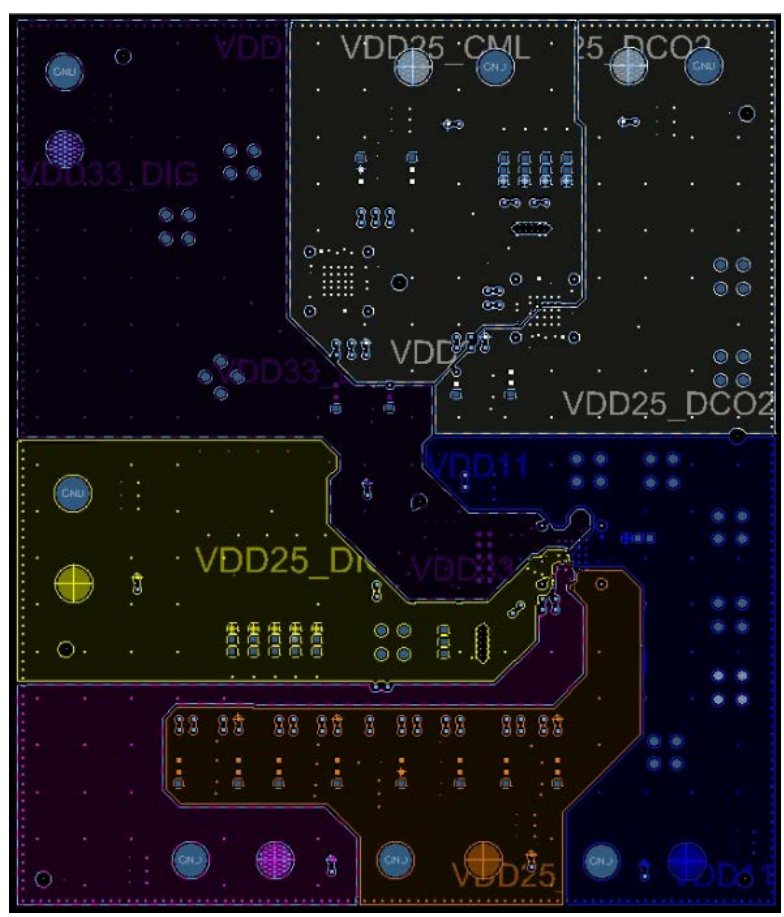

Figure 175: Layer 2 of main PCB. 


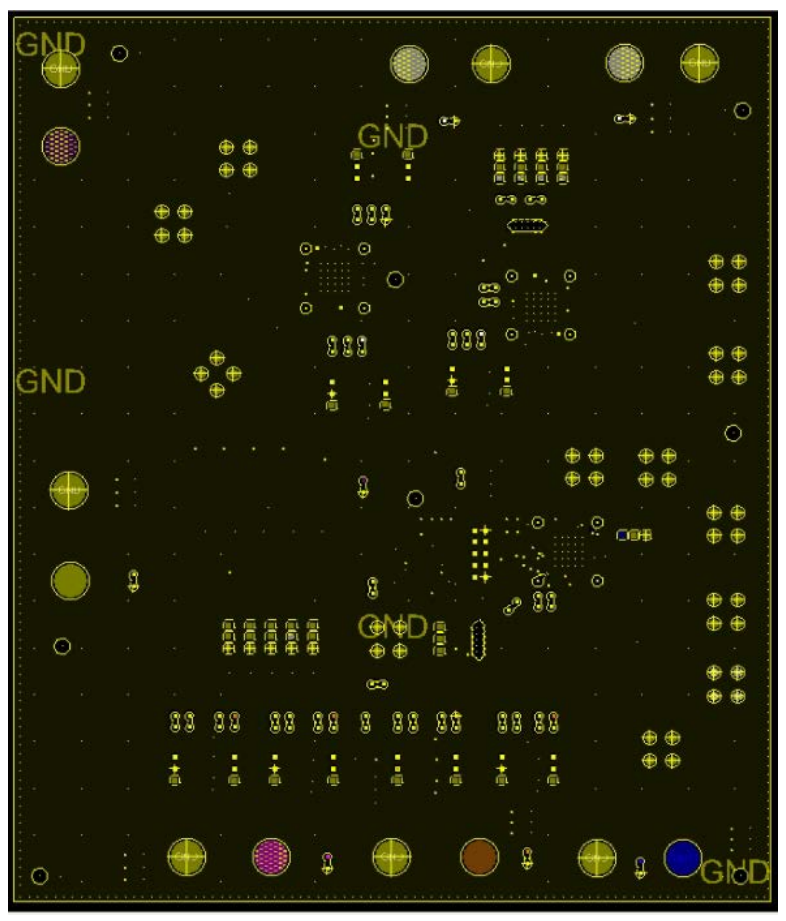

Figure 176: Layer 3 of main PCB.

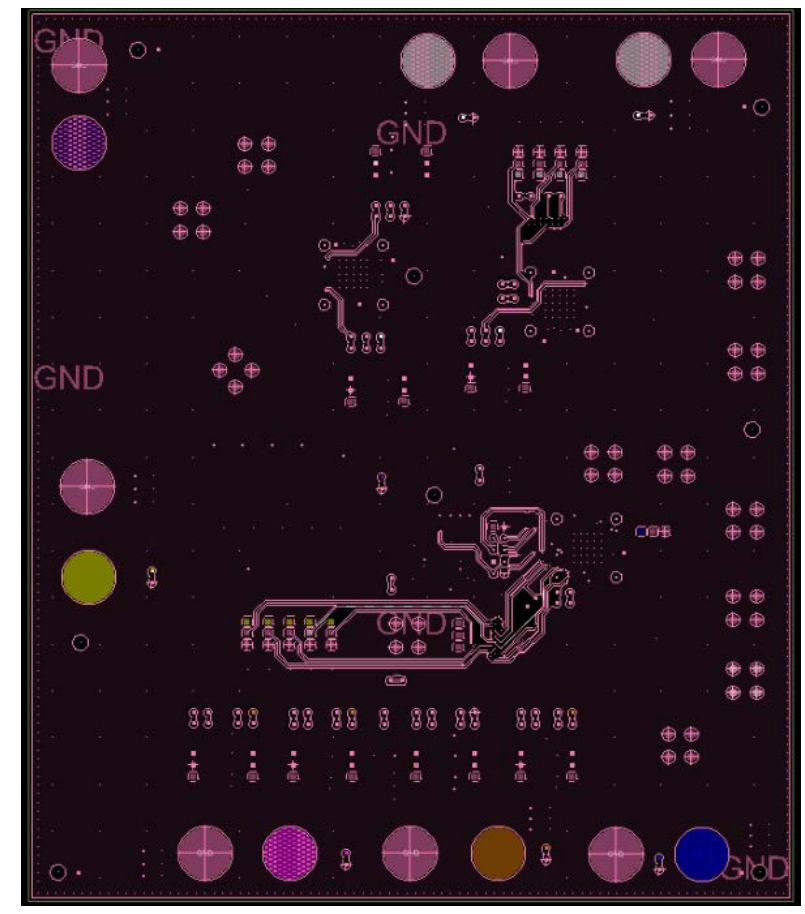

Figure 177: Layer 4 of main PCB. 


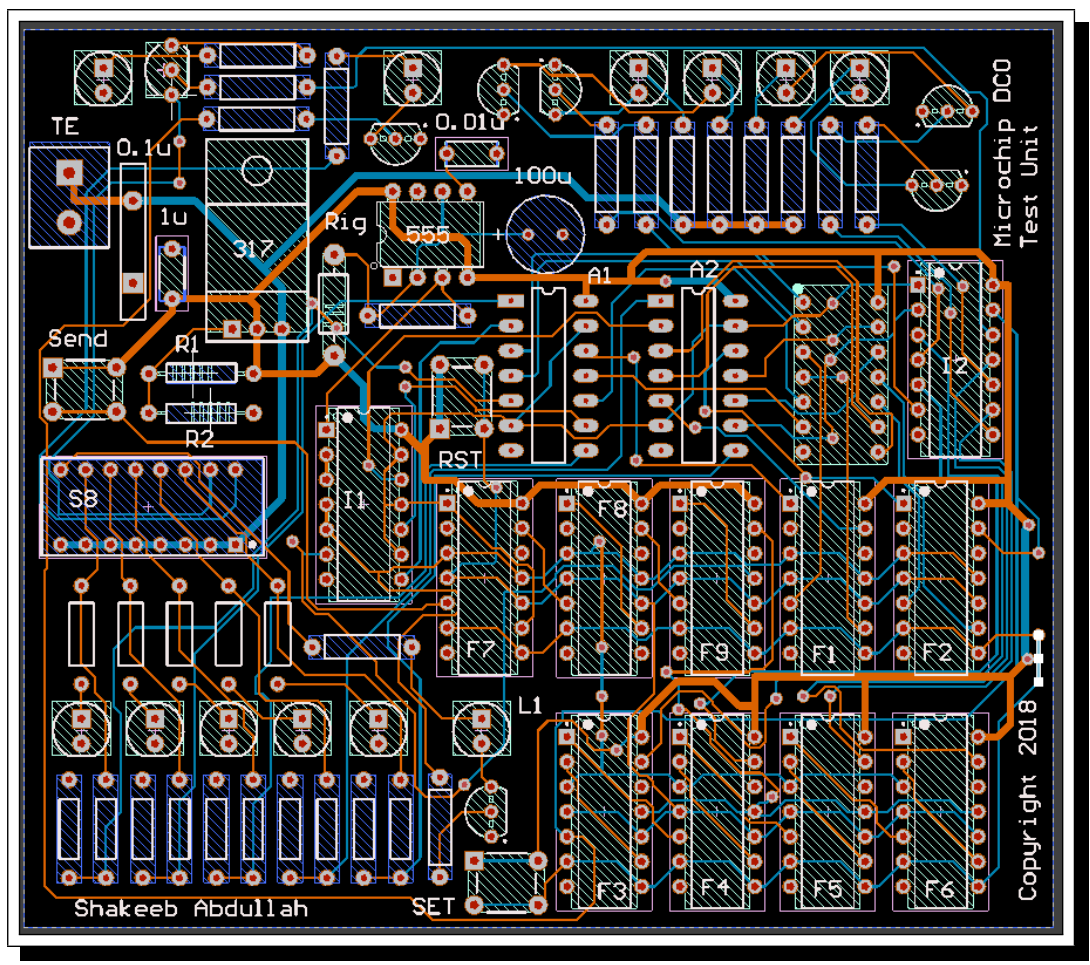

Figure 178: PCB layout of the 4-bit serial to parallel interface.

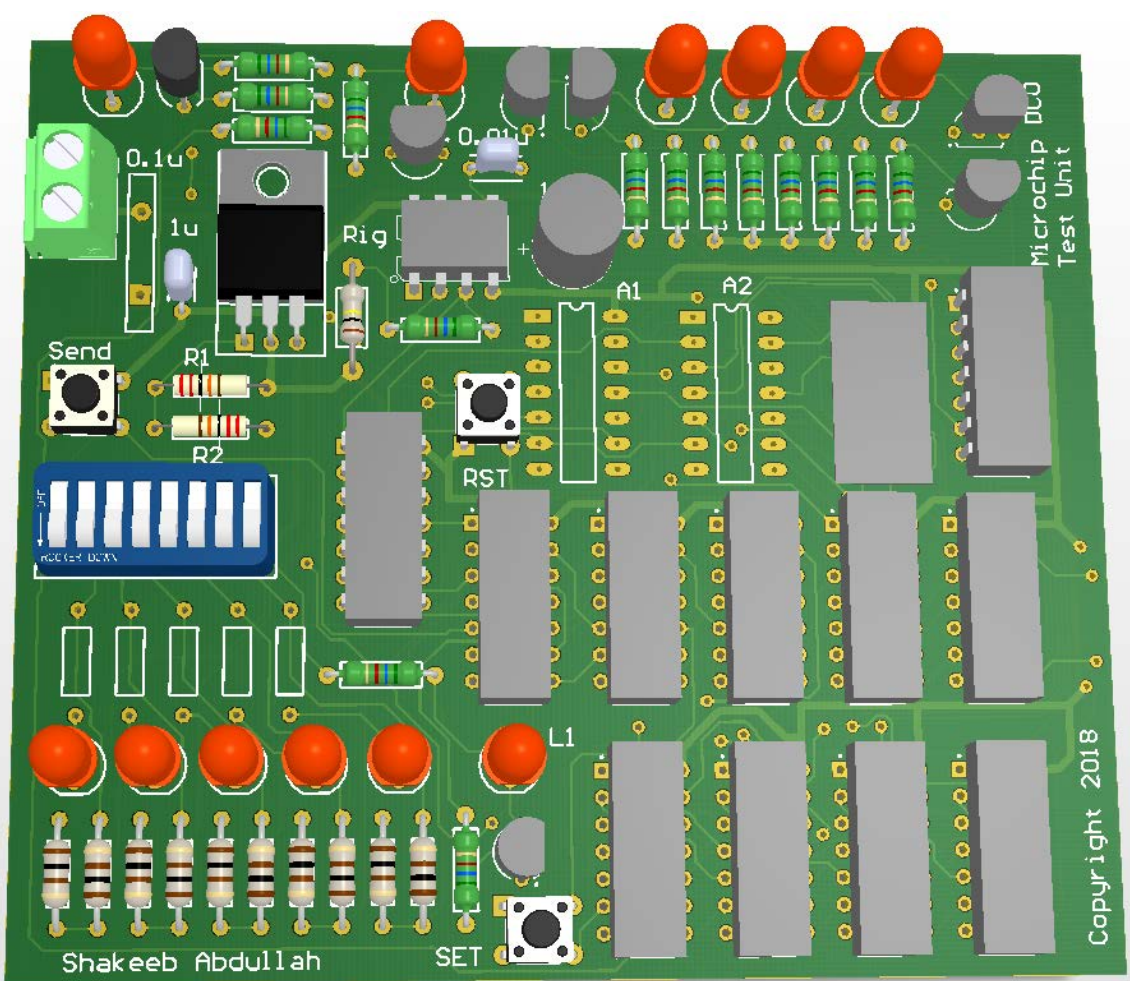

Figure 179: A 3D rendering of the 4-bit serial to parallel interface PCB. 


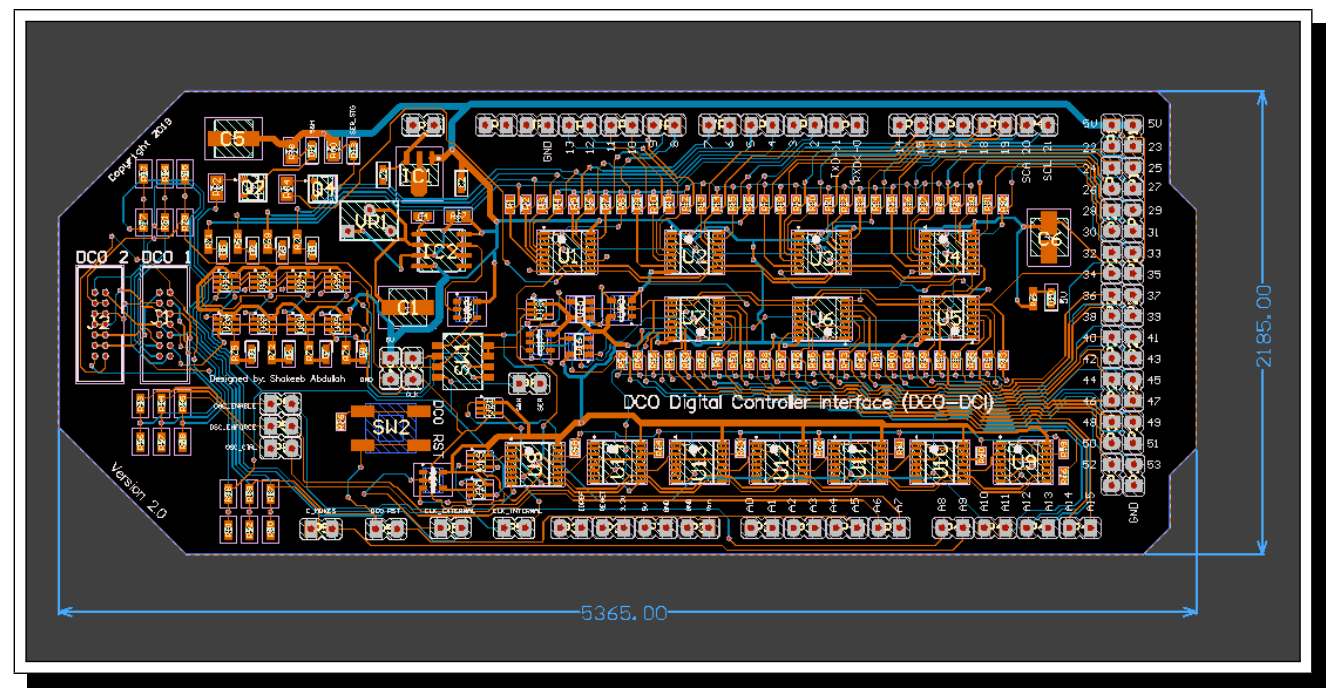

Figure 180: Main DCI PCB used to control DCO 1.

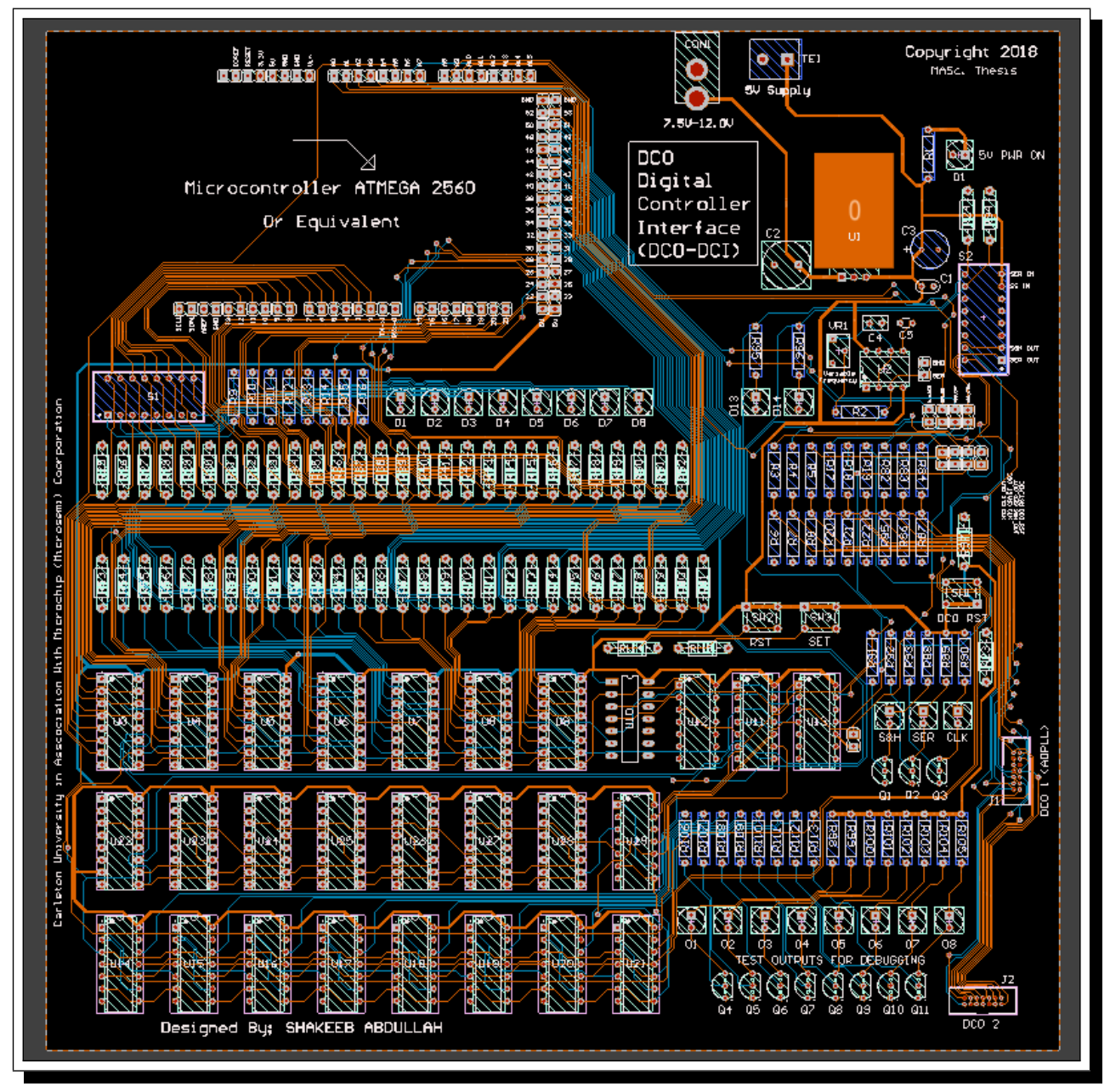

Figure 181: Modified DCI PCB. 


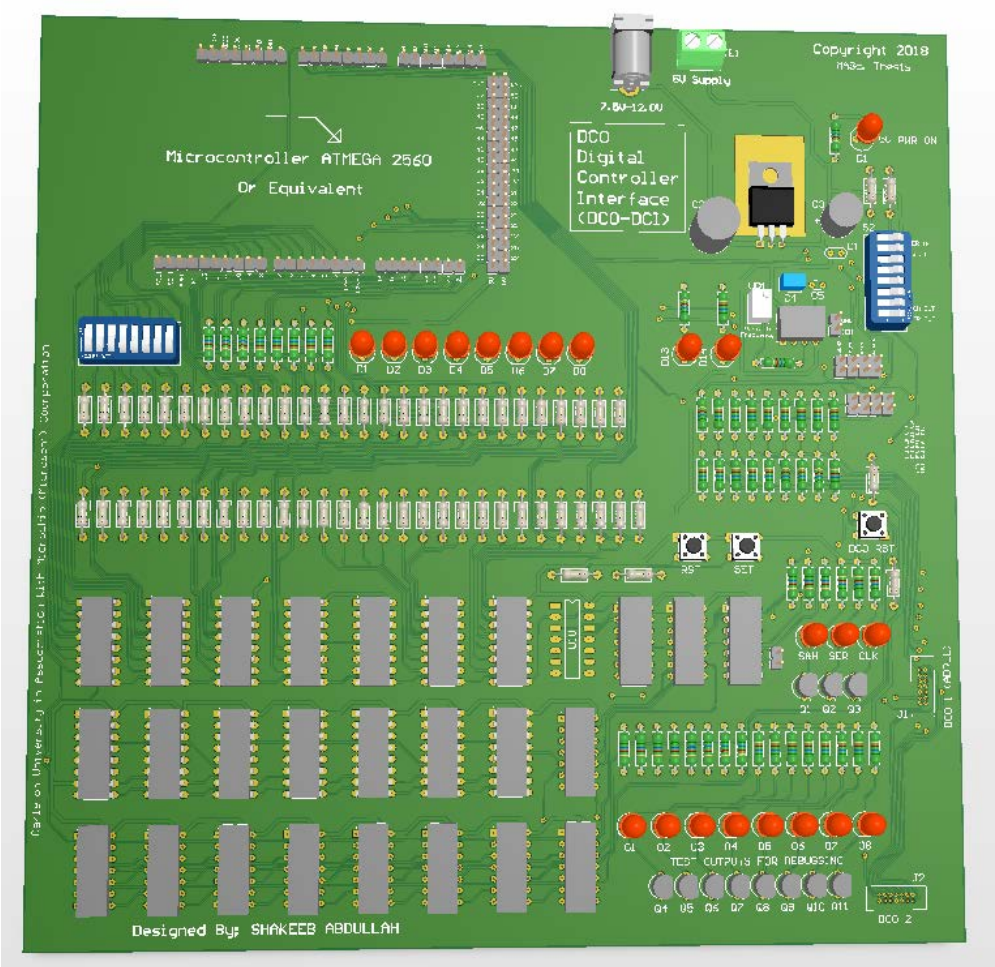

Figure 182: A 3D rendering of the DCI 2 PCB.

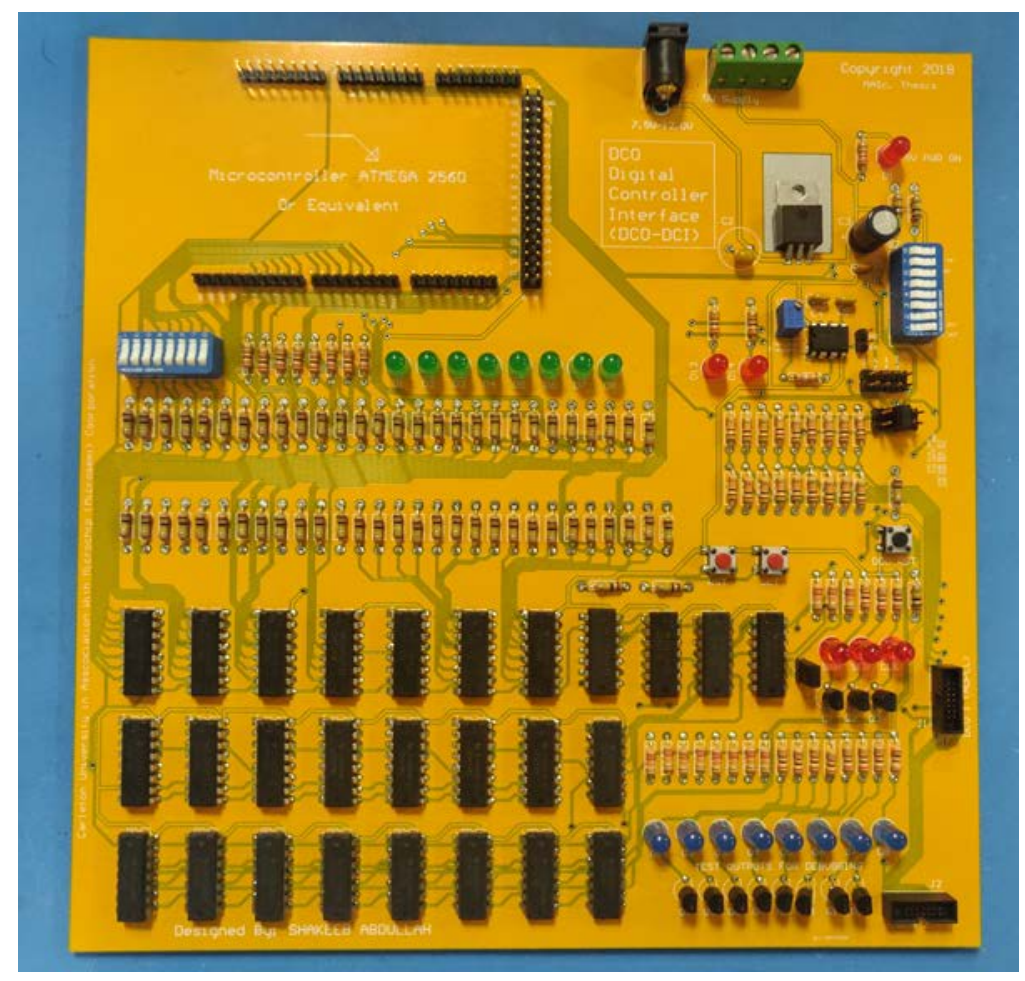

Figure 183: Fabricated DCI 2 PCB. 


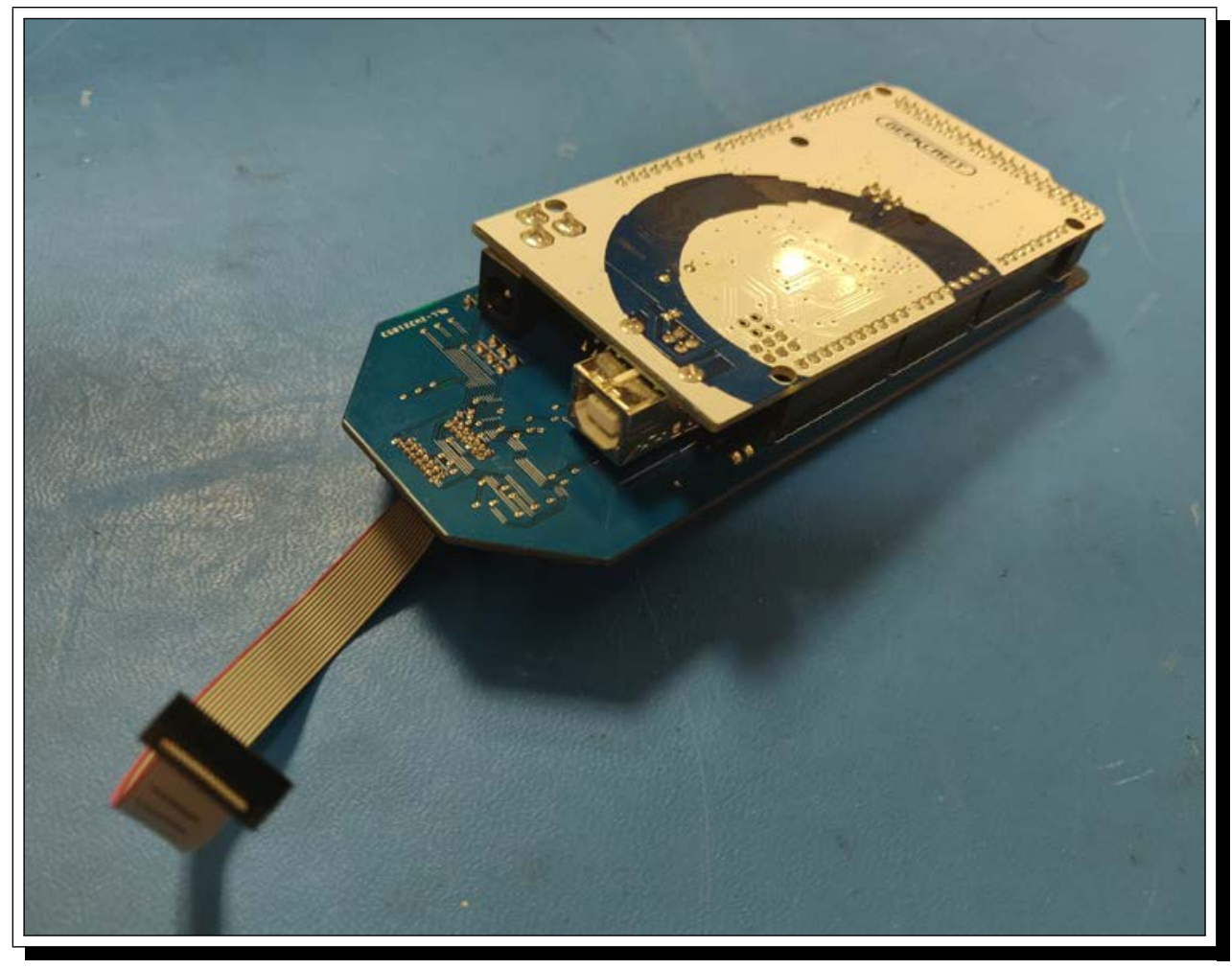

Figure 184: Example of how the DCI PCB interfaces with a MEGA 256. The micro-controller (pins) fits on the bottom of the board. 


\section{APPENDIX D: SOFTWARE TEST GUI}

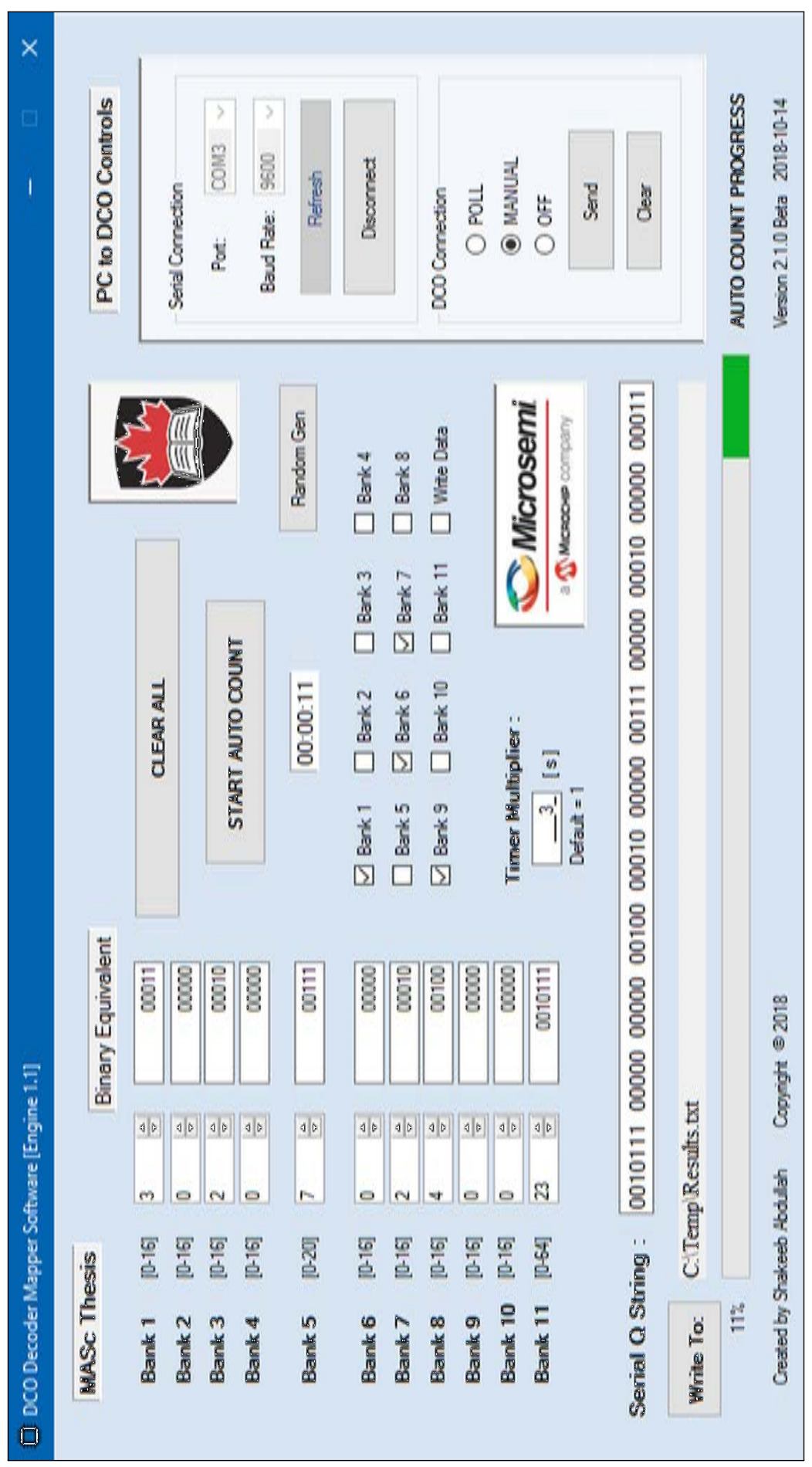

Figure 185: Software GUI used to control the DCO via DCI PCB. 


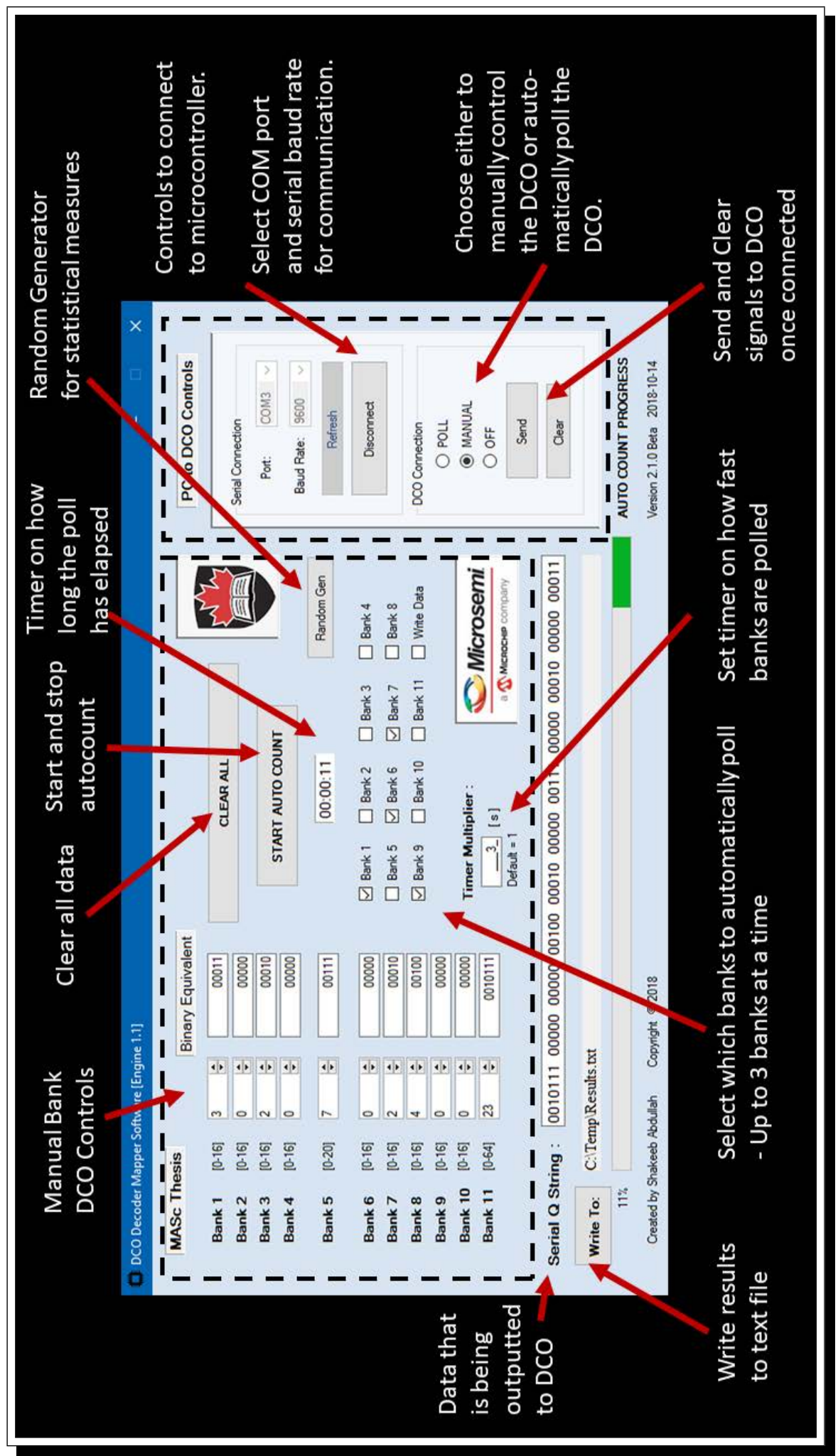

Figure 186: The functions of the software (GUI) used to control DCO. 